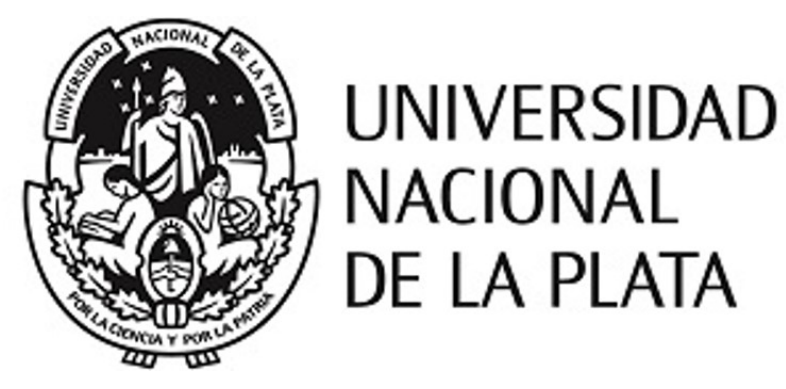

FACULTAD DE INGENIERÍA

DEPARTAMENTO DE ELECTROTECNIA

TESIS DOCTORAL:

\title{
MODELADO Y SIMULACIÓN DE TECNOLOGÍAS PARA EL TRATAMIENTO DE LA DIABETES
}

Tesis escrita por Nicolás Rosales para el título de Doctor en Ingeniería de la Universidad Nacional de La Plata

Directores de tesis:

Fabricio Garelli

Hernán De Battista

Miembros del jurado:

Dr. Alejandro Hernán González

Dr. Marcelo Raúl Risk

Dr. Gastón Schlottauer

La Plata, Argentina

Marzo de 2020 

Dedicado a todxs lxs que me apoyaron y confiaron en mi, compartieron mi risa y lágrimas... 



\section{Agradecimientos}

Recorro en mi mente el camino que me trajo hasta este punto, una página donde agradecer a quienes estuvieron ahí para apoyarme y motivarme, conscientemente o no. Mis pensamientos se tiñen de nostalgia y los recuerdos bailotean en mi imaginación. Por eso, mis gracias totales

A mi compañera Johanna, quien estuvo junto a mi en este camino.

A mi familia Alejandro, Silvia, Nadia, Jere, Emi y Paula.

A mi otra familia, de la vida, esa telaraña de relaciones de amigxs y hermanxs. A todxs ustedes.

A mis queridos directores Faber y Hernán por su apoyo todos estos años. Por tolerar mis idas y vueltas, agarres y locuras. Y a mi gran grupo de trabajo Diabeteam: Emi, Ceci, Delfi y Lean. Al GCA por la bienvenida al grupo, el apoyo y los grandes asados. A la gente del LEICI por los buenos momentos, mates a granel, facturas, picadas de festejo y recuerditos de viajes. En especial a lxs becarixs que nos acompañamos y motivamos para seguir adelante.

A la música y con quienes juego con ella. Mi familia de Purparlé y a la eterna Perafernalia de Charlie Brownie. A quienes me dejaron compartir melodías y armonías que curan las heridas, sanan y acarician.

A Marcela, Patricio y Ricardo por las grandes experiencias, que sin su trabajo no hubiera podido alcanzar.

A Fabian y Maira por recibirme tan cálidamente en Bogotá y su hogar. Por llevarme por las rutas colombianas y el hermoso viaje compartido.

A Pep por su trabajo y perseverancia. Y a todo el grupo de Girona por aguantarme los mates charletas. En especial a Iván, Nerea y Mariela por dejarme hacer de su casa un hogar.

A la gente del SENYT por la buena onda y predisposición a aclarar mis dudas estocásticas.

A la Universidad publica y gratuita y a los organismos de investigación CONICET, CIC, Agencia. Y la comunidad científica argentina por los Congresos, el esfuerzo y la simpatía.

A SciHub, Stackexchange, Stackoverflow y a todxs lxs foristas que brindan sus conocimientos y soluciones. 



\section{Abstract}

En las últimas dos décadas hubo un gran crecimiento en la investigación y el desarrollo de sistemas de Páncreas Artificial (PA). Estos sistemas, compuestos por un lazo de control realimentado, una bomba de insulina y un sensor continuo de glucosa, permiten la regulación automática de glucosa para personas con Diabetes Mellitus Tipo I (DMT1). Aún así, las limitaciones que presenta este desafío, como los son la gran variabilidad inter- e intrapaciente, la incertidumbre y retardos del sistema, dificultan la concepción de un control de lazo cerrado puro. Además de estas limitaciones, el riesgo de exponer a la persona a hiper- e hipoglucemias es un gran impedimento a la hora de desarrollar las estrategias de control glucémico.

En esta tesis se plantean objetivos que cubren distintos aspectos del desarrollo de terapias para el control de la glucosa en la DMT1. En primer lugar, se desarrolló una plataforma de simulación que cuenta con un amplio espectro de configuraciones. Luego, a partir de la estimación de la insulina activa en el cuerpo (IOB), se proponen dos algoritmos para el tratamiento a lazo abierto: uno para períodos posprandiales y otro para la actividad física. A su vez, a partir de la limitación de IOB, se proponen técnicas de lazo cerrado. Por un lado, se propone un algoritmo que permite la transición en configuraciones híbridas, entre el bolo posprandial de lazo abierto y el controlador de lazo cerrado. Por otro lado, a partir de una participación interdisciplinaria, se presenta el desarrollo y ensayo clínico de un algoritmo de control de lazo cerrado sin bolo para la compensación de comidas. Por último, se presenta el algoritmo GSAFE para lazos cerrados de control puro donde se propone adaptar la salida de un controlador principal con el fin de imponer una limitación en la pendiente de caída de glucosa con el objetivo de prevenir futuras hipoglucemias debido a la sobreactuación. 



\section{Índice general}

\begin{tabular}{lll}
\hline Agradecimientos & V
\end{tabular}

\begin{tabular}{ll}
\hline Lista de figuras & XI
\end{tabular}

\begin{tabular}{lll}
\hline Lista de tablas & XV
\end{tabular}

\begin{tabular}{ll}
\hline Abreviaciones & XVII
\end{tabular}

\begin{tabular}{ll}
\hline Prefacio & XXI
\end{tabular}

$\begin{array}{ll}\text { 1. Introducción } & 3\end{array}$

1.1. Diabetes Mellitus . . . . . . . . . . . . . . . . . . 3

1.2. La vida con Diabetes . . . . . . . . . . . . . . . . . . . 5

1.2.1. Complicaciones . . . . . . . . . . . . . . . . . 7

1.2 .2$. Tratamientos . . . . . . . . . . . . . . . . 8

1.2.3. El rol de la tecnología . . . . . . . . . . . . . . . . . . 12

1.3. Páncreas Artificial . . . . . . . . . . . . . . . . . . . . . . . 17

1.3.1. Diseño de controladores . . . . . . . . . . . . . . . . . . . 18

1.3.2. Desafíos del control de glucosa . . . . . . . . . . . . . . . 20

1.3.3. Ensayos clínicos . . . . . . . . . . . . . . . . . . . . . 21

1.3.4. Control bihormonal . . . . . . . . . . . . . . . . . 22

1.4. Conclusiones . . . . . . . . . . . . . . . . . . . 22

\begin{tabular}{ll}
\hline 2. Modelos y simulación in-silico & 23
\end{tabular}

2.1. Reseña de modelos . . . . . . . . . . . . . . . . . . . . . . . . . . 24

2.1.1. Modelo Sorensen . . . . . . . . . . . . . . . . . . 24

$2.1 .2 . \quad$ Modelo Bergman . . . . . . . . . . . . . . . . . . . 28

$2.1 .3 . \quad$ Modelo Cambridge . . . . . . . . . . . . . . . . . . . . . . 29

2.1.4. Modelo UVA/Padova . . . . . . . . . . . . . . . . . . . . . 32

2.1.5. Comparación de modelos . . . . . . . . . . . . . . . . . . . . . 39

2.2. Plataforma de simulación UNLP . . . . . . . . . . . . . . . . . . . 41

2.2.1. Bloques . . . . . . . . . . . . . . . . . . 42

2.2.2. Variación intra-paciente . . . . . . . . . . . . . . . 46

2.2.3. Absorción de carbohidratos y comidas mixtas . . . . . . . . . . . . . 49

2.2.4. Comentarios finales . . . . . . . . . . . . . . . . 50

2.3. Modelo orientado al control . . . . . . . . . . . . . . . . . 51

2.4. Modelo LPV considerando variación intra-paciente . . . . . . . . . . . . . . 52

2.5. Plataforma de simulación intervalar. . . . . . . . . . . . . . . . . . 53

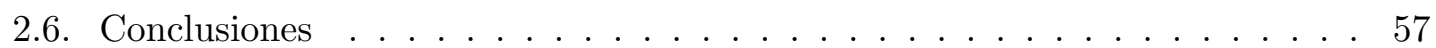


3. Estrategias de control a Lazo-Abierto vía limitación de IOB 59

3.1. Estimación de IOB . . . . . . . . . . . . . . . . . . . . . 60

3.2. Control glucémico posprandial . . . . . . . . . . . . . . 62

3.2.1. Marco teórico . . . . . . . . . . . . . . . . . 63

3.2.2. Super-Bolo automático basado en IOB . . . . . . . . . . . . . 65

3.2.3. Resultados . . . . . . . . . . . . . . . . . . 69

3.3. Control glucémico durante el ejercicio . . . . . . . . . . . . . 78

3.3.1. El ejercicio en la diabetes . . . . . . . . . . . . . . . . . 78

3.3.2. Modelos para la representación del ejercicio . . . . . . . . . . . . . 80

3.3.3. Propuesta de modelo . . . . . . . . . . . . . . . . . . . . 84

3.3.4. Mitigación del efecto rebote . . . . . . . . . . . . . . . . . 87

3.3.5. Resultados . . . . . . . . . . . . . . . . . . . 88

3.4. Conclusiones . . . . . . . . . . . . . . . . . . . 90

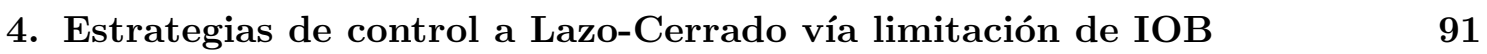

4.1. Algoritmo SAFE . . . . . . . . . . . . . . . . . . 92

4.2. Lazo híbrido: Algoritmo Time Enable . . . . . . . . . . . . . . . . . . 93

4.2.1. Ejemplo ilustrativo . . . . . . . . . . . . . . . . . . 95

$4.2 .2 . \quad$ Análisis in-silico . . . . . . . . . . . . . . . . . . . . . . . . 95

4.3. Lazo cerrado: Algoritmo ARG . . . . . . . . . . . . . . . . . . . . . . 98

4.3.1. Regulador LQG Conmutado. . . . . . . . . . . . . . . . . . . . . . . . . . . 99

4.3.2. Capa de Seguridad SAFE . . . . . . . . . . . . . . . . . 100

4.3.3. Mecanismo de conmutación del multicontrolador . . . . . . . . . . . 101

4.3.4. Módulos auxiliares . . . . . . . . . . . . . . . . . . . . . . 102

4.3.5. Ejemplo de operación . . . . . . . . . . . . . . . . 103

4.3.6. Pruebas Clínicas . . . . . . . . . . . . . . . . . . . . 104

4.4. Conclusiones . . . . . . . . . . . . . . . . . . . . . . . 110

5. Protección frente a hipoglucemias a lazo-cerrado vía limitación de la tasa $\begin{array}{ll}\text { de decrecimiento de la glucosa } & 111\end{array}$

5.1. Sistemas de prevención de hipoglucemias . . . . . . . . . . . . . . . . . . . . 111

5.2. Algoritmo GSAFE . . . . . . . . . . . . . . . . . . . . . . . 112

5.2.1. Predictor de Filtro de Kalman . . . . . . . . . . . . . . . 113

5.2.2. Adaptación de ganancia . . . . . . . . . . . . . . . . . . . 115

5.2.3. Implementación de la predicción de GP . . . . . . . . . . . . . . . 116

5.3. Ejemplos de aplicación del GSAFE . . . . . . . . . . . . . . . . . . . 117

5.3.1. Controlador MPC . . . . . . . . . . . . . . . . . 117

5.3.2. Controlador PDBasal+IFB . . . . . . . . . . . . . . . . . . 119

5.4. Resultados in-silico con diferentes controladores. . . . . . . . . . . . . . . 120

5.5. Conclusiones . . . . . . . . . . . . . . . . . . . . 122

\begin{tabular}{ll}
\hline 6. & Conclusiones y trabajo futuro \\
\hline
\end{tabular}

\begin{tabular}{ll}
\hline A. Lista de comidas mixtas & 127
\end{tabular}

\begin{tabular}{lr}
\hline B. Plataforma Android para ensayos clínicos & 129
\end{tabular}

B.1. Plataforma InsuMate . . . . . . . . . . . . . . . . . . . . . . . . . . . 129

B.2. Puesta en funcionamiento del sistema AAPS . . . . . . . . . . . . . 130

B.3. Resultados . . . . . . . . . . . . . . . . . . . . . . . . 131

B.4. Conclusiones . . . . . . . . . . . . . . . . . . . . . . . . . . . . . . .

\begin{tabular}{ll}
\hline Bibliografía & 135
\end{tabular} 


\section{Índice de figuras}

1.1. Diagrama de bloques conceptual de la regulación de la glucosa. . . . . . . . 4

1.2. Respuesta media de la glucosa en plasma, secreción de insulina y glucagón ante la ingesta en personas sanas y con DMT2. . . . . . . . . . . 5

1.3. Cantidad de personas con diabetes (20-79 años) en el mundo y proyecciones

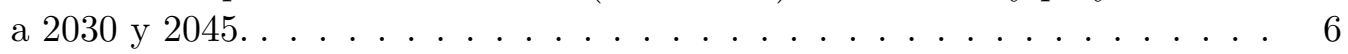

1.4. Sintomas y complicaciones de la diabetes. . . . . . . . . . . . . . . . 7

1.5. Suministro de insulina mediante inyecciones diarias. . . . . . . . . . . . 8

1.6. Aspectos del tratamiento de la diabetes. . . . . . . . . . . . . . . . . . 9

1.7. Ejemplo de tratamiento basal-bolo de un persona en su hogar mediante la

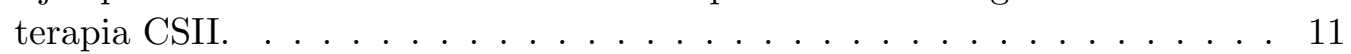

1.8. Evolución en el tiempo de la tecnología asociada a la diabetes. . . . . . . . 12

1.9. Representación esquemática del sensor subcutáneo de glucosa y del compartimento intersticial y el plasmático. . . . . . . . . . . . . . . 13

1.10. Desechos no reciclables generados en una semana por la tecnología utilizada por una persona con DMT1. . . . . . . . . . . . . . . . 17

1.11. Primera bomba de insulina en 1964 al actual diseño de sistema de PA portable. 18

1.12. Camino de 6 pasos del sistema de Páncreas Artificial. . . . . . . . . . . . . . 18

1.13. Taxonomía del diseño de sistemas de Páncreas Artificial. . . . . . . . . . . . 19

2.1. Diagrama de bloques que representa el modelo de Sorensen de 6 comparti-

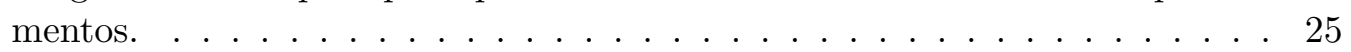

2.2. Diagrama de bloques que representa el modelo minimal de Bergman. . . . . 28

2.3. Diagrama de bloques que representa el modelo del grupo de Cambridge. . . 30

2.4. Diagrama del modelo de glucosa-insulina presentado por Dalla Man et al. . 34

2.5. Subsistema que representa a la glucosa $\ldots \ldots \ldots \ldots$. . . . . . . . . 35

2.6. Subsistema que representa a la insulina . . . . . . . . . . . . . . 35

2.7. Interfaz principal de la plataforma de simulación UNLP. . . . . . . . . . . . 44

2.8. Perfiles basales promedio por rango etario. . . . . . . . . . . . . . . . 47

2.9. Concentración de glucosa de un sujeto virtual incluyendo variación en la SI. 48

2.10. Media y desviación estándar del parámetro de variación aplicado sobre $V_{m x}$ sobre los 10 sujetos adultos. . . . . . . . . . . . . . . . . . 48

2.11. Perfiles de variación de SI para la clase virtual 5. . . . . . . . . . . . . . 49

2.12. Estructura del modelo $\mathrm{LPV}_{i}$ promedio. . . . . . . . . . . . . . 53

2.13. RMSE promedio entre las respuestas temporales del modelo $\mathrm{LPV}_{g}$ personalizado, $\mathrm{LPV}_{i}$ promedio y LPVi personalizado, comparados con el modelo no-lineal del UVA/Padova. . . . . . . . . . . . . . . . . . 53

2.14. Esquema de simulador intervalar. La incertidumbre afecta a diferentes componentes relacionados a la dinámica glucosa-insulina. . . . . . . . . . . . 54

2.15. Envolvente de la excursión de glucosa obtenida por el simulador intervalar. 56 
3.1. Representación de los diferentes tipos de insulina y sus curvas de absorción

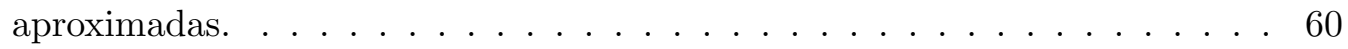

3.2. Curva ilustrativa del resultado teórico para el control posprandial presentado por Goodwin et al. 2015. . . . . . . . . . . . . . . . . . . . 64

3.3. Perfiles de IOB para diferentes sujetos generados a partir de un SB manual y del SB automático basado en IOB. . . . . . . . . . . . . . . . . 66

3.4. Tasa de aparición de glucosa de las comidas mixtas utilizadas en los esce-

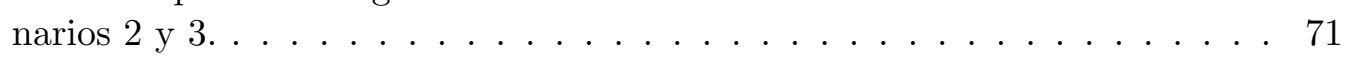

3.5. CVGA para el escenario de comida única de 25g. . . . . . . . . . . . . . . 72

3.6. CVGA para el escenario de comida única de 50g. . . . . . . . . . . . . . . 72

3.7. CVGA para el escenario de comida única de $75 \mathrm{~g}$. . . . . . . . . . . . . . . 73

3.8. CVGA para el escenario de comida única de 100g. . . . . . . . . . . . . . 73

3.9. Respuesta de glucosa media \pm DE para los 10 adultos en el escenario de

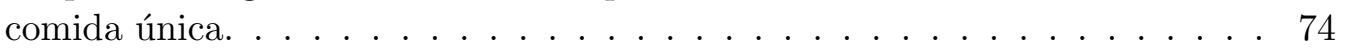

3.10. Respuesta de glucosa media \pm DE para los 10 adolescentes en el escenario de comida única. . . . . . . . . . . . . . . . 74

3.11. Respuesta media de glucosa \pm DE para los 10 niños en el escenario de comida única. . . . . . . . . . . . . . . . . . . . 75

3.12. CVGA para los escenarios de un solo día. Comparación entre los tratamientos en adultos (marcadores cuadrados), adolescentes (marcadores romboidales) y niños (marcadores circulares).$\ldots \ldots \ldots 76$

3.13. Respuesta media de glucosa \pm DE para los escenarios de un día. La línea azul a rayas corresponde al tratamiento estándar, la línea naranja sólida al SB automático. El área gris indica el rango euglucémico [70-180]mg/dl.] . . . 77

3.14. Beneficios, motivaciones y barreras de la actividad física en el manejo de la

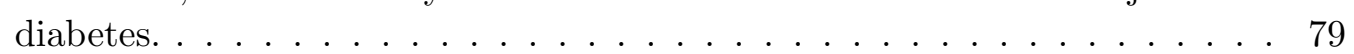

3.15. Variabilidad individual en las respuestas de la glucosa en sangre a las dife-

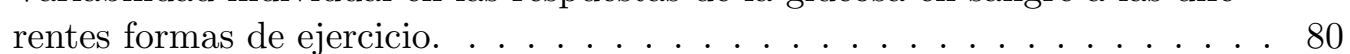

3.16. Motivos por los que se produce el efecto de rebote en la glucosa en plasma luego o durante el ejercicio en la DMT1. . . . . . . . . . . . . . . . . 8 81

3.17. Ejemplo de cambio de sensibilidad insulínica e la simulación durante el

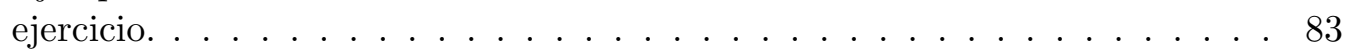

3.18. Representación en bloques del modelo UVA/Padova con remarques en las dinámicas que se afectan durante el ejercicio mediante lo propuesto por Roy y Parker 2007 y Schiavon et al 2013. . . . . . . . . . . . . . . . . . 85

3.19. Hormonas y metabolitos medidos antes, durante y después de diferentes intensidades de ejercicio en adolescentes con diabetes y controles. . . . . . . 86

3.20. Simulación Monte Carlo con barrido en $K_{\alpha}$ y $u_{e x}$ para cada terapia propuesta durante el ejercicio. . . . . . . . . . . . . . . . . . . . 89

4.1. Diagrama en bloques de la capa de seguridad SAFE. . . . . . . . . . . . . 92

4.2. Diagrama de bloques que representa un lazo híbrido de control de glucosa junto a la capa SAFE. . . . . . . . . . . . . . . . . . . 94

4.3. Señales principales del modo de operación Time Enable sobre el Adulto \#1 del simulador UVA/Padova ante una comida de $60 \mathrm{~g}$ de CHO. . . . . . . . . 95

4.4. Los límites $\overline{I O B}_{\min }$ y $\overline{I O B}_{\max }$ ilustrados de acuerdo al perfil de IOB en el tiempo de un bolo de $6 \mathrm{U}$ de insulina. . . . . . . . . . . . . . . . . 996

4.5. Reunión de trabajo junto a, de izquierda a derecha, Dra. Marcela Moscoso, Dr. Hernán De Battista, Ing. Nicolás Rosales, Ing. Emilia Fushimi, Dr. Fabricio Garelli y Dr. Patricio Colmegna. . . . . . . . . . . . . . . . . 99

4.6. Sistema a lazo cerrado con el algoritmo ARG. . . . . . . . . . . . . . . . 99 
4.7. Respuesta de lazo cerrado del algoritmo ARG para un adulto in-silico ante la ingesta de una comida mixta. . . . . . . . . . . . . . . . . . . 104

4.8. Los 5 participantes con sus respectivos smartphones con la plataforma DiAs y el algoritmo ARG corriendo en ella. . . . . . . . . . . . . . . 106

4.9. Línea de tiempo del protocolo realizado durante los ensayos clínicos. . . . . 107

4.10. Tiempo en rango cumulativo para los 5 sujetos en LA y LC para los periodos

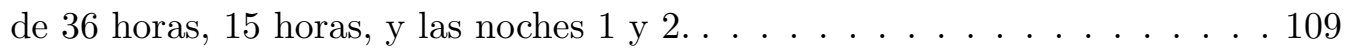

4.11. Excursión promedio de CGM para los 5 sujetos en LA y LC durante el periodo de 15 horas. . . . . . . . . . . . . . . . . . . 109

5.1. Diagrama de bloques de la estructura de control propuesta. . . . . . . . . . 113

5.2. Proceso SISO con estados expandidos para representar el retardo $d$. . . . . 114

5.3. Ejemplo ilustrativo de la capa de GSAFE sobre un controlador MPC en un

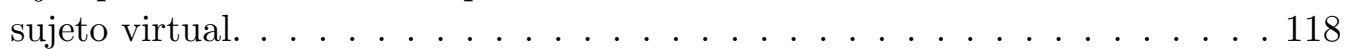

5.4. Ejemplo ilustrativo de la capa de GSAFE sobre un controlador PDBasal+IFB en un sujeto virtual. . . . . . . . . . . . . . . . . . . 120

B.1. Sistema AAPS: Bomba, Android APS, receptor del sensor y NightScout . . 130

B.2. Dispositivos y aplicaciones del sistema Android APS. . . . . . . . . . . . . . 131

B.3. Plataforma InsuMate durante su ensayo corriendo el algoritmo ARG. . . . . 132

B.4. Equipo de trabajo colocando y configurando el sensor Dexcom G4 para el ensayo de la plataforma. . . . . . . . . . . . . . . . . . . 133

B.5. Línea tempral de los resultados de la plataforma InsuMate ante diferentes

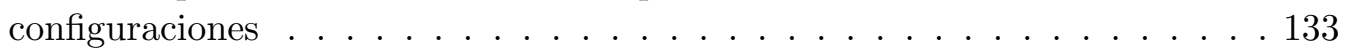





\section{Índice de tablas}

1.1. CGM disponibles actualmente en el mercado. . . . . . . . . . . . . . . . . . 14

1.2. Bombas de insulina disponibles en el mercado . . . . . . . . . . . . . . . . 15

2.1. Comparación de los modelos del sistema glucosa-insulina presentados en la

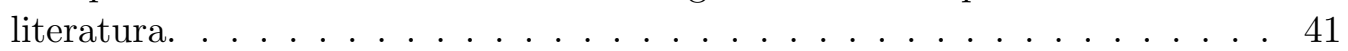

2.2. Clases de patrones de sensibilidad insulínica. . . . . . . . . . . . . . . 49

2.3. Parámetros de los modelos de cada subsistema a los que se les adjudicó incertidumbre en el simulador intervalar. . . . . . . . . . . . . . . . 55

3.1. Parámetro $K_{\text {DIA }}\left(\min ^{-1}\right)$ del modelo dinámico de IOB para diferentes duraciones de acción de insulina. . . . . . . . . . . . . . . . . . . . . 61

3.2. Comparación entre el tratamiento estándar y el SB automático para los escenarios de una comida única. . . . . . . . . . . . . . . . . 71

3.3. Comparación entre el tratamiento estándar y el SB automático para los escenarios de un día. . . . . . . . . . . . . . . . . 71

3.4. Valores medios de los parámetros de las dinámicas propuestas por Roy y Parker. . . . . . . . . . . . . . . . . . 84

4.1. Barrido de $\overline{I O B}$ para los 30 sujetos virtuales utilizando $k_{L A}=1$. . . . . . . 97

4.2. $\quad$ Barrido de $\overline{I O B}$ para los 30 sujetos virtuales utilizando $k_{L A}=0,7$. . . . . . 97

4.3. Barrido de $\overline{I O B}$ para los 30 sujetos virtuales utilizando $k_{L A}=0,5$. . . . . . 97

4.4. Barrido de $k_{p}$ sobre los 30 sujetos virtuales. PDBasal en lazo-híbrido utilizando $k_{L A}=0,7$ con y sin el algoritmo Time Enable. . . . . . . . . . . . 98

5.1. Resultados poblacionales: controlador MPC . . . . . . . . . . . . . . . . . 121

5.2. Resultados poblacionales: controlador PDBasal+IFB . . . . . . . . . . . . 121

5.3. $\quad$ Resultados poblacionales: SAFE vs. GSAFE . . . . . . . . . . . . . . . 121

A.1. Biblioteca de comidas mixtas. . . . . . . . . . . . . . . . . . . . . 128 



\section{Abreviaciones}

\section{Diabetes en general}

AF Actividad Física

AIG Alto Índice Glucémico

BIG Bajo Índice Glucémico

CHO Carbohidratos

DM Diabetes Mellitus

DMT1 Diabetes Mellitus Tipo 1

DMT2 Diabetes Mellitus Tipo 2

GP Glucosa en Plasma

HbA1c Hemoglobina Glicosilada

HR Heart Rate

IMC Índice de Masa Corporal

PA Páncreas Artificial

PEG Producción Endógena de Glucosa

SI Sensibilidad Insulínica

SMBG Self-Monitoring of Blood Glucose

\section{Tratamientos}

BR Basal Rat ${ }^{1}$

BOB Bolus On Board

CF Correction Factor

CGM Continuous Glucose Monitor

\footnotetext{
${ }^{1}$ Algunas definiciones corresponden a las siglas en inglés de los términos. Se utilizaron abreviaciones en inglés cuando representan nombres técnicos o se encuentran difundidas de esa manera incluso en la literatura en español.
} 


$\begin{array}{ll}\text { CR } & \text { Carbohidrate Ratio } \\ \text { CSII } & \text { Continuous Subcutaneous Insulin Infusion } \\ \text { DIA } & \text { Duration of Insulin Action } \\ \text { FDA } & \text { Food and Drugs Administration } \\ \text { HBGI } & \text { High Blood Glucose Index } \\ \text { IOB } & \text { Insulin-On-Board } \\ \text { LBGI } & \text { Low Blood Glucose Index } \\ \text { LGS } & \text { Low Glucose Suspend } \\ \text { MARD } & \text { Mean Absolute Relative Difference } \\ \text { MDI } & \text { Multiple Daily Injections } \\ \text { OB } & \text { Optimal Bolus } \\ \text { PLGS } & \text { Predictive Low Glucose Suspend } \\ \text { SAP } & \text { Sensor-Augmented Pumps } \\ \text { SB } & \text { Super-Bolo } \\ \text { TDI } & \text { Total Daily Insulin }\end{array}$

\section{Control}
AMP Announced Meal Predictor
ARG Automatic Regultaion of Glucose
CVGA Control Variability Grid Analysis
GSAFE Glucose-based Safety Auxiliary Feedback Element
IFB Insulin Feedback
KF Kalman Filter
KFP Kalman Filter Predictor
LPV Linear Parameter-Varying
LQG Linear Quadratic Gaussian
LTI Linear Time Invariant
MPC Control Predictivo basado en Modelo
PID Proporcional-Integral-Derivativo
RMSE Relative Mean Square Error
SAFE Safety Auxiliary Feedback Element
SISO Single-Input-Single-Output 
SLQG Switched Linear Quadratic Gaussian

SM Sliding-Mode

SMRC Sliding-Mode Reference Conditioning

UMP Unannounced Meal Predictor

\section{Instituciones}

ADA American Diabetes Association

ANMAT Administración Nacional de Medicamentos, Alimentos y Tecnología Médica

ATTD Advanced Technologies and Treatments of Diabetes

FAD Federación Argentina de Diabetes

HIBA Hospital Italiano de Buenos Aires

IFD International Federation of Diabetes

ITBA Instituto Tecnológico de Buenos Aires

JDRF Juvenile Diabetes Research Foundation

LEICI Instituto de Investigaciones en Electróncia, Control y Procesamiento de Señales

UNLP Universidad Nacional de La Plata 



\section{Prefacio}

Lo que me cautivó de la ingeniería electrónica en un primer lugar es su amplio campo de aplicación. En particular, remontándome ya tiempo atrás desde el grado, la rama de Bioingeniería me parecía casi irreal, una cercanía a la ciencia ficción. El equilibrio y funcionamiento de los organismos biológicos no dejan de maravillarme y preguntarme hasta donde seremos capaces de llegar. El haber estudiado en la UNLP me dio la gran oportunidad de hacer un postgrado en esta orientación, de darme un lugar y cobijo para poder adentrarme en una de las aristas de la Bioingeniería. Quisiera reconocer que el puntapié inicial, además de la charla con Hernán y Fabricio, fue el artículo [1], al cual le tengo cierto cariño (por eso merece ser la primera cita en este gran recorrido).

El control automático de glucosa en sangre nace a partir de una necesidad de la igualdad y tranquilidad para millones de personas. Aunque hoy en día la tecnología se encuentra lejos de alcanzar a todas aquellas personas que tienen Diabetes Mellitus Tipo 1 (DMT1), el estudio y desarrollo de múltiples aspectos entorno a la misma han crecido vertiginosamente en las ultimas décadas, más aún en éstas primeras dos del siglo XXI.

Como en cualquier problema de ingeniería, en primer lugar se estudiaron los modelos matemáticos más relevantes que describan la dinámica glucosa-insulina principalmente. Comprender y analizar las dinámicas en juego es fundamental. A partir de la elección de uno de los múltiples modelos disponibles en la literatura, se propone una plataforma de simulación que contemple incertidumbres, nuevas tecnologías, múltiples escenarios. Se apuntó también a la versatilidad para su utilización y adaptación, resultando intuitiva para el uso por parte de colegas y personas que trabajen en el tema.

La segunda parte de la tesis hace enfoque a esquemas de control para el tratamiento de la diabetes a partir de dos enfoques: control a lazo abierto y control a lazo cerrado. El control adecuado de la glucosa es esencial para mejorar la calidad de vida de las personas con DMT1. El principal desafío parte de que el sistema presenta saturación en la actuación, ruido de medición, la gran variabilidad intra- e inter-paciente, retardos debido al uso de la ruta subcutánea y enormes perturbaciones debido a la ingesta. A su vez, se desea minimizar la interacción de la persona en el control de su glucosa, por lo que se buscaron alternativas a la tecnología que ya disponen y se propusieron técnicas para el control de lazo cerrado puro.

Por un lado, se realizan dos propuestas para el control a lazo abierto vía la limitación de la insulina disponible en el cuerpo (Insulin-On-Board, IOB). Se propone un algoritmo para el control posprandial el cual permite el cómputo automático de un super-bolo, en base a técnicas registradas en patente por los directores. Este permite compensar comidas de alto índice glucémico a partir de la automatización de una técnica conocida. A su vez, se propone un algoritmo para la realización de actividad física, teniendo en cuenta tanto el miedo a las hipoglucemias por parte de la comunidad, como así evitar una hiperglucemia severa.

Por otro lado, se proponen técnicas de control de lazo cerrado híbrido y puro vía 
limitaciones de IOB. Se presenta una propuesta para regular el paso entre el control a lazo abierto (el suministro de un bolo para compensar una comida) al lazo cerrado mediante una técnica basada en control por modos deslizantes (Sliding Mode, SM) en vista a la seguridad durante ensayos clínicos. Se detalla la participación en el desarrollo del algoritmo ARG, realizado en colaboración entre la UNLP y el ITBA y ensayado clínicamente en 2017 en el Hospital Italiano de Buenos Aires.

Por último, se propone un esquema de lazo cerrado puro vía limitación de la tasa de decrecimiento de la glucosa. Aquí se propone limitar la variable controlada mediante la adaptación del controlador, para evitar hipoglucemias debido a su sobre-actuación, partiendo de técnicas como SM, filtro de Kalman y un controlador principal con estructura MPC y otro PID.

Todas las propuestas fueron ensayadas y validadas de forma in-silico en la plataforma desarrollada, la cual se encuentra basada en el simulador UVA/Padova, el cual fue aceptado como reemplazo de pruebas en animales por la Food and Drug Administration.

\section{Objetivos}

Siendo esta la primera tesis de la Universidad Nacional de La Plata en esta temática, se proponen los siguientes objetivos involucrados en el tratamiento de la diabetes y en el control de la glucosa:

1. Relevar el estado del arte del desarrollo tanto de tratamientos y tecnologías para la diabetes como de los sistemas de Páncreas Artificial.

2. Diseñar una plataforma de simulación versátil y realista en base a modelos validados de la dinámica glucosa-insulina.

3. Diseñar estrategias de control que abarquen los diferentes aspectos que involucra el control glucémico: técnicas a lazo abierto, lazo híbrido y lazo cerrado.

\section{Contribuciones}

Muchos de los resultados que aparecen en esta tesis fueron presentados con anterioridad en las siguientes publicaciones:

\section{Artículos en revista}

1. Rosales N., Vehí J., Garelli F., De Battista H. Open-loop glucose control: Automatic IOB-based super-bolus feature for commercial insulin pumps. Computer Methods and Programs in Biomedicine. Vol. 159, Pág. 145-158. DOI 10.1016/j.cmpb.2018.03.007. 2018.

2. Fushimi E., Rosales N., De Battista H, Garelli F. Artificial pancreas clinical trials: Moving towards closed-loop control using insulin-on-board constraints. Biomedical Signal Processing and Control. Vol. 45, Pag. 1-9. DOI 10.1016/j.bspc.2018.05.009. 2018.

3. Sánchez-Peña R., Colmegna P., Garelli F., De Battista H., García-Violini D., MoscosoVásquez M., Rosales N, Fushimi E., Campos-Náñez E., Breton M., Beruto V., Scibona P., Rodriguez C., Giunta J., Simonovich V., Belloso W., Cherñavvsky D., Grosembacher L.. Artificial Pancreas: Clinical study in Latin America Without Premeal Insulin Boluses. Journal of Diabetes Science and Technology. Vol. 12, no. 5, Pag. 914-925. DOI 10.1177/1932296818786488 2018. 
4. Colmegna P., Garelli F., Fushimi E., Moscoso-Vásquez M., Rosales N., García-Violini D., De Battista H., Sánchez-Peña R., Artificial pancreas: The argentine experience. Science Reviews. Vol. 1, no. 1, Pag 37-53. 2019.

5. Moscoso-Vásquez M., Colmegna P., Sánchez-Peña R., Rosales N., Garelli F. ControlOriented Model with Intra-Patient Variations for an Artificial Pancreas. IEEE Journal of Biomedical and Health Informatics. DOI 10.1109/JBHI.2020.2969389. 2020.

6. Rosales N., De Battista H., Garelli F. Hypoglycemia prevention: Controller adaptation for glucose rate limiting in Artificial Pancreas System. Enviado a IEEE Journal of Biomedical and Health Informatics. 2020.

\section{Artículos en congresos internacionales}

1. Rosales N., Garelli F., De Battista H. Automatic Super-Bolus and Bolus-Shaping Features for Insulin Pumps. 10th International Conference on Advanced Technologies \& Treatments for Diabetes (ATTD). Publicación de resumen; referato de resumen extendido. Diabetes Technology \& Therapeutics. Vol. 19, No. S1. Paris. DOI 10.1089/dia.2017.2525.abstracts. 2017.

2. Garelli F., Fushimi E., Rosales N., De Battista H. Open to closed-loop transitions schemes for in vivo glucose control. 10th International Conference on Advanced Technologies \& Treatments for Diabetes (ATTD). Publicación de resumen; referato de resumen extendido. Paris. Diabetes Technology \& Therapeutics. Vol. 19, No. S1. DOI 10.1089/dia.2017.2525.abstracts. 2017.

3. Rosales N., León Vargas F. M., Garelli F., De Battista H. Hypoglycemia Prevention in Closed-loop Glycemic Control via Constraints in the Glucose Slope. 11th International Conference on Advanced Technologies \& Treatments for Diabetes (ATTD). Publicación de resumen; referato de resumen extendido. Viena. Diabetes Technology \& Therapeutics. Vol. 20, No. S1. DOI 10.1089/dia.2018.2525.abstracts. 2018.

4. Rosales N., Vehí J., De Battista H., Garelli F. Modelling and Regulation of the Effects of Long-Duration Medium-Intensity Exercise in Type 1 Diabetic Patients. 12th International Conference on Advanced Technologies \& Treatments for Diabetes (ATTD). Publicación de resumen; referato de resumen extendido. Berlin. Diabetes Technology \& Therapeutics. Vol. 21, No. S1. DOI doi.org/10.1089/dia.2019.2525.abstracts. 2019 .

5. García-Jaramillo M., León-Vargas F., Rosales N., Molano A., Garelli F. Interval Simulator of the Glucose-Insulin System. Applied Computer Sciences in Engineering. WEA 2019. Communications in Computer and Information Science, vol 1052. DOI 10.1007/978-3-030-31019-6_58. 2019.

6. Arambarri D., Mendoza L., Rosales N., Garelli F. Glucontrol: Implementation and Testing of Open Source Platform for APS Clinical Trials. 13th International Conference on Advanced Technologies \& Treatments for Diabetes (ATTD). Publicación de resumen; referato de resumen extendido. Madrid. Diabetes Technology \& Therapeutics. Vol. 22, No. S1. DOI 10.1089/dia.2020.2525.abstracts. 2020.

\section{Artículos en congresos nacionales}

1. Rosales N., Garelli F., De Battista H. T1DM glycemic control: Effects of mixed meals and intra-patient variability in continuous insulin treatments XVI Reunión de trabajo en Procesamiento de la Información y Control (RPIC). UTN-UNC-CONICET. Córdoba. ISBN 978-1-4673-8466-7. 2015. 
2. Rosales N., Garelli F., De Battista H. Automatic Super-Bolus Feature for Commer-

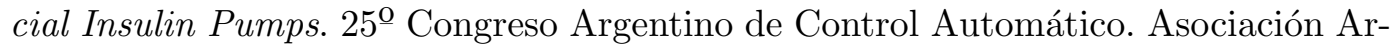
gentina de Control Automático (AADECA). Buenos Aires. ISBN 978-950-99994-9-7. 2016 .

3. Fushimi E., Rosales N., Garelli F., De Battista H. Open to Closed-Loop Transition Schemes for In Vivo Glucose Control. 25ํㅡㄹ Congreso Argentino de Control Automático. Asociación Argentina de Control Automático (AADECA). Buenos Aires. ISBN 978-950-99994-9-7. 2016.

4. Rosales N., De Battista H, Garelli F. Artificial Pancreas: hypoglycemia prevention via constraints in the glycemia rate of change. XVII Reunión de trabajo en Procesamiento de la Información y Control (RPIC). UNMDP-ICYTE-CONICET. Mar del Plata. ISBN 978-987-544-754-7. 2017.

5. Fushimi E., Rosales N., De Battista H, Garelli F. Constraints on the Insulin Infusion for Artificial Pancreas Clinical Trials. XVII Reunión de trabajo en Procesamiento de la Información y Control (RPIC). UNMDP-ICYTE-CONICET. Mar del Plata. ISBN 978-987-544-754-7. 2017.

6. Rosales N., De Battista H, Garelli F. Plasma Glucose Prediction and its Application to Low Glucose Suspension Systems. 26ํㅡㄹ Congreso Argentino de Control Automático. Asociación Argentina de Control Automático (AADECA). Buenos Aires. ISBN 9789-8746-8591-9. 2018.

7. Rosales N., Vehí J., De Battista H, Garelli F. Modelado y mitigación in-silico del efecto "rebote" durante el ejercicio en la diabetes tipo 1. XVIII Reunión de trabajo en Procesamiento de la Información y Control (RPIC). UNS-CONICET. Bahia Blanca. 2019.

\section{Organización}

Esta tesis se organiza de la siguiente manera:

- En el capítulo 1 se desarrolla el estado del arte, comenzado por definir la diabetes y sus problemáticas, los tratamientos y la tecnología circundante, hasta llegar al concepto de Páncreas Artificial y el control de glucosa de lazo cerrado.

- En el capítulo 2 de detallan y comparan los principales modelos matemáticos del sistema glucosa-insulina de la literatura. Luego se desarrolla la implementación de una plataforma de simulación para el ensayo de estrategias de control de glucosa de forma in-silico. En esta se consideran diferentes perfiles de variación intra-paciente y comidas mixtas. A su vez se proponen otras formas de modelado, considerando técnicas intervalares y un modelo de orden reducido orientados al control que cubra variaciones intra-paciente.

- En el capítulo 3 se proponen estrategias de control a lazo abierto mediante la terapia con bombas de insulina para dos casos: el control posprandial y el control durante el ejercicio. Los algoritmos presentados se basan en la estimación y regulación de la insulina a bordo, siendo aquella que se encuentra en el espacio subcutáneo disponible para actuar.

- En el capítulo 4 se detallan estrategias de control a lazo cerrado, diseñadas también a partir de la insulina a bordo y la implementación de la capa SAFE. 
Se presenta el caso híbrido (con suministro de bolos) y el caso de lazo cerrado correspondiente al algoritmo ARG. Este último fue desarrollado en conjunto con el ITBA y la UNLP. Se presentará el caso de estudios y los ensayos clínicos realizados en el país.

- En el capítulo 5 se presenta una estrategia de control para el lazo cerrado puro. Esta se centra en la prevención de hipoglucemias en base a la limitación de la tasa de decrecimiento de la glucosa en plasma.

- Las conclusiones finales y el trabajo futuro son presentadas en el capítulo 6 .

- Por último, se añade un apéndice con el desarrollo e implementación de una plataforma basada en Android para realizar ensayos clínicos. 


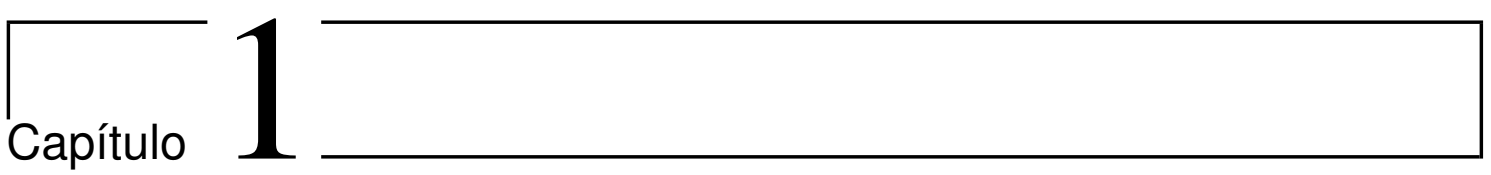

\section{Introducción}

La Diabetes afecta a millones de personas en el mundo. Resulta un desafío cotidiano para quien la padece y puede tener consecuencias tanto a corto plazo como a largo, debido a un tratamiento inadecuado. El avance tecnológico reciente (con respecto a la longevidad de la afección) aumentó considerablemente la calidad de vida de quienes deben lidiar con la diabetes, aunque lamentablemente no todos y todas tienen acceso a las mismas herramientas.

Desde la perspectiva de la ingeniería de control automático, la propuesta de desarrollar un control de lazo cerrado de glucosa para personas insulino-dependientes propone un gran desafío a nivel teórico, debido a los retardos y restricciones que presenta la planta, y a su vez el desafío práctico de implementar sobre las tecnologías existentes

El problema no sólo es desafiante a nivel teórico y práctico, sino también a nivel social. Muchas personas con diabetes confían en lograr una vida mejor y no están esperando (\#WeAreNotWaiting).

\subsection{Diabetes Mellitus}

La Diabetes Mellitus (DM) consiste en un trastorno metabólico que se manifiesta de forma crónica, caracterizándose por concentraciones elevadas de Glucosa en Plasma (GP). En la Figura 1.1 se muestra un diagrama de cómo el cuerpo regula y utiliza la glucosa. La principal causa de la diabetes se debe a la baja o nula producción de la hormona insulina por parte del cuerpo, o la pérdida de sensibilidad hacia a misma. La insulina es una hormona anabólica secretada por las células $\beta$ de los Islotes de Langerhans del páncreas. $\mathrm{Su}$ principal función es el aprovechamiento de nutrientes y es la llave para proveer de energía a las células de los tejidos. Otras hormonas participan de la regulación de la glucosa, siendo el glucagón una de las principales junto a la insulina, hormona secretada por la células $\alpha$ del páncreas. Esta hormona estimula la liberación de glucosa por parte del hígado para aumentar la concentración de GP

En una persona sana se pueden determinar los siguientes rangos de glucemia: ayuno $\mathrm{GP} \in[70,100] \mathrm{mg} / \mathrm{dl}$ y posprandial $\left.\right|^{1} \mathrm{GP}<150 \mathrm{mg} / \mathrm{dl}$. Estos rangos cambian para una persona con diabetes, considerándose como normoglucemia o euglicemia $\mathrm{GP} \in[70,180] \mathrm{mg} / \mathrm{dl}$. Debe notarse que se considera un rango más amplio debido a la dificultad de control glucémico. Niveles de GP por debajo de este rango se denomina hipoglucemia y por encima hiperglucemia.

El diagnostico de la DM debe realizarse en un entorno médico (como el consultorio de

\footnotetext{
${ }^{1}$ Se considera como periodo posprandial de 2 a 4 horas después de comer, siendo de gran importancia mantener una buena regulación de la glucemia durante ese periodo.
} 


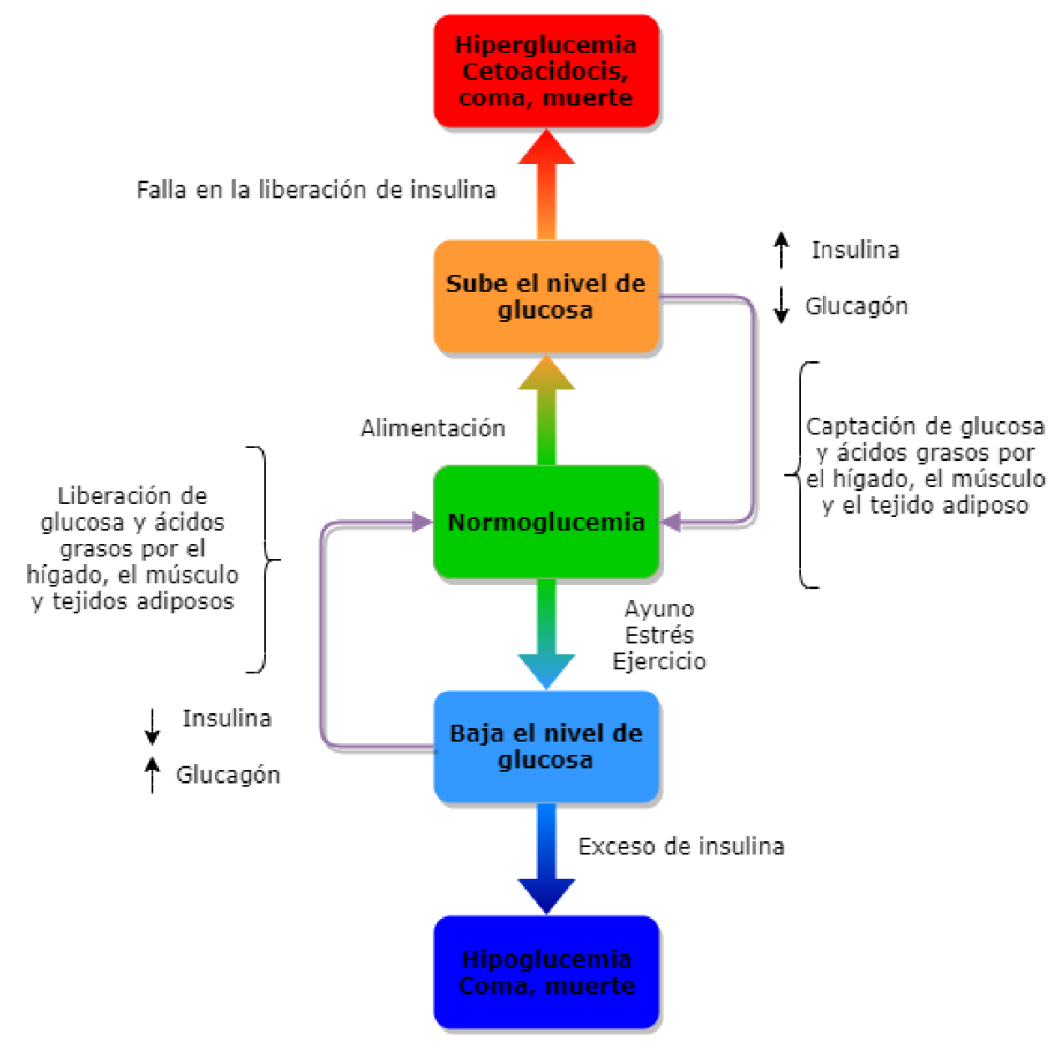

Figura 1.1: Diagrama de bloques conceptual de la regulación de la glucosa.

su médico o un laboratorio) y puede realizarse por medio de diferentes vías, de acuerdo a [2]:

- Prueba aleatoria de GP $\geq 200 \mathrm{mg} / \mathrm{dl}$ en cualquier momento del día.

- Glucemia en ayunas (de al menos 8 horas) $\geq 126 \mathrm{mg} / \mathrm{dl}$.

- Glucemia $\geq 200 \mathrm{mg} / \mathrm{dl}$ a las 2 horas en una prueba de tolerancia a la glucosa oral.

- Hemoglobina Glucosilada (HbA1c mayor o igual de 6,5\%).

La mayoría de los casos de diabetes pueden englobarse en dos grandes categorías, pero cabe destacar una tercera que tiene un importante grado de incidencia:

- Diabetes Mellitus Tipo 1 (DMT1): Se suele manifestar a temprana edad, generalmente antes de los 35 años. No existe la producción endógena de insulina debido a una destrucción autoinmune de las células $\beta$. Quienes la padecen son personas insulino-dependientes.

- Diabetes Mellitus Tipo 2 (DMT2): Suele desarrollarse en adultos (después de los 35-40 años) y se la relaciona a diversos factores, como la falta de actividad física o la obesidad. Disminuye la producción de insulina o el cuerpo genera resistencia a la misma. En algunos casos, se requiere suministro exógeno de insulina.

- Diabetes Mellitus gestacional: se manifiesta durante el periodo gestacional en más del $8 \%$ de los embarazos.

Existen otros tipos de diabetes, que pueden inducirse debido a infecciones, efectos colaterales de drogas, síndromes genéticos, entre otros factores aún en estudio. 


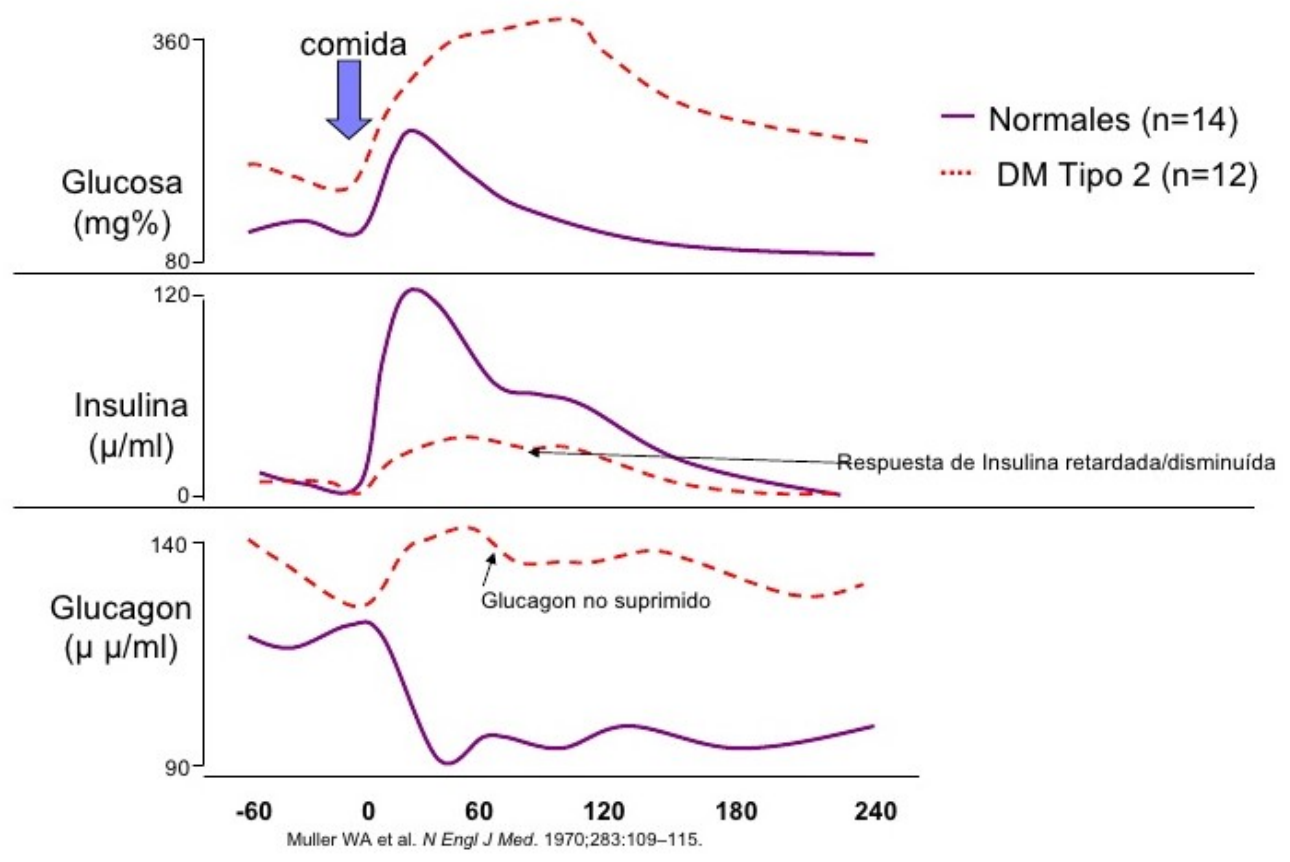

Figura 1.2: Respuesta media de la glucosa en plasma, secreción de insulina y glucagón ante la ingesta en personas sanas y con DMT2.

En la Figura 1.2 puede observarse la respuesta media ante la ingesta de la glucosa, insulina y glucagón en plasma de personas sana y con diabetes tipo $2[3]$. En una persona con DMT1 el caso es aún más grave ya que no tiene respuesta de insulina ante la ingesta, por lo que la glucosa crece a niveles muy altos y peligrosos para la salud.

\subsection{La vida con Diabetes}

Cuando una persona tiene DM, su cotidianidad se ve envuelta en el control intensivo sobre su cuerpo. Eventos como comer, dormir, correr, dar una clase, enfrentarse a un trabajo, y muchas otras actividades se vuelven desafíos que escapan a las demás personas. Cuando uno se encuentra a cargo del control de una niña o niño con diabetes las implicaciones son aún mayores. La mayor preocupación al tratar este trastorno es el peligro latente a las hipoglucemias [5]. En muchos países, especialmente aquellos con familias con economía precaria, el acceso a la insulina y herramientas de cuidado, como así también a una educación estructurada de la diabetes, es limitado.

La Federación Internacional de Diabetes (International Federation of Diabetes (IFD) promueve la investigación de la diabetes desde 1950. Cada dos años publica un atlas con toda clase de información relacionada a la diabetes. Según su último informe [4], la diabetes constituye el tercer factor de riesgo en importancia como causa de muerte a nivel global. Se estima que en el mundo el número total de individuos que padecen diabetes se elevará de 463 millones de personas en el 2019 a 700 millones en el año 2045. A nivel global y en particular en Argentina, donde hay 4 millones de diabéticos, aproximadamente el $10 \%$ de los estos padecen DMT1, En la Fig. 1.3 se visualiza la cantidad de personas con diabetes para un rango de edad de 20-79 años en diferentes regiones del mundo y su estimación a futuro.

En uno de los mayores estudios realizados hasta la fecha sobre las tendencias de la diabetes [4], los investigadores indicaron que el envejecimiento de la población y el aumento de la obesidad en el mundo implican que la enfermedad se esté convirtiendo en un tema decisivo para la salud pública global. En América Latina (exceptuando México) se estima 


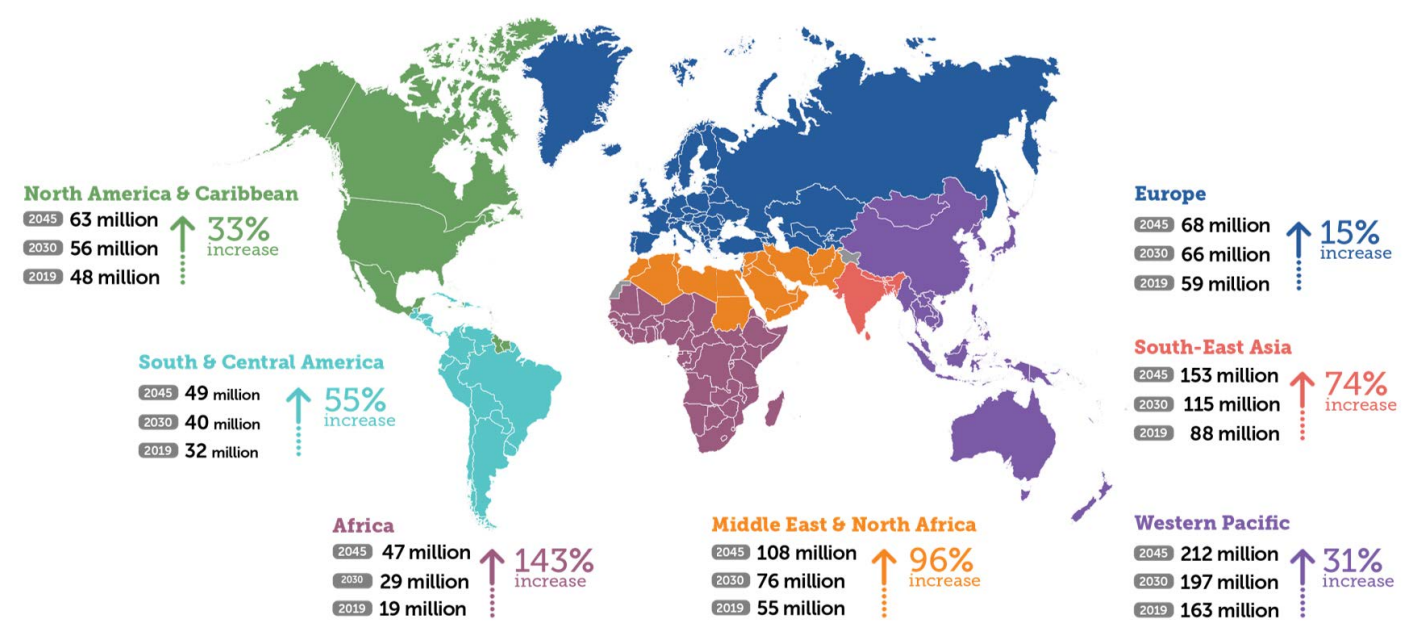

Figura 1.3: Cantidad de personas con diabetes (20-79 años) en el mundo y por región en el año 2019 y proyecciones a 2030 y 2045 [4.

que el 9.4\% de los adultos entre 20-79 años tiene diabetes en 2019 (31.6 millones) y más del $40 \%$ no se encuentra diagnosticado. A su vez, en este mismo año se estima que murieron 243.000 personas en ese rango de edad debido a complicaciones de la diabetes. Más del $50 \%$ de las muertes ocurren en Brasil, un país con mucha desigualdad a pesar de ser una de las economías mas grandes del mundo. En el 2019 el gasto relacionado a la diabetes en la región fue de 69.7 mil millones de dolares, que sólo se corresponde al $9 \%$ del total de la inversión global. Los países que más invierten de su gasto en salud son Cuba (24.3\%), Brasil $(24.2 \%)$ y Costa Rica $(21.3 \%)$, mientras que donde menos se gasta es en Argentina (5\%) y Uruguay (6.1\%). Aún así, el Plan Argentina Innovadora 2020 identificó dentro del apartado Salud a las Enfermedades crónicas, complejas con componentes multigénicos y asociadas a adultos como uno de sus Núcleos Socio Productivos Estratégicos [6].

De acuerdo a la Cuarta Encuesta Nacional de Factores de Riesgo [7], la prevalencia autorreportada de glucemia elevada o diabetes aumentó de $9,8 \%$ a $12,7 \%$. Se considera que ese aumento significativo era esperable dado que acompaña el crecimiento de la obesidad y de la inactividad física, ambos factores de riesgo reconocidos de DMT2. Es decir que, para reducir la prevalencia de diabetes es fundamental implementar las políticas de prevención y control de la obesidad. Las políticas en marcha en nuestro país son:

- La diabetes también constituye una línea de cuidado priorizada en el marco de la CUS y en los Programas de Financiamiento Internacional, como REDES, PROTEGER y PACES. De igual modo, a través del Programa Medicamentos Esenciales se entregan fármacos e insumos para el tratamiento de la diabetes en el Primer Nivel de Atención.

- El Programa Nacional De Prevención Y Control De Personas Con Diabetes Mellitus creado por la Resolución 1156 /2014, junto con el Área de Servicios de Salud de la DNPSyCENT, otras áreas de la Secretaria de Gobierno de Salud, los Programas Provinciales, y en colaboración con organizaciones científicas y académicas, está desarrollando la actualización de la Guía De Práctica Clínica de Diabetes Mellitus, cuya publicación estaba originalmente prevista para el segundo semestre de 2019. Del mismo modo, se están llevando a cabo capacitaciones destinadas a los equipo de salud del Primer Nivel de Atención sobre la temática de la diabetes.

- Desde el Programa Nacional De Prevención Y Control De Personas Con Diabetes Mellitus y la Comisión Nacional Permanente Asesora de Diabetes se brindó asistencia técnica para la actualización de las normas de provisión de medicamentos e insumos 


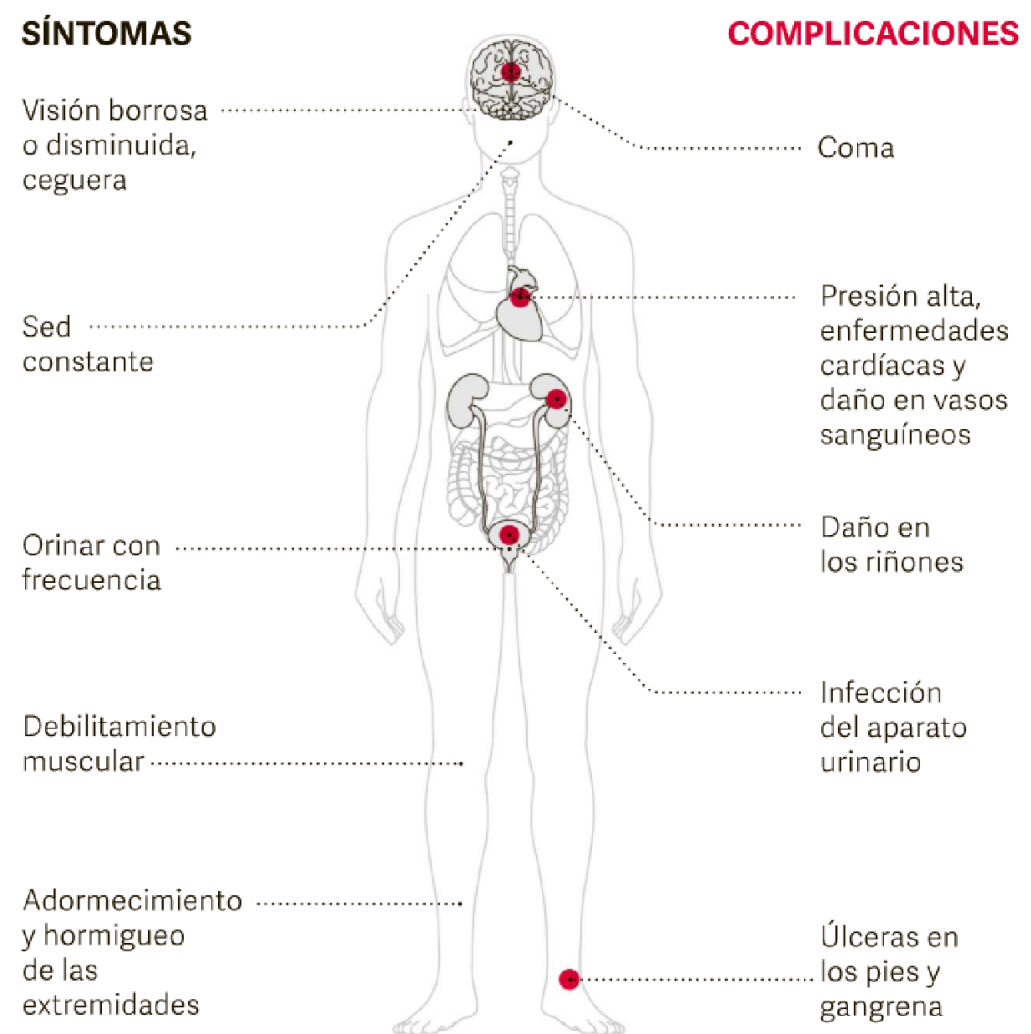

Figura 1.4: Sintomas y complicaciones de la diabetes.

para personas con diabetes, a través de la Resolución 423/2018, que da cumplimiento a las actualizaciones previstas por la ley Nacional № 26.914 de Control de Diabetes.

- En 2018 se estuvo trabajando en la implementación de la Red Nacional de Manejo del Pie Diabético, con el propósito de articular el primer nivel de atención con el segundo y el tercero para la prevención y el tratamiento de las personas con pie diabético.

\subsubsection{Complicaciones}

Diversas complicaciones a la salud pueden asociarse a la diabetes. Algunas son más directas ya que se relacionan con niveles altos de GP. Esto se puede ver reflejado en el nivel de Hemoglobina Glicosilada ( $\mathrm{HbA1c}$ ). En términos simples, se puede decir que consiste en un examen que aporta una visión retrospectiva del control de la diabetes. En efecto, indica la media de los niveles de azúcar en los últimos 3 meses. Los glóbulos rojos que circulan por la sangre contienen una proteína llamada hemoglobina. La glucosa, que también circula por la sangre, se adhiere a la hemoglobina durante un periodo de entre 90 y 120 días. De esta manera, la prueba de HbA1c se basa en la medición de la cantidad de glucosa adherida a los glóbulos rojos y su resultado se expresa en porcentaje, que determina el nivel medio de glucemia durante el trimestre anterior a la prueba. Además, desde 2010, la American Diabetes Association (ADA) estableció esta prueba como un examen de diagnóstico de la enfermedad: un resultado igual o superior a $6,5 \%$ determina que una persona tiene diabetes, mientras que uno de entre $5,7 \%$ y $6,4 \%$ se considera prediabetes [2].

En las personas con DMT1 aparecen síntomas y signos de la diabetes, como pueden ser sed excesiva, visión borrosa, aumento del apetito, pérdida inexplicable de peso, fatiga, infecciones frecuentes, necesidad de orinar a menudo, entre otros padecimientos. La DMT2 puede presentar síntomas parecidos pero más leves o a veces incluso asintomática. Aún no 


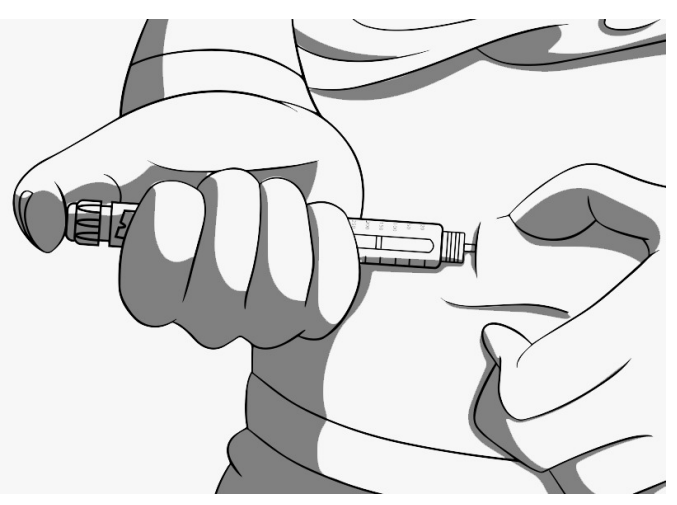

Figura 1.5: Suministro de insulina mediante inyecciones diarias. Se utiliza insulina de acción lenta para compensar los niveles basales y de acción rápida para bolos de corrección y de compensación de comidas.

se comprenden bien las causas de la misma pero hay una estrecha relación con el sobrepeso y la obesidad, el aumento de la edad y también con el historial familiar, incluyendo factores étnicos. En un caso extremo, una hiperglucemia prolongada puede llevar a la cetoacidosis diabética, debido a que sin suficiente insulina, el organismo comienza a descomponer las grasas para obtener energía lo que produce una acumulación en el torrente sanguíneo de ácidos denominados cuerpos cetónicos. En la Fig. 1.4 se detallan las diferentes afecciones que puede sufrir una persona con diabetes. En diversas publicaciones puede verse en detalle las complicaciones asociadas a la diabetes [4,8,9].

Mientras mayor sea la duración de la diabetes, mayor es el riesgo. Las complicaciones de largo término pueden dividirse en dos grandes grupos:

- Complicaciones macrovasculares: Afecciones al corazón debido a que la presión sanguínea elevada y la resistencia a la insulina aumentan el riesgo a la enfermedad coronaria. Aumenta el riesgo a un ataque cerbrovascular (ACV), incluyendo al accidente isquémico transitorio, deterioro cognitivo leve, entre otros. El estrechamiento de los vasos sanguíneos resulta en enfermedades vasculares periféricas y reduce la circulación de sangre en las extremidades. Lesiones en los pies suelen curarse lentamente, contribuyendo a gangrenas u otras afecciones.

- Complicaciones microvasculares: Presión sanguínea alta y altos niveles de GP pueden dañar los vasos sanguíneos del ojo, causando retinopatía, cataratas y glaucoma. También afecta al funcionamiento de los riñones, resultando en nefropatías y a al sistema nervioso periférico (neuropatía) lo que puede conducir dolores o falta de sensibilidad a lesiones.

\subsubsection{Tratamientos}

El registro de la diabetes puede rastrearse hasta alrededor de $1550 \mathrm{AC}$. El papiro egipcio descubierto por Georg Ebers se considera una de los tratados médicos más antiguos [10]. En el mismo se hacía referencia a personas con trastornos en la orina. Los indios y griegos también tenían registro de personas que orinaban un líquido dulce y pegajoso. El médico británico Thomas Willis fue quien acuñó el término Diabetes Mellitus, el cual deriva del griego, pudiéndose traducirse como lo que pasa a través dulce como la miel, haciendo referencia a la orina dulce que expedían cierta clase de personas.

A pesar de que se distinguía a la diabetes como un trastorno, las personas que la padecían tenían pocas posibilidades de sobrevivir. Las dietas anoréxicas promovidas sólo conseguían prolongar en unos pocos meses la vida. Los tratamientos existentes a través de los siglos en poco diferían de los propuestos casi 2000 años antes. Esto cambió a partir 


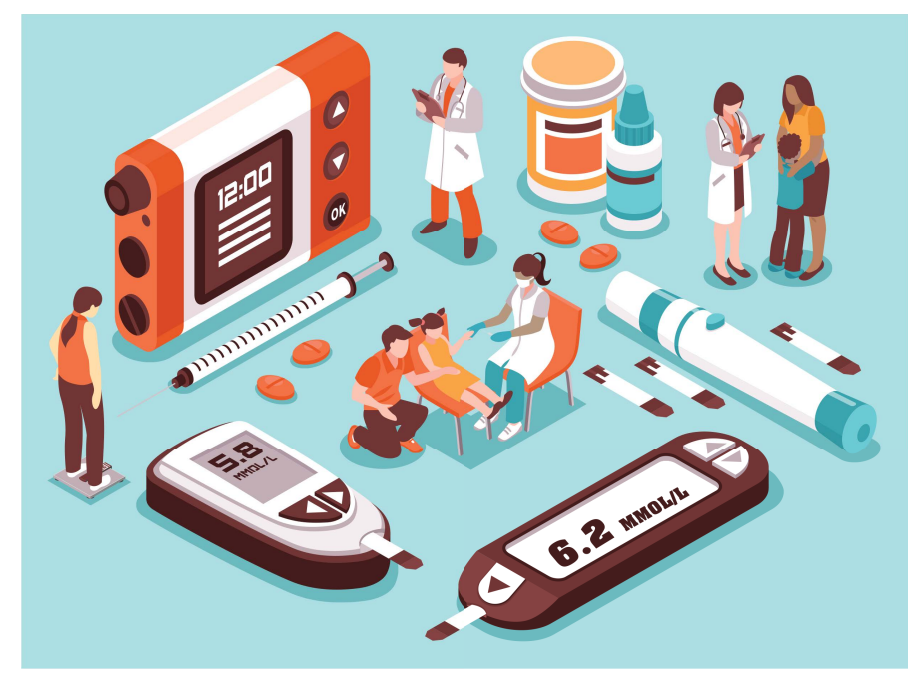

Figura 1.6: Ilustración que representa los diferentes elementos que componen al tratamiento de la diabetes, siendo estos, controles médicos, dieta, suministro de insulina, medición de glucosa, entre otros.

de la década del 20 del siglo XX. La insulina fue descubierta en el verano de 1921 por Sir Frederick Grant Banting, como consecuencia de una serie de experimentos realizados en la cátedra de John J. R. MacLeod, profesor de Fisiología de la Universidad de Toronto. El título honorífico de Sir lo alcanzaría después de obtener el Premio Nobel en medicina en 1923. Un joven de 14 años internado por una diabetes severa resultó ser la primera persona tratada con la hormona. Se acercaba una nueva era para las personas con diabetes.

La primeras insulinas disponibles provenían de origen bovino o porcino, por lo que tenían limitaciones, como las reacciones inmunológicas y su corta duración de acción. La estructura química de la insulina quedó establecida en el período 1945-1955, en una serie de investigaciones desarrolladas por Frederick Sanguer que culminaron, en 1966, con la obtención por Michael Katsoyannis de la insulina humana sintética. El espectacular desarrollo de las técnicas de ingeniería genética ha logrado la biosíntesis de la insulina por recombinación genética empleando el ADN recombinante de una cepa de Escherichia coli, síntesis conseguida en 1978, por dos grupos de científicos norteamericanos: Riggs,Itakura y col., y Goeddel, Kleid, Bolivar y col. A partir de la síntesis de la insulina, el tratamiento de la diabetes creció exponencialmente, y el desarrollo de nuevas tecnologías se expandió enormemente [11].

El tratamiento de la DM tiene como objetivo mantener los niveles de glucosa en sangre dentro de la normoglucemia o euglicemia $(\underline{\mathrm{GP}} \in[70-180] \mathrm{mg} / \mathrm{dl})$, para minimizar las complicaciones y el riesgo de la enfermedad. Principalmente se basa en la dieta, el ejercicio físico y la medicación. En la Figura 1.6 se ilustran diferentes componentes del tratamiento de la diabetes.

En una persona con DMT1 es necesaria la administración exógena de insulina ya que el páncreas es incapaz de producir esta hormona. La insulina se administra a través de inyecciones en la grasa existente debajo de la piel del brazo o el abdomen (como se ejemplifica en la Fig. 1.5), ya que si se tomase por vía oral sería destruida en el aparato digestivo antes de pasar al flujo sanguíneo. A este tratamiento se lo define como Multiples Inyecciones Diarias (Multiple Daily Injections (MDI)). Las necesidades de insulina varían en función de los alimentos que se ingieren y de la actividad física que se realiza. Las personas que siguen una dieta estable y una actividad física de forma regular varían poco sus dosis de insulina. Sin embargo, cualquier cambio en la dieta habitual o la realización de algún deporte exigen modificaciones de las pautas de insulina [1].

La insulina puede inyectarse a través de distintos dispositivos. Esta tesis se centrará 
en el tratamiento mediante bombas de insulina. Éstas consisten en dispositivos portátiles que administran insulina de acción rápida las 24 horas del día. De pequeño tamaño y no invasivas (a comparación con el suministro intravenoso), las bombas de insulina administran la insulina a través de un tubo pequeño (catéter) y una cánula (denominados equipo de infusión) que se implanta bajo la piel. La cantidad de insulina administrada se puede ajustar para satisfacer las necesidades de cada persona. A este tratamiento se lo denomina Inyección Continua de Insulina Subcutánea (Continuous Subcutaneous Insulin Infusion (CSII)).

La insulina se la caracteriza por el Tiempo de inicio, el cual transcurre antes de que la insulina llegue a la sangre y comience a bajar el nivel de glucosa; el Punto de acción máxima, que es cuando la insulina tiene su máxima potencia con respecto a la reducción de glucosa en la sangre; y la Duración, que corresponde al tiempo que la insulina continúa reduciendo el nivel de glucosa. La insulina suele medirse en Unidades Internacionales (UI o simplemente $\mathbf{U}$ ) o en concentración molar ( $\mathrm{pmol} / \mathrm{L}$ ). A su vez, existen diferentes tipos de insulina, de acuerdo a la necesidad o disposición del sujeto [12,13]:

- La insulina de acción rápida comienza a surtir efecto 15 minutos después de la inyección, tiene su máximo efecto al cabo de una hora y es eficaz durante dos a cuatro horas. Tipos: insulina glulisina (Apidra), insulina lispro (Humalog) e insulina aspart (NovoLog).

- La insulina regular o de acción breve generalmente llega al flujo sanguíneo 30 minutos después de la inyección, tiene su máximo efecto de dos a tres horas después de la inyección y es eficaz durante aproximadamente tres a seis horas. Tipos: Humulin R, Novolin R.

- La insulina de acción intermedia generalmente llega al flujo sanguíneo aproximadamente dos a cuatro horas después de la inyección, tiene su máximo efecto de cuatro a doce horas después de la inyección y es eficaz durante aproximadamente doce a dieciocho horas. Tipos: NPH (Humulin N, Novolin N)

- La insulina de acción prolongada generalmente llega a la sangre varias horas después de la inyección y tiende a mantener bajo el nivel de glucosa durante un periodo de 24 horas. Tipos: Insulina detemir (Levemir) e insulina glargina (Lantus)

La insulina puede mezclarse previamente para resultar más útil a personas con problemas de vista, de destreza manual, seguir indicaciones y otros casos. La insulina de acción rápida suele utilizarse para compensar comidas y realizar correcciones, mientras que la insulina de acción prolongada se utiliza para mantener valores basales, o realizar ciertas actividades. Mantener un control apropiado de la glucosa mediante el tratamiento MDI resulta dificultoso en comparación con CSII [14]. En el caso del tratamiento CSII se utiliza siempre insulina rápida. En cambio, el tratamiento MDI combina insulina de acción lenta para valores basales e insulina rápida para compensación de comidas o correcciones en el nivel de glucosa.

\section{Terapia Basal-Bolo}

Actualmente las personas que utilizan el tratamiento CSII deben tener presente dos aspectos: el control sobre su glucosa basal o de ayuno y la compensación de comidas o valores anormales de glucosa [12, 15. El primer aspecto se logra mediante el suministro basal. Esto consiste en pequeñas cantidades de insulina suministradas de forma continua, siguiendo un perfil diario configurado junto a un clínico. Se mide en Unidades por hora. El segundo aspecto se realiza por medio del suministro de una cierta cantidad de insulina en un instante dado, denominado bolo. Este se mide en Unidades y su acción es mucho más 

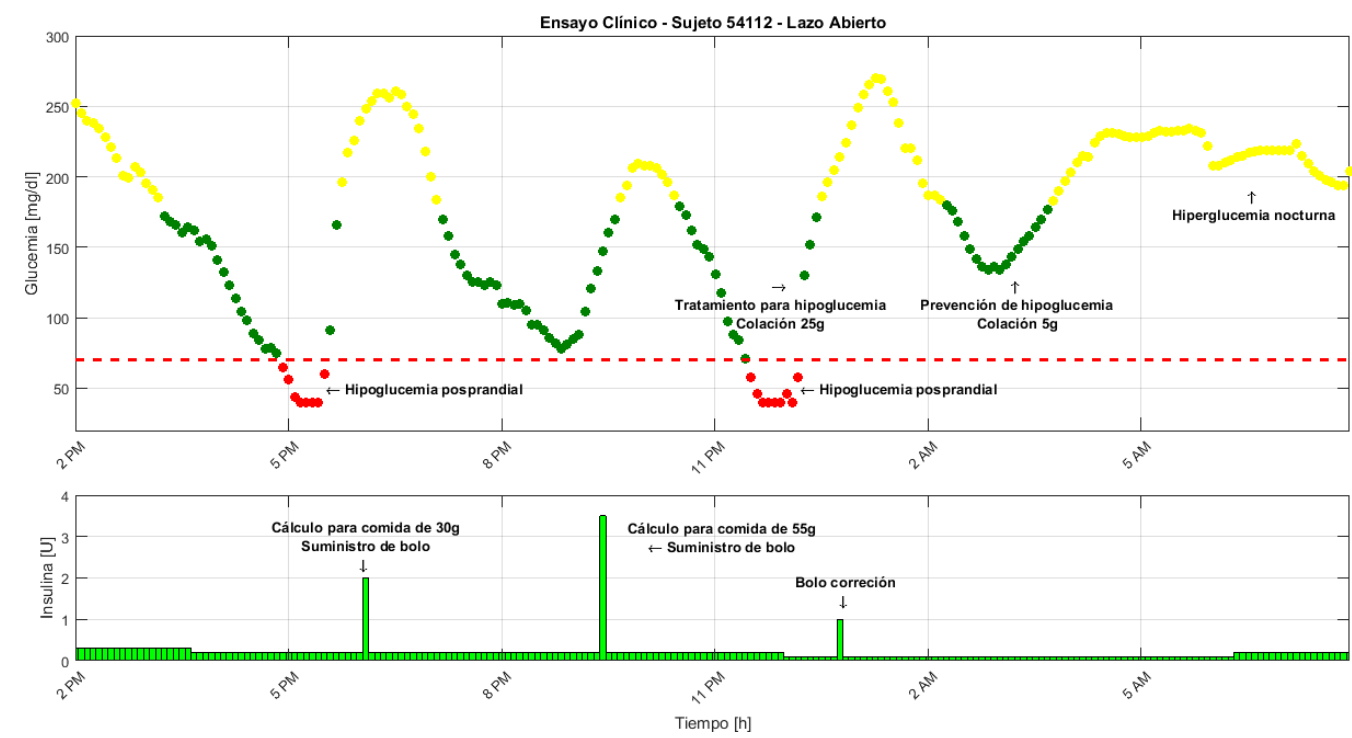

Figura 1.7: Ejemplo de tratamiento basal-bolo de un persona en su hogar mediante la terapia CSII. En la parte superior se observa el registro de CGM. Las muestras en amarillo indican hiperglucemia, en verde normoglucemia y en rojo en hipoglucemia. La línea a trazos roja indica el límite de la excursión hacia hipoglucemia $(70 \mathrm{mg} / \mathrm{dl})$. Debajo puede observarse el suministro de insulina.

agresiva, por lo que se deben tener distintas precauciones a la hora de suministrarlo, como la insulina activa en el cuerpo, la realización de actividad física, cantidad de alimentos a ingerir, entre otras variantes.

Hoy en día hay herramientas denominadas como calculadoras de bolos, lo que facilita al usuario la determinación del bolo a suministrase 16 18. Estas pueden estar incluidas en bombas de insulina, medidores de glucosa mediante un pinchazo en la yema de los dedos e incluso en aplicaciones para smartphone convencionales. No es trivial mantener un buen control a lo largo del día, por lo que las personas recurren a diversos elementos, habiendo múltiples guías, libros y referencias 14, 19]. Algunos de estas herramientas se mencionan a continuación:

Total Daily Insulin (TDI): la insulina total diaria corresponde al promedio diario de uso de insulina. Es clave para un buen control glucémico, incluso para la sintonización de controladores de lazo cerrado.

Basal Rate (BR): la tasa basal corresponde a la dosis de insulina que mantiene a la glucosa chata en un rango deseado durante el sueño y el ayuno. Suele ser la mitad de TDI y tiene un gran impacto en el control diario.

Carbohidrate Ratio (CR): el indice insulina/carbohidratos representa cuantos gramos de Carbohidratos (CHO) pueden ser cubiertos o compensados por una unidad de insulina. A veces se representa de la forma I:CHO.

Correction Factor $(\overline{\mathbf{C F}})$ : el factor de corrección o sensibilidad representa cuanta GP puede caer tras el suministro de una unidad de insulina de acción rápida.

Duration of Insulin Action (DIA): la duración de la acción de la insulina corresponde a cuanto tiempo un bolo de insulina actúa sobre la glucosa. Permite estimar la insulina que se acumula en el espacio subcutáneo (Insulin-On-Board (IOB) $)$.

Estos parámetros suelen variar a lo largo del día y deben mantenerse actualizados para un buen control glucémico. Una fuente de error frecuente se debe a la estimación de CHO de la comida ha ingerir. El cálculo de un bolo para la compensación de comidas se puede hacer mediante la siguiente ecuación por ejemplo:

$$
\text { Bolo }=\frac{C H O}{C R}+\frac{G P_{m e d}-G P_{o b j}}{C F}-I O B,
$$




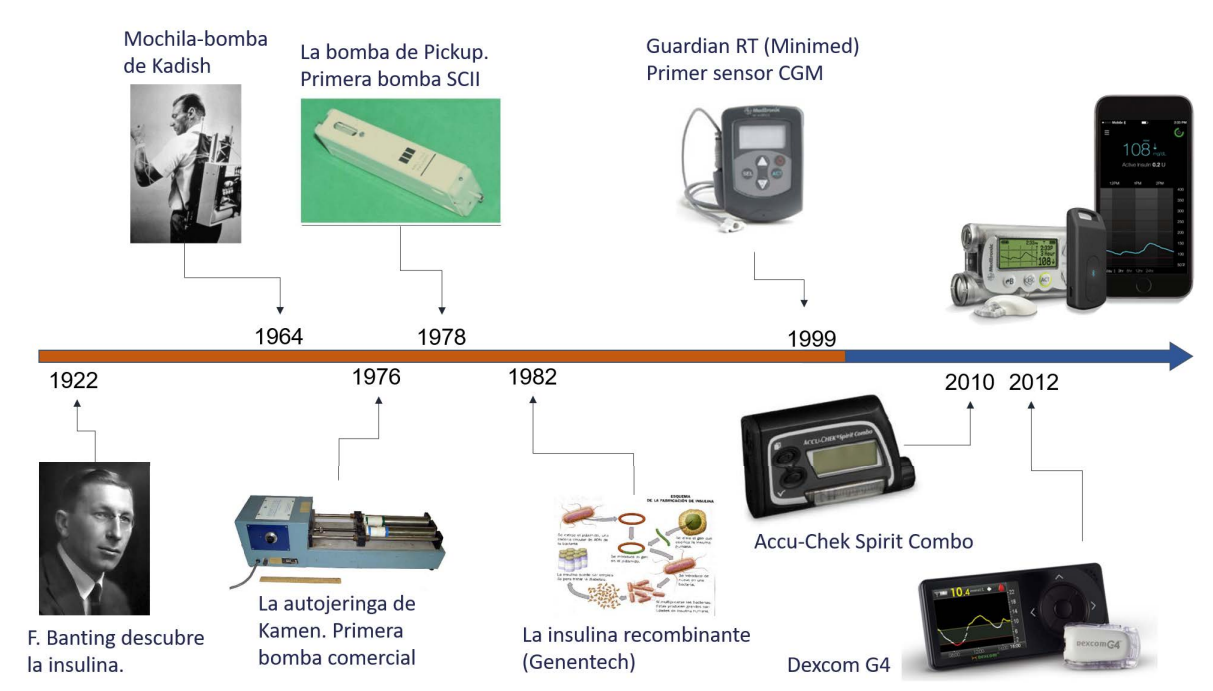

Figura 1.8: Evolución en el tiempo de la tecnología asociada a la diabetes.

donde $G P_{\text {med }}$ es la glucosa medida en el instante a suministrar el bolo y $G P_{o b j}$ es la glucosa objetivo. Estas herramientas son utilizadas tanto en terapias a lazo abierto como a lazo cerrado.

En la Fig. 1.7 se muestra la excursión glucémica de una persona con DMT1 bajo tratamiento mediante CSII. Este gráfico fue obtenido durante los ensayos clínicos realizados en el país durante el año 2017 [20] (más adelante en la tesis se referirá a esta experiencia). Consiste en el tratamiento a lazo abierto de uno de los participantes. Se pueden visualizar diferentes situaciones a las que se enfrenta la persona como pueden ser ingestas, hiperglucemias e hipoglucemias. En la parte superior se observa el registro de CGM (a una tasa de muestreo de 5 minutos), indicado mediante el color amarillo muestras en hiperglucemia, en verde muestras en normoglucemia y en rojo en hipoglucemia. La línea a trazos roja indica el límite de la excursión hacia hipoglucemia $(70 \mathrm{mg} / \mathrm{dl})$. Debajo puede observarse el suministro de insulina. Se pueden apreciar los cambios en el perfil basal a distintas horas. A su vez se visualizan 3 bolos, siendo 2 de ellos para compensación de ingestas y uno de corrección.

\subsubsection{El rol de la tecnología}

El control glucémico para personas insulino-dependientes dio un vuelco con la aparición de nuevos dispositivos tecnológicos. Estos permitieron llevar una noción de la excursión de su GP. realizar predicciones, suministrar de forma continua insulina y llevar registros minuciosos de su cotidianeidad para análisis de parte de un médico o los mismos pacientes. En la Fig. 1.8 se muestra una línea de tiempo que abarca los siglos XX y XXI, desde el descubrimiento de la insulina hasta los actuales sistemas de Páncreas Artificial [21,22]. Puede observarse los distintos dispositivos que se han ido desarrollando, los cuales se detallan en las siguientes secciones.

\section{Monitores Continuos de Glucosa}

Las personas diabéticas tienen dos formas de medir su GP mediante mediciones de sangre capilar y mediante sensores continuos de glucosa (Continuous Glucose Monitor (CGM)). Las medidas capilares Self-Monitoring of Blood Glucose (SMBG) presentan una gran variabilidad ya que dependen de la calibración de un medidor, la temperatura ambiente, humedad, tamaño y calidad de la muestra, posibilidad de substancias que interfieran, vejez de las tiras reactivas, entre otros factores [23. Por otro lado, el sistema 
CGM es mínimamente invasivo y tiene como principal ventaja de medir la concentración de glucosa intersticial en tiempo real por medio de un sensor subcutáneo [24. Algunos precisan la calibración mediante SMBG por lo menos dos veces al día, pero los recientes avances apuntan a reducir o evitar estas calibraciones. Desde el punto de vista de tratamiento, tienen la gran ventaja de mostrar perfiles de glucosa y la tendencia durante el día, en vez de sólo un valor en un instante dado. Esto permite un mejor entendimiento y control de la glucosa durante diferentes situaciones, principalmente durante el ayuno y etapas posprandiales, pero también el efecto sobre la variabilidad de la glucosa, como por ejemplo de la actividad física 25.

Reconocer hipo- o hiperglucemias mediante SMBG puede resultar muy dificultoso o incluso fallar. Los CGM permiten mejorar los tratamientos de suministro de insulina, ajustar el control glucémico y prevenir hipoglucemias. También el monitoreo continuo ha demostrado reducir la HbA1c y reducir la cantidad de episodios hipoglucemicos [25]. Aún así, los CGM presentan un alto costo, su disponibilidad es limitada en nuestro país y algunas personas presentan un grado de molestia cuando se aplican el sensor [21.

El desarrollo de los CGM se centró en empresas de origen estadounidense. El primer CGM en ser aprobado por la Food and Drugs Administration (FDA) fue el Medtronic Minimed CGM en 1999 [26]. Éste no estaba pensado para ser usado en tiempo real y estaba estipulado que debía usarse con medidas regulares de SMBG. Desde su aparición hace ya 20 años, ha mejorado sustancialmente la vida útil de los sensores, tiempos de calibración y la precisión de los CGM. Se han introducido con el paso del tiempo softwares más accesibles y amigables con el usuario, se introdujeron alarmas personalizables y la posibilidad de conexión con dispositivos móviles. Hoy en día, los CGM están aprobados para tomar decisiones sobre el tratamiento y son en algunos casos cubiertos por obras sociales y prepagas.

Un CGM subcutáneo consiste en tres elementos: un electrodo electroquímico insertado en el espacio subcutáneo que funciona como sensor de glucosa intersticial (Fig. 1.9); un transmisor, el cual hace un pre-procesamiento de la señal del sensor y envía los datos al receptor; y un receptor, el cual muestra las medidas, pueden configurarse alarmas, almacenar datos, entre otras funciones.

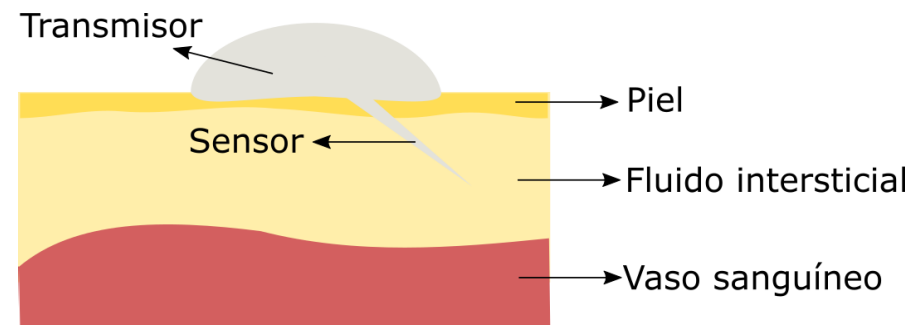

Figura 1.9: Representación esquemática del sensor subcutáneo de glucosa y del compartimento intersticial y el plasmático.

El método de sensado más común es mediante sensores electroquímicos debido al aumento en sensibilidad y precisión con el paso de los años [27]. El principio básico de funcionamiento consiste en la oxidación de la glucosa en presencia de la enzima glucosaoxidasa (GOx) que se encuentra en el electrodo. Esta presenta estabilidad ante valores variables de $\mathrm{pH}$ y temperatura. Mediante un co-sustrato, generalmente a base de oxígeno, facilita la transferencia de electrones, generando peróxido de hidrógeno $\left(\mathrm{H}_{2} \mathrm{O}_{2}\right)$ y produciendo una corriente al aplicar un potencial al electrodo. Esta corriente será proporcional a las moléculas de glucosa en el liquido intersticial. El uso de oxígeno tiene sus limitaciones, y en casos de una mala inserción o hipoxia, el sensor presentará un pobre desempeño. La corriente suministrada por el sensor luego es procesada para la estimación de la glucosa en plasma. Puede haber una diferencia en la medida de glucosa en el fluido intersticial en 
el espacio subcutáneo y una medida capilar, especialmente durante rápidos cambios de la glucemia. El retraso entre ambas medidas se considera que ronda entre los 5-15 minutos aproximadamente, pudiendo haber más variabilidad [28]. Actualmente los CGM permiten una lectura cada 5 minutos aproximadamente de forma continua y pueden llegar a reemplazar medidas mediante SMBG para correcciones o cálculo de bolos previos a las comidas. En caso de que se indique una hipoglucemia, siempre se debe constatar con una medida capilar .

La precisión de estos sistemas se reporta comúnmente mediante la diferencia relativa absoluta media (Mean Absolute Relative Difference (MARD) ) 29]. Esto se determina durante ensayos clínicos y se define como

$$
M A R D=\frac{1}{N_{r e f}} \sum_{k=1}^{N_{r e f}} \frac{\left|y_{C G M}\left(t_{k}\right)-y_{r e f}\left(t_{k}\right)\right|}{y_{r e f}\left(t_{k}\right)},
$$

donde $y_{C G M}$ es el valor medido por el CGM y $y_{r e f}$ es el valor medido de referencia en el tiempo $t_{k}$, con $k=1,2, \ldots, N_{\text {ref }}$. Aunque hay críticas a esta medida y se revisa su representatividad de la calidad del sensor debido a que está influenciado por el diseño del estudio clínico, hoy en día se utiliza para caracterizar a los CGM disponibles. Mientras menor sea el \% de MARD mejor es el desempeño del sensor. Se considera que un MARD menor al $10 \%$ es suficiente para poder realizar decisiones sobre la dosis de insulina a partir de mediciones de CGM [30].

Se pueden resaltar 4 desarrolladores de sistemas CGM disponibles en el mercado estadounidense: Abbot FreeStyle, Dexcom, Medtronic y Senseonics [21. En el cuadro 1.1 se resumen los sistemas actualmente disponibles en el momento de escribir esta tesis.

\begin{tabular}{l|cccc}
\hline & $\begin{array}{c}\text { Abott Freestyle } \\
\text { Libre }\end{array}$ & Dexcom G6 & $\begin{array}{c}\text { Medtronic Guardian } \\
\text { Sensor 3 }\end{array}$ & $\begin{array}{c}\text { Senseonics } \\
\text { Eversense }\end{array}$ \\
\hline MARD & $9.4 \%$ & $9 \%$ & $8.7 \%$ & $8.8 \%$ \\
Duración & 14 días & 10 días & 7 días & 90 días \\
Calibración & no precisa & no precisa & 2 diarias, $3-4$ recomendadas & 2 diarias \\
Tiempo de "warm up" & 1 hora & 2 horas & 2 horas & 24 horas \\
Alarmas & No & $\mathrm{Si}$ & $\mathrm{Si}$ & $\mathrm{Si}$ \\
\hline
\end{tabular}

Tabla 1.1: CGM disponibles actualmente en el mercado (E.E.U.U.) 21.

Lo monitores continuos de glucosa han mejorado el control de glucosa para las personas con DMT1 y permiten un mejor estudio y desarrollo de tratamientos. Sin embargo, hay limitaciones que deben tenerse en cuenta. En primer lugar, el costo de estos sistemas puede ser prohibitivo para muchos y muchas, aún más en países periféricos. No sólo hay un costo inicial de acceso, sino que hay costos mensuales en el reemplazo de los sensores y transmisores. En nuestro país, es muy limitado el acceso a estos dispositivos, siendo el Freestyle Libre el único accesible en el mercado. De hecho, este sensor no es considerado propiamente un CGM ya que no proporciona medidas continuas. Para acceder a las mediciones debe acercarse un dispositivo con tecnología NFC (Near Field Communication). Hoy en día existen propuestas que permiten la comunicación bluetooth entre el sensor Freestyle y un celular por medio de un dispositivo que se debe adicionar al lugar de inserción. Cabe destacar que Medtronic ofrece en el mercado nacional el sistema SAP (sensor y bomba 640G). En el país, la Ley Nacional de Diabetes № 26.914 fue sancionada en 2013 como una ampliación de la ley original de 1989. Garantiza la cobertura del $100 \%$ del tratamiento de la enfermedad por parte del sistema de salud, sea público o privado. Su reglamentación estipula que cada dos años se hará una actualización del listado de medicamentos y tecnologías cuya cobertura estaría garantizada por la propia normativa, para mantener al día la provisión de las mejores herramientas disponibles. Sin embargo, esta actualización aún no ha sucedido. Desde la Asociación Civil Diabetes Argentina (ADA), la 
Liga Argentina de Protección al Diabético (LAPDI) y la Federación Argentina de Diabetes (FAD) temen que esta tardanza se deba a una política de recorte y ajuste en detrimento de lo que garantiza el espíritu de la ley: incluir los nuevos medicamentos y tecnologías que ya son utilizados a diario por los personas con diabetes en la Argentina y en otros países del mundo y que han demostrado contribuir a mejorarles significativamente el control de su enfermedad y su calidad de vida.

A su vez, debe considerarse que se requiere un lugar de inserción del sensor diferente al de la bomba de insulina en caso de que la hubiere. No todo sitio está aprobado para la inserción, variando de acuerdo al tipo de población. También se debe tener en cuenta de que deben tener capacidad de resistir al agua y a la inmersión. La conectividad bluetooth en estos casos puede llegar a interrumpirse, perdiendo datos y posibles alarmas. Además, durante la actividad física, pueden darse rápidos cambios de glucosa, derivando a grandes retardos entre la medición del CGM y la GP.

El desarrollo de sistemas CGM sigue en auge, en busca de mejorar los tiempos de retardo y un mejor monitoreo durante la actividad física. Se busca un menor tamaño y mayor vida útil. Aunque ya muchos se utilizan en sistemas de lazo híbrido junto a bombas de insulina, sería importante que haya sistemas abiertos e independientes para el desarrollo de sistemas de Páncreas Artificial ( $(\overline{\mathrm{PA}})$, aunque los intereses económicos de las farmacéuticas y las trabas burocráticas de las instituciones reguladoras dificulten esta tarea.

\section{Bombas de insulina}

El suministro continuo de insulina (CSII) fue introducido por primera vez durante la década de 1970 para proveer flexibilidad a las personas con diabetes [15]. La bomba diseñada por el Dr. Arnold Kadish tenía el tamaño de una mochila de aproximadamente 50 litros. Luego se introdujo la "AutoSyringe", que seguía siendo grande y de difícil uso, donde las dosis de insulina se ajustaban mediante un destornillador. Ya adentrada la década de 1980 aparecieron sistemas más precisos y la compañía Animas ganó terreno a finales de los noventa, aunque hoy en día anunció su retiro en la producción de bombas de insulina. El mercado se encuentra en constante evolución, apuntando a la automatización del suministro de insulina, siendo encabezada la producción por tres grandes compañías estadounidenses: Medtronic, Insulet y Tandem. A su vez hay otras empresas en el mundo como Cellnovo, Roche Diagnostics Accu-Chek y Soil Dana Diabecare. Se estima que en el mundo más de un millón de personas usan bombas de insulina para el tratamiento de la diabetes, presentando una gran ventaja frente al tratamiento MDI, siendo este más invasivo y menos ajustable a las necesidades del control glucémico. En el cuadro 1.2 se resumen las bombas más avanzadas del mercado estadounidense 21,22. Existen diferentes tipos de bombas: la externa original, la cual es la más comúnmente usada y se hace enfoque en esta tesis; y otras, como la "patch", la cual no utiliza catéter y se conecta inalámbricamente, o la implantable, que suministra directamente en el espacio intraperitoneal.

\begin{tabular}{l|ccc}
\hline & Medtronic 670G & OmniPod DASH & Tandem X2 \\
\hline Incremento de dosis & Basal: 0.025 U/h & Basal: 0.05 U/h & Basal: 0.001 U/h \\
& Bolo: 0.025 U & Bolo: 0.5 U & Bolo: 0.01 U \\
Integración con CGM & Medtronic Guardian Sensor 3 & Dexcom G5/G6 & Dexcom G5/G6 \\
Prevención de hipoglucemia & LGS & No & Basal-IQ con G6 \\
Lazo cerrado disponible & Auto-mode & No & No \\
\hline
\end{tabular}

Tabla 1.2: Bombas de insulina disponibles en el mercado (E.E.U.U.) 21.

Las bombas de insulina consisten en un dispositivo electromecánico el cual puede suministrar insulina (generalmente de acción rápida) en el tejido subcutáneo. Están compuestas 
por un reservorio de insulina, por lo general cilíndrico, una cánula que se conecta al lugar de inserción y la bomba propiamente dicha que posee los controles, el módulo de procesamiento, un motor y las baterías. El modo de uso se lo puede caracterizar como: suministro basal, la cual permite ajustar los niveles de glucosa durante el ayuno y contrarrestar variabilidad circadiana; bolos de insulina, los cuales se utilizan generalmente durante el periodo posprandial para compensar el efecto de las comidas 31. Se pueden programar perfiles variantes dentro de las 24 horas, diferentes factores de sensibilidad, incluso la duración de la insulina para estimar la IOB, Los productores de bombas especifican una precisión de $\pm 5 \%$ de la dosis de insulina, especificada bajo métodos estandarizados internacionalmente 32,33 .

El uso de bombas no es ampliamente difundido entre las personas con diabetes, principalmente debido a su alto costo. Los médicos generalmente indican su uso para reducir altos niveles de HbA1c (mayores al $7 \%$ ), cuando hay episodios hipoglucémicos recurrentes, una amplia variabilidad glucémica o incluso pacientes motivados, ya que demanda tiempo tener maestría sobre las mismas 34 .

Hoy en día, la novedad está en las bombas definidas como Sensor-Augmented Pumps (SAP) [17. Permiten tanto a la persona como al médico optimizar la monitorización y el control de la glucosa mediante la incorporación de un sensor CGM. A su vez se desarrollaron algoritmos de suspensión de insulina cuando la glucosa es baja y se programaron métodos de prevención de hipoglucemias, denominados sistemas Low Glucose Suspend (LGS) 35, 36. Para mayor especificación de estos sistemas, el lector puede adentrarse en el capítulo 5 de esta tesis.

La principal inquietud que presentan estos dispositivos es la posible obstrucción o falla de la bomba, que puede derivar a hiperglucémias severas. Se reporta un incremento de la ketoacidosis diabética en comparación con el tratamiento mediante MDI, principalmente debido a la insulina de acción rápida y posibles fallas o obstrucciones de las bombas [37]. Otro aspecto relevante a tener en cuenta es el costo del tratamiento mediante bombas para las personas con DMT1, Los sets de infusión, adhesivos, reservas de insulina, baterías e insulina aportan un costo significativo además de la inversión inicial.

En nuestro país es limitado el acceso a las bombas de insulina. Las mismas se otorgan bajo indicación expresa y fundamentada de profesional especializado y su otorgamiento deberá ser evaluado y aprobado por la auditoría de la institución que corresponda de acuerdo a la ley N 23.753. Además, quienes realizan esas auditorías (obras sociales) son quienes luego se encargan de los gastos. Se estima que en nuestro país, alrededor de 5000 personas usan una bomba de infusión de insulina y un sensor continuo de glucosa.

\section{Impacto de la tecnología en el tratamiento de la diabetes}

Es interesante pensar y reconocer el impacto psico-social, que incluyen factores medioambientales, sociales, conductuales y emocionales, que pueden afectar a las personas con diabetes y al resultado de su tratamiento. De hecho la American Diabetes Association tomó la postura de integrar el cuidado psico-social a la atención que se le ofrece a todas las personas con diabetes y en general para optimizar los resultados sanitarios y la calidad de vida relacionada con la salud [38].

Por un lado, el uso de bombas de insulina además de ser relacionada a la imagen corporal, es asociada a un alto nivel de satisfacción, reducción de la ansiedad y mejora la autoeficacia, funcionamiento familiar y calidad de vida [39]. El uso de CGM muestra generalmente altos niveles de satisfacción y redujo el miedo a la hipoglucemia entre sus usuarios [40]. Sin embargo, un sueño más pobre y un aumento de la ansiedad han sido también reportados en padres de niños con DMT1 usando CGM [41].

Por otro lado, vivimos en un mundo de consumo masivo, donde se disponen incontables productos de diferentes formas, tamaños y aplicaciones. La tecnología para el tratamiento 
de la diabetes cae dentro de este marco. Luego de desempacar un sensor CGM y/o un set de infusión de una bomba, la persona tienen en sus manos la solución para los próximos días pero también una importante cantidad de desechos. Se considera que el $10 \%$ del peso y volumen total para el cuidado de la diabetes corresponde al producto en si, siendo el $90 \%$ restante cartón, papel envoltorio, diversos plásticos y otros componentes que idealmente irían a reciclarse. A su vez, nuevos productos para facilitar la inserción de los sensores y reducir el dolor asociado, la naturaleza estéril y desechable de los productos médicos (como lo son las tiras reactivas) y el agregado de componentes electrónicos deriva en consecuencia en pilas de materiales desechados diariamente por esta comunidad [42]. En la Figura 1.10 puede verse los desechos no reciclables debido al uso de la tecnología en el tratamiento de la diabetes. La foto fue publicada en Facebook en un grupo de tratamiento de la diabetes.

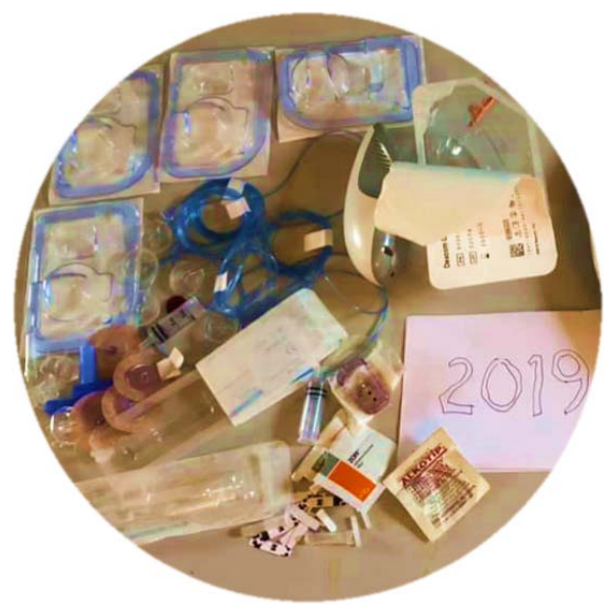

Figura 1.10: Desechos no reciclables generados en una semana por la tecnología utilizada por una persona con DMT1.

Finalmente, debe considerarse que existe un abuso por parte de las farmacéuticas hacia las personas que consumen sus productos para tratarse, y también hacia los grupos de investigación, a los cuales se les dificulta el acceso a la misma dependiendo de su lugar de origen o lineamientos. El tratamiento de la diabetes se encuentra lejos de ser democrático y de amplia difusión. En consecuencia, surgen alternativas por parte de personas con inquietud por resolver su situación a falta de respuesta de las empresas, instituciones y entes regulatorios.

\subsection{Páncreas Artificial}

La idea de automatizar el control de la GP se remonta a estudios clínicos realizados en la década de 1970 [43. El concepto de "células beta artificiales" obtiene un gran impulso cuando se desarrollan bombas de insulina vía subcutánea CSII y más tarde los monitores CGM poniendo al concepto de PA en un marco mundial de desarrollo científico y tecnológico. Gracias al impulso dado por la Juvenile Diabetes Research Foundation (JDRF) en la década del 2000 [44], diversos grupos han desarrollado a partir de la ingeniería algoritmos de control. Estos son el cerebro detrás de escena del PA, los cuales vinculan los CGM con la terapia CSII. En la Figura 1.11 se ilustra el salto tecnológico, desde la primera bomba de insulina hasta el sistema de Páncreas Artificial actual.

Desde el punto de vista de diseño, hay diversos factores a tener en cuenta, incluyendo variables fisiológicas, limitaciones del sistema, objetivos clínicos, entre otros. Como cualquier problema de ingeniería, no hay una solución absoluta sino varios enfoques, cada uno con sus ventajas y desventajas. El Proyecto de Páncreas Artificial lanzado por la JDRFen 2006 propuso un camino a seguir en el desarrollo de sistemas de PA (Fig. 1.12). Consiste 


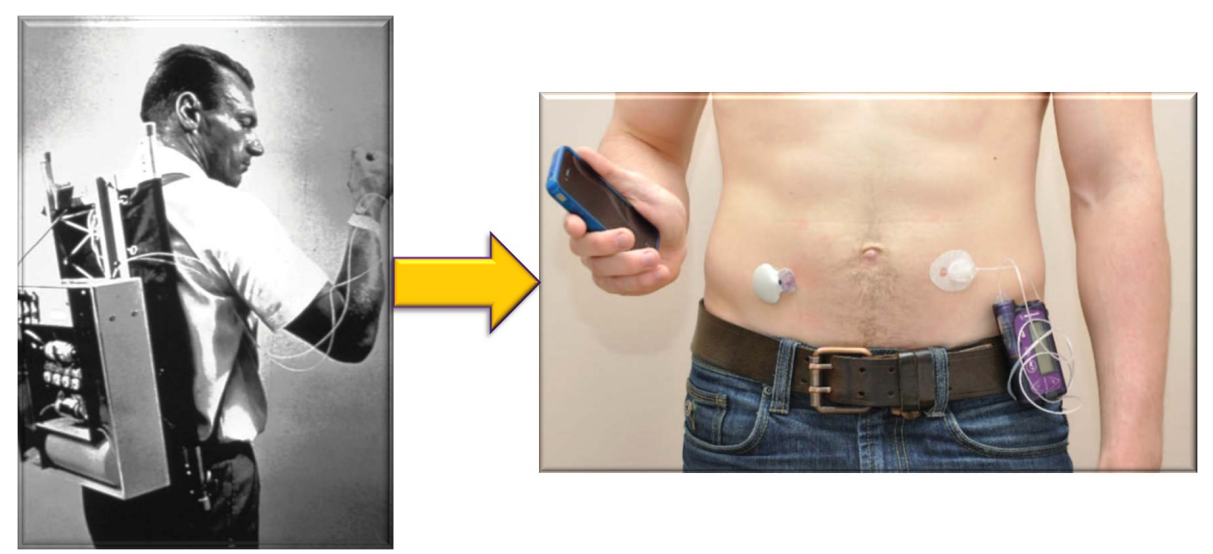

Figura 1.11: Primera bomba de insulina en 1964 por Arnold Kadish al actual diseño de la bomba comercial y sensor de glucosa subcutáneos, partes del sistema de PA portable mediante un dispositivo smartphone.

en 6 pasos graduales, donde se especifican las cualidades que tendría que cumplir el sistema, finalizando el camino en un sistema completamente automatizado. Esto permitiría aumentar drásticamente la calidad de vida de las personas con diabetes, desde niños y niñas, quienes dependen de adultos para su control glucémico, hasta adultos.

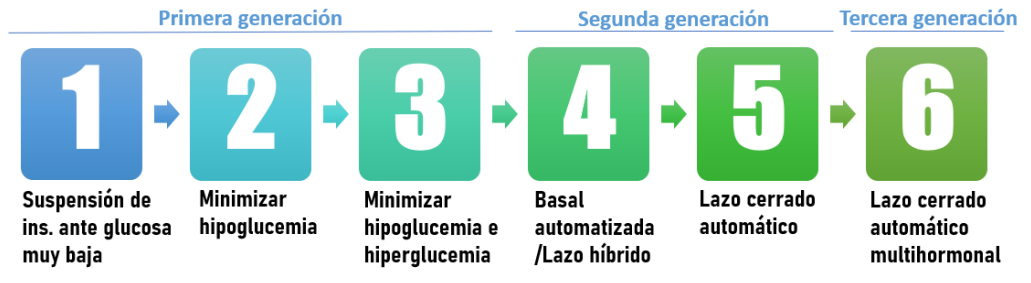

Figura 1.12: Camino de 6 pasos del sistema de Páncreas Artificial.

Los objetivos principales a elegir son maximizar el tiempo en euglicemia, minimizar los episodios hipoglucémicos, limitar la hiperglucemia posprandial o minimizar la intervención de la persona en el tratamiento. Por esta razón, la mayoría de los algoritmos propuestos tiene una estructura híbrida, donde se suministra un bolo de compensación por fuera del lazo de control 45 .

\subsubsection{Diseño de controladores}

El diseño de un controlador para este tipo de problema, más allá de su estructura teórica, depende de los objetivos elegidos a cumplir. En primer lugar, se debe considerar el valor de GP deseado. Este puede ser un valor puntual o una zona, fija o variante en el tiempo. A su vez puede contar con un modelo que le proporcione más información de las dinámicas en juego y posibles predicciones. De acuerdo a cómo se ajuste el controlador, determinará su comportamiento ante variaciones y qué tan agresiva será su respuesta [46].

El controlador proporciona una salida generalmente asociada a una dosis de insulina recomendada a suministrar mediante una bomba. Otras hormonas podrían considerarse, al igual que otras variables medidas además de la glucosa, como por ejemplo frecuencia cardíaca o un acelerómetro para detección y acción frente al ejercicio.

En las últimas 2 décadas varios tipos de algoritmos de control se han investigado, tanto 
en simulaciones como incluso en ensayos clínicos. Las estructuras más difundidas y aceptadas son el control Proporcional-Integral-Derivativo (PID), Control Predictivo basado en Modelo (MPC) y Lógica Difusa (Fuzzy logic). La señal de error entre un valor de glucosa deseado y el medido por un CGM es la entrada habitual principal de estos controladores. El clásico PID busca minimizar ese error ajustando la infusión de insulina alrededor de valores basales, y simula la acción bifásica de la insulina que suministra las células $\beta$ [47]. Mediante sus ganancias, en particular la proporcional y la derivativa, puede ajustarse la agresividad con la que responde el controlador ante variaciones en la glucosa, mientras que a la acción integral se la asocia al ajuste de valores basales, aunque en ciertos casos se la omite debido al efecto windup. Los algoritmos MPC utilizan modelos matemáticos que representan el sistema glucosa-insulina para predecir futuras concentraciones de GP a partir de las dinámicas en juego [48. No sólo buscan minimizar el error entre una dada referencia (o zona) y el valor medido sino que también dentro de un horizonte futuro se busca minimizar la acción de control y excursión de la glucosa. La gran ventaja de imponer restricciones en el diseño del controlador permite que ciertas variables fisiológicas (como la insulina disponible en el cuerpo) no excedan un cierto valor límite 49]. Finalmente, la Lógica Difusa imita y automatiza decisiones que tomaría un clínico o persona con diabetes. A partir de múltiples reglas se define el cálculo de la infusión de insulina, alejándose de formulaciones matemáticas complejas. Las variables de diseño de controladores de lazo cerrado se pueden visualizar en la Fig. 1.13. Para cada componte de la arquitectura del controlador puede haber diferentes enfoques y técnicas de diseño.

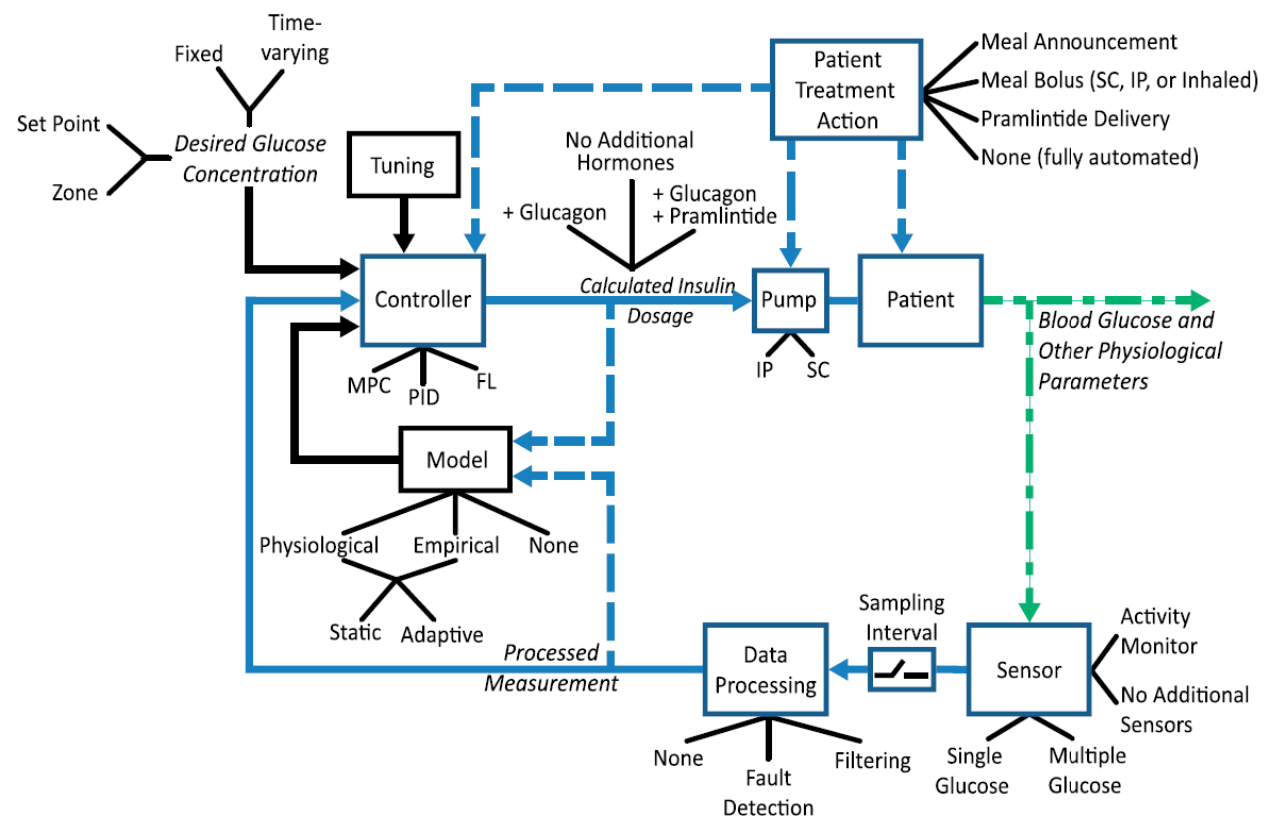

Figura 1.13: Taxonomía del diseño de sistemas de Páncreas Artificial 46.

La mayoría de los algoritmos de control incluyen módulos de seguridad para restringir el suministro de insulina, limitar la IOB, suspender la insulina cuando los niveles de GP son bajos, entre otros métodos [50]. Los parámetros individuales que se utilizan para sintonizar los controladores (detallados en 1.2.2) varían en el tiempo, por lo que algunos controladores implementan algoritmos adaptativos.

Los enfoques de los sistemas de PA se centran en estructuras tipo PID y MPC, los cuales han sido evaluados clínicamente de forma intensiva, logrando mantener un promedio de $71 \%$ del tiempo en rango a partir del 2010. La lógica difusa se encuentra en auge en el último tiempo. Hoy en día la mayoría de los grupos se centra en desarrollar capas de seguridad de prevención de hipoglucemias, basadas en sistemas LGS o limitación de 
IOB, o agregar adaptación vía métodos run-to-run. Lo que divide principalmente a los algoritmos de control es la manera en que compensan las comidas. Esto puede ser, por un lado, mediante aviso de las mismas o mediante un bolo manual, o de forma totalmente automática sin anuncio de comidas. Los algoritmos de control full closed-loop (lazo cerrado puro) son la meta final, como se define en el camino de la JDRF, los cuales van en aumento con el paso de los años. Debido a las vastas publicaciones que hacen referencia al último párrafo, el lector es referido a las siguientes revisiones [22, 45, 46, 51.

Teniendo en cuenta el aspecto comercial, lo que podría considerarse el primer sistema de PA disponible en ciertos mercados (principalmente en el estadounidense) es la bomba SAP MiniMed 670G de Medtronic [52]. Este corresponde a un sistema híbrido aprobado por la FDA que ajusta los valores de insulina basal (modo AutoMode) mediante un controlador de tipo PID, teniendo en cuenta un lazo de realimentación de insulina (Insulin Feedback (IFB) ) y predicciones de glucosa para evitar episodios hipoglucémicos (sistema LGS). Para compensar las comidas requiere del anuncio de las mismas, información de CHO, aceptación por parte del usuario de bolos recomendados, calibración del sensor, cambios manuales del set-point, estando aún alejado del un sistema completamente automatizado [53.

Aunque por el momento el único sistema de lazo cerrado híbrido disponible en el mercado es la bomba Medtronic 670G, el lanzamiento de otros sistemas se encuentra próximo. Beta Bionics trabaja en el sistema iLet el cual suministraría tanto insulina como glucagón a partir de información de CGM. Esta compañía trabaja con Senseonics Eversense CGM (implantable). Por otro lado, OmniPod está desarrollando su sistema de lazo cerrado Horizon mediante un sensor Dexcom G6 y un algoritmo MPC de Insulet. Por último, Tandem desarrolla su sistema Control-IQ mediante bombas Tandem X2, sensores Dexcom G6 y la tecnologías TypeZero (compañía de desarrollo de sistemas de PA) 21.

\subsubsection{Desafíos del control de glucosa}

La ruta subcutánea resulta hoy en día la opción más viable para los sistemas de PA debido a su carácter mínimamente invasivo y la aceptación médica de las bombas de insulina y monitores de glucosa. Sin embargo, esta ruta trae consigo una serie de dificultades. Estas dificultades tienen como factor común los retardos, tanto en la acción de control como en la medición de la variable controlada. La insulina luego de ser suministrada, debe absorberse para circular en el torrente sanguíneo. La concentración de insulina en plasma, además de aparecer en otros espacios en vez de hacerlo fisiológicamente por medio del páncreas hacia la vena porta pasando por el hígado, tarda en actuar sobre la GP. Por otro lado, el efecto sobre la glucosa tarda en manifestarse en el líquido intersticial (y no lo hace de la misma manera, especialmente cuando hay cambios drásticos) para luego ser medida [54].

La gran variabilidad del sistema a controlar es uno de los mayores desafíos. No sólo la diferencia entre los rangos etarios, sino entre cada persona en sí (cada una de ellas es un mundo), por lo que realizar un controlador universal está lejos de ser el camino a seguir. Aún así, un controlador debe enfrentarse ante las variaciones que presenta una misma persona a lo largo del día y las diversas situaciones a las que se enfrenta. El estrés, el ejercicio físico, el tipo de alimentación, entre otros, son factores fundamentales a tener en cuenta. La compensación de la variación intra-paciente es fundamental. Por otro lado, fallas en los dispositivos, desconexiones, errores de medición y de cuantización, llevan a hacer una tarea sumamente compleja la de obtener un sistema de lazo cerrado estable y confiable [55].

Para facilitar la tarea de afrontar los desafíos de control, resulta conveniente sintonizar, adaptar y ajustar los parámetros del algoritmo de control en base a los datos clínicos comúnmente manejados tanto por el médico como por el usuario. Los perfiles de insulina 
basal, los coeficientes CR, CF, TDI, DIA, peso, no sólo son utilizados para definir a los controladores, sino que también los algoritmos ayudan a ajustar a lo largo del tiempo a los mismos. En definitiva, calcular la cantidad adecuada de insulina no es una tarea simple, siendo los mayores desafíos a los que se enfrenta el algoritmo de control los siguientes:

- Con un esquema de medición subcutánea existe un retardo temporal de varios minutos entre lo que se mide y lo que ocurre en sangre, es decir, lo que se mide es la historia pasada de la glucemia. Además, a pesar de los grandes avances, las mediciones suelen tener un error significativo, por lo cual los sensores requieren calibraciones diarias (ya sea mediante hardware o software).

- Al igual que con la medición subcutánea existe un retardo temporal de varios minutos asociado a la absorción plasmática de insulina que es infundida en el espacio subcutáneo. Más aún, el pico de acción de la insulina no ocurre hasta un tiempo después de infundirse $(\approx 1 \mathrm{~h})$, y su efecto prevalece por varias horas. Esto quiere decir que las decisiones que se tomen a cada instante en relación a cuánto infundir, tienen consecuencias prolongadas en el tiempo futuro. Un efecto asociado a esto es la acumulación de insulina activa (IOB). Esto quiere decir que si no se toman en cuenta las infusiones pasadas, se puede generar una acumulación de insulina y, en consecuencia, una hipoglucemia.

- Diferentes pacientes responden de manera distinta a la misma dosis de insulina (variabilidad inter-paciente).

- El mismo paciente en diversas oportunidades puede responder de manera diferente a la misma dosis de insulina (variabilidad intra-paciente).

Un aspecto fundamental para aumentar la celeridad en el desarrollo del PA fue el desarrollo de modelos matemáticos de la dinámica glucosa-insulina. Generalmente, el diseño de un algoritmo de control está basado en un modelo matemático del proceso que se pretende controlar. Es por eso que desde la década de 1970 se han propuesto numerosos modelos de simulación que describen la dinámica de DMT1 56. Estos modelos han permitido avanzar rápidamente no sólo en el diseño de diferentes controladores, sino también en las pruebas de factibilidad in silico necesarias previo a las pruebas in vivo con pacientes reales.

Los caminos, así como las posturas a tomar, son diversos a la hora de desarrollar algoritmos de control automático de glucosa. En esta primera tesis en la temática en la UNLP se embarcó en varias de esas rutas y se contempló desde diferentes miradas para poder proponer soluciones que puedan amoldarse a la cotidianidad de muchas personas con DMT1.

\subsubsection{Ensayos clínicos}

$\mathrm{El}$ ensayo intenso de controladores de forma in-silico permite validarlos para llevarlos al ámbito clínico. La etapa de ensayo clínico es fundamental para poder llevar el concepto de Páncreas Artificial a las personas con diabetes. Estos ensayos conllevan una participación multidisciplinaria de profesionales (ingenieros, médicos, nutricionistas, enfermeros, abogados), una importante gestión de recursos a partir de inversiones privadas o estatales y una fundamental colaboración con instituciones como hospitales, entes regulatorios y otras. Diversos sistemas de $\mathrm{PA}$ se encuentran en diferentes etapas desarrollo. Se han considerado ensayos en ámbito clínico, ambulatorios incluso en el entorno hogareño sobre diferentes cohortes.

Hasta la fecha, más de 100 ensayos se han realizado en países como E.E.U.U., Reino Unido, Italia, Israel, Francia, Países Bajos, Alemania, Canadá, Austria, España, Dinamarca, Eslovenia y Argentina, siendo el primero reportado en Austria en 2006 [22, 45]. La 
mayoría utiliza la vía subcutánea tanto para la medición de glucosa como para el suministro de insulina, y otros incluso agregan al glucagón como segunda acción de control para evitar hipoglucemias.

El mayor desafío para el control automático de glucosa es el periodo posprandial, ya que por un lado de la insulina de acción rápida no tiene un perfil de acción como la secreción pancreática y la acción de control, por más de que se tenga detección de comidas, no toma acción hasta que la comida tenga impacto en las mediciones de glucosa [46]. La apreciación de los usuarios del $\mathrm{PA}$ es generalmente positiva, con una percepción de las ventajas de una regulación estable de la glucosa, la reducción de la necesidad de autocontrol, el alivio a las preocupaciones diarias y el ahorro de tiempo. Por otro lado, el sistema de PA presenta dificultades prácticas de llevar y utilizar múltiples dispositivos, acompañado de la sensación de que estos controlan la vida de uno. La fatiga ante las alarmas y la falta de confianza hacia el sistema disminuye la adherencia de ciertas personas al PA [57].

\subsubsection{Control bihormonal}

Por último, cabe destacar otro enfoque para los sistemas de PA. La mayoría del diseño de los controladores y ensayos clínicos consideran como acción de control sólo la insulina. Otros, en menor cantidad, consideran como segunda acción de control a la hormona glucagón. El glucagón aumenta la concentración de glucosa en plasma, promoviendo la gliconenolisis y gluconegenesis hepática. Para esto, debe haber previamente reservas de glicógeno en el hígado.

La acción del glucagón se utiliza principalmente para contrarrestar hipoglucemias. En principio, cuando una hipoglucemia está por ocurrir, se suspende el suministro de insulina y se suministra glucagón para elevar la concentración de GP. Aún se encuentra en discusión los efectos de suministrar esta hormona de forma exógena y de la efectividad de la misma, siendo en algunos casos insuficientes y teniendo que recurrir a carbohidratos de salvataje para evitar hipoglucemias [45].

Hay limitaciones importantes en cuanto a la implementación de este control de doble acción (sistema MISO), que van más allá del diseño de la estrategia de control. En primer lugar, se precisa el uso de 2 bombas, en principio. Avances recientes han propuesto bombas de "doble cámara", pero aún se precisan dos sets de infusión y 2 lugares de inserción. Esto es una gran desventaja ya que rotar 3 lugares de inserción en el espacio subcutáneo (contando a su vez elCGM) resulta dificultoso y no es recomendable repetir lugares debido a lipohipertrofia, que puede afectar el desempeño de la insulina en particular. En segundo lugar, la estabilidad del glucagón a temperatura ambiente es mucho más breve que la de la insulina. Esto no sólo aumenta las molestias de cambiar el reservorio con mayor frecuencia (aproximadamente una vez por día) sino que eleva los costos. En esta tesis no se abarcará el estudio y desarrollo de este enfoque de control, pero es de importancia nombrar esta línea aún en discusión 58].

\subsection{Conclusiones}

Este capítulo introductorio tiene la intención de colocar al lector en el contexto y estado del arte que abarca a la diabetes, en particular la tipo 1. Se recorrieron las diversas aristas que integran al tratamiento de la diabetes, las problemáticas subyacentes y la evolución de la tecnología. A su vez, no se quiso dejar de lado el aspecto social y el impacto regional de la temática. Los conceptos desarrollados en este capítulo son esenciales para comprender y profundizar las propuestas que se hacen en el desarrollo de esta tesis. 


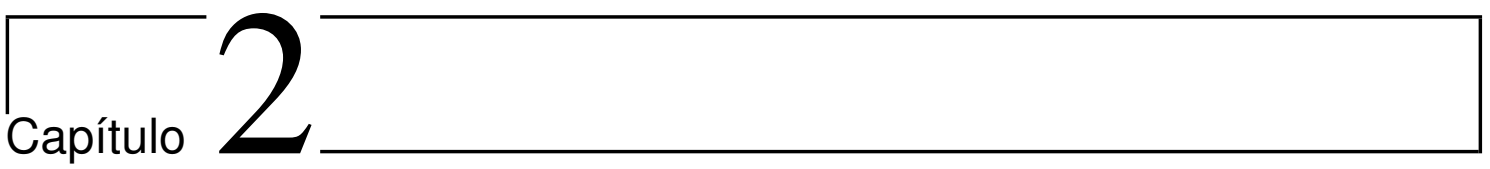

\section{Modelos y simulación in-silico}

Los modelos son una parte importante en el diseño, desarrollo y avance de sistemas de control de lazo cerrado. Un modelo es una forma de representación de un fenómeno de la "realidad" teniendo en cuenta cierto grado de aproximación. Los tipo de modelos dependen de su objetivo, pudiendo ser este: describir de forma cuantitativa relaciones en un sistema en términos de ecuaciones; interpretar y reproducir resultados experimentales; predecir la respuesta de sistemas a ciertos estímulos; o explicar cambios en observaciones o mediciones en un sistema [59]. A su vez, existen los modelos orientados al control, que se caracterizan por ser modelos paramétricos de bajo orden. Se busca que los mismos sean personalizables a partir de una pequeña cantidad de parámetros para el diseño de controladores de lazo cerrado.

Los modelos matemáticos pueden describir el comportamiento de los procesos fisiológicos en términos de ecuaciones. Estos pueden basarse en datos clínicos o en el entendimiento del proceso fisiológico. Cuando un modelo se identifica a partir de la información de entrada y salida de un proceso utilizando leyes, balances y ecuaciones ya validadas se lo denomina como método "black box." En cambio, cuando se entiende la complejidad del sistema y se toman decisiones a partir de ciertas suposiciones y simplificaciones, el método se denomina "white box." Debido a la complejidad de los sistemas fisiológicos, es muy dificultoso conocer todas las dinámicas en juego y no todos los parámetros pueden medirse. Por estas razones, los modelos matemáticos que representan procesos fisiológicos suelen realizarse mediante el método "grey box", el cual combina ambos métodos.

Para el ensayo de dispositivos médicos, los modelos cumplen un rol fundamental. Estos ayudan a entender la interacción del dispositivo con el o los procesos fisiológicos para el cual fue diseñado, permitiendo simular su respuesta ante rangos más amplios de trabajo en instancias preclínicas.

Los ensayos clínicos son esenciales para determinar el desempeño y la seguridad de estrategias de control automático de glucosa. Pero para su desarrollo, la evaluación clínica de los algoritmos es prolongada, costosa e involucra cuestiones éticas [60]. Por lo tanto, las simulaciones por computadora, gracias a la formulación de modelos matemáticos, ofrecen posibilidades para el estudio, desarrollo, prueba y validación de algoritmos de control de forma in-silico.

La dinámica glucosa-insulina ha sido extensamente estudiada y revisada por diversos autores en la literatura. Varios modelos matemáticos, basados en ecuaciones diferenciales ordinarias (ODE), han sido utilizados para simulaciones, diseño y validación de controladores para el control automático de glucosa a lazo cerrado.

Estos modelos representan a sujetos virtuales, realizados mediante modelos matemáticos compartimentales de la absorción subcutánea de la insulina, la digestión y absorción de carbohidratos, y el control de la insulina sobre la utilización y producción endógena de 
la glucosa, entre otras dinámicas, y son usados como sustitutos de personas reales para la validación in-silico de sistemas de páncreas artificial [56,61].

\subsection{Reseña de modelos}

A continuación se revisarán algunos de los principales modelos del sistema glucosainsulina propuestos en la literatura. Cabe destacar que los modelos han sido formulados para una persona sin diabetes, pero aquí se considera el suministro exógeno de insulina en lugar de la producción por parte de las células $\beta$.

\subsubsection{Modelo Sorensen}

El modelo matemático que presentó Sorensen en su tesis de doctorado en 1985 [62 representa el mecanismo fisiológico del metabolismo de la glucosa, dividiendo al cuerpo en seis compartimentos: (1) el cerebro, representando al sistema nervioso central; (2) el corazón y el pulmón, que representan los volúmenes de mezcla rápida del corazón, los pulmones y las arterias; (3) el intestino; (4) el hígado; (5) los riñones; y (6) la periferia, que incluye el músculo esquelético y tejido adiposo. Los subsistemas de glucosa, glucagón y la insulina son considerados por separado, interactuando a través de efectos metabólicos. Con el fin de obtener una representación matemática, se realiza un balance de masas en cada compartimento fisiológico.

Como consecuencia, se obtienen 12 ecuaciones diferenciales no lineales ordinarias para la dinámica de la glucosa y del glucagón (tres asociadas a variables adimensionales), siete ecuaciones lineales para la insulina y 44 parámetros. La linealidad del modelo de la insulina es debido a que se considera la DMT1, Esto no sólo induce linealidad, sino que desacopla la dinámica de la insulina de otras. Los valores de los parámetros se obtienen de la literatura y por lo tanto, sólo representan a un sujeto nominal promedio. Como todos los parámetros de este modelo son invariantes en el tiempo, el modelo no puede representar la variabilidad intra-paciente.

Este modelo ha quedado desactualizado en comparación a otros más recientes y se han encontrado inconsistencias [56]. Aún así es importante de revisar ya que fue el primero en completitud y un puntapié inicial importante para el desarrollo de futuros modelos del sistema glucosa-insulina.

\section{Variables del modelo}

- $A$ [adimensional]: estado auxiliar

- $F[\mathrm{I}, \mathrm{N}, \mathrm{L} / \mathrm{min}$, adimensional $]:$ despeje fraccional

- $G[\mathrm{mg} / \mathrm{dL}]:$ concentración de glucosa

- $I[\mathrm{mU} / \mathrm{L}]:$ concentración de insulina

- $N$ [normalizado, adimensional]: concentración de glucagón

- $Q[\mathrm{~L} / \mathrm{min}]$ : tasa de flujo de plasma vascular
- $q[\mathrm{dL} / \mathrm{min}]$ : tasa del flujo de la sangre vascular

- $T$ [min]: constante de tiempo de difusión transcapilar

- $V[\mathrm{~L}]:$ volumen

- $v[\mathrm{dL}]$ : volumen

- $\Gamma[\mathrm{mg} / \mathrm{min}$ o $\mathrm{mU} / \mathrm{min}]$ : fuente metabólica o tasa de caída

\section{Índices}




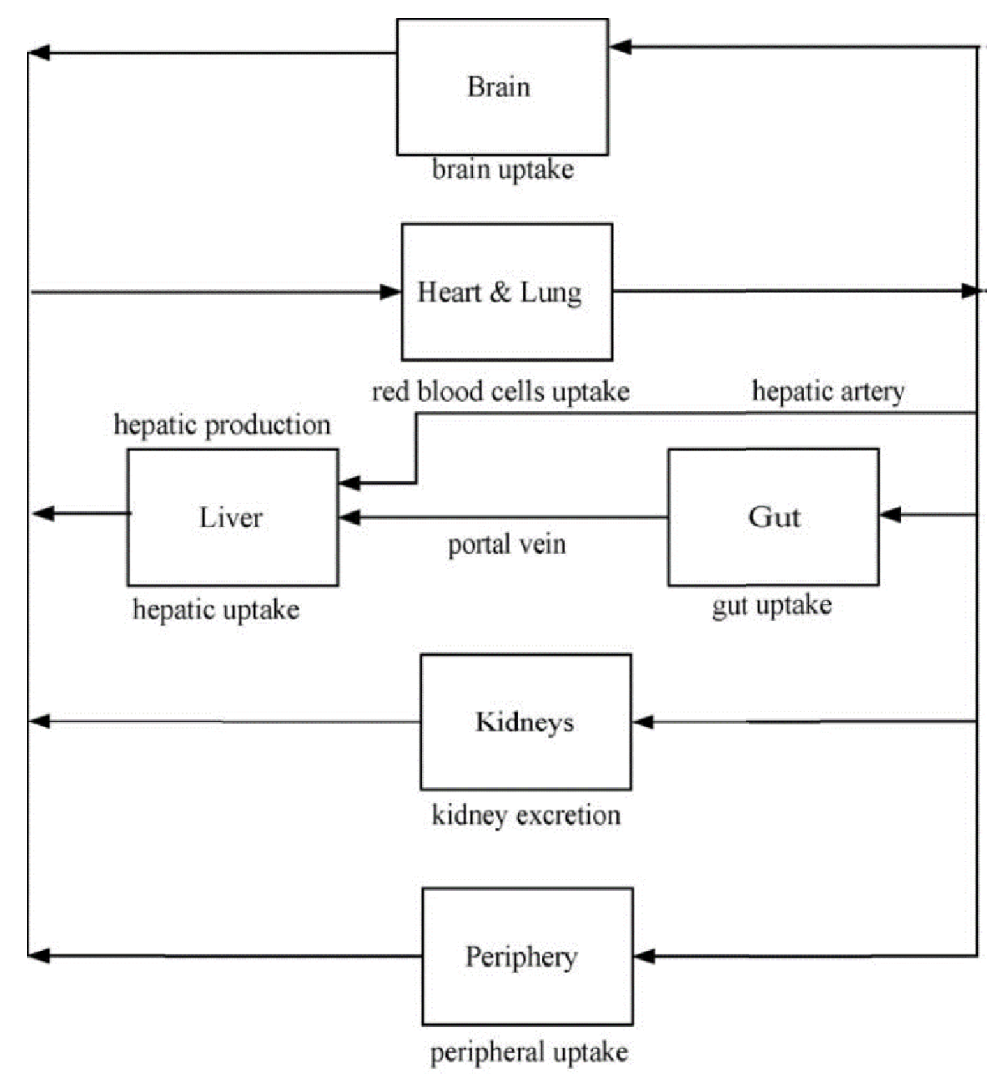

Figura 2.1: Diagrama de bloques que representa el modelo de Sorensen de 6 compartimentos.

- A: arteria hepática

- B: cerebro

- BU: absorción del cerebro

- C: espacio capilar

- G: glucosa

- H: corazón y pulmones

- HGP: producción hepática de glucosa

- HGU: absorción hepática de glucosa

- I: insulina

- IHGP: efecto de la insulina sobre HGP

- IHGU: efecto de la insulina sobre HGU
- IVI: infusión intravenosa de insulina

- K: riñón

- KC: limpieza renal

- KE: excreción renal

- L: hígado

- LC: limpieza del hígado

- N: glucagón

- NHGP: efecto del glucagón sobre HGP

- P: periferia (músculo/tejido adiposo)

- PC: limpieza periférica

- PGU: uso periférico de glucosa
- PIR: secreción de insulina pancreática

- PNC: limpieza pancreática de glucagón

- PNR: secreción pancreática de glucagón

- RBCU: uso por los glóbulos rojos

- S: estómago/intestino

- SIA: absorción insulínica hacia el flujo de sangre desde el depósito subcutáneo

- SU: toma por el estómago/intestino

- T: tejido 


\section{Dinámica de la glucosa}

$$
\begin{aligned}
& \dot{G}_{B}^{C}=\left(G_{H}^{C}-G_{B}^{C}\right) \frac{q_{B}}{v_{B}^{C}}-\left(G_{B}^{C}-G_{B}^{T}\right) \frac{v_{B}^{T}}{T_{B} v_{B}^{C}} \\
& \dot{G}_{B}^{T}=\left(G_{B}^{C}-G_{B}^{T}\right) \frac{1}{T_{B}}-\frac{\Gamma_{B U}}{v_{B}^{T}} \\
& \dot{G}_{H}^{C}=\left(G_{B}^{C} q_{B}+G_{L}^{C} q_{L}+G_{K}^{C} q_{K}+G_{P}^{C} q_{P}-G_{H}^{C} q_{H}-\Gamma_{R B C U}\right) \frac{1}{v_{H}^{C}} \\
& \dot{G}_{S}^{C}=\left(G_{H}^{C}-G_{S}^{C}\right) \frac{q_{S}}{v_{S}^{C}}+\frac{\Gamma_{m e a l}}{v_{S}^{C}}-\frac{\Gamma_{S U}}{v_{S}^{C}} \\
& \dot{G}_{L}^{C}=\left(G_{H}^{C} q_{A}+G_{S}^{C} q_{S}-G_{L}^{C} q_{L}\right) \frac{1}{v_{L}^{C}}+\frac{\Gamma_{H G P}}{v_{L}^{C}}-\frac{\Gamma_{H G U}}{v_{L}^{C}} \\
& \dot{G}_{K}^{C}=\left(G_{H}^{C}-G_{K}^{C}\right) \frac{q_{K}}{v_{K}^{C}}-\frac{\Gamma_{K E}}{v_{K}^{C}} \\
& \dot{G}_{P}^{C}=\left(G_{H}^{C}-G_{P}^{C}\right) \frac{q_{P}}{v_{P}^{C}}+\left(G_{P}^{T}-G_{P}^{C}\right) \frac{v_{P}^{T}}{T_{P}^{G} v_{P}^{C}} \\
& \dot{G}_{P}^{T}=\left(G_{P}^{C}-G_{P}^{T}\right) \frac{1}{T_{P}^{G}}-\frac{\Gamma_{P G U}}{v_{P}^{T}}
\end{aligned}
$$

\section{Dinámica de la insulina}

$$
\begin{aligned}
& \dot{I}_{B}^{C}=\left(I_{H}^{C}-I_{B}^{C}\right) \frac{Q_{B}}{V_{B}^{C}} \\
& \dot{I}_{H}^{C}=\left(I_{B}^{C} Q_{B}+I_{L}^{C} Q_{L}+I_{K}^{C} Q_{K}+I_{P}^{C} Q_{P}-I_{H}^{C} Q_{H}+\Gamma_{I V I}\right) \frac{1}{V_{H}^{C}} \\
& \dot{I}_{S}^{C}=\left(I_{H}^{C}-I_{S}^{C}\right) \frac{Q_{S}}{V_{S}^{C}} \\
& \dot{I}_{L}^{C}=\left(I_{H}^{C} Q_{A}+I_{S}^{C} Q_{S}-I_{L}^{C} Q_{L}\right) \frac{1}{V_{L}^{C}}+\frac{\Gamma_{P I R}}{V_{L}^{C}}-\frac{\Gamma_{L C}}{V_{L}^{C}} \\
& \dot{I}_{K}^{C}=\left(I_{H}^{C}-I_{K}^{C}\right) \frac{Q_{K}}{V_{K}^{C}}-\frac{\Gamma_{K C}}{V_{K}^{C}} \\
& \dot{I}_{P}^{C}=\left(I_{H}^{C}-I_{P}^{C}\right) \frac{Q_{P}}{V_{P}^{C}}+\left(I_{P}^{T}-I_{P}^{C}\right) \frac{V_{P}^{T}}{T_{P}^{I} V_{P}^{C}} \\
& \dot{I}_{P}^{T}=\left(I_{P}^{C}-I_{P}^{T}\right) \frac{1}{T_{P}^{I}}+\frac{\Gamma_{S I A}}{V_{P}^{T}}-\frac{\Gamma_{P C}}{V_{P}^{T}}
\end{aligned}
$$

\section{Glucagón}

$$
\dot{N}=\left(\Gamma_{P N R}-N\right) \frac{F_{P N C}}{V_{N}}
$$




\section{Ecuaciones auxiliares}

$$
\begin{aligned}
& \dot{A}_{I H G P}=\frac{1}{25}\left\{1,2088-1,138 \tanh \left[1,1669\left(\frac{I_{L}^{C}}{21,43}-0,8885\right)\right]-A_{I H G P}\right\} \\
& \dot{A}_{N H G P}=\frac{1}{65}\left[\frac{2,7 \tanh (0,388 N-1)}{2}-A-N H G P\right] \\
& \dot{A}_{I H G U}=\frac{1}{25}\left[2 \tanh \left(0,549 \frac{I_{L}^{C}}{21,43}\right)-A_{I H G U}\right]
\end{aligned}
$$

\section{Parámetros $\Gamma_{i}$}

Los parámetros $\Gamma_{i}$ que aparecen son los siguientes: $\Gamma_{B U}=70, \Gamma_{R B C U}=10, \Gamma_{S U}=20$, $\Gamma_{P I R}=0, \Gamma_{L C}=F_{L C}\left(I_{H}^{C} Q_{A}+I_{S}^{C} Q_{S}+\Gamma_{P I R}\right) \mathrm{y}$

$$
\begin{aligned}
& \Gamma_{H G U}= 20 A_{I H G U}\left\{5,6648+5,6589 \tanh \left[2,4375\left(\frac{G_{L}^{C}}{101}\right)\right]\right\} \\
& \Gamma_{H G P}= 155 A_{I H G P}\left[2,7 \tanh (0,388 N)-A_{N H G P}\right] \times \\
&\left\{1,425-1,406 \tanh \left[0,1699\left(\frac{G_{L}^{C}}{101}-0,4969\right)\right]\right\} \\
& \Gamma_{P G U}= \frac{35 G_{P}^{T}}{86,81}\left\{7,035+6,5163 \tanh \left[0,33827\left(\frac{I_{P}^{T}}{5,304}-5,82113\right)\right]\right\} \\
& \Gamma_{P N R}=\left\{1,3102-0,61016 \tanh \left[1,5071\left(\frac{I_{H}^{C}}{15,15}-0,46981\right)\right]\right\} \times \\
&\left\{2,9285-2,095 \tanh \left[4,18\left(\frac{G_{H}^{C}}{91,89}-0,36191\right)\right]\right\} \\
& \Gamma_{P C}=\left(1-F_{P C}^{T}\right) /\left(Q_{P} F_{P C}\right)-T_{P}^{I} / V_{P}^{T} \\
& \Gamma_{K C}= F_{K C} I_{H}^{C} Q_{K} \\
& \Gamma_{K E}= \begin{cases}71\left\{1+\tanh \left[0,11\left(G_{K}^{C}-460\right)\right]\right\} & \text { si } G_{K}^{C}<460 \mathrm{mg} / \mathrm{dl} \\
0,872 G_{K}^{C}-330 & \text { si } G_{K}^{C} \geq 460 \mathrm{mg} / \mathrm{dl}\end{cases}
\end{aligned}
$$

El modelo de Sorensen fue adaptado para representar el sistema metabólico del Minipig de Göttingen y fue parametrizado a partir de datos de ensayos clínicos con animales [63]. Este modelo consiste en 16 estados que describen 9 compartimentos diferentes. Debido a la similitud entre los sistemas metabólicos de los humanos y los cerdos, los resultados obtenidos con este modelo pueden ser migrados al sistema de metabolismo de glucosa humano. Como principal característica, posee un representación de la Sensibilidad Insulínica (SI) mediante un parámetro lineal.

Este animal fue criado genéticamente en la Universidad de Göttingen, Alemania, y se puso a disposición de la comunidad de investigación biomédica de Alemania a finales de la década de 1960. El estado de salud definido microbiológicamente y las muchas similitudes con los seres humanos hacen del Minipig de Göttingen un modelo animal ideal en todo tipo de estudios biomédicos. 


\subsubsection{Modelo Bergman}

El modelo minima $\rrbracket^{1}$ presentado por Bergman y colegas [64,65] se basa en tres factores que cumplen un rol importante en la disposición de la glucosa, siendo estos:

- Sensibilidad insulínica: la capacidad de la insulina de incrementar la disposición de glucosa en los músculos, hígado y tejido adiposo

- Efectividad de la glucosa: la habilidad de la glucosa para mejorar su propia disposición a niveles basales de insulina

- Respuesta pancreática: la habilidad de las células $\beta$ de segregar insulina ante el estímulo de la glucosa

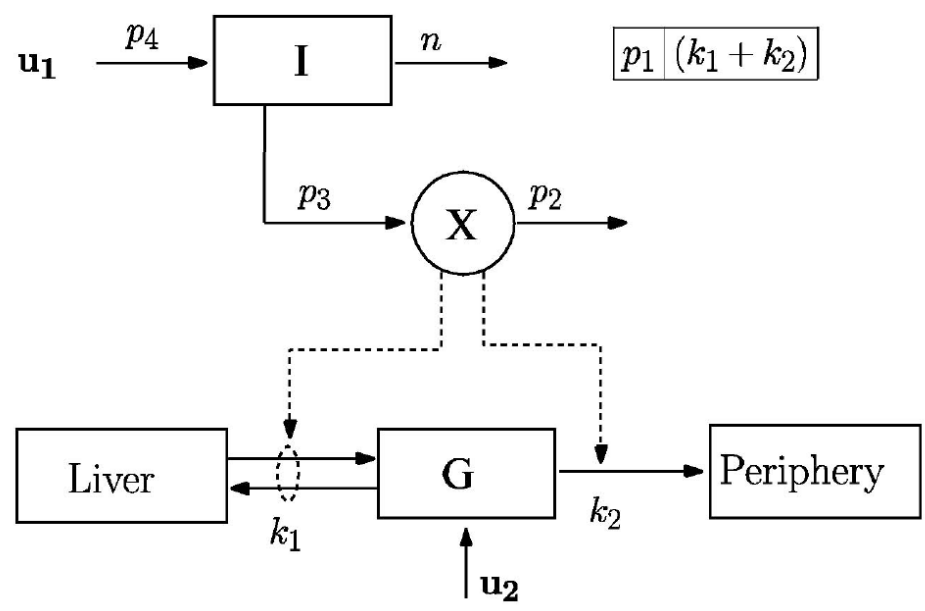

Figura 2.2: Diagrama de bloques que representa el modelo minimal de Bergman 66.

El modelo está basado en un examen IVGT (Intravenous Glucose Tolerance), el cual consiste en administrar una dosis de glucosa (generalmente 0,3 gramos de glucosa por $\mathrm{kg}$ de peso corporal) de forma intravenosa durante un periodo de 60 segundos a sujetos en ayunas desde la noche a la mañana, y luego se muestrean las concentraciones en plasma de glucosa e insulina durante un periodo de 180 minutos. Las dinámicas en el modelo son descriptas por dos componentes, donde los parámetros fueron estimados por separado dentro de cada uno (ver Fig. 2.2).

\section{Variables}

- $G(t)[\mathrm{mg} / \mathrm{gl}]$ : concentración de glucosa en plasma

- $I(t)[\mu \mathrm{U} / \mathrm{ml}]$ : concentración de insulina en plasma

- $X(t)\left[\mathrm{min}^{-1}\right]$ : efecto de la insulina en la desaparición de la glucosa

- $u_{1}(t)[\mathrm{mU} / \mathrm{min}]:$ suministro exógeno de insulina

- $u_{2}(t)[\mathrm{mg}]:$ suministro exógeno de glucosa

\footnotetext{
${ }^{1}$ Se lo suele referir a este modelo como "minimal" debido a su orden reducido. Aún así, se lo considera representativo de la dinámica glucosa-insulina y es frecuentemente utilizado tanto para la simulación como para el desarrollo de controladores.
} 


\section{Parámetros}

- $G_{b}[\mathrm{mg} / \mathrm{dl}]:$ nivel basal de glucosa

- $I_{b}[\mu \mathrm{U} / \mathrm{ml}]:$ nivel basal de insulina

- $p_{1}\left[\mathrm{~min}^{-1}\right]$ : tasa constante del consumo insulina-independiente de glucosa de los músculos, hígado y tejido adiposo

- $p_{2}\left[\mathrm{~min}^{-1}\right]$ : tasa de disminución de la capacidad de captación de glucosa de los tejidos

- $p_{3}\left[\min ^{-2}(\mu \mathrm{U} / \mathrm{ml})^{-1}\right]:$ incremento de la capacidad de consumo insulina-dependiente de glucosa en los tejido por unidad de concentración por encima de $I_{b}$

- $p_{4}\left[\mathrm{ml}^{-1}\right]$ : tasa de absorción de insulina exógena

- $n\left[\mathrm{~min}^{-1}\right]$ : tasa de descomposición de primer grado para la insulina en plasma

- $\operatorname{Vol}_{G}[\mathrm{dl}]:$ espacio de distribución de glucosa

\section{Dinámica del sistema}

$$
\begin{aligned}
\dot{I}(t) & =-n I(t)+p_{4} u_{1}(t) & I(0) & =I_{b}=\frac{p_{4}}{n} u_{1 b} \\
\dot{X}(t) & =-p_{2} X(t)+p_{3}\left(I(t)-I_{b}\right) & X(0) & =0 \\
\dot{G}(t) & =-p_{1}\left(G(t)-G_{b}(t)-X(t) G(t)+\frac{u_{2}(t)}{V o l_{G}}\right. & G(0) & =G_{b}
\end{aligned}
$$

Este modelo es ampliamente utilizado para el diseño de controladores, ya que permite obtener modelos orientados al control de bajo orden [67]. A su vez, ha sido expandido teniendo en cuenta la tasa de aparición de glucosa, ejercicio y suministro subcutáneo de insulina por otros autores para realizar predicciones de la evolución de la glucosa 66]. Cabe destacar también el modelo presentado por Magdelaine et al. [68] que tiene relevancia a la hora de desarrollar controladores MPC, el cual deriva del modelo minimal del sistema glucosa-insulina.

\subsubsection{Modelo Cambridge}

El modelo presentado por el grupo de investigación encabezado por el Prof. Hovorka de la Universidad de Cambridge consiste en un modelo compartimental de la cinética de la glucosa y la acción de la insulina 61,69. Incluye dos submodelos compartimentales de la cinética de la insulina y glucosa subcutánea, así como otro submodelo de dos compartimentos para representar la absorción de la glucosa en el tracto gastrointestinal. Una importante propiedad de este modelo es su capacidad para representar la variabilidad intra- e inter-paciente.

La variabilidad inter-paciente es representada por una población de 18 sujetos virtuales con DMT1, donde los parámetros se obtuvieron a partir de estudios clínicos en pacientes con dicho trastorno o a partir de distribuciones de probabilidad poblacionales. La variabilidad intra-paciente se llevó a cabo mediante la superposición de oscilaciones sinusoidales en un subconjunto de parámetros del modelo. Un punto débil del sujeto virtual de este modelo, es la simple representación de la absorción del intestino, además de que se podría mejorar significativamente la variabilidad intra-paciente. El modelo de simulación de Hovorka [70] fue utilizado en el desarrollo de un controlador de glucosa para niños y adolescentes con DMT1 en un proyecto de la JDRF [71, y sigue siendo utilizado por una gran parte de investigadores del área. 


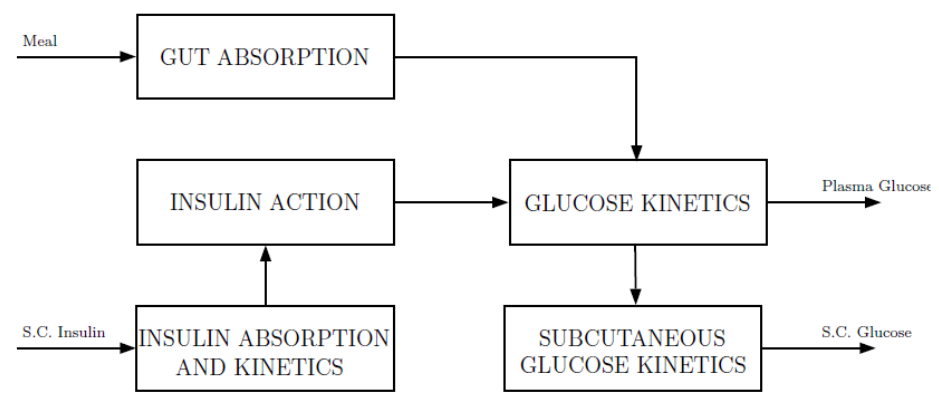

Figura 2.3: Diagrama de bloques que representa el modelo del grupo de Cambridge 72 .

\section{Acción de la insulina}

\section{Variables}

- $x_{1}(t), x_{2}(t)$ y $x_{3}(t)$ : son el efecto remoto de la insulina sobre la distribución, disposición y producción endógena de la glucosa

- $I(t)$ : concentración de insulina en plasma

\section{Parámetros}

- $k_{a i}$ : tasa de activación

- $k_{b i}$ : tasa de desactivación

- $S_{I T}=\frac{k_{a 1}}{K_{b 1}}, S_{I D}=\frac{k_{a 2}}{k_{b 2}}$ y $S_{I E}=\frac{k_{a 3}}{k_{b 3}}$ : sensibilidades de la insulina para el trasporte, distribución y la producción de glucosa

\section{Ecuaciones}

$$
\begin{aligned}
& \dot{x}_{1}(t)=-k_{a 1} x_{1}(t)+S_{I T} k_{b 1} I(t) \\
& \dot{x}_{2}(t)=-k_{a 2} x_{1}(t)+S_{I D} k_{b 2} I(t) \\
& \dot{x}_{3}(t)=-k_{a 3} x_{1}(t)+S_{I E} k_{b 3} I(t)
\end{aligned}
$$

\section{Glucosa}

\section{Variables}

- $Q_{1}(t)$ y $Q_{2}(t)$ : masas de glucosa en el compartimento accesible y no accesible

- $G(t)$ : concentración de glucosa en plasma

- EGP: producción endógena de glucosa

- $F_{01}^{C}$ : flujo de glucosa no dependiente de la insulina

- $F_{R}$ : limpieza renal del glucosa

\section{Parámetros}

- $k_{12}$ : tasa de transferencia del compartimento accesible al inaccesible

- $V_{G}$ : representa la distribución de glucosa en el compartimento accesible

- $R_{t h r}$ : umbral de glucosa para la limpieza renal 


\section{Ecuaciones}

$$
\begin{aligned}
\dot{Q}_{1}(t) & =-\left[\frac{F_{01}^{C}}{V_{G} G(t)}+x_{1}(t)\right] Q_{1}(t)+k_{12} Q_{2}(t)-F_{R}+E G P(t)+U_{G}(t) \\
\dot{Q}_{2}(t)=x_{1}(t) Q_{1}(t)-\left[k_{12}+x_{2}(t)\right] Q_{2}(t) & \\
G(t)=\frac{Q_{1}(t)}{V_{G}} & \\
E G P(t) & = \begin{cases}E G P_{0}\left[1-x_{3}(t)\right] & E G P \geq 0 \\
0 & E G P<0\end{cases} \\
F_{01}^{C} & =\frac{F_{01}^{S} G}{G+1} \\
F_{R}(t) & = \begin{cases}R_{c l}\left(G-R_{t h r}\right) V_{G} & G \geq R_{t h r} \\
0 & G<R_{t h r}\end{cases}
\end{aligned}
$$

\section{Cinética y absorción de la insulina subcutánea}

\section{Variables}

- $S_{1}(t)$ y $S_{2}$ : masa de insulina en el compartimento accesible y no accesible

- $u(t)$ : bolo administrado de insulina

- $I(t)$ : concentración de insulina en plasma

\section{Parámetros}

- $k_{a}$ : tasa de absorción de insulina

- $k_{e}$ : tasa de eliminación parcial del plasma

- $V_{I}$ : volumen de distribución de la insulina

\section{Ecuaciones}

$$
\begin{aligned}
\dot{S}_{1}(t) & =u(t)-k_{a} S_{1}(t) \\
\dot{S}_{2}(t) & =k_{a} S_{1}(t)-k_{a} S_{2}(t) \\
I(t) & =\frac{K_{a} S_{2}(t)}{V_{I}}-k_{e} I(t)
\end{aligned}
$$

\section{Absorción del intestino}

\section{Variables}

- $G_{1}(t)$ y $G_{2}(t)$ : masa de glucosa en el compartimento accesible y no accesible

- $D(t)$ : cantidad de carbohidratos consumidos en el tiempo $t$

- $U_{G}(t)$ : tasa de absorción del intestino

- $t_{\max }$ : tiempo máximo de tasa de aparición de glucosa en el compartimento accesible 


\section{Parámetros}

- Bio: biodisponibilidad de los carbohidratos de la comida

- $U_{\text {G_ceil }}$ : máximo flujo de glucosa desde el intestino derivado de $U \sim[0,02 ; 0,035]$

\section{Ecuaciones}

$$
\begin{gathered}
\dot{G}_{1}(t)=-\frac{G 1(t)}{t_{\max }}+\text { Bio } \cdot D(t) \quad G_{1}(0)=0 \\
\dot{G}_{2}(t)=\frac{G_{1}(t)}{t_{\max }}-\frac{G_{2}(t)}{t_{\max }} \\
U_{G}=\frac{G_{2}(t)}{t_{\text {max }}} \\
t_{\text {max }}= \begin{cases}t_{\text {max_ceil }} & U_{G}>U_{G_{-} \text {ceil }} \\
T_{\text {max }} & U_{G} \leq U_{G_{-} \text {ceil }}\end{cases}
\end{gathered}
$$

\section{Glucosa intersticial}

\section{Variables}

- $C(t)$ : concentración de glucosa en el tejido subcutáneo

\section{Parámetros}

- $k_{a_{-} i n t}:$ tasa de transferencia

\section{Ecuaciones}

$$
\dot{C}(t)=k_{a \_i n t}\left(G_{C}\right)(t)
$$

\subsubsection{Modelo UVA/Padova}

El grupo del Prof. Cobelli en Padova, Italia, desarrolló un modelo de simulación de comida del sistema glucosa-insulina utilizando datos tomados de 204 sujetos normales que se sometieron a un protocolo con trazadores de glucosa [73]. Esto permitió calcular los flujos de glucosa e insulina durante una comida. El modelo consta de dos subsistemas principales, descritos por dos compartimentos cada uno, siendo éstos los subsistemas glucosa e insulina. Además los acompañan varios modelos de procesos unitarios, identificados a partir de información promedio utilizando la estrategia de función forzada. Se estimaron 35 parámetros de un sujeto normal. La misma estrategia se aplicó a una base de datos más pequeña que contiene 14 sujetos con diabetes tipo II y se estimó el mismo número de parámetros que en un paciente sano.

Este modelo se ha empleado para simular un día típico con tres comidas de un sujeto normal, teniéndose en cuenta la variación de la sensibilidad insulínica y la respuesta de las células $\beta$. Una de las mayores novedades de este modelo es una descripción más detallada del tránsito de la glucosa a través del tracto gastrointestinal, pero posee como debilidad el hecho que las variaciones diurnas de determinados parámetros no han sido modeladas. A pesar de que el modelo original fue identificado usando información de sujetos normales, se utiliza para simular diabéticos tipo I sustituyendo la secreción de insulina por un modelo 
de la cinética de la insulina subcutánea. Para considerar el mayor nivel de glucosa basal en la diabetes tipo I, se aumentó la producción endógena de glucosa.

A partir de este modelo se desarrolló el simulador UVA/Padova, el cual fue aprobado por la $\mathrm{FDA}$ como sustituto en pruebas animales para ensayos preclínicos de tratamientos tanto de lazo abierto como de lazo cerrado. Esto fue de gran ayuda para los algoritmos de PA emergentes en la última década. El simulador ha sido utilizado por más de 20 grupos de investigación diferentes, lo que ha dado lugar publicaciones en revistas revisadas por pares. El simulador original se diseñó en 2008 [73,74] y se actualizó en 2013 para tener en cuenta las no linealidades de la acción de la insulina y la cinética del glucagón en la dinámica de la glucosa en rango hipoglucémico 75 . El simulador incluso fue validado en un estudio clínico [76].

Sin embargo, la falta de la representación de la variabilidad de la SI] en un sujeto se la ha señalado como una limitación significativa. El grupo de Cobelli a abordado esta limitación y han publicado análisis de características adicionales que incorporan la variabilidad diurna de la absorción de la glucosa y la sensibilidad a la insulina, lo que permite realizar una simulación más factible de duraciones de hasta un mes $77-79$. A continuación se presenta el modelo publicado en [75], el cual sigue siendo el modelo base sobre el cual se agregan variabilidad a sus parámetros.

\section{Glucosa}

Modelo de dos compartimentos (glucosa en plasma y en tejido adiposo) que describe la cinética de la glucosa.

\section{Variables}

- $G_{p}[\mathrm{mg} / \mathrm{kg}]$ : masa de glucosa en plasma y en tejido de equilibrio rápido

- $G_{t}[\mathrm{mg} / \mathrm{kg}]:$ masa de glucosa en tejido de equilibrio lento

- $G[\mathrm{mg} / \mathrm{dl}]$ : concentración de glucosa en plasma

- EGP $[\mathrm{mg} / \mathrm{kg} / \mathrm{min}]:$ producción endógena de glucosa (hígado)

- $R a[\mathrm{mg} / \mathrm{kg} / \mathrm{min}]:$ índice de aparición de glucosa en plasma

- $E[\mathrm{mg} / \mathrm{kg} / \mathrm{min}]:$ excreción renal

- $U_{i i}[\mathrm{mg} / \mathrm{kg} / \mathrm{min}]:$ utilización de glucosa independiente de la insulina

- $U_{i d}[\mathrm{mg} / \mathrm{kg} / \mathrm{min}]:$ utilización de glucosa dependiente de la insulina

(el sufijo b indica estado basal)

\section{Parámetros}

- $V g[\mathrm{dl} / \mathrm{kg}]$ : volumen de distribución de la glucosa

- $k_{1}, k_{2}\left[\mathrm{~min}^{-1}\right]$ : parámetros de velocidad de la cinética de la glucosa

\section{Ecuaciones}

$$
\begin{cases}\dot{G}_{p}(t)=E G P(t)+R a(t)-U_{i i}(t)-E(t)-k_{1} \cdot G_{p}(t)+k_{2} \cdot G_{t}(t) & G_{p}(0)=G_{p b} \\ \dot{G}_{t}(t)=-U_{i d}(t)+k_{1} \cdot G_{p}(t)-k_{2} \cdot G_{t}(t) & G_{t}(0)=G_{t b} \\ G(t)=\frac{G_{p}}{V_{G}} & G(0)=G_{b}\end{cases}
$$




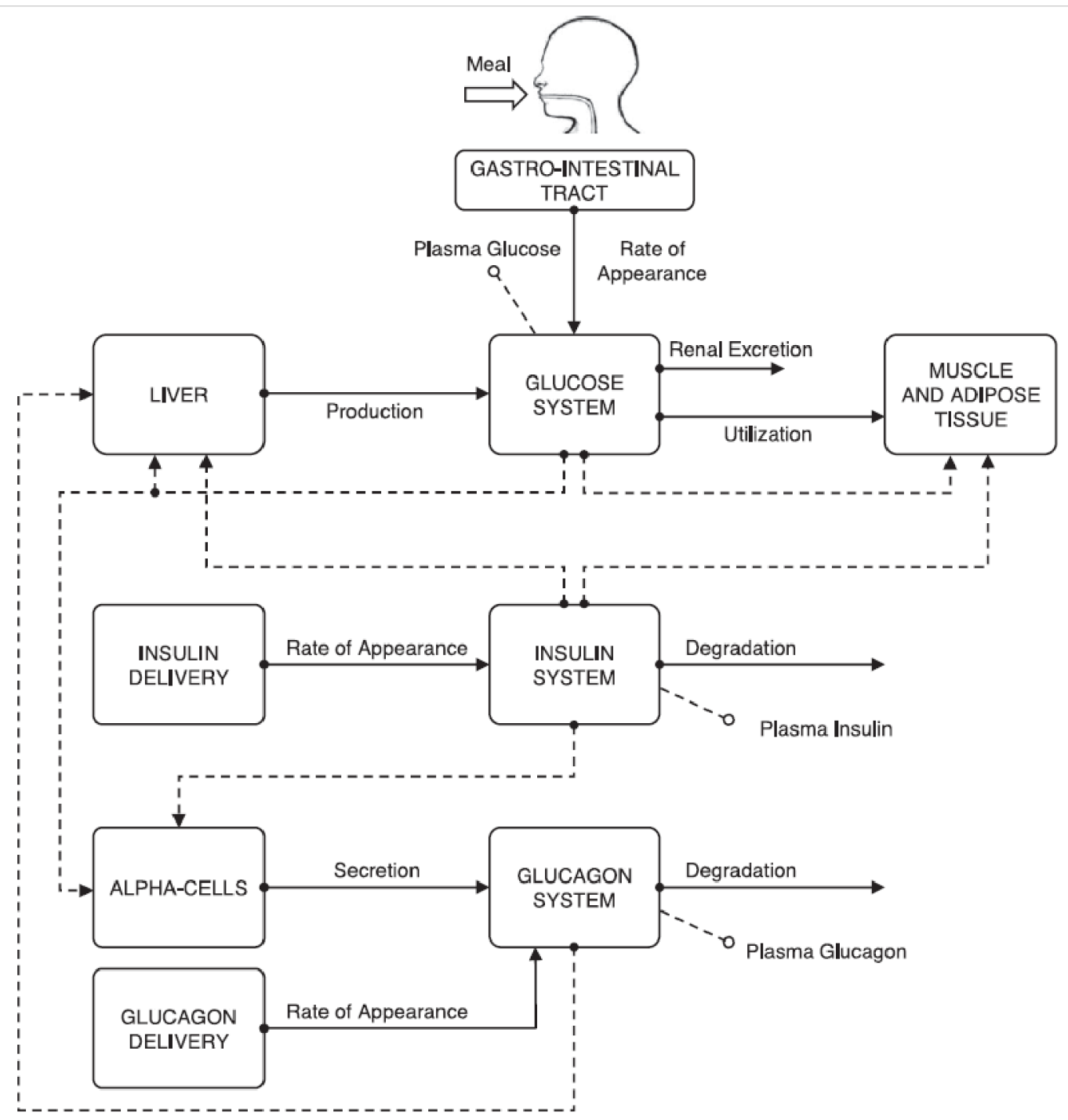

Figura 2.4: Diagrama del modelo de glucosa-insulina presentado por Dalla Man et al. Las líneas sólidas representan los flujos de glucosa e insulina, y las líneas punteadas las señales de control 75].

\section{Insulina}

Modelo de dos compartimentos (insulina en plasma y en hígado) que describe la cinética de la insulina.

\section{Variables}

- $I[\mathrm{pmol} / \mathrm{l}]:$ concentración de insulina en plasma

- $I_{p}[\mathrm{pmol} / \mathrm{kg}]:$ masa de insulina en plasma

- $I_{l}[\mathrm{pmol} / \mathrm{kg}]:$ masa de insulina en hígado

- $S[\mathrm{pmol} / \mathrm{kg} / \mathrm{min}]:$ secreción de insulina

\section{Parámetros}

- $m_{1}, m_{2}, m_{3}\left[\mathrm{~min}^{-1}\right]:$ parámetros de velocidad de la cinética de la insulina

- $m_{4}\left[\min ^{-1}\right]$ : parámetro debido a la degradación periférica, asumida como lineal 


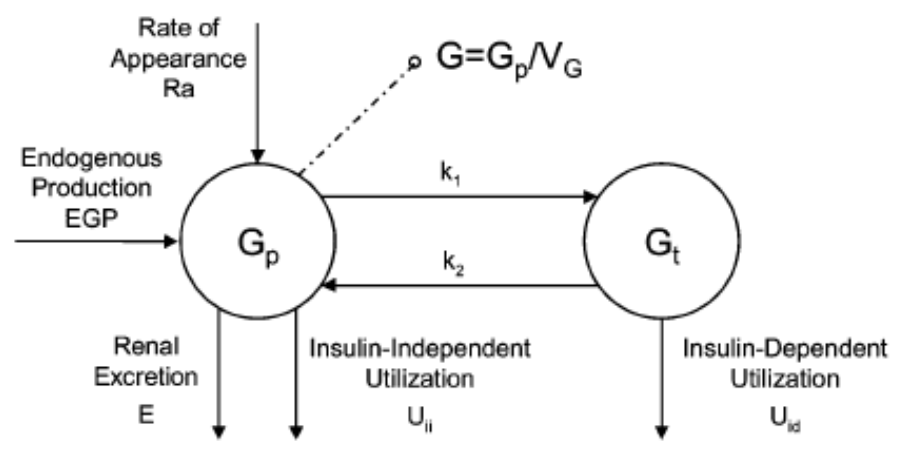

Figura 2.5: Subsistema que representa a la glucosa

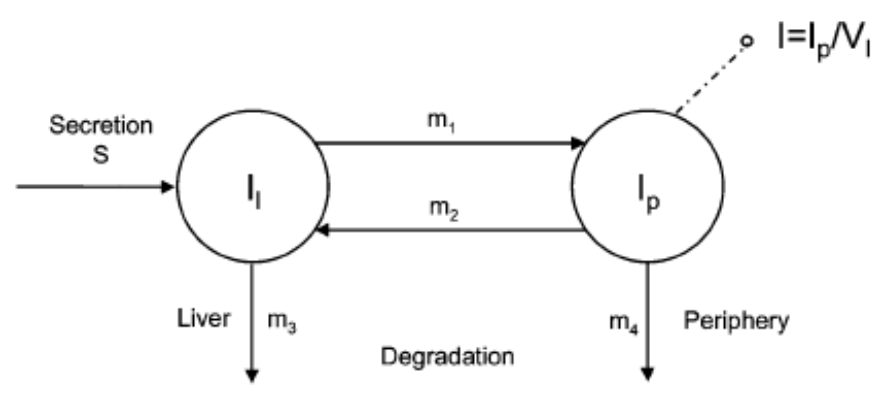

Figura 2.6: Subsistema que representa a la insulina

- $V_{I}[\mathrm{l} / \mathrm{kg}]$ : distribución del volumen de insulina

\section{Ecuaciones}

$$
\begin{cases}\dot{I}_{l}(t)=-\left(m_{1}+m_{3}(t)\right) \cdot I_{l}(t)+m_{2} \cdot I_{p}(t)+R a_{I}(t)+S(t) & I_{l}(0)=I_{l b} \\ \dot{I}_{p}(t)=-\left(m_{2}+m_{4}\right) \cdot I_{p}(t)+m_{1} \cdot I_{l}(t) & I_{p}(0)=I_{p b} \\ I(t)=\frac{I_{p}}{V_{I}} & I(0)=I_{b}\end{cases}
$$

La extracción hepática $H E(t)$ se vincula con la secreción de insulina, y hay que tener en cuenta que el hígado es responsable de la absorción del $60 \%$ de la insulina

$$
\begin{gathered}
H E(t)=-m_{5} \cdot S(t)+m_{6} \quad H E(0)=H E_{b} \\
m_{3}(t)=\frac{H E(t) \cdot m_{1}}{1-H E(t)} \quad H E(0)=H E_{b}
\end{gathered}
$$

\section{Producción endógena de glucosa}

Glucosa que produce el hígado en función de la glucosa y la insulina en plasma

\section{Variables}

- $I_{p o}[\mathrm{pmol} / \mathrm{kg}]$ : cantidad de insulina en la vena Porta (hígado)

- $I_{d}[\mathrm{pmol} / \mathrm{l}]:$ señal de la insulina retardada realizada con una cadena de dos compartimentos 


\section{Parámetros}

- $k_{p 1}[\mathrm{mg} / \mathrm{Kg} / \mathrm{min}]:$ EGP extrapolada a glucosa e insulina cero

- $k_{p 2}\left[\mathrm{~min}^{-1}\right]$ : efectividad de la glucosa del hígado

- $k_{p 3}[\mathrm{mg} / \mathrm{Kg} / \mathrm{min}$ per pmol/liter]: parámetro que gobierna la amplitud de la acción de la insulina en el hígado

- $k_{p 4}[\mathrm{mg} / \mathrm{kg} / \mathrm{min} /(\mathrm{pmol} / \mathrm{kg})]:$ parámetro que gobierna la amplitud de la acción de la insulina en la vena porta en el hígado

- $k_{i}\left[\min ^{-1}\right]$ : parámetro de tasa que cuantifica retraso entre la señal de la insulina y la acción de la insulina

\section{Ecuaciones}

$$
\begin{gathered}
E G P(t)=k_{p 1}-k_{p 2} \cdot G_{p}(t)-k_{p 3} \cdot X^{L}(t)+\zeta \cdot X^{H}(t) \\
E G P(0)=E G P_{b} \\
\begin{cases}\dot{X}^{L}(t)=-k_{i} \cdot\left[X^{L}(t)-I^{\prime}(t)\right] & X^{L}(0)=I_{b} \\
\dot{I}^{\prime}(t)=-k_{i} \cdot\left[I^{\prime}(t)-I(t)\right] & I^{\prime}(0)=I_{b}\end{cases} \\
\dot{X}^{H}(t)=-k_{H} \cdot X^{H}(t)+k_{H} \cdot \max \left[\left(H(t)-H_{b}\right), 0\right] \quad X^{H}(0)=0
\end{gathered}
$$

\section{Tasa de aparición de la glucosa}

Describe la transición de la glucosa en el estómago y el intestino, donde el estómago está representado por dos compartimentos (fase sólida $Q_{s t o 1}$ y triturada $Q_{s t o 2}$ ), mientras que un solo compartimiento describe al intestino $\left(Q_{\text {gut }}\right)$.

\section{Variables}

- $Q_{s t o}[\mathrm{mg}]$ : cantidad de glucosa en el estómago (fase sólida, $Q_{s t o 1}$ y líquida $Q_{s t o 2}$ )

- $Q_{g u t}[\mathrm{mg}]$ : masa de glucosa en el intestino

- $R a[\mathrm{mg} / \mathrm{kg} / \mathrm{min}]:$ tasa de aparición de glucosa en plasma

\section{Parámetros}

- $k_{\text {gri }}\left[\mathrm{min}^{-1}\right]$ : coeficiente de grado de "molido" que relaciona los primeros compartimientos

- $k_{\text {empt }}\left(Q_{s t o}\right)\left[\mathrm{min}^{-1}\right]$ : tasa de vacío gástrico hacia el intestino (función no lineal de $\left.Q_{\text {sto }}\right)$

- $k_{a b s}\left[\mathrm{~min}^{-1}\right]:$ tasa de absorción intestinal

- $f$ : es la fracción de absorción intestinal que aparece en plasma

- $B W[\mathrm{~kg}]:$ peso del cuerpo (body weight)

- $D[\mathrm{mg}]$ : cantidad de glucosa ingerida

- Resto de los parámetros arreglados según literatura o estimaciones 


\section{Ecuaciones}

$$
\begin{cases}Q_{s t o}(t)=Q_{s t o 1}(t)+Q_{s t o 2}(t) & Q_{s t o}(0)=0 \\ \dot{Q}_{s t o 1}(t)=-k_{g r i} \cdot Q_{s t o 1}(t)+D \cdot d(t) & Q_{s t o 1}(0)=0 \\ \dot{Q}_{s t o 2}(t)=-k_{\text {empt }}\left(Q_{s t o}\right) \cdot Q_{s t o 2}(t)+k_{g r i} \cdot Q_{s t o 1}(t) & Q_{s t o 2}(0)=0 \\ \dot{Q}_{g u t}(t)=-k_{a b s} \cdot Q_{g u t}(t)+k_{\text {empt }}\left(Q_{s t o}\right) \cdot Q_{s t o 2}(t) & Q_{g u t}(0)=0 \\ R a(t)=\frac{f \cdot k_{a b s} \cdot Q_{g u t}(t)}{B W} & R a(0)=0\end{cases}
$$

La tasa de vacío gástrico varía con el tiempo y depende de la cantidad total de glucosa en el estómago. En un comienzo es máximo $\left(k_{\max }\right)$ y luego decrece con una tasa $\alpha$ a su mínimo $k_{\min }$ para luego recuperar su máximo con tasa $\beta$.

$$
\begin{gathered}
k_{\text {empt }}\left(Q_{\text {sto }}\right)=k_{\text {min }}+\frac{k_{\text {max }}-k_{\text {min }}}{2}\left\{\tanh \left(\alpha\left(Q_{\text {sto }}(t)-b \cdot D\right)\right)-\tanh \left(\beta\left(Q_{\text {sto }}(t)-d \cdot D\right)\right)+2\right\} \\
\alpha=\frac{5}{2 \cdot D \cdot(1-b)} \quad \beta=\frac{5}{2 \cdot D \cdot c}
\end{gathered}
$$

\section{Utilización de la glucosa}

A partir de la literatura se construyó la utilización de la glucosa por los tejidos del cuerpo. Se asumieron dos componentes, un compartimiento insulina-independiente, el cual es constante $\left(F_{c n s}\right)$ y representa el uso del cerebro y de los eritrocitos (glóbulos rojos), y otro insulina-dependiente, y depende de forma no lineal de la glucosa en los tejidos.

\section{Variables}

- $U_{i i}[\mathrm{mg} / \mathrm{kg} / \mathrm{min}]:$ utilización de glucosa independiente de la insulina

- $U_{i d}[\mathrm{mg} / \mathrm{kg} / \mathrm{min}]:$ utilización de glucosa dependiente de la insulina

- $X(t)[\mathrm{pmol} / \mathrm{L}]$ : insulina en el fluido intersticial

- $F_{c n s}[\mathrm{mg} / \mathrm{kg} / \mathrm{min}]:$ glucosa consumida por el cerebro y los glóbulos rojos

\section{Parámetros}

- $V_{m 0}[\mathrm{mg} / \mathrm{kg} / \mathrm{min}]:$ parámetro de la ecuación Michaelis-Menten de utilización de glucosa ante acción nula de la insulina

- $K_{m 0}[\mathrm{mg} / \mathrm{kg}]:$ parámetro de la ecuación Michaelis-Menten de utilización de glucosa ante acción nula de la insulina

- $V_{m x}[\mathrm{mg} / \mathrm{kg} / \mathrm{min}$ per pmol/liter]: sensibilidad insulínica

- $p_{2 U}\left[\mathrm{~min}^{-1}\right]$ : tasa constante de la acción en la utilización de glucosa periférica

\section{Ecuaciones}

$$
\begin{gathered}
U(t)=U_{i i}(t)+U_{i d}(t) \\
U_{i i}(t)=F_{c n s}
\end{gathered}
$$




$$
\begin{gathered}
U_{i d}=\frac{\left[V_{m 0}+V_{m x} \cdot X(t) \cdot\left(1+r_{1} \cdot r i s k\right)\right] \cdot G_{t}(t)}{K_{m 0}+G_{t}(t)} \\
\dot{X}(t)=-p_{2 U} \cdot X(t)+p_{2 U}\left[I(t)-I_{b}\right] \quad X(0)=0 \\
r i s k=\left\{\begin{array}{lll}
0 & \text { si } G \geq G_{b} \\
10 \cdot[f(G)]^{2} & \text { si } & G_{t h} \leq G<G_{b} \\
10 \cdot\left[f\left(G_{t h}\right)\right]^{2} & \text { si } & G<G_{t h}
\end{array}\right.
\end{gathered}
$$

\section{Excreción renal de glucosa}

La extracción renal de glucosa por el riñón ocurre cuando la glucosa en plasma excede cierto umbral

\section{Variable}

- $E(t)[\mathrm{mg} / \mathrm{kg} / \mathrm{min}]:$ Excreción de glucosa por los riñones

\section{Parámetros}

- $k_{e 1}\left[\mathrm{~min}^{-1}\right]$ : tasa de filtración glomerular

- $k_{e 2}[\mathrm{mg} / \mathrm{kg}]$ : umbral de glucosa renal

\section{Ecuaciones}

$$
E(t)= \begin{cases}k_{e 1} \cdot\left[G_{p}(t)-k_{e 2}\right] & \text { si } G_{p}(t)>K_{e 2} \\ 0 & \text { si } G_{p}(t) \leq K_{e 2}\end{cases}
$$

\section{Cinética de la insulina subcutánea}

Para simular un paciente diabético tipo I se sustituye el módulo de secreción por uno de infusión subcutánea de insulina.

\section{Variables}

- $I_{s c 1}[\mathrm{pmol} / \mathrm{kg}]$ : cantidad de insulina no-monomérica en el espacio subcutáneo

- $I_{s c 2}[\mathrm{pmol} / \mathrm{kg}]$ : cantidad de insulina monomérica en el espacio subcutáneo

- $\operatorname{IIR}(t)[\mathrm{pmol} / \mathrm{kg} / \mathrm{min}]:$ tasa de infusión exógena de insulina

\section{Parámetros}

- $k_{d}\left[\min ^{-1}\right]:$ tasa de disociación de insulina

- $k_{a 1}\left[\mathrm{~min}^{-1}\right]$ : tasa de absorción de insulina no-monomérica

- $k_{a 2}\left[\min ^{-1}\right]:$ tasa de absorción de insulina monomérica 


\section{Ecuaciones}

$$
\begin{gathered}
\left\{\begin{array}{cc}
\dot{I}_{s c 1}(t)=-\left(k_{d}+k_{a 1}\right) \cdot I_{s c 1}(t)+I I R(t) & I_{s c 1}(0)=I_{s c 1 s s} \\
\dot{I}_{s c 2}(t)=k_{d} \cdot I_{s c 1}(t)-k_{a 2} \cdot I_{s c 2}(t) & I_{s c 2}(0)=I_{s c 2 s s}
\end{array}\right. \\
R_{i}(t)=k_{a 1} \cdot I_{s c 1}(t)+k_{a 2} \cdot I_{s c 2}(t)
\end{gathered}
$$

\section{Glucosa subcutánea}

$$
\dot{G}_{s c}(t)=-1 / T_{s} \cdot G_{s c}+1 / T_{s} \cdot G(t) \quad G_{s c}(0)=G_{b}
$$

\section{Cinética del Glucagón y su secreción}

$$
\begin{gathered}
\dot{H}(t)=-n \cdot H(t)+S R_{H}(t)+R a_{H}(t) \quad H(0)=H_{b} \\
S R_{H}(t)=S R_{H}^{s}(t)+S R_{H}^{d}(t) \\
\dot{S} R_{H}^{s}(t)= \begin{cases}-\rho \cdot\left[S R_{H}^{s}(t)-S R_{H}^{b}\right] & \text { si } G(t) \geq G_{b} \\
-\rho \cdot\left[S R_{H}^{s}(t)-\max \left(\frac{\sigma \cdot\left[G_{t h}-G(t)\right]}{I(t)+1}+S R_{H}^{b}, 0\right)\right] & \text { si } G(t)<G_{b}\end{cases} \\
S R_{H}^{d}(t)=\delta \cdot(\max )\left(-\frac{d G(t)}{d t}, 0\right)
\end{gathered}
$$

\section{Cinética de Glucagón subcutáneo}

$$
\begin{gathered}
\begin{cases}\dot{H}_{s c 1}(t)=-\left(k_{h 1}+k_{h 2}\right) \cdot H_{s c 1}(t) & H_{s c 1}(0)=H_{s c 1 b} \\
\dot{H}_{s c 2}(t)=k_{h 1} \cdot H_{s c 1}(t)-k_{h 3} \cdot H_{s c 2}(t) & H_{s c 2}(0)=H_{s c 2 b}\end{cases} \\
R a_{H}(t)=k_{h 3} \cdot H_{s c 2}(t)
\end{gathered}
$$

\subsubsection{Comparación de modelos}

La comparación de los modelos se presenta en términos de su comportamiento a lazo abierto, comparando ventajas y desventajas a la hora de su implementación. En la literatura hay diversas revisiones de los modelos desarrollados 80,81, y discusiones de los ensayos in-silico 82, 83. En el cuadro 2.1 se resumen los aspectos de cada modelo estudiado.

El modelo de Sorensen fue el primero que presentó una dinámica compartimental completa que con la idea de un paciente promedio, podría ser representado paramétricamente. Éste permite una inmediata trasformación de una persona normal a un sujeto con DMT1 eliminando el factor asociado a la secreción de insulina del páncreas $\Gamma_{I R}$ y contempla el efecto del glucagón, a diferencia del modelo de Bergman y el de Cambridge. Sin embargo, posee varias desventajas. Sólo contempla insulina intravenosa, perdiéndose el gran retardo de la inyección de insulina de forma subcutánea, lo que resulta muy importante a tener en cuenta a la hora de realizar un control de lazo cerrado. Aún así, el submodelo compartimental de absorción de insulina subcutánea podría añadirse sin perjudicar las dinámicas del modelo. Aunque la capacidad del modelo para predecir anormalidades metabólicas ha sido demostrada, se reconoce que un ajuste de parámetros es necesario, lo cual derivó, en estudios posteriores, a compensar la falta de variación inter-paciente variando ciertos 
parámetros fisiológicos. Sin embargo, éstos fueron sintetizados mediante el modelo a falta de datos clínicos [81].

El modelo de Cambridge está diseñado para contemplar el desarrollo de controladores de lazo cerrado para pacientes con DMTI. Las predicciones poblacionales del simulador han sido validadas por estudios clínicos en sujetos jóvenes con DMTI en pruebas de noche a la mañana [70]. Como problemática, posee que el modelo de absorción gostrointestinal de glucosa es simplista, en comparación al del grupo UVA/Padova.

El modelo utilizado en el simulador UVA/Padova está también basado en compartimentos. En contraste con el modelo de Sorensen, resuelve el problema de la variabilidad inter-paciente mediante una gran cohorte de sujetos virtuales. Posee un detallado modelo de absorción de glucosa y modelos que representan el monitoreo de glucosa y la administración subcutánea de insulina, lo que implica simulaciones más realistas. Como principal atractivo para la comunidad científica es que ha sido aceptado por la FDA como sustituto de pruebas pre-clínicas en animales para controladores de lazo cerrado.

El modelo de Bergman posee como fortaleza parámetros variantes con el tiempo, pero está limitado por la representación simplista de la cinética de la glucosa y una sobretimación de su efectividad. Se han desarrollado modelos más completos a partir del mismo agregando subsistemas desarrollados por otras entidades, como en el caso de Fabietti y Medtronic [84, los cuales se han obviado en este análisis.

Según [1], se considera que los principales componentes de un modelo de simulación para la validación efectiva de un controlador de glucosa se resumen en:

1. Un modelo de la cinética de la glucosa y de la acción de la insulina, con complejidad suficiente para representar adecuadamente dicha dinámica y simplicidad suficiente para que sus parámetros puedan ser identificados a partir de ensayos clínicos.

2. Un modelo de la cinética de la insulina en el espacio subcutáneo. Actualmente las bombas de insulina suministran la insulina de forma discreta en el espacio s.c., por lo que es necesario considerar el retraso temporal al trasporte hacia el plasma.

3. Un modelo de la absorción de glucosa en el intestino capaz de representar distintos patrones de absorción de comidas de composición mixta (no únicamente carbohidratos).

4. Un modelos específico de los errores asociados a los sensores continuos de glucosa, que sea capaz de reproducir los retrasos temporales debidos a la lectura en el espacio s.c., las desviaciones propias del sistema y a la calibración, y el ruido aleatorio de los sensores.

5. Un modelo del efecto del ejercicio en la cinética de la glucosa. Aunque la mayor parte de los entornos de simulación actualmente disponibles no incluyen este componente, su inclusión representaría una aportación significativa.

6. Una población de pacientes virtuales lo suficientemente representativa de la variabilidad intra e inter-paciente de los parámetros metabólicos en la población general de sujetos con DMT1. Ésta es una característica que desafortunadamente no incluyen todos los simuladores existentes y que en otros casos se debería mejorar significativamente.

Como conclusión tras la comparación de los diferentes modelos matemáticos, se optó por implementar el modelo UVA/Padova, debido a que cumple de manera adecuada muchas de las condiciones anteriormente mencionadas, teniendo varias ventajas respecto al resto (resaltadas en la tabla 2.1). El mismo se encuentra en continuo desarrollo y tiene la posibilidad de implementarse variación intra-paciente. 
Tabla 2.1: Comparación de los modelos del sistema glucosa-insulina presentados en la literatura.

\begin{tabular}{|c|c|c|}
\hline Modelo & Ventajas & Desventajas \\
\hline \multicolumn{3}{|l|}{ Sorensen } \\
\hline & $\begin{array}{l}\text { - Transformación inmediata de } \\
\text { un paciente normal a uno con } \\
\text { DMTI } \\
\text { - Considera la dinámica del } \\
\text { glucagón }\end{array}$ & $\begin{array}{l}\text { - La administración de insuli- } \\
\text { na es intravenosa } \\
\text { - La variabilidad inter e intra- } \\
\text { paciente no es considerada }\end{array}$ \\
\hline \multicolumn{3}{|l|}{ Bergman } \\
\hline & $\begin{array}{l}\text { - Posee parámetros que varían } \\
\text { con el tiempo }\end{array}$ & $\begin{array}{l}\text { - Representación muy simplis- } \\
\text { ta de la cinética de la glucosa } \\
\text { - Pequeña duración de la ac- } \\
\text { ción de la insulina } \\
\text { - Sobre estimación de la efecti- } \\
\text { vidad de la glucosa }\end{array}$ \\
\hline \multicolumn{3}{|l|}{ Hovorka } \\
\hline & $\begin{array}{l}\text { - Posee pacientes validados } \\
\text { con estudios clínicos } \\
\text { - Variabilidad intra-paciente } \\
\text { - Agrega modelos generales de } \\
\text { monitoreo de glucosa y de } \\
\text { bombas insulina }\end{array}$ & $\begin{array}{l}\text { - La dinámica del glucagón no } \\
\text { es considerada } \\
\text { - La absorción gastrointestinal } \\
\text { de la glucosa es muy simple }\end{array}$ \\
\hline $\begin{array}{l}\text { Dalla } \\
\text { Man }\end{array}$ & $\begin{array}{l}\text { - Variación inter-paciente } \\
\text { - Posee gran cantidad de suje- } \\
\text { tos virtuales } \\
\text { - El modelo de absorción de } \\
\text { glucosa es realista } \\
\text { - Agrega modelos de monito- } \\
\text { reo de glucosa y de bombas } \\
\text { de insulina } \\
\text { - Incluye a la dinámica del glu- } \\
\text { cagón } \\
\text { - Se han desarrollado varai- } \\
\text { ciones paramétricas para } \\
\text { representar variación intra- } \\
\text { paciente } \\
\text { - Aceptado por la FDA }\end{array}$ & $\begin{array}{l}\text { - No se considera la variabili- } \\
\text { dad intra-paciente }\end{array}$ \\
\hline
\end{tabular}

\subsection{Plataforma de simulación UNLP}

El diseño de una plataforma propia de simulación parte de las limitaciones que ofrecía la versión de distribución del simulador UVA/Padova a la cual se podía acceder. Entre estas, se encontraban la limitada accesibilidad a la estructura del simulador, la dificultad de cambiar ciertos parámetros de simulación (por ejemplo el tiempo de muestreo) y la 
versatilidad de la plataforma a la inclusión de nuevos módulos o bloques que hicieran más realistas las pruebas in-silico.

Se partió de la estructura del simulador UVA/Padova versión S2008 [73] y de la versión S2013 75] realizada en el entorno MATLAB ${ }^{\circledR}$. A partir de un análisis de los componentes y funciones que integran a la plataforma, se obtuvo un panorama del marco de simulación, conformado por los siguientes elementos :

- Escenario de simulación (incluyendo terapia, comidas, eventos, entre otros).

- Configuración de la simulación del entorno Simulink.

- Creación y administración vectores de bolos de insulina y comidas.

- Implementación del modelo de DMT1 y carga de los parámetros del modelo.

- Modelos correspondientes a los dispositivos bomba de insulina y sensor de glucosa.

- Bloque de implementación de controlador.

- Representación de los resultados de forma gráfica.

Los siguientes criterios se tuvieron en cuenta a la hora de desarrollar una nueva plataforma:

- Estructura didáctica en base a bloques específicos.

- Programación de funciones determinadas para cada bloque, con objetivos específicos.

- Desarrollo intuitivo para el usuario de las acciones de cada bloque y el proceder de cada función, para su manipulación/modificación.

- Fácil acceso de los parámetros tanto de simulación como del sistema.

- Capacidad de simular a diferentes valores de paso fijo.

- Una interfaz gráfica intuitiva y ágil que no limite la versatilidad del simulador. A su vez que pueda omitirse y realizar simulaciones mediante una estructura de parámetros.

- Variedad de muestras gráficas de los resultados, con posibilidad de generar nuevos gráficos sin necesidad de volver a correr la simulación y sencillez de agregar gráficos por el usuario.

- Introducir diferentes fuentes de variabilidad intra-paciente

- Introducir el efecto de comidas mixtas

\subsubsection{Bloques}

Los bloques de la estructura del programa que dan el aspecto didáctico, intuitivo y estructurado que se consideraron apropiados fueron:

Bloque de inicialización: se crean las estructuras a utilizar y se les asigna espacio de memoria para mayor eficiencia.

Bloque de configuración de simulación: se utiliza una interfaz gráfica (Fig. 2.7) donde el usuario podrá determinar:

- Sujetos a simular. 
- Elección de escenario, diseñado previamente a partir de un archivo ASCII.

- Paso de simulación.

- Tiempo de simulación.

- Elección de hardware (bomba de insulina y sensor de glucosa).

- Optar por añadir comidas mixtas.

- Optar por incluir intra-paciente. En caso de incluir se abre una nueva ventana de sección de clase de variación.

- Determinar los rangos de hipoglucemia e hiperglucemia.

- Seleccionar tipo de tratamiento (lazo abierto, cerrado o híbrido).

Esta a su vez puede omitirse y estos parámetros pueden configurarse mediante una estructura dentro del script.

Bloque de creación del entorno: a partir de las condiciones definidas en el bloque anterior y en el escenario, se crean los vectores de las comidas, bolos de insulina manuales y óptimos, insulina basal, insulina y glucosa intravenosa, variación intra-paciente, comidas mixtas, que serán utilizados a lo largo de la simulación. Los vectores creados poseen el valor de la variable para cada paso de simulación.

Bloque del controlador: se previó un subsistema para el diseño de un futuro controlador, y la posible adaptación de un tratamiento de lazo cerrado. Al mismo se le adjuntaron como entradas la glucosa subcutánea y el anuncio de comidas, siendo las salidas la insulina basal y los bolos propiamente dichos. Este subsistema es totalmente modificable por el usuario. Para la sintonización del controlador, sólo ciertos parámetros clínicos de los sujetos son accesibles, siendo los parámetros del modelo inaccesibles.

Bloque de simulación: dentro de este bloque, se crea un bucle de acuerdo a la cantidad de sujetos seleccionados. Se cargan los datos del primer sujeto y se lo simula en la plataforma de Simulink. Luego se guardan los resultados y variables obtenidos y se repite para el siguiente sujeto, hasta completar todos los pacientes seleccionados anteriormente.

Bloque de resultados: Terminadas las simulaciones, resulta muy importante guardar los datos obtenidos de forma práctica y accesible, tanto para un análisis inmediato como para un futuro análisis más profundo. Para el análisis inmediato se ofrece una serie de diferentes gráficos y una tabla con valores estadísticos. A su vez, todos los vectores creados, los estados y salidas del sistema, los parámetros de configuración de la simulación.

\section{Escenarios}

Para la creación del entorno de simulación, se partió de los escenarios que utiliza el simulador UVA/Padova, además que esto agrega la fácil comparación y verificación entre los resultados de las simulaciones de ambos simuladores. Estos consisten en la determinación por el usuario de las condiciones a las que se someterá al/a los sujeto/s a simular. En los mismos se define:

- Horario de cada comida.

- Cantidad de CHO de cada comida.

- Duración de las comidas.

- Tiempo en que se suministrarán las dosis de insulina. 

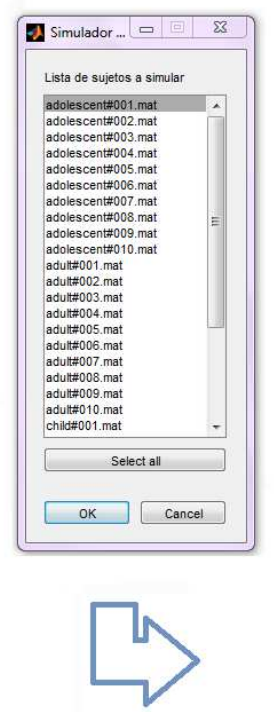
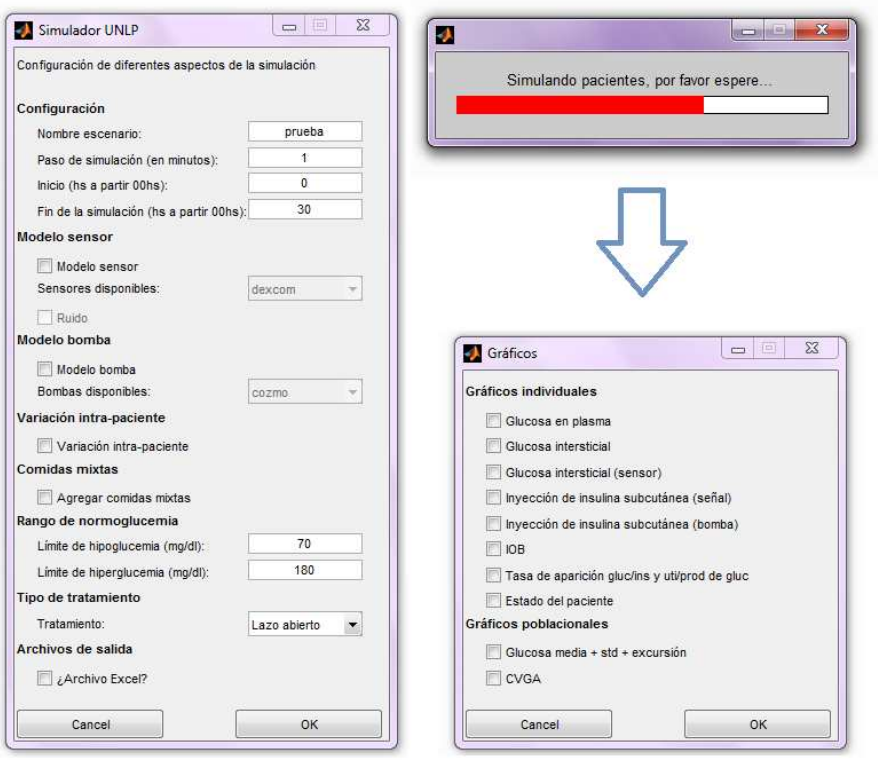

Figura 2.7: Interfaz principal de la plataforma de simulación UNLP.

- Cantidad de insulina de cada bolo a suministrar.

- Tiempo y cantidad de suministros intravenosos al paciente de glucosa y/o insulina.

- Capacidad de suministro de bolos óptimos en función de la sensibilidad insulínica de cada paciente y el anuncio de comidas.

- Glucosa inicial y glucosa objetivo.

\section{Simulink}

A la hora de implementar el modelo de estados del sujeto dentro de Simulink se optó por el bloque S-Function que permite describir modelos continuos no lineales variantes en el tiempo.

El bloque S-Function consiste en una serie de entradas, estados, y salidas, donde las últimas son en función del tiempo de simulación, los estados del sistema y las entradas. Es utilizado para simular sistemas continuos, discretos o híbridos. Un modelo implementado mediante S-Function de Simulink procede en pasos. En primer lugar viene la fase de inicialización, donde el simulador incorpora los bloques de bibliotecas al modelo, propaga los valores de las señales, los tipos de datos, tiempos de muestreo, evalúa los bloques de parámetros, determina el orden de ejecución de los bloques y asigna la memoria. Luego, el simulador entra en un bucle de simulación. Durante cada paso, se ejecuta cada bloque del modelo en el orden determinado en la inicialización. Para cada bloque, se llama a las funciones que calculan los bloques de estados, derivadas y salidas correspondientes a la muestra de tiempo actual. Los estados definidos dentro del bloque corresponden a los del modelo presentado en 75$]$.

Se definieron las siguientes entradas al modelo:

- CHO sólido consumido [mg]

- Insulina subcutánea $[\mathrm{pmol} / \mathrm{Kg}]$

- Insulina intravenosa $[\mathrm{pmol} / \mathrm{Kg}]$

- Glucosa intravenosa (dextrosa) $[\mathrm{mg} / \mathrm{Kg}]$ 
- Tasa de aparición de comidas mixtas $[\mathrm{mg} / \mathrm{kg} / \mathrm{min}]$

- Glucagón intravenoso

- Glucagon subcutáneo

y las siguientes salidas:

- Glucosa intersticial

- Insulina en plasma

- Producción de glucosa

- Tasa de aparición de la glucosa

- Utilización de la glucosa

- Tasa de aparición de la insulina

- Glucosa en plasma

- Los estados del sistema

Al paciente lo acompañan el modelo de la bomba de insulina, el modelo del sensor continuo de glucosa y el controlador de lazo cerrado.

El modelo de la bomba incluye sus limitaciones técnicas principales: discretización, cuantización, error, valores máximos y mínimos tanto de bolo como de suministro basal.

El modelo de sensor continuo de glucosa concierne a: valor mínimo medible, valor máximo, tasa de muestreo, un reconstructor de orden cero, offset, ganancia, retardo y ruido. El ruido del sensor $\epsilon_{n}$ no es blanco ni Gaussiano, sino que es modelado por un proceso de promedio móvil autoregresivo el cual fue estimado usando datos clínicos en [85]. El error del sensor es calculado usando la transformación de Johnson a partir de los parámetros del sensor $\xi, \lambda, \gamma$ y $\delta[85]$ y del modelo dado por:

$$
\begin{aligned}
& e_{0}=v_{0} \\
& e_{n}=0,7 *\left(e_{n-1}+v_{n}\right) \\
& \epsilon_{n}=\xi+\lambda \sinh \left(\frac{e_{n}-\gamma}{\delta}\right)
\end{aligned}
$$

donde $v_{n} \phi(0,1)$ es independiente e idénticamente distribuido.

\section{Resultados de la simulación}

Los resultados obtenidos deben ser presentados de forma apropiada al usuario, facilitando el análisis del ensayo realizado y la obtención de conclusiones. Finalizada la simulación, la información colectada se guarda en un archivo propio del Matlab de formato .mat conteniendo todos los parámetros y vectores utilizados en la simulación para cada sujeto. Esto permite verificar después de realizada la simulación aspectos como el escenario utilizado, los sujetos simulados, los parámetros y vectores creados y/o modificados por el programa. En un archivo tabla formato .xls se guardan datos utilizados para el análisis cuantitativo de la simulación, guardándose para cada paciente el valor medio, máximo y mínimo de la glucosa en plasma, el tiempo en hiperglucemia e hipoglucemia, y el número de hipoglucemias. Respecto a los resultados gráficos, se podrán seleccionar gráficos individuales de todas las salidas definidas, pero también gráficas poblacionales como la glucosa promedio y desviación estándar, valores máximos y mínimos, y un gráfico Control Variability Grid Analysis (CVGA) utilizado típicamente en estos ensayos (coloca el rendimiento del algoritmo de control en algún área en particular según un periodo de observación). Cada punto representa los valores mínimos/máximos obtenidos en la excursión de glucosa [86]. 


\subsubsection{Variación intra-paciente}

Los procesos biológicos se caracterizan por tener una amplia variabilidad, que puede presentarse con ritmos de tipo ultradiana (periodos menores a las 24 horas), circadiana (periodos de 24 horas) o circanuales (periodos de 365 días) 87]. En el caso de la DMT1 los requerimientos de insulina para controlar los niveles de GP varían durante el día. Esto se debe a cambios circadianos en dos importantes aspectos del sistema glucosa-insulina 88]:

- Tolerancia a la Glucosa (TG): hace referencia a la cantidad de glucosa que toman los tejidos periféricos.

- Sensibilidad Insulínica (SI): corresponde a la habilidad de la insulina a estimular la utilización de glucosa por parte de los tejidos e inhabilitar su producción por parte del hígado.

Por lo general, se considera que durante la mañana, los requerimientos de insulina son mayores en las personas con diabetes, en comparación con el resto de las comidas. Este cambio se lo asocia al denominado "Efecto Amanecer" (Dawn Phenomenon), el cual describe un estado de hiperglucemia durante horas de la madrugada [89]. Este fenómeno representa una gran variabilidad entre los sujetos y puede verse afectado por diversos factores, como la duración de la diabetes o calidad del control glucémico. Se lo puede relacionar tanto a cambios en la SI como de la TG. Sin embargo, la TG también puede relacionarse y regularse mediante la SI, por lo tanto, la variación intra-paciente puede describirse mediante una variación circadiana apropiada de la SI [90].

La variación intra-paciente se modela mediante una descripción de variación circadiana de la SI que contemple hora del día, la actividad física, estrés y otros factores que se crean convenientes. Este enfoque es el que se tiene en cuenta a la hora de representar variaciones en los modelos de DMT1 88. Varios propuestas fueron realizadas para incluir la variación intra-paciente en la literatura 69,77,91, 94].

En la plataforma desarrollada se tuvieron en cuenta 3 maneras diferentes de implementar la variación en la SI en el modelo de UVA/Padova. Cabe destacar que más adelante durante el trascurso del doctorado también se tuvo en cuenta los efectos del ejercicio sobre la dinámica de la glucosa, a lo que el lector puede referirse en el Capítulo 3.

La SI en el modelo de UVA/Padova, presentado en la sección 2.1.4, se ve representada por los siguientes parámetros:

- $V_{m x}$ : relaciona con la utilización dependiente de la insulina (ec. 2.24)

- $k_{p 3}$ : gobierna la amplitud de la acción de la insulina sobre el hígado (ec. 2.17)

Los métodos descritos a continuación pueden seleccionarse y alternarse en la plataforma. Debido a la variación temporal de los parámetros asociados a la [SI, los perfiles de insulina basal deben variar a lo largo del día para mantener estable la glucosa en ayunas. Se considera que una variación menor a 30mg/dl alrededor del nivel objetivo (100-130 $\mathrm{mg} / \mathrm{dl}$ ) es deseable 14 . Por ejemplo, en la Figura 2.8 pueden verse los perfiles basales promedio por rango etario según 95 .

Cada uno de los métodos implementados en la plataforma UNLP genera perfiles de variación diferentes y a su vez modifican el cálculo de los perfiles de insulina basal de los sujeto y el coeficiente CR. Los perfiles basales son generados automáticamente para cada sujeto para contrarrestar la variación de la SI.

\section{Variación sinusoidal}

Un enfoque que considera variación paramétrica consiste en implementar variaciones sinusoidales. Esta forma fue implementada en 96 para representar variaciones en la SI y 


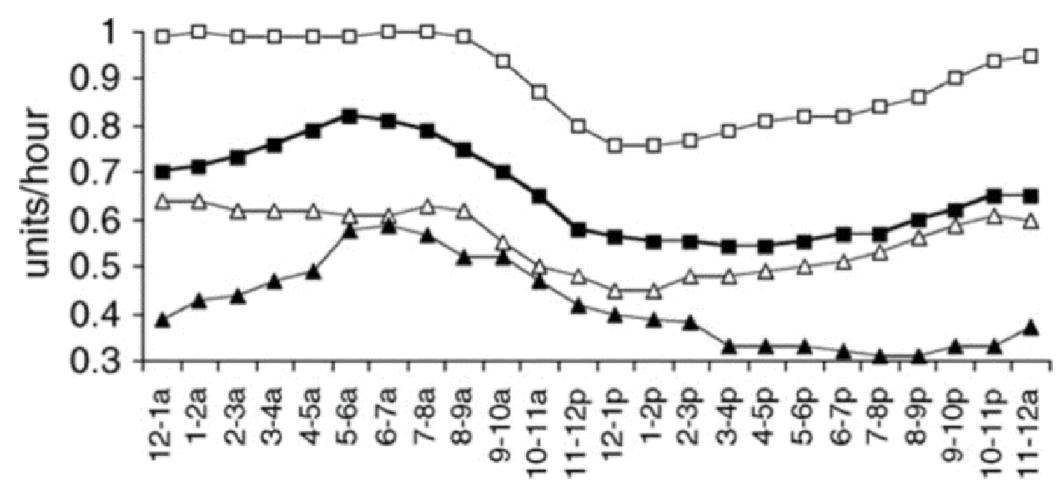

Figura 2.8: Perfiles basales promedio por rango etario. Triángulos claros: 3-10 años. Cuadrados claros: 11-20 años. Cuadrados oscuros: 20-60 años. Triángulos oscuros: $>60$ años 95 .

en la absorción de insulina en el espacio subcutáneo, y también fue considerada en [93], ambas propuestas sobre el modelo de UVA/Padova.

En esta plataforma se propone afectar los parámetros asociados a la SI del modelo UVA/Padova mediante el siguiente perfil variante en el tiempo:

$$
p(t)=p_{0}+p_{0} \cdot K(t) \sin \left(\frac{2 \pi}{P \cdot 60}+2 \pi F\right)
$$

donde $p(t)$ representa el parámetro que se desea variar en el tiempo, $p_{0}$ es el valor por defecto del parámetro, $P$ determina el periodo de la variación (24 para una variación circadiana), $F$ permite ajustar la fase pudiéndose ajustar manualmente o aleatoriamente mediante una distribución uniforme en el intervalo [0,1] y por último $K(t)$ permite ajustar la amplitud de la variación en términos de $p_{0}$, pudiéndose ajustar de manera fija o variante en el tiempo.

Además de considerar este mecanismo de variación paramétrica sobre la SI, también se consideró una variación sinusoidal en los parámetros $k_{d}, k_{a 1}, k_{a 2}$ de absorción de insulina subcutánea (ec. (2.28) ) para tener en cuenta las variaciones a corto plazo del DIA 97.

\section{Variación a partir de perfiles basales}

Se incluyó un perfil de variación circadiana en la SI para cada sujeto adulto basada en perfiles clínicos de insulina basal de pacientes adultos presentados en [95] (Fig. 2.8). Para este fin, el parámetro $V_{m x}$ fue modificado para que la excursión de glucosa en ayunas se corresponda con un perfil de insulina basal dado. En la Fig. 2.9 se ejemplifica la excursión de glucosa en ayunas de un sujeto virtual bajo variación circadiana de la SI. En la Fig. 2.10 puede observarse el promedio de los 10 perfiles de variación (correspondientes a los 10 sujetos adultos de la versión de distribución del UVA/Padova) que afectan al parámetro $V_{m x}$. Puede notarse una gran similitud al perfil de variación de SI presentado en 92. En [96 se puede apreciar la insulina basal por hora y los valores de los coeficientes CR utilizados para cada paciente adulto.

\section{Variación mediante patrones}

Para tener en cuenta una variación más realista y versátil, se consideró la variabilidad diurna de los parámetros del sistema que describe la sensibilidad a la insulina $\left(V_{m x}\right.$, $\left.k_{p 3}\right)$ siguiendo el trabajo de Visentin et al. 77, 78, 99. La variabilidad intradiaria de los parámetros variables en el tiempo, tanto de la SI como de la absorción de glucosa, se implementó como una señal casi escalonada que varía tres veces al día: a las 4, 11 y 17 horas (en referencia a tres momentos del día: Breakfast, Lunch y Dinner). Los cambios de 


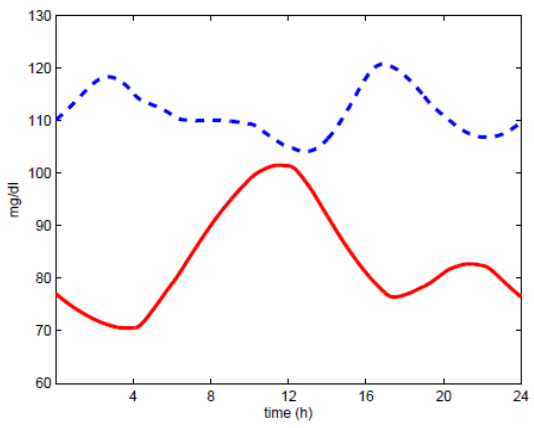

(a)

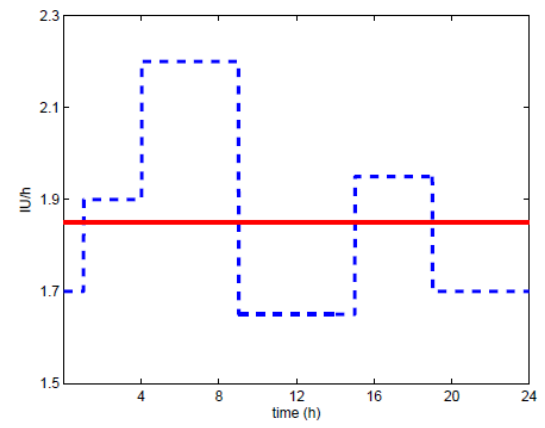

(b)

Figura 2.9: Concentración de glucosa de un sujeto virtual incluyendo variación en la SI (a) de acuerdo al perfil de insulina basal aplicado (b). La línea punteada azul corresponde a un perfil variable y la línea sólida roja a un perfil constante 98 .

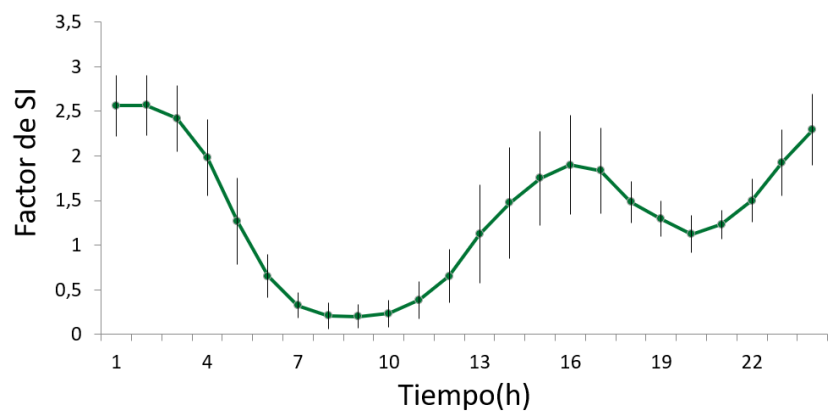

Figura 2.10: Media y desviación estándar del parámetro de variación aplicado sobre $V_{m x}$ sobre los 10 sujetos adultos.

sensibilidad se suavizaron por medio de una función sigmoide. En el cuadro 2.2 se muestran los patrones correspondientes a cada clase y la probabilidad de que un sujeto caiga en esa clase. En la Fig. 2.11 se ejemplifica el perfil correspondiente a la clases 5 que afecta a los parámetros de SI.

Cada sujeto virtual es asignado de forma aleatoria a un perfil de clase de SI variable en el tiempo, tal y como se presenta en 77 . Los parámetros involucrados con SI se variaron entre $100 \%$ y $60 \%$. A su vez se tiene en cuenta un ruido aleatorio multiplicativo, descrito por una distribución normal $N(\mu, \sigma)$, con valores nominales de $\mu=1$ y $\sigma=0,2$. Tanto la amplitud de la variación como los parámetros de la distribución del ruido pueden variarse a gusto.

Se definieron patrones diarios de la tasa de insulina basal variable en el tiempo y los coeficientes CR para compensar el patrón de SI del sujeto. La tasa basal de los pacientes se ajustó para mantener la excursión de glucosa estable en ayunas. Se establecieron dos niveles que afectan al perfil basal del sujeto: uno cuando los parámetros de la SI están en $100 \%$ y otro cuando están en $60 \%$. El ruido no fue considerado para el cálculo de la insulina basal. Esto permite simular a los sujetos en condiciones no ideales de tratamiento bajo variación intra-paciente. El cambio de la dosis basal se produce dos horas antes de que se definiera el cambio de sensibilidad, siendo modificable a gusto. Los diferentes CR para cada momento del día se definieron como $100 \%$ o $60 \%$ del valor nominal, de acuerdo al tipo de clase. 


\begin{tabular}{|c|c|c|c|}
\hline Class & IS pattern & IS profile & Probability \\
\hline 1 & $\begin{array}{c}l-l-l \text { or } \\
h-h-h\end{array}$ & $\longrightarrow$ & 0.1 \\
\hline 2 & $h-h-l$ & B & 0.05 \\
\hline 3 & $h-l-h$ & B & 0.05 \\
\hline 4 & $h-l-l$ & B & 0.1 \\
\hline 5 & $l-h-h$ & B & 0.2 \\
\hline 6 & $l-h-l$ & $\mathrm{~B}$ & 0.2 \\
\hline 7 & $l-l-h$ & $\bullet$ & 0.3 \\
\hline
\end{tabular}

Tabla 2.2: Clases de patrones de SI. La letra $l$ corresponde a low (se afecta al parámetro por un factor de $60 \%$ por ejemplo) y la letra $h$ corresponde al $100 \%$ del valor (high). A su vez se indica la probabilidad de que un sujeto presente este tipo de clase 77 .

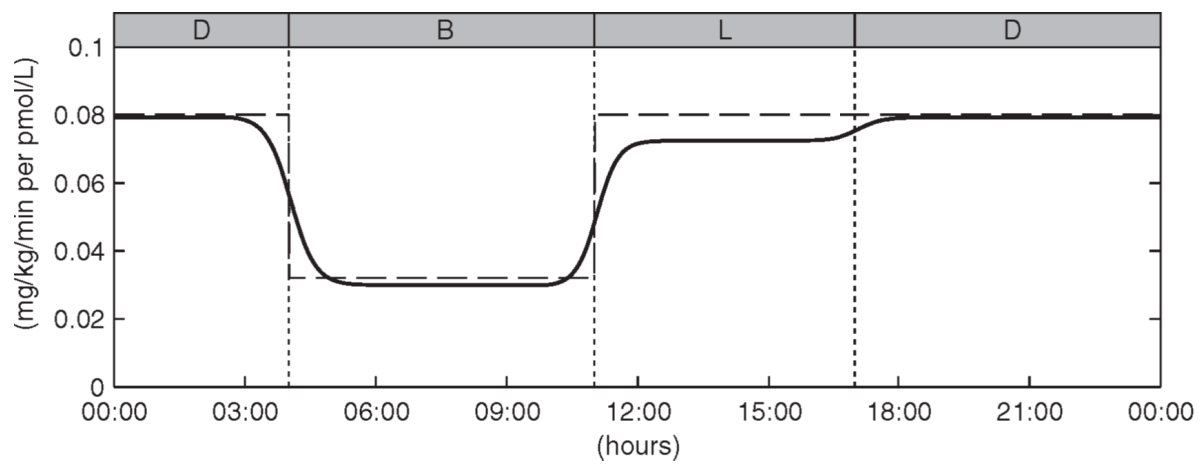

Figura 2.11: Perfiles de variación de SI para la clase virtual 5. Nominal (linea a trazos) y parámetro variante en el tiempo (línea sólida) 77].

\subsubsection{Absorción de carbohidratos y comidas mixtas}

La ensayos in-silico utilizados para el diseño y validación de estrategias de control de glucosa demandan la implementación de escenarios complejos para reflejar los desafíos del control glucémico. Anteriormente se mencionó la importancia de la representación realista de un modelo de absorción de glucosa. Los modelos presentados tanto por Hovorka et al. y Dalla Man et al. no tienen en cuenta la composición de la comida.

De acuerdo a la literatura $73,77,78,100$, la variación paramétrica obtenida en el modelo de la tasa de aparición de glucosa se atribuye principalmente a la composición de la comida. Los parámetros correspondientes al modelo de absorción fueron modificados para presentar un perfil variante en el tiempo entre comidas.

Tal como se explicó en la sección anterior, los parámetros de absorción de glucosa $k_{a b s}$, $k_{\min }, k_{\max }($ ec. 2.20 ) se vieron afectados por una clase variante en el tiempo, salvo que la probabilidad entre clases se distribuyó de forma uniforme. A su vez se afectó el el perfil de variación por un ruido aleatorio multiplicativo, utilizando las diferencias relativas medias de 78 y descritas por una distribución normal $N(\mu, \sigma)$, con $\mu=1,059$ y $\sigma=0,044$.

Para contar con perfiles de absorción más complejos y diversos, una biblioteca de perfiles de comidas mixtas fue agregada a la plataforma. Consiste en 60 curvas de comidas 
de diferente composición, cubriendo así una amplia variedad de perfiles de absorción de glucosa. Estas curvas fueron obtenidas por medio de estimación bayesiana de la tasa de aparición de glucosa a partir de estudios clínicos realizados en personas sin diabetes 100 . 101. Aún así, estos perfiles permiten ensayar tanto terapias a lazo abierto como a lazo cerrado ante perfiles de aparición de glucosa más desafiantes, ya que se consideran comidas de Alto Índice Glucémico (AIG) y de Bajo Índice Glucémico (BIG).

De acuerdo a la Federación Argentina de Diabetes (FAD), el índice glucémico es un método que sirve para evaluar y clasificar los alimentos que contienen hidratos de carbono según el impacto que tienen sobre la glucemia después de ser digeridos y absorbidos en el intestino. Un alimento de bajo índice glucémico produce una respuesta menor en la glucemia y más prolongada, lo que es beneficioso para el manejo de la diabetes. Un alimento de índice alto se absorbe rápidamente produciendo una glucemia elevada pero de corto tiempo. Esto es bueno como fuente de energía rápida para el ejercicio pero no para una persona con diabetes, salvo en el tratamiento de una hipoglucemia 102 .

Los factores que influencian el índice glucémico son varios:

1. el tipo de almidón o de azúcar simple que contiene: por ejemplo almidón de la papa, el azúcar común o refinada, en gaseosas o golosinas son de AIG; pero el tipo de azúcar que contienen las frutas o la leche no.

2. la presencia de grasa y de fibra en el alimento: es el caso de los hidratos de carbono presentes en las legumbres o las frutas secas que se encuentran junto con grasa y fibra soluble (AIG).

3. el tipo de procesado o de cocción: así es como el puré de papas tiene un índice glucémico mayor que la papa frita y el jugo de una fruta tiene un índice mayor que el de la fruta entera. El frío de los alimentos en la heladera reduce el índice glucémico.

En el apéndice A se describen la composición de las 60 comidas y su contenido de carbohidratos en la tabla A.1. En la plataforma de simulación, las tasas de aparición de glucosa de la biblioteca se modulan mediante un factor aleatorio (tanto en duración como en amplitud, sin afectar al área) y también se tiene en cuenta el peso corporal del sujeto, para aumentar la variabilidad de las respuestas entre los sujetos. Cuando se selecciona una comida mixta en la plataforma de simulación, el modelo de absorción es reemplazado por el perfil de absorción de la comida seleccionada.

\subsubsection{Comentarios finales}

La plataforma desarrollada permitió avanzar al grupo de trabajo en el desarrollo de estrategias de control de glucosa, tanto de lazo abierto como de lazo cerrado. Cabe destacar que para todas las publicaciones realizadas en esta tesis se utilizó la plataforma UNLP. Hoy en día es utilizada por diferentes miembros del grupo y otros colaboradores, como el grupo del Instituto Tecnológico de Buenos Aires (ITBA), para el avance en el desarrollo del algoritmo de control de lazo cerrado ARG [20]. El desarrollo de este algoritmo y los ensayos clínicos realizados se especificarán en el Capítulo 4 de esta tesis.

La plataforma se encuentra en constante desarrollo. Por ejemplo, se están considerando diferentes fuentes de representación de los efectos del ejercicio sobre la dinámica de la glucosa. Esto se desarrolla en el siguiente capítulo.

En las siguientes secciones de este capítulo, se describirán propuestas realizadas a partir de distintas colaboraciones con otros grupos de trabajo. Estas propuestas surgen gracias al desarrollo de la plataforma de simulación UNLP y del basto conocimiento y práctica de los modelos matemáticos de la dinámica de la glucosa en sujetos con DMT1. 


\subsection{Modelo orientado al control}

A continuación se presenta de forma breve el modelo orientado al control presentado por Colmegna et al. [103. Este modelo fue utilizado para el desarrollo de varias propuestas presentadas en esta tesis. En particular, se describe su formulación Linear Time Invariant (LTI).

Como se ha mencionado, los modelos matemáticos para simulación son de gran utilidad para realizar ensayos in-silico, permitiendo realizar pruebas seguras de estrategias de control glucémico. La dinámica de la GP puede ser descompuesta en diversos sub-sistemas y para cada uno se han propuesto modelos dinámicos [104]. Para el caso particular de diseño de controladores, es de interés representar la sola acción de la insulina sobre la GP con una formulación matemática más simple que los modelos utilizados para la simulación.

En particular, los modelos orientados al control se caracterizan por ser modelos paramétricos de bajo orden. Estos generalmente derivan de modelos fisiológicos utilizados para simulaciones, siendo algunos especialmente diseñados para ser usados en controladores de lazo cerrado [55]. La mayoría de los parámetros metabólicos relacionados al sistema glucosa-insulina no son fácilmente identificables en la práctica. Por esta razón, se recomienda sintonizar los modelos a partir de una pequeña cantidad de parámetros específicos del paciente de fácil obtención, permitiendo así su personalización [84].

El modelo diseñado en [103 corresponde a un modelo lineal de parámetros variantes Linear Parameter-Varying ( $(\overline{\mathrm{LPV}})$ ) obtenido a partir del simulador fisiológico UVA/Padova [75]. Este modelo de orden reducido demuestra ser adecuado para el diseño de controladores y su desempeño fue comparado con otros modelos de la literatura 67, 105. El modelo de bajo orden identificado queda expresado por la siguiente función de transferencia

$$
\frac{G(s)}{U(s)}=k \frac{(s+z)}{\left(s+p_{1}\right)\left(s+p_{2}\right)\left(s+p_{3}\right)} e^{-15 s}
$$

donde la entrada al sistema corresponde al suministro de insulina subcutánea en [pmol] y la salida es la concentración de glucosa en $[\mathrm{mg} / \mathrm{dl}]$. Este modelo puede individualizarse para cada paciente adulto del simulador UVA/Padova. El valor de la ganancia $k$ se ajusta considerando la regla ad-hoc "1800" (1800/TDI) [14], la cual relaciona la sensibilidad insulínica con la insulina total diaria (TDI, Total Daily Insulin) del sujeto. Desde el punto de vista médico, la regla de 1800 indica la caída máxima de la concentración de glucosa, medida en $\mathrm{mg} / \mathrm{dl}$, después de una inyección de $1 \mathrm{U}$ de insulina de acción rápida. La variación de la sensibilidad a la insulina a diferentes concentraciones de glucosa se representa mediante el polo dominante $p_{1}$. De esta manera, el ancho de banda (BW) del modelo fue diseñado para variar con el nivel de glucosa subcutánea $\mathrm{g}(\mathrm{t})[\mathrm{mg} / \mathrm{dl}]$.

Para simplificar el diseño de controladores, se realiza una implementación LTI de este modelo, como se lo ha hecho en trabajos anteriores [20]. Para obtener un modelo LTI, $p_{1}(g(t))$ se fija en $p_{1}\left(g_{b}\right)$, siendo $g_{b}$ la concentración basal de glucosa correspondiente. Teniendo en cuenta que la acción de control suele añadirse a la tasa de infusión de insulina basal $\left(i_{b}\right)$, el punto de operación del modelo LTI es $\left(i_{b} ; g_{b}\right)$.

Los parámetros identificados a partir del promedio de las respuestas en frecuencia de la cohorte de sujetos adultos virtuales son los siguientes: $k=-1,6788 \times 10^{-5}, z=0,1501$, $p_{1}=0,0035, p_{2}=0,0138$ у $p_{3}=0,0143$.

Tomando en cuenta la parte realizable de la ecuación (2.41), se puede obtener la realización en espacio de estados de la forma canónica observable:

$$
\begin{aligned}
\dot{x} & =A x(t)+B u_{\Delta}(t) \\
y_{\Delta} & =C x(t)
\end{aligned}
$$


con

$$
\begin{aligned}
& A=\left[\begin{array}{ccc}
0 & 1 & 0 \\
0 & 0 & 1 \\
-\alpha_{1} & -\alpha_{2} & -\alpha_{3}
\end{array}\right] \\
& B=k\left[\begin{array}{lll}
z & 1 & 0
\end{array}\right]^{T} \\
& C=\left[\begin{array}{lll}
1 & 0 & 0
\end{array}\right],
\end{aligned}
$$

donde $x(t)$ son los estados del sistema glucosa-insulina, $u_{\Delta}(t)$ e $y_{\Delta}$ son las desviaciones del punto de operación $\left(u_{o p} ; y_{o p}\right)$ y $\alpha_{i=1,2,3}$, corresponden a los coeficientes del polinomio característico del sistema (2.41), de forma tal que $\alpha_{1}=p_{1} p_{2} p_{3}, \alpha_{2}=\left(p_{2} p_{3}+p_{1} p_{2}+p_{1} p_{3}\right)$, $\alpha_{3}=\left(p_{1}+p_{2}+p_{3}\right)$ respectivamente.

\subsection{Modelo LPV considerando variación intra-paciente}

La implementación de los perfiles de variación intra-paciente (sección 2.2.2) en la plataforma de simulación UNLP permitió formular una ampliación del modelo de orden reducido orientado al control presentado en 103. En colaboración con la Dr. Moscoso-Vasquez del ITBA y el grupo trabajo del ITBA, se presentó un modelo LPV que contempla la variación intra-paciente. El desarrollo completo de este modelo se publicó en [106].

Esta propuesta consiste en la obtención de un modelo de bajo orden diseñado para la regulación de la glucosa en la DMT1 a partir de los datos metabólicos del simulador UVA/Padova. Este modelo capta no sólo el estado no lineal del sistema de insulina, sino también las variaciones relacionadas con los cambios diarios de la SI. Para superar la gran variabilidad entre sujetos, el modelo también puede ser personalizado en base a la información del paciente de forma a priori. La estructura está preparada para el diseño de controladores LPV y representa la dinámica desde la entrada de insulina subcutánea hasta la salida de glucosa subcutánea.

Siguiendo un procedimiento similar al presentado en [103] para la obtención del modelo $\mathrm{LPV}_{g}$, se desarrolla una extensión del modelo presentado en (2.41) que incluye variabilidad intra-paciente, denominada modelo $\mathrm{LPV}_{i}$. Las linealizaciones del modelo UVA/Padova se obtienen para cada adulto in-silico de la versión de distribución, desde la administración de insulina subcutánea ( $\mathrm{pmol} / \mathrm{min}$ ) hasta la desviación de la concentración de glucosa subcutánea $(\mathrm{mg} / \mathrm{dl})$ a diferentes concentraciones de glucosa $g$ en estado estacionario y a valores del Factor de Variación de la Sensibilidad a la Insulina $\left(S_{I, V F}\right)$. Para ello, se calculó para cada sujeto y cada $g$ y $S_{I, V F}$, la tasa de infusión de insulina basal necesaria para mantener el nivel de glucosa constante. Para cubrir completamente todos los escenarios discutidos en [77, 107], los parámetros $V_{m x}$ y $k_{p 3}$ del modelo son modulados por $S_{I, V F}$ dentro del rango $[0,4 ; 1,7]$. Se define $S_{I, V F}=S_{I} / S_{I, n o m}$, donde $S_{I, n o m}$ representa los valores nominales de $V_{m x}$ y $k_{p 3}$ del modelo.

Teniendo en cuenta que la ganancia continua del modelo 2.41 es $\frac{k z}{p_{1} p_{2} p_{3}}$ y que el ancho de banda es independiente de $k$, se propone una extensión al modelo $\mathrm{LPV}_{g}$ haciendo que el parámetro $k$ dependa tanto de $g$ como de $S_{I, V F}$ como se muestra en la Fig. 2.12.

De esta manera, la ganancia $k$ se utiliza para compensar tanto las variaciones interpacientes por medio de la regla "1800" como las variaciones intra-pacientes haciendo un cambio de $k$ de acuerdo al perfil de SI. Este último podría ser un perfil general como los presentados en la sección 2.2.2 o un perfil obtenido a partir de datos clínicos.

La eficacia de este modelo se evalúa en comparación con el modelo anterior $\left(\mathrm{LPV}_{g}\right)$. Ambos modelos se comparan en términos de sus diferencias con respecto al modelo UVA/Padova a lazo abierto y cerrado. En la Figura 2.13 se muestran los valores medios de Relative Mean 


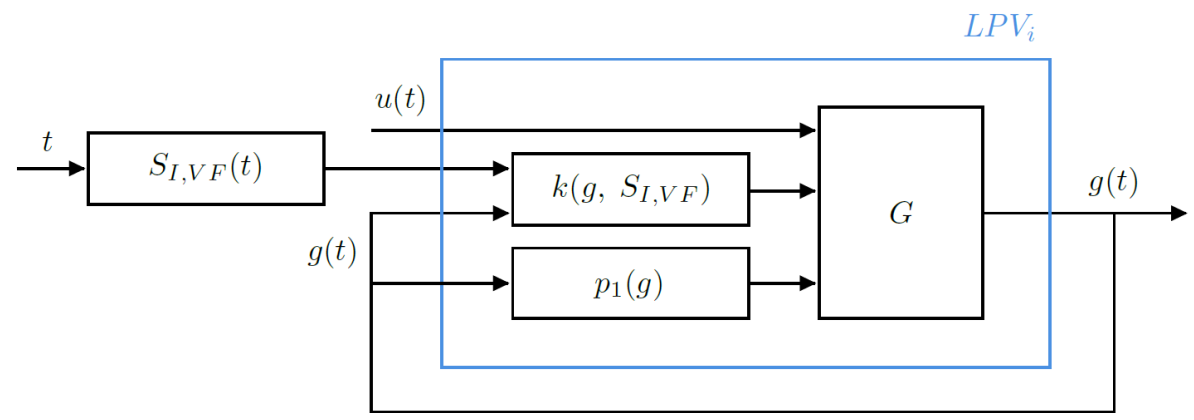

Figura 2.12: Estructura del modelo $\mathrm{LPV}_{i}$ promedio.

Square Error (RMSE) para los 10 adultos in-silico a diferentes valores de $g$ y $S_{I, V F}$. Nótese que se puede obtener un RMSE más bajo con el modelo $\mathrm{LPV}_{i}$ que con el $\mathrm{LPV}_{g}$ para la mayoría de las concentraciones de glucosa. El modelo propuesto supera a los anteriores modelos orientados al control de DMT1, lo que podría dar lugar a controladores más robustos y fiables para la regulación de glucosa.

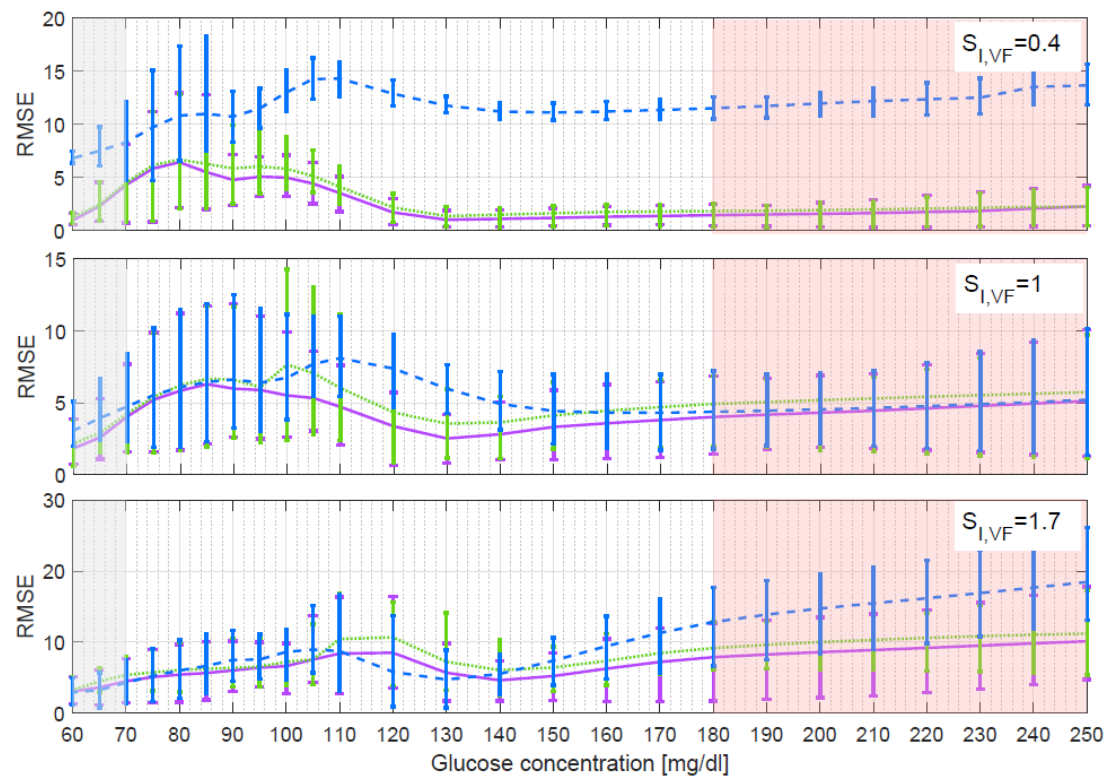

Figura 2.13: RMSE promedio entre las respuestas temporales del modelo $\mathrm{LPV}_{g}$ personalizado (línea a rayas azul), $\mathrm{LPV}_{i}$ promedio (línea punteada verde) y LPVi personalizado (línea sólida violeta), comparados con el modelo no-lineal del UVA/Padova para bolos de $1 \mathrm{U}$ para diferentes valores de $S_{I, V F}$. Arriba: caso más resistente $\left(S_{I, V F}=0,4\right)$; medio: caso nominal $\left(S_{I, V F}=1\right)$; abajo: caso más sensible $\left(S_{I, V F}=1,7\right)$. Las barras verticales indican valores máximos y mínimos obtenidos.

\subsection{Plataforma de simulación intervalar}

A partir de una estancia de investigación realizada en la ciudad de Bogotá, donde se colaboró junto a los doctores Fabian León-Vargas (Universidad Antonio Nariño) y Maira García-Jaramillo (Universidad EAN), se desarrolló una plataforma de simulación basada en la aritmética intervalar con el objetivo de poder predecir los peores casos posibles, tanto de hiper- como de hipoglucemia. Este simulador fue presentado y publicado en [108].

Como se presentó en la sección 2.1. hoy en día se cuenta con diversos modelos de los compartimentos del sistema glucosa-insulina. En el trabajo 91 se revisaron varios de estos modelos en consideración en su habilidad de predecir la excursión de glucosa 
ante incertidumbre y variación intra-paciente. Como resultado, se obtuvieron versiones de los modelos implementando análisis intervalar. El esquema de simulador que se propone puede visualizarse en la Fig. 2.14. En este esquema se representa la incertidumbre mediante modelos intervalares.

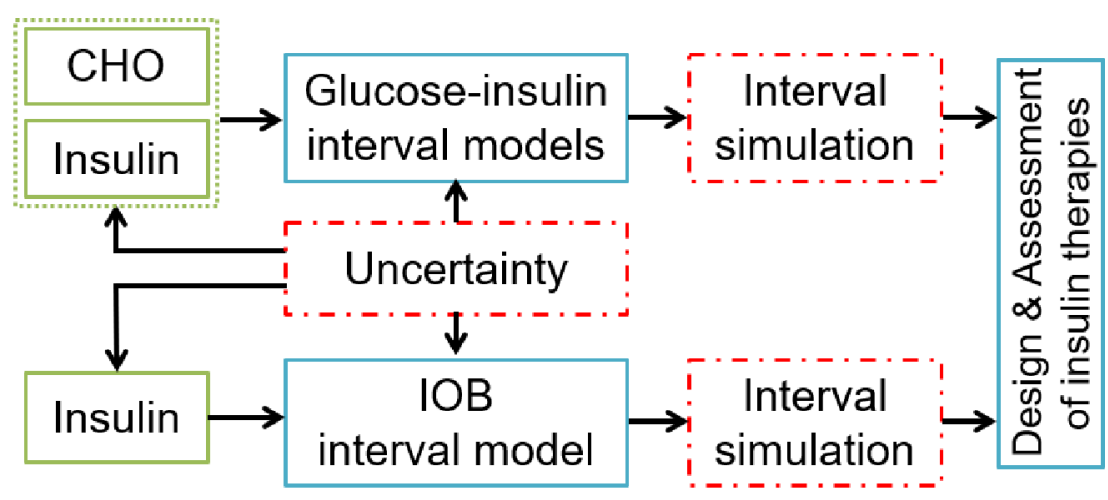

Figura 2.14: Esquema de simulador intervalar. La incertidumbre afecta a diferentes componentes relacionados a la dinámica glucosa-insulina, como por ejemplo las entradas de insulina o carbohidratos, parámetros del modelo, o la estimación de IOB.

En este esquema, la incertidumbre está representada por modelos intervalares cuyos parámetros, entradas y/o estados iniciales pueden tomar valores de intervalo. Con el fin de obtener un cálculo exacto del intervalo, cada modelo incluido aquí fue analizado y puesto en su forma óptima usando la teoría de análisis intervalar modal (Modal Interval Analysis, MIA) [109. MIA se utiliza para reducir el impacto de múltiples instancias de la misma variable en la expresión a evaluar, evitando la sobre-estimación de la simulación del intervalo. Se han estudiado dos modelos adicionales a los presentados en trabajos anteriores 109 111. Estos modelos adicionales son: el modelo de orden reducido orientado al control de Colmegna et al. [103]; y el modelo de estimación de insulina a bordo de LeonVargas et al. [96]. A este último, se lo utilizará y detallará más adelante en el siguiente capítulo de la tesis.

Cada modelo fue analizado con respecto a sus parámetros, entradas y condiciones iniciales teniendo en cuenta incertidumbre, y reescrito de tal manera que se obtiene el rango exacto. En los casos en que se requirió, el teorema de coerción para la optimización se aplicó, teniendo en cuenta la definición de un operador dual como Dual $\left(\left[a_{1}, a_{2}\right]\right)=\left[a_{2}, a_{1}\right]$ para obtener un cálculo racional óptimo (CRO). El lector puede encontrar detalles sobre este procedimiento utilizando MIA en 109,112 .

En este caso, el modelo de Colmegna et al. fue estudiado considerando la variabilidad intra-paciente y las diferentes fuentes de incertidumbre, por ejemplo, en la ingesta de alimentos, la dosis de insulina, la glucosa en sangre preprandial, la sensibilidad a la insulina y los estados iniciales. Los parámetros y los estados iniciales relacionados con estas incertidumbres se evaluaron utilizando MIA para obtener un CRO. El modelo obtenido es el 
siguiente:

$$
\begin{aligned}
x_{1}(t+1) & =\Delta t x_{2}(t)+x_{1}(t) \\
x_{2}(t+1) & =\Delta t x_{3}(t)+x_{2}(t) \\
x_{3}(t+1) & =\Delta t\left(p_{1}\left(-p_{2} p_{3} \operatorname{Dual}\left(x_{1}(t)\right)-\left(p_{2}+p_{3}\right)+\operatorname{Dual}\left(x_{2}(t)\right)-\operatorname{Dual}\left(x_{3}(t)\right)\right)-\right. \\
& \left.-p_{2} p_{3} \operatorname{Dual}\left(x_{2}(t)\right)-\left(p_{2}+p_{3}\right) \operatorname{Dual}\left(x_{3}(t)\right)\right)+\left(x_{3}(t)\right)+ \\
& +\Delta t(\operatorname{Basal}+\operatorname{Bolus})) \\
x_{4}(t+1) & =x_{4}(t)-\frac{\Delta t \operatorname{Dual}\left(x_{4}(t)\right)}{t_{\max , G}}+\Delta t D \\
x_{5}(t+1) & =x_{5}(t)+\frac{\left(x_{4}(t)-\operatorname{Dual}\left(x_{5}(t)\right)\right) \Delta t}{t_{\max , G}} \\
G_{e}(t) & =k\left(z x_{1}(t)+x_{2}(t)\right)+\frac{0,8 x_{5}(t)}{t_{\text {max }, G} k_{r a}}
\end{aligned}
$$

donde $p_{1}, p_{2}$ y $p_{3}$ y $z$ son parámetros que corresponden a los tres polos y el cero, respectivamente, de la representación de Laplace del modelo de Colmegna et al. [113] (ec. (2.41)). Basal y Bolus (IU) corresponden a entradas relacionadas con la administración de insulina. El modelo se amplió considerando un modelo de absorción de glucosa, donde los estados $x_{4}$ y $x_{5}$ se adaptaron del modelo de Hovorka et al. [69 para estimar la tasa de absorción de glucosa (ec. (2.11)). La entrada $D(\mathrm{mg})$ está relacionada con la cantidad de CHO ingerida, $t_{\max , G}(\mathrm{~min})$ es el tiempo de aparición máxima de la glucosa en plasma, y $k_{r a}$ (adimensional) es la biodisponibilidad de los hidratos de carbono. Finalmente, la estimación de la glucosa en plasma viene dada por $G_{e}(t)$. El paso de tiempo $\Delta t$ se define de tal forma que $\Delta t<1 /\left(p_{1}+p_{2}+p_{3}\right)$ satisfaga las condiciones de monotonicidad requeridas.

$\mathrm{El}$ simulador intervalar fue implementado usando el software Matlab. Una clase llamada aritmética de intervalos vectoriales [114], que incorpora la llamada aritmética de intervalo extendido (aritmética Kaucher) y permite utilizar la teoría MIA, se utilizó aquí para obtener la envolvente de la glucosa (es decir, el rango de las trayectorias) para los modelos de intervalo.

La plataforma permite seleccionar: el modelo de cada subsistema, el (\%) de incerti-

\begin{tabular}{|c|c|c|}
\hline Subsistema & Modelo & Parámetros \\
\hline $\begin{array}{l}\text { Absorición de } \\
\text { carbohidratos }\end{array}$ & $\begin{array}{l}\text { Hovorka et al. (2004) } 69 \\
\text { Dalla Man et al. (2007) } 73\end{array}$ & $\begin{array}{l}D, A_{G}, t_{\max , G} \\
D\end{array}$ \\
\hline $\begin{array}{l}\text { Absorción de } \\
\text { insulina subcutánea }\end{array}$ & $\begin{array}{l}\text { Hovorka et al. (2004) } 69 \\
\text { Dalla Man et al. (2007) } 73\end{array}$ & $\begin{array}{l}u(t) \\
u(t)\end{array}$ \\
\hline $\begin{array}{l}\text { Acción de la insulina y } \\
\text { dinámica de la glucosa }\end{array}$ & $\begin{array}{l}\text { Bergman et al. (1981) } 64 \\
\text { Colmegna et al. }(2018) \quad 103\end{array}$ & $\begin{array}{l}p_{1}, p_{2}, p_{3}, p_{4}, n \\
D, \text { Basal }, \text { Bolus }, p_{1}, k\end{array}$ \\
\hline Estimación de IOB & León-Vargas et al. (2013) $[96]$ & $K_{D I A}$ \\
\hline
\end{tabular}
dumbre asociada a cada parámetro o entrada, el sujeto virtual, el escenario de comidas e insulina, y las salidas a graficar. Es importante destacar que el uso de la interfaz de usuario es opcional, el escenario de simulación puede ser configurado directamente en un archivo de texto. Los parámetros y las entradas consideradas inciertas en cada uno de los modelos de intervalo incluidos en la simulación de intervalos se presentan en la tabla 2.3 .

Tabla 2.3: Parámetros de los modelos de cada subsistema a los que se les adjudicó incertidumbre en el simulador intervalar.

Las excursiones de glucosa intervalar obtenidas de la combinación de diferentes modelos correspondientes a varios subsistemas se muestran en la Fig. 2.15 para dos escenarios 
de prueba. En este caso, se utilizaron los modelos de tasa de aparición de la glucosa y de absorción de insulina de Dalla Man et al. A su vez, se consideraron los modelos de acción de la insulina y la dinámica de la glucosa de Bergman et al.. Así se demuestra la funcionalidad que posee el simulador intervalar de interactuar con los modelos dinámicos de glucosa-insulina de diferentes autores. Los modelos de intervalos implementados se pueden encontrar en [110].

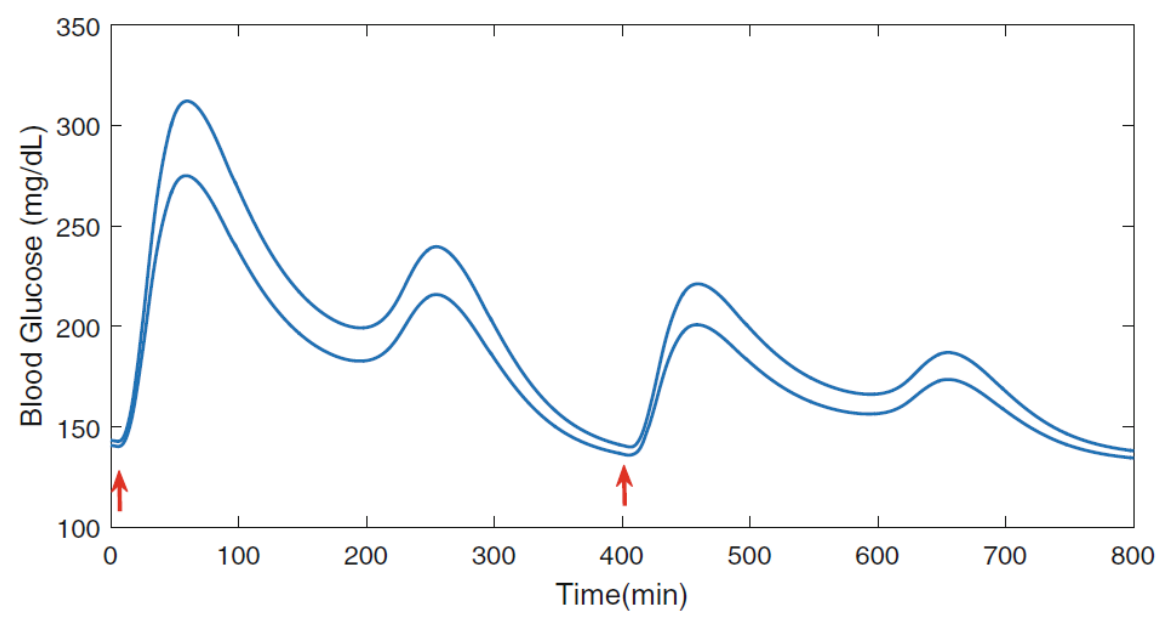

Figura 2.15: Envolvente de la excursión de glucosa obtenida del sujeto virtual \#5 (ajustado del simulador UVA/Padova) considerando dos comidas de $100 \mathrm{~g}$ y $50 \mathrm{~g}$ respectivamente con $10 \%$ de incertidumbre. Las flechas rojas indican el tiempo de la comida. Dos bolos (6 y 5 U) fueron suministrados a la hora de la comida. Una incertidumbre del $3 \%$ y $2 \%$ se tuvo en cuenta en los parámetros $p_{1}$ y $p_{2}$ respectivamente (del modelo de Bergman), para tener en cuenta una variación de la SI.

El simulador intervalar presenta dos características a destacar. La primera, como se acaba de remarcar, está relacionada con el uso de modelos de diferentes autores para estimar la salida de cada subsistema de la dinámica glucosa-insulina, que según lo relevado en la literatura, no existen simuladores del sistema glucosa-insulina que permitan esta funcionalidad, ya sea con fines de diseño o de prueba. La integración de estos modelos se logró aquí mediante la homogeneización de las unidades de las señales de entrada y salida según lo requerido por la combinación de modelos correspondiente. Esta característica permite una dinámica más compleja para un sujeto virtual en particular, que puede ser utilizada, por ejemplo, para el diseño o la evaluación de sistemas de control de lazo cerrado en el contexto del $\mathrm{PA}$, donde es importante obtener un buen rendimiento del controlador antes de proceder con los ensayos clínicos. Adicionalmente, se ha incluido un conjunto de sujetos virtuales del simulador de UVA/Padova ajustado en [110].

La segunda característica está relacionada con las diferentes fuentes de incertidumbre (parámetros, condiciones iniciales y entradas) que pueden asignarse a los modelos de intervalos incluidos en este simulador. La variabilidad de la glucosa se percibe en realidad como el desafío más difícil de considerar en el diseño del control de la glucosa para la vida diaria de las personas con DMT1 [115]. Esta variabilidad fisiológica intra-paciente puede explicarse a partir de una serie de retos que aún quedan por afrontar, incluyendo el efecto del ejercicio, la enfermedad concurrente, las comidas con grandes cantidades de carbohidratos y la farmacocinética de la insulina subcutánea. La simulación de modelos intervalares con incertidumbre produce límites superiores e inferiores, en lugar de una respuesta única, definiendo una envolvente que incluye todas las posibles excursiones de la glucosa, dentro de las cuales se podría encontrar el comportamiento real del suejeto.

La incertidumbre integrada en la simulación, representada por la envolvente de salida, puede utilizarse para un análisis del peor caso, lo cual es extremadamente importante en el contexto de la diabetes. Por ejemplo, la predicción de posibles episodios de hipo o hiper- 
glucemia y el ajuste de la terapia de insulina en consecuencia. Es importante comprender que la simulación de intervalos puede usarse para apoyar el diseño de nuevas estrategias de control de la glucosa, ya que ofrece una estimación eficiente de todas las posibles dinámicas de respuesta que permiten anticipar los escenarios de riesgo para el paciente.

\subsection{Conclusiones}

En este capítulo se presentaron propuestas involucradas con el modelado y la simulación del sistema glucosa-insulina.

- Se presentaron los principales modelos desarrollados en la literatura y se compararon a fin de resaltar sus ventajas y debilidades.

- Se desarrolló una plataforma de simulación propia para el ensayo y validación de forma in-silico de estrategias de control de glucosa. La misma resulta versátil y permite diferentes formas de representar la variabilidad y problemáticas que presenta el control de glucosa.

- Se presenta un modelo orientado al control que incluya la variabilidad intra-paciente, para así permitir el desarrollo de controladores de lazo cerrado más robustos.

- Se presenta una plataforma de simulación que considera la aritmética intervalar

Gracias a los aportes realizados en este capítulo, se pudo desarrollar las estrategias de control glucémico presentadas a lo largo de esta tesis. 


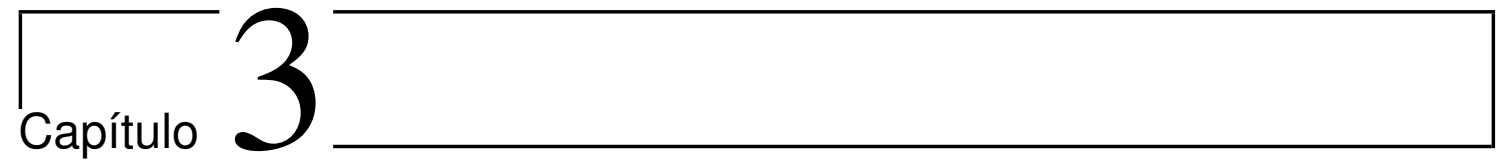

\section{Estrategias de control a Lazo-Abierto vía limitación de IOB}

En este capítulo se hará foco en el tratamiento diario a lazo abierto de las personas con DMT1. Aunque la visión del sistema de Páncreas Artificial está instaurada como el gran objetivo final en la mente de los investigadores, médicos y personas con diabetes desde hace décadas, aún hay mucho camino que recorrer para poder concebir un sistema de control de glucosa automático de lazo cerrado. Incluso hoy en día gran parte de las personas con DMT1 no dispone de las tecnologías pertinentes para un tratamiento de lazo cerrado. A su vez, no toda persona con diabetes busca un control de lazo cerrado, ya sea por gusto, o el hecho de no querer contar con tanta tecnología, por lo que disponer alternativas para el tratamiento cotidiano a lazo abierto aún es de suma importancia.

Uno de los mayores inconvenientes en el control de glucosa surge en las restricciones en la acción de control. El suministro de insulina no se produce por medio de la vía intravenosa sino de forma subcutánea. Esto impone una nueva dinámica a la acción de control debido al proceso de absorción de insulina desde el espacio subcutáneo hasta la concentración plasmática. El hecho de tener en cuenta la acumulación de insulina en el espacio subcutáneo ha sido de gran importancia en cuanto a la seguridad de los tratamientos. Poder estimar y regular cuanta insulina se inyecta al cuerpo resulta primordial para evitar hipoglucemias por la sobreactuación. En este Capítulo se realizan dos propuestas para el control a lazo abierto de la glucosa para el tratamiento de la DMT1: una para el control posprandial y otra para el control durante el ejercicio. Ambas propuestas se basan en la estimación de los perfiles de la insulina a bordo (IOB).

En primer lugar, se introducen los diferentes métodos que se utilizan para estimar la IOB. Luego se introduce una estrategia para la compensación de comidas, en particular aquellas con alto índice glucémico, el super-bolo automático. Esta estrategia fue presentada en el congreso internacional Advanced Technologies and Treatments of Diabetes (ATTD) 2016 [116] y luego publicado en una revista de alto impacto en 2018 [117]. Por último, se realiza una propuesta para proteger al sujeto durante la actividad física, tanto para evitar una posible hipoglucemia durante el ejercicio, como para evitar un rebote hiperglucémico luego del mismo. Esta propuesta fue presentada en el congreso RPIC XVIII en su edición 2019 [118 y en el congreso internacional ATTD 2019 [119].

\footnotetext{
${ }^{1} \mathrm{El}$ congreso anual internacional ATTD, establecido en el año 2008, es donde se presentan las tecnologías más innovadoras para el tratamiento de la diabetes.
} 


\subsection{Estimación de IOB}

La estimación de la IOB es empleada por algunas bombas de insulina para prevenir el exceso de insulina acumulada en el cuerpo, particularmente cuando se suministran bolos cercanos entre sí. Como se definió previamente en el Capítulo 1 Sección 1.2.2, el nivel de IOB se tiene en cuenta para el cálculo de los bolos (ec. 1.1). La individualización de la estimación de la IOB se suele hacer mediante la duración de la acción de la insulina, determinada por el parámetro DIA. Este parámetro suele definirse por un médico y puede configurarse tanto en la bomba (si el modelo lo permite) como en aplicaciones de cálculo de bolos [14]. En la Figura 3.1 se puede ver una representación simplificada de los perfiles de absorción para diferentes tipos de insulina, los cuales se han especificado en el Capítulo 1 Sección 1.2 .2 .

La estimación de la IOB se realiza mediante modelos simples y discretos como los implementados en las bombas de insulina actuales (basadas en líneas rectas o curvas), o mediante un modelo continuo de absorción subcutánea como los disponibles en la literatura 120,121. Cada uno de estos modelos se describe a continuación.

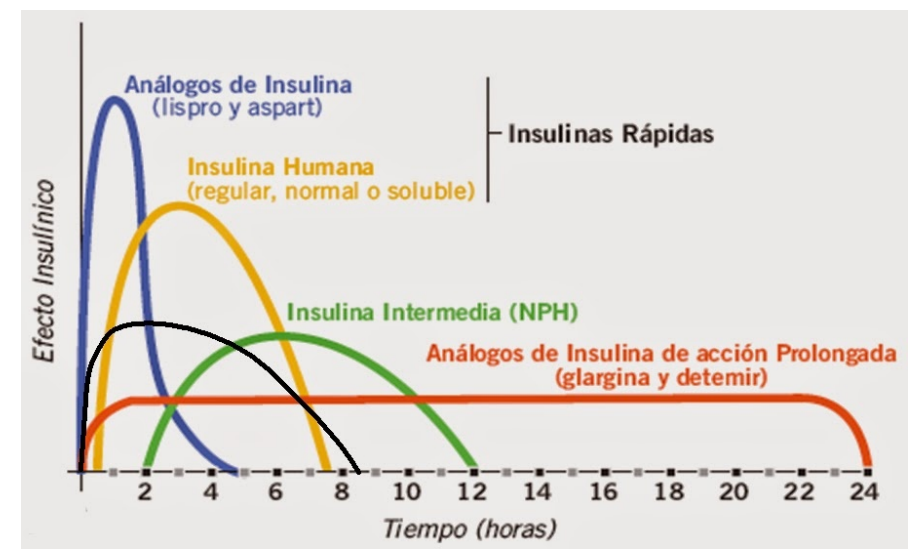

Figura 3.1: Representación de los diferentes tipos de insulina y sus curvas de absorción aproximadas.

\section{Estimación mediante rectas}

Algunas bombas utilizan gráficos lineales para hacer que el concepto de IOB sea más fácil de entender para los usuarios de calculadoras de bolos y sujetos con diabetes. El modelo simplemente describe la IOB como un recta de la forma

$$
\operatorname{IOB}\left(T_{k}\right)=I_{\text {bolus }} \cdot\left(1-\frac{\left(T_{k}-T_{\text {bolus }}\right)}{D I A}\right),
$$

donde $I_{\text {bolus }}$ es la cantidad total de insulina suministrada en un bolo anterior, $T_{k}$ es el tiempo actual en minutos, $T_{\text {bolus }}$ es el tiempo en el que se suministró el bolo, $T=T_{k}-T_{\text {bolus }}$ es el tiempo transcurrido desde el bolo anterior, y DIA es la duración de la acción de la insulina en horas.

Aunque este método resulta ser el más simple, el problema con este modelo es que se estima el bolo a bordo (Bolus On Board (BOB $)$ ) en lugar de IOB, ya que calcula la absorción de insulina a partir de inyecciones discretas de insulina (bolos). Además, es el modelo con mayor error con respecto al perfil de IOB real, ya que asume un perfil de línea recta no realista.

\section{Estimación mediante curvas}


Una ligera mejora del modelo anterior implementado en algunas bombas de insulina se basa en las curvas de absorción de insulina, a partir de las cuales se calcula el IOB como

$$
\operatorname{IOB}\left(T_{k}\right)=I O B \text { curve }{ }^{\top} \cdot I_{\text {past }}\left(T_{k-1}, T_{k-N}\right),
$$

donde IOBcurve ${ }^{\top}$ es un vector fila con $N$ elementos (factores menores o iguales a uno) representando la curva de acción de insulina, $I_{p a s t}\left(T_{k-1}, T_{k-N}\right)$ es un vector columna con un registro de la insulina suministrada en las últimas horas en un intervalo dado por el DIA, entre el tiempo $T_{k-N}$ hasta el instante $T_{k-1}$, y $N=$ floor $\left(D I A \cdot 60 / T_{s}\right)$, donde $T_{s}$ es el periodo de trabajo de la bomba en minutos.

\section{Estimación mediante modelo dinámico}

Un modelo dinámico lineal de dos compartimentos para representar la absorción subcutánea de insulina se utiliza en la mayoría de los ensayos in-silico y en la validación de estrategias de control (e.g. [70]). De hecho, esta representación dinámica se utiliza para el modelo de absorción de insulina subcutánea en el simulador UVA/Padova T1DM, que se describe ampliamente en la literatura [122], dada por:

$$
\begin{gathered}
\left\{\begin{array}{c}
\dot{I}_{s c 1}=-\left(k_{d}+k_{a 1}\right) I_{s c 1}+u \\
\dot{I}_{s c 2}=k_{d} I_{s c 1}-k_{a 2} I_{s c 2}
\end{array}\right. \\
I O B=I_{s c 1}+I_{s c 2},
\end{gathered}
$$

donde $I_{s c 1}$ y $I_{s c 2}$ son, respectivamente, la cantidad de insulina monomérica y no-monomérica en el espacio subcutáneo, $u$ es la tasa de infusión exógena de insulina en [pmol $/ \mathrm{min} / \mathrm{kg}$ ], $k_{d}\left[\min ^{-1}\right]$ es la tasa de disociación de insulina, y $k_{a 1}\left[\mathrm{~min}^{-1}\right]$ y $k_{a 2}\left[\mathrm{~min}^{-1}\right]$ son las constantes de tasa de absorción. Este modelo es el más exacto y realista de los presentados, y a su vez permite una estimación continua del IOB a partir de la entrada de insulina.

Con el fin de minimizar los parámetros a ajustar, se considera un modelo que puede personalizarse basándose únicamente en la información clínica a-priori, como el modelo dinámico que se presenta en [120] y en [96]:

$$
\begin{aligned}
& \left\{\begin{array}{l}
\dot{I}_{s c 1}=-K_{\mathrm{DIA}} I_{s c 1}+u \\
\dot{I}_{s c 2}=K_{\mathrm{DIA}}\left(I_{s c 1}-I_{s c 2}\right)
\end{array}\right. \\
& I O B=I_{s c 1}+I_{s c 2} .
\end{aligned}
$$

En este caso, un único parámetro $K_{\text {DIA }}\left[\mathrm{min}^{-1}\right]$ tiene que ser ajustado para representar el DIA del sujeto. En la tabla 3.1 se muestran los valores de $K_{\text {DIA }}$ correspondiente a diferentes valores de DIA.

Tabla 3.1: Parámetro $K_{\text {DIA }}\left(\min ^{-1}\right)$ del modelo de IOB 3.5 para diferentes duraciones de acción de insulina.

\begin{tabular}{cccccccc}
\hline$D I A(\mathrm{~h})$ & 2 & 3 & 4 & 5 & 6 & 7 & 8 \\
\hline$K_{\text {DIA }} \times 10^{-3}$ & 39 & 26 & 19.5 & 16.3 & 13 & 11.3 & 9.9 \\
\hline
\end{tabular}

Para la aplicación de la estimación de IOB en dispositivos de dosificación de insulina es importante tener en cuenta su representación en espacio de estados discretos, que resulta en:

$$
\begin{aligned}
x(k+1) & =\left[\begin{array}{cc}
1-K_{\mathrm{DIA}} T_{r} & 0 \\
K_{\mathrm{DIA}} T_{r} & 1-K_{\mathrm{DIA}} T_{r}
\end{array}\right] x(k)+\left[\begin{array}{c}
T_{r} \\
0
\end{array}\right] u(k) \\
\operatorname{IOB}(k) & =\left[\begin{array}{ll}
1 & 1
\end{array}\right] x(k)
\end{aligned}
$$


siendo $T_{r}$ el tiempo de muestreo en minutos. Notar que $T_{r}$ puede ser mucho menor que el tiempo de muestreo de la bomba o del controlador dado que el algoritmo en donde se implementa la estimación puede programarse enteramente en software. El modelo dado por (3.7) será utilizado para el desarrollo de las propuestas de este capítulo.

La estimación de IOB resulta muy importante para la prevención de hipoglucemias, tanto en el cálculo manual del bolo (1.1) como en el desarrollo de técnicas de dosificación de insulina a lazo abierto y a lazo cerrado. Aún así, existe una confusión generalizada entre los médicos y los pacientes con respecto a la selección de un DIA adecuado. Muchos especialistas han destacado recientemente los problemas que surgen cuando se establece un DIA inapropiadamente corto. Por otro lado, algunos sistemas no permiten seleccionar un DIA inferior a 5 horas a sus usuarios [123. Dado que la dinámica de absorción de insulina subcutánea puede variar con el tiempo en los sujetos, especialmente si el Índice de Masa Corporal (IMC) cambia, es importante actualizar el DIA regularmente. Por estos motivos, se han propuesto protocolos de investigación para medir con precisión el DIA y se reconoce la gran importancia de que los fabricantes de insulina verifiquen un DIA representativo de las insulinas de acción rápida en el mercado [124].

Estimadores desarrollados recientemente basados en lecturas deCGM 125, 126 podrían emplearse para la estimación de IOB o de parámetros implicados en la dinámica de absorción subcutánea de insulina. Estos observadores, comúnmente empleados en sistemas de control de lazo cerrado, realizan una estimación en tiempo real de la concentración de insulina en plasma, lo que permitiría obtener un valor más preciso del DIA del paciente.

\subsection{Control glucémico posprandial}

Las bombas de insulina actuales incorporan asesores de bolo que ayudan a los usuarios a calcular los bolos prandiales, un ajuste de insulina basal personalizable para hacer frente a los cambios de sensibilidad diarios, alarmas preventivas, cantidad de carbohidratos en la dieta y cantidad estimada de IOB [14, 127]. Aunque el tratamiento CSII proporciona un mejor control glucémico que las terapias convencionales con MDI y reduce el riesgo de hipoglucemia, la mayoría de las personas con DMT1 no mantienen su glucemia en rango. Recientemente, el primer producto comercial mundial para el control híbrido de la glucosa ha sido lanzado en el mercado de EE.UU. (MiniMed 670G system, Medtronic Inc., Northridge $\mathrm{CA}$ ). Aunque representa un gran avance hacia el control totalmente automático, la acción feedfoward para el control posprandial -correspondiente a los bolos de insulina- todavía debe realizarse manualmente. Las altas excursiones hiperglucémicas después de las comidas y la hipoglucemia postabsortiva tardía siguen constituyendo un desafío terapéutico 128. Para evitar estos síntomas, algunas personas con DMT1 confían en la rutina de la suspensión de insulina basal durante varias horas, dejando que el bolo de la comida cubra las necesidades de insulina tanto prandial como basal [129]. Además, hay tanto ensayos comparativos in-silico [130] y clínicos [131] donde muestran un mejor control de la glucosa posprandial usando otras combinaciones de basal-bolo que la estándar.

Cuando la administración de insulina basal se detiene o se reduce durante un período de tiempo y la cantidad de insulina basal que no se administra se añade a un bolo de comida o corrección, se crea un super-bolo. Este cambio de insulina basal a forma de bolo crea uno de mayor tamaño, el cual es particularmente beneficioso cuando la insulina se necesita rápidamente. Estas situaciones pueden incluir, pero no se limitan, la necesidad de cubrir alimentos de alto índice glucémico o grandes consumos de carbohidratos, requiriéndose de un rápido retorno a la normoglucemia desde un nivel elevado de azúcar en la sangre 14. Hoy en día, los usuarios de bombas suelen establecer manualmente un tratamiento de Super-Bolo $(\underline{\mathrm{SB}})$ para algunos tipos de comidas de acuerdo con la recomendación del médico [14. Sin embargo, debe suprimirse una cantidad de insulina basal, que no siempre 
se adivina correctamente. De hecho, una de las principales preguntas abiertas en los foros de diabetes sobre el $[\mathrm{SB}$ es "¿cuánto tiempo puede reducirse o detenerse el suministro basal antes de que se produzca un rebote en la glucosa una vez que el SB se haya absorbido?" En las bombas de insulina comerciales actuales todavía no se ha implementado un SB automático, probablemente debido a esta pregunta abierta. El cálculo automático de un SB puede ser abordado de diferentes maneras, desde la forma más simple de definir un tiempo fijo independientemente del sujeto, hasta formas más complejas que implican información clínica del usuario, como se propone a continuación.

En esta sección se presenta una metodología que tiene como objetivo mejorar la administración de los bolos de insulina para lograr un mejor equilibrio entre el rendimiento y la seguridad en las terapias de lazo abierto. Este enfoque permite mejorar las actuales terapias de control de la glucosa, así como conocer las implicaciones de la restricción de la IOB en los tratamientos usuales. En particular, un algoritmo que da forma automáticamente a un $\mathrm{SB}$ sin necesidad de intervención manual en su cálculo. La idea que subyace a la propuesta proviene de la metodología registrada en las solicitudes de patentes y prioridades [132 y [133. Esta idea consiste en imponer una restricción sobre el perfil de IOB generado por el tratamiento.

\subsubsection{Marco teórico}

La motivación principal para implementar un tratamiento en base al SB para el manejo de la glucosa es compensar la dinámica lenta de la acción de la insulina con respecto al tiempo en que las comidas elevan la glucosa en la sangre.

El sistema glucosa-insulina es un sistema dinámico positivo, es decir, un sistema dinámico donde todas las variables de estado y las entradas son positivas. El trabajo 134 presenta algunos resultados relevantes sobre este tipo de sistemas sometidos a perturbaciones. Una situación crítica aparece cuando la respuesta a un pulso de perturbación alcanza un pico más rápido que la respuesta a un pulso de entrada. De hecho, en este caso, todos los intentos de disminuir el efecto de una perturbación en la salida conllevan necesariamente a un undershoot en un momento posterior. Estos resultados son obviamente aplicables a los tratamientos de basal-bolo en DMT1, donde las comidas típicamente elevan el nivel de glucosa en la sangre antes de que la insulina actúe. En el trabajo [134 se formaliza el compromiso entre las excursiones de hiperglucemia y los eventos de hipoglucemia inherentes a las limitaciones del sistema de glucosa-insulina.

La principal contribución de [134 puede resumirse de la siguiente forma. Considere un sistema dinámico positivo perturbado mediante un pulso a $t=0$ (por ejemplo, el modelo DMT1 perturbado por una comida). Siendo $T_{1}$ y $T_{2}>T_{1}$ dos tiempos arbitrarios y definiendo $y\left(T_{1}\right)$ e $y\left(T_{2}\right)$ como las desviaciones correspondientes de la salida del sistema con respecto al punto de operación. Si se establece un límite inferior $y_{T_{2}}$ para la respuesta de salida en $T_{2}$ (por ejemplo, un nivel mínimo aceptable de glucosa posprandial), cumpliéndose que $y\left(T_{2}\right)>y_{T_{2}}$, entonces la respuesta de salida en el momento $T_{1}$ también estará limitada por un valor mínimo $y\left(T_{1}\right)>y_{T_{1}}$ (por ejemplo, el pico posprandial será al menos $y_{T_{1}}$ ). El valor de $y_{T_{1}}$ será función de la perturbación, la dinámica del sistema y los parámetros, y estará vinculado al límite establecido para $y\left(T_{2}\right)$. Aunque $T_{1}$ y $T_{2}$ son arbitrarios, es interesante alinearlos con los valores máximo y mínimo de la respuesta de salida. Esto permite predecir la compensación entre las excursiones positivas y negativas de la señal de salida. La entrada del sistema que cumple con ambos límites es un pulso en $t=0$ (en el momento en que se produce la perturbación) y cero en el resto del intervalo $\left[0, T_{2}\right]$. Esto es suponiendo que no se puede actuar con anticipación a la perturbación.

Estos resultados tienen implicaciones directas cuando se aplican a los modelos de DMT1, La Figura 3.2 ilustra la aplicación de las formulaciones expresadas en este trabajo a una combinación de basal-bolo para rechazar una perturbación producida por la 

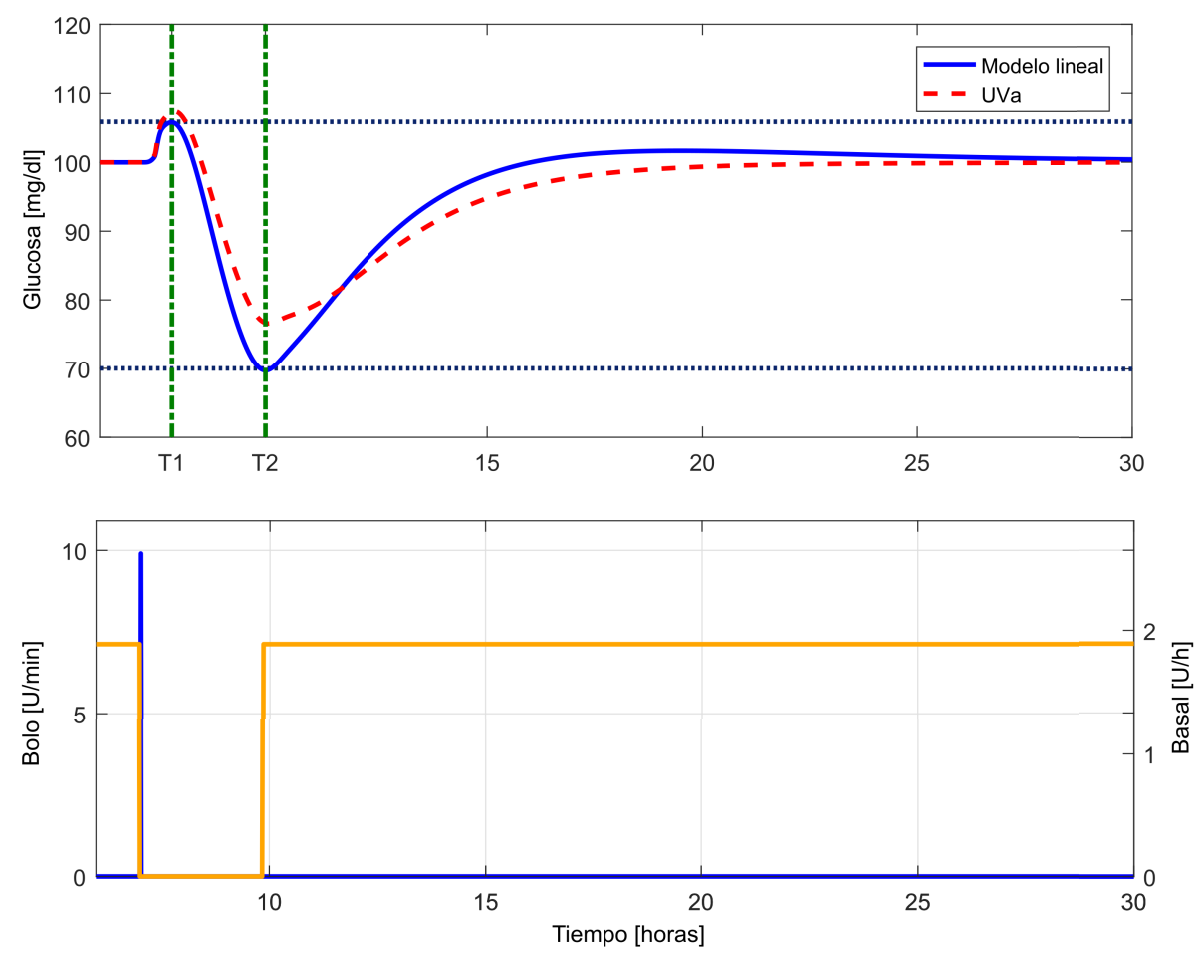

Figura 3.2: Curva ilustrativa del resultado teórico de 134. Panel superior: perfiles de glucosa (línea sólida azul corresponde al modelo UVA/Padova linealizado; línea punteada roja corresponde al sujeto virtual); las líneas horizontales punteadas indican los límites inferiores $y_{T_{1}}$ y $y_{T_{2}}$ para $T_{1}$ y $T_{2}$, respectivamente. Panel inferior: entrada de insulina (tanto basal como bolo) que cumple con ambos límites.

ingesta. Esta respuesta se obtiene para el modelo linealizado del adulto \#6 del simulador UVA/Padova. El sujeto comienza con una glucosa en estado estacionario igual a $100 \mathrm{mg} / \mathrm{dl}$, donde el suministro de insulina basal corresponde a $1.9 \mathrm{U} / \mathrm{h}$. Una comida de $30 \mathrm{~g}$ de $\mathrm{CHO}$ se ingiere en $t=0$. Según la relación CR de este paciente, el bolo de comida es igual $3 \mathrm{U}$. Asumamos por el momento que los tiempos $T_{1}=50 \mathrm{~min}$ y $T_{2}=170 \mathrm{~min}$ en los que se producen los niveles máximos y mínimos de glucosa se conocen de forma a priori. A su vez, se establece que el nivel mínimo aceptable de la excursión de glucosa en $T_{2}$ es $\underline{y_{T_{2}}}=70 \mathrm{mg} / \mathrm{dl}$. Luego, después de algunos cálculos ${ }^{2}$, se puede determinar que el nivel máximo de glucosa en $T_{1}$ será de al menos 105,9 mg/dl. Además, la entrada de control calculada que proporciona la excursión de hiperglucemia más baja en $T_{1}=50 \mathrm{~min}$ dado un valor mínimo de glucosa establecido $\underline{y_{T_{2}}}=70 \mathrm{mg} / \mathrm{dl}$ en $T_{2}=170 \mathrm{~min}$ se representa en la parte inferior de la figura. El perfil de entrada de basal-bolo consiste en un bolo de insulina a la hora de la comida y una suspensión de la basal hasta que se produzca el nivel más bajo de glucosa a $T_{2}=170 \mathrm{~min}$. Este perfil de suministro de insulina se corresponde a a la forma de un súper bolo. El bolo de insulina calculado de $9.9 \mathrm{U}$ es mucho más alto que el bolo estándar de 3U. La cantidad extra de insulina administrada a la hora de la comida compensa la cantidad de insulina basal no administrada hasta $T_{2}$. Para más detalles, tanto en el procedimiento de cálculo y en la formulación teórica, se remite al lector a [134.

Los resultados anteriores resultan muy interesantes desde el punto de vista teórico y proporcionan una forma analítica de determinar el mejor perfil de acción control. Sin

\footnotetext{
${ }^{2}$ El teorema que propone Goodwin et al. para el cálculo de la acción de control que cumple con la restricción impuesta dependerá de la respuesta impulsional del sistema a la insulina (acción de control) y a la comida (perturbación). A su vez, depende de los instantes de tiempo seleccionados y de los límites. Por estos motivos, utilizar este teorema para el cálculo de un super-bolo para la terapia a lazo abierto resulta inadecuado y no se desarrolla el cálculo realizado para el ejemplo.
} 
embargo, la suposición de que los instantes de tiempo $T_{1}$ y $T_{2}$ se conocen a priori no es realista. Por lo tanto, a pesar de garantizar las especificaciones de rendimiento, el método propuesto en 134 no puede utilizarse para determinar la mejor combinación basal-bolo en el control cotidiano de la glucosa.

La propuesta del tratamiento automático de SB basado en la IOB surge de la búsqueda de una combinación basal-bolo que sea capaz de lograr un mejor manejo de la glucosa, en particular frente a comidas con AIG. Se utiliza el concepto IOB a fin de calcular la cantidad extra de insulina para potenciar el bolo y el momento en que se debe restablecer la administración de insulina basal.

\subsubsection{Super-Bolo automático basado en IOB}

Para resolver el problema de establecer el tiempo de corte, es decir, el tiempo durante el cual se suspende la administración de insulina basal al aplicar un SB, se propone un algoritmo para el cálculo automático de un SB basado en IOB.

La idea subyacente de la propuesta es fácil e intuitiva. Se impone una restricción suave $3^{3}$ la dinámica de IOB que permite limitar la insulina administrada al sujeto. Esto consiste en fijar un valor umbral en el nivel de IOB, denominado como $\overline{I O B}$. Cuando se administra un $\mathrm{SB}$, el valor límite de IOB será excedido. Se propone que el suministro basal no se reanude hasta que el nivel de IOB alcance nuevamente el valor umbral establecido por la restricción. Como resultado, la insulina no continuará acumulándose mientras el IOB esté por encima del umbral, evitando o reduciendo así los posibles casos de hipoglucemia tardía.

Para ilustrar y comprender el funcionamiento del SB automático, se considera una comparación in-silico entre los perfiles IOB generados por un SB manual y el método propuesto. La estimación del IOB se obtiene del modelo dinámico descrito en la sección anterior, dado por la ecuación (3.7). El procedimiento de cálculo y otras consideraciones se desarrollarán más adelante.

Supóngase que tres sujetos diferentes -digamos A, B y C- están en estado estacionario bajo una tasa de insulina basal de $1 \mathrm{U} / \mathrm{h}$. Cada uno tiene un DIA aproximado de 7,4 y 3 horas respectivamente. Una hora después del inicio de la simulación se programa un bolo de insulina de $2 \mathrm{U}$ en forma de SB. Se simuló un SB manual con un tiempo de corte fijo genérico de 2 horas $\left(T_{\text {cutoff }}=2 \mathrm{~h}\right)$, creando así una inyección de insulina de $4 \mathrm{U}(2 \mathrm{U}+$ $1 \mathrm{U} / \mathrm{h} \cdot 2 \mathrm{~h}$ ). Los perfiles de IOB y de suministro de insulina correspondientes se muestran en la Fig. 3.3 (a). Las líneas punteadas indican el valor de estado estacionario (e.e.) de IOB correspondiente (según el DIA) a cada sujeto. Se puede observar que para el sujeto A el suministro basal vuelve antes de que el IOB estimado alcance el nivel de e.e., por lo que se produce un apilamiento de insulina (línea discontinua). Este mayor transitorio en la IOB no es deseable porque podría producir niveles bajos de glucosa en el futuro, incluso hipoglucemia. En el caso del sujeto C, el nivel de IOB cae por debajo del valor de e.e. antes del restablecimiento de la insulina basal (línea punteada), dando lugar a un undershoot no deseado. El perfil de IOB del sujeto B (línea continua) no presenta una acumulación o déficit excesivo de insulina ya que el suministro de insulina se reanuda cuando el nivel de IOB está cerca del valor basal. Este tipo de perfil se consigue de forma aleatoria usando un SB manual, ya que se selecciona un $T_{\text {cutoff }}$ arbitrario.

El SB automático basado en la IOB se simuló en los mismos tres sujetos bajo las mismas condiciones. Se puede ver en la Fig. 3.3 (b) que se evitan los transitorios indeseados o los undershoot en los tres casos, ya que el suministro basal se retoma cuando el nivel de IOB es igual al IOB de estado estacionario. Aún así, se puede observar un pequeño

\footnotetext{
${ }^{3}$ Se define una restricción suave ya que al tratarse de un algoritmo a lazo abierto y al contar con incertidumbre en el modelo de estimación de IOB no es de interés que se cumpla estrictamente.
} 

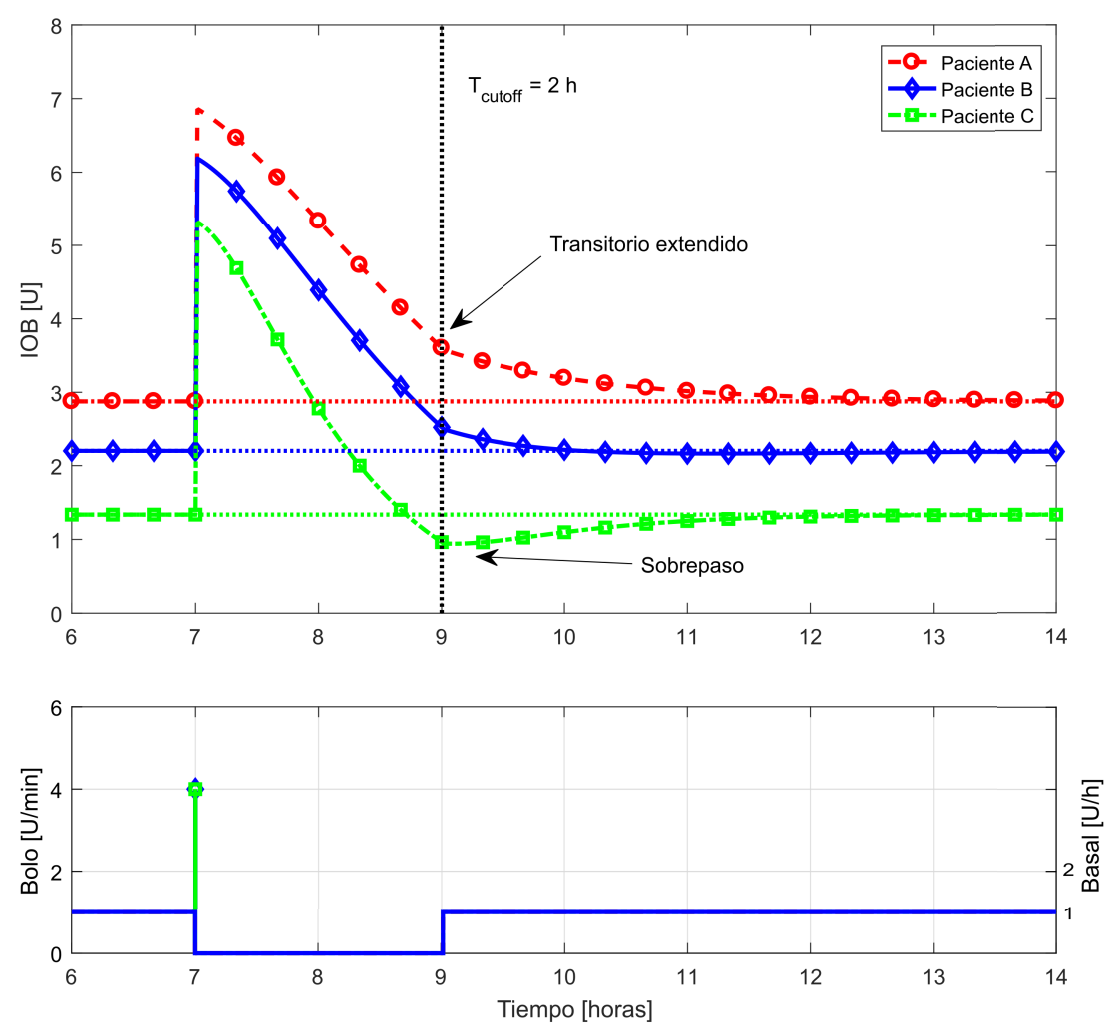

(a) SB manual con un tiempo de corte de $2 \mathrm{~h}$.
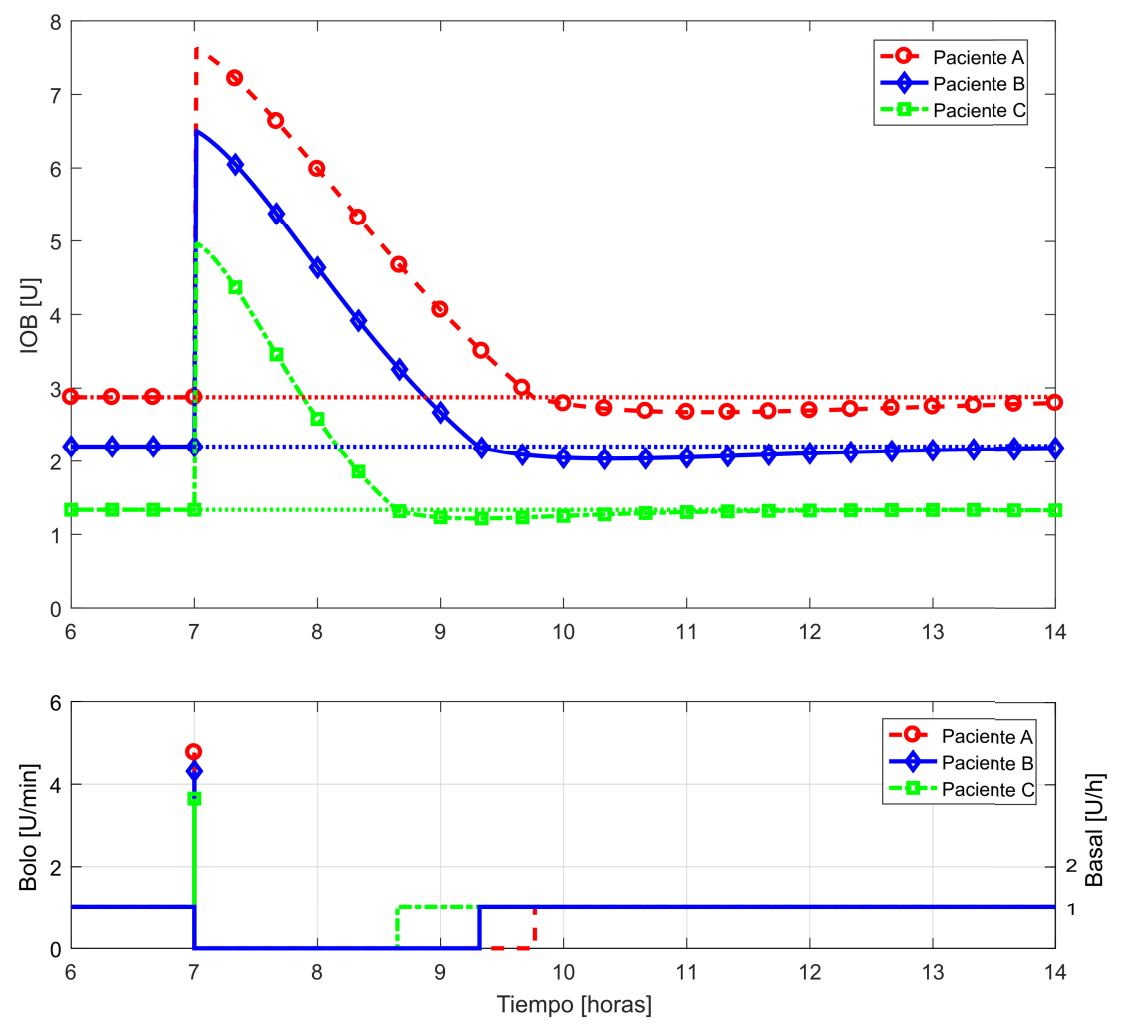

(b) SB automático basado en IOB adaptado según el DIA de cada sujeto.

Figura 3.3: Perfiles de IOB para diferentes sujetos generados a partir de un SB manual y del SB automático basado en IOB. 
transitorio debido a la dinámica de segundo orden del sistema. Ésta puede evitarse eligiendo el $\overline{I O B}$ ligeramente más alto que el valor de estado estacionario. La inyección de insulina subcutánea para cada caso puede verse en la parte inferior de la Fig. 3.3 (b), donde es evidente cómo se adapta el tiempo de corte en función del DIA para compensar la variabilidad del mismo entre diferentes sujetos.

\section{Procedimiento de cálculo}

La propuesta consiste en que la bomba ejecute un algoritmo cuando el usuario desee inyectar un determinado bolo en forma de SB. El algoritmo calcula una dosis de insulina $I[t]$ (basal y bolo) teniendo en cuenta la estimación de la IOB según la siguiente metodología:

$$
I[t]=I_{\text {basal }}[t]+I_{S B}[t]
$$

con suministro basal definido como

$$
I_{\text {basal }}[t]=\left\{\begin{array}{ll}
0 & \text { si } \quad T_{\text {bolus }} \leq t \leq T_{\text {bolus }}+T_{\text {cutoff }} \\
B R[t] \cdot T_{s} & \text { c.c. }
\end{array},\right.
$$

y al instante del suministro del super-bolo

$$
I_{S B}[t]=\left\{\begin{array}{ll}
I_{\text {bolus }}+\bar{I}_{b} & \text { si } \\
0 & \text { c.c. }
\end{array} \quad t=T_{\text {bolus }}\right.
$$

donde:

- $I[t]$ es la dosis total de insulina $(\mathrm{U})$

- $B R[t]$ es el perfil de insulina basal del sujeto (U/min)

- $T_{s}$ es el tiempo de muestreo de la bomba

- $\bar{I}_{b}[t]$ es la suma de insulina basal entre el tiempo $T_{\text {bolus }}$ y $T_{\text {bolus }}+T_{\text {cutoff }}$, dado por

$$
\bar{I}_{b}=\sum_{k=T_{\text {bolus }} / T_{s}}^{\left(T_{\text {bolus }}+T_{\text {cutoff }}\right) / T_{s}} B R[k] \cdot T_{s} .
$$

El valor del bolo para la compensación de carbohidratos original a ser administrado $I_{\text {bolus }}$ puede ser calculado como de costumbre basado en la relación insulina-carbohidratos del usuario y la estimación de CHO de se ingerirá, o por medio de cualquier otro método.

La infusión basal dependerá de una restricción $\overline{I O B}[t]$ impuesta sobre la IOB . El tiempo durante el cual se interrumpirá la infusión de insulina basal puede predecirse a partir de la respuesta dinámica del sistema IOB debido a una entrada en forma de SB dada por (3.8). Una realización discreta muestreada a $t=k T_{s}$ del modelo validado 3.5 se utiliza para la estimación del IOB:

$$
\begin{aligned}
x[k+1] & =A x[k]+B u[k] \\
I O B[k] & =C x[k]
\end{aligned}
$$

con

$$
A=\left[\begin{array}{cc}
\left(1-K_{\mathrm{DIA}} \cdot T_{s}\right) & 0 \\
K_{\mathrm{DIA}} \cdot T_{s} & \left(1-K_{\mathrm{DIA}} \cdot T_{s}\right)
\end{array}\right], B=\left[\begin{array}{c}
T s \\
0
\end{array}\right], C=\left[\begin{array}{ll}
1 & 1
\end{array}\right] .
$$


Se desea calcular el perfil de IOB después de la entrega del SB. Las trayectorias de los estados $x[k]$ en cada muestra pueden expresarse mediante la solución no homogénea del sistema lineal invariante del tiempo $(3.12)$ como

$$
x[k]=A^{k-k_{0}} x_{0}+\sum_{\tau=k_{0}}^{k-1} A^{k-1-\tau} B u[\tau],
$$

donde la entrada definida como un bolo esta dada por ec. 3.10, reescrita como

$$
u[k]= \begin{cases}I_{S B} & \text { if } \\ 0 & \text { else }\end{cases}
$$

Considerando que $T_{\text {bolus }}=t_{0}=k_{0} T_{s}$, las trayectorias de $x[t]$ desde $t_{0}$ a $t_{0}+T_{\text {cutoff }}$ pueden ser calculadas a partir de la respuesta homogénea debido a la interrupción de insulina basal y a partir de la respuesta forzada a la entrada (3.15):

$$
x\left[t_{0}+T_{\text {cutoff }}\right]=A^{T_{\text {cutoff }} / T_{s}} x_{0}+A^{T_{\text {cutoff } f} / T_{s}-1} B \cdot I_{S B},
$$

donde $x_{0}$ corresponde al valor de los estados en el tiempo $T_{b o l u s}$. De esta forma, la estimación de la IOB resulta en:

$$
I O B\left[t_{0}+T_{\text {cutoff }}\right]=C A^{T_{\text {cutoff }} / T_{s}} x_{0}+C A^{T_{\text {cutoff } / T_{s}}-1} B \cdot I_{S B} .
$$

Aunque el método permite seleccionar cualquier $\overline{I O B}[t]$, se propone aquí utilizar el valor IOB de estado estacionario o basal $I O B_{\text {basal }}$ para evitar undershoots o retardos innecesarios en la señal IOB como se ilustró anteriormente. Por lo tanto, cuando se reanude el suministro basal, la insulina que quede en el cuerpo será igual al equilibrio de (3.12) dado por el perfil de insulina basal correspondiente al tiempo $T_{\text {bolus }}+T_{\text {cutoff }}{ }^{4}$, esto es

$$
I O B_{\text {basal }}=2 \cdot B R\left[T_{\text {bolus }}+T_{\text {cutoff }}\right] / K_{\text {DIA }} .
$$

Finalmente, el cálculo de $T_{\text {cutoff }}$ y la cantidad extra de bolo $\bar{I}_{b}$ a aplicar resulta de la resolución de la ecuación:

$$
I O B_{\text {basal }}=C A^{T_{\text {cutoff }}} x_{0}+C A^{T_{\text {cutoff }}-T_{s}} B\left(I_{\text {bolus }}+\sum_{t=0}^{T_{\text {cutoff }}} B R[t] \cdot T_{s}\right) .
$$

Esta ecuación puede ser resuelta numéricamente, por ejemplo, a través del método NewtonRaphson, el cual converge rápidamente y tiene un bajo costo computacional, entre otros.

El pseudocódigo resultante puede verse en los algoritmos 1 y 2 2. Los parámetros Tolerance (mínimo error relativo) y Max_iter (cantidad máxima de iteraciones) determinan la convergencia del algoritmo y pueden ajustarse como se desee, mientras que el valor inicial en $t_{0}$ debe ser del orden del valor $D I A$. El estado "Advertencia" implica que hubo un error en la convergencia, en consecuencia se puede establecer un tiempo de corte genérico, utilizar el tiempo de corte calculado hasta la interrupción o evitar la creación del SB, dependiendo del criterio elegido.

Vale la pena enfatizar que este cálculo puede ser fácilmente realizado por software en cualquier bomba moderna disponible en el mercado. La precisión del método depende tanto de la discretización de la bomba -ya que heredará las características físicas de cada bomba- como de la estimación del DIA. La velocidad de cálculo depende del hardware de la bomba y es independiente de la actualización del DIA en caso de que este sea

\footnotetext{
${ }^{4}$ se considera perfiles de insulina basal variantes en el tiempo, por lo que la insulina basal en el tiempo en que se suministra el bolo $T_{\text {bolus }}$ y se produce el corte puede diferir de cuando se reanuda en el tiempo $T_{\text {bolus }}+T_{\text {cutoff }}$, por lo tanto se considera el e.e. debido a $B R\left[T_{\text {bolus }}+T_{\text {cutoff }}\right]$
} 
estimado y basado en medición. De esta manera, el [SB podría añadirse como una opción extra de administración de bolo sólo dependiendo del DIA del usuario y de su perfil basal. Además, esta técnica puede combinarse con la sustracción del exceso de IOB del bolo o con cualquier otro método que implique restricciones de IOB (por ejemplo, [93]). El algoritmo puede implementarse con algunas consideraciones de seguridad, como pedir confirmación por parte del paciente de la cantidad de insulina y el tiempo de corte, limitando el tiempo de corte al máximo deseado, entre otros aspectos que parezcan necesarios.
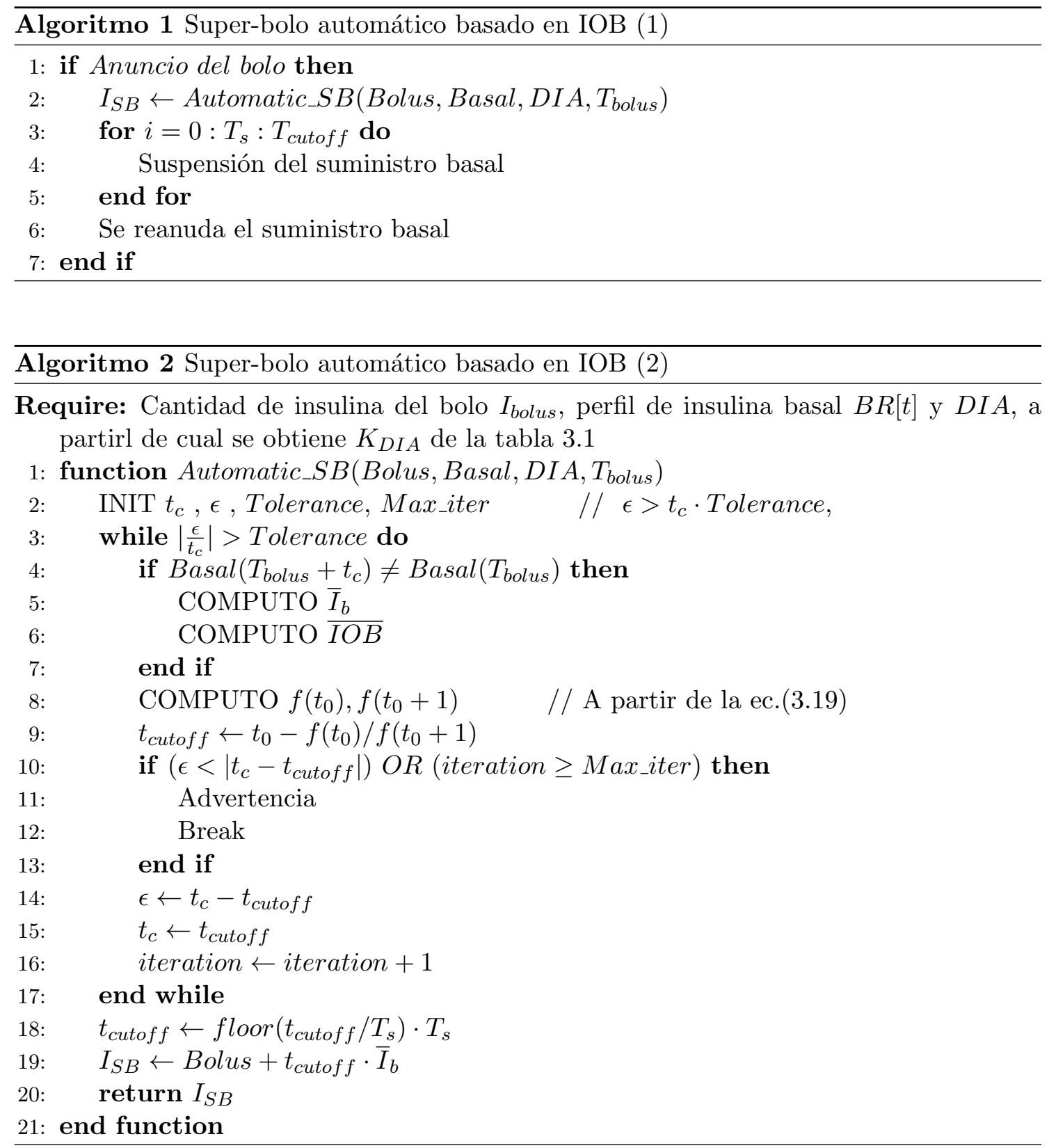

\subsubsection{Resultados}

Para evaluar el rendimiento del tratamiento a lazo abierto propuesto, se realizaron una serie de pruebas en la plataforma de simulación UNLP. Para evaluar el rendimiento del algoritmo se consideró una comparación con el tratamiento estándar de basal-bolo óptimo y la implementación de un super-bolo manual. Se consideró la cohorte de sujetos virtuales del UVA/Padova de 10 adultos, 10 adolescentes y 10 niños sobre dos clases de escenarios. 


\section{Definición de los escenarios}

En un primera instancia, se definieron escenarios de una comida única para la evaluación del periodo posprandial. Se considera una comida de AIG de carbohidratos puros. La absorción de glucosa evoluciona según el modelo de Dalla Man descrito en [135]. Se suministra la comida al sujeto en el comienzo de una simulación de 10 horas. Los sujetos comienzan en un equilibrio inicial igual a su valor de glucosa en ayunas y la tasa de insulina basal que lo mantiene en ese nivel de glucosa. Se seleccionaron 4 tamaños de comidas diferentes: $25 \mathrm{~g}, 50 \mathrm{~g}, 75 \mathrm{~g}$ y $100 \mathrm{~g}$. Para cada escenario de comida única se considera, además del tratamiento estándar y el SB automático, un SB manual. Dado que no existe una forma estándar de definirlo, se consideró un tiempo de corte fijo para cada tamaño de comida. Para una comparación justa, estos tiempos de corte fijos se definieron a partir de las media de los tiempos $T_{\text {cutoff }}$ obtenido por el tratamiento del SB automático. Los tiempos de corte medios resultantes para las comidas de 25, 50, 70 y 100 gramos para toda la cohorte fueron 135, 166, 186 y 200 minutos respectivamente.

A fin de evaluar la robustez de la metodología propuesta, se consideraron tres escenarios de un solo día (30 horas 5 ) que introducen variabilidad intra-paciente. En estos escenarios realistas se consideró la variabilidad diurna de los parámetros del sistema que describen la sensibilidad a la insulina y la absorción de la glucosa. Aquí se simularon los pacientes bajo perfiles basales no ideales y variables y factores CR de acuerdo a como se presento en el Capítulo 2 Sección 2.2.2. A su vez, se tiene en cuenta el efecto de las diferentes tasas de aparición de la glucosa con el objetivo de comparar el rendimiento del SB automático cuando se enfrentan tanto a comidas de AIG como de BIG. Se definieron 3 escenarios de un solo día con comidas en el desayuno a las $7 \mathrm{~h}$, almuerzo a las $13 \mathrm{~h}$, merienda a las $17 \mathrm{~h}$ y cena a las $21 \mathrm{~h}$ para toda la cohorte de sujetos:

- Escenario 1: cuatro comidas de AIG de carbohidratos puro que contienen 80g, 40g, 10g y $70 \mathrm{~g}$ respectivamente (absorción de glucosa según [135]).

- Escenario 2: cuatro comidas mixtas de AIG con una suma total de 200g. Las tasas de aparición de glucosa de cada comida pueden observarse en la Fig. 3.4 (a).

- Escenario 3: cuatro comidas mixtas de BIG con una suma total de 200g. Las tasas de aparición de glucosa de cada comida pueden observarse en la Fig. 3.4 (b).

Las métricas de evaluación para los escenarios de una comida única se presentan en la tabla 3.2 y para los escenarios de un solo día en la tabla 3.3. Estas métricas se encuentran basadas en el informe consensuado presentado en [136]. Para la comparación entre los tratamientos, la prueba de hipótesis se realizó utilizando una prueba de Wilcoxon de rango con signo de 2 muestras, considerando $\rho<0,05$ como estadísticamente significativo. El análisis cualitativo de la calidad del control se puede visualizar en el gráfico CVGA [86]. Las categorías del CVGA representan niveles de control glucémico de la siguiente forma:

- A-zone: Preciso.

- Lower/Upper B-zones: Desviación benigna a la hipo/hiperglucemia.

- B-zone: Control benigno.

- Upper/Lower C-zone: Sobrecorreción de hipo/hiperglucemia.

- Lower/Upper D-zone: Falla para controlar hipo/hiperglucemia.

- E-zone: Control erróneo.

\footnotetext{
${ }^{5}$ Se consideran 6 horas más después de las 24 horas para tener en cuenta el periodo posprandial de la cena
} 


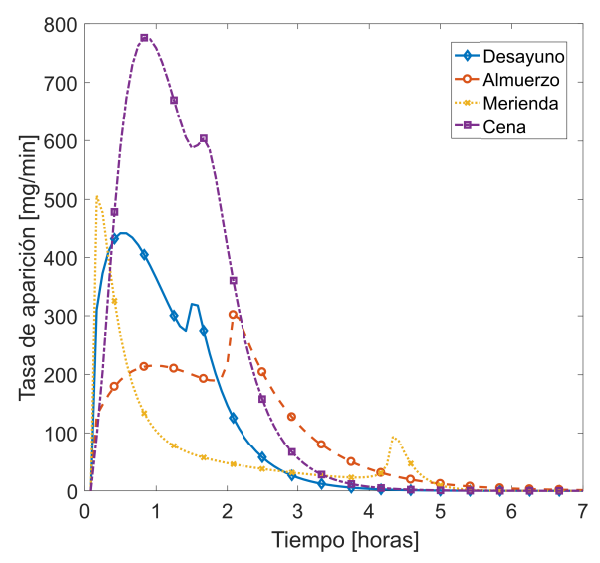

(a) Comidas mixtas de AIG del Escenario

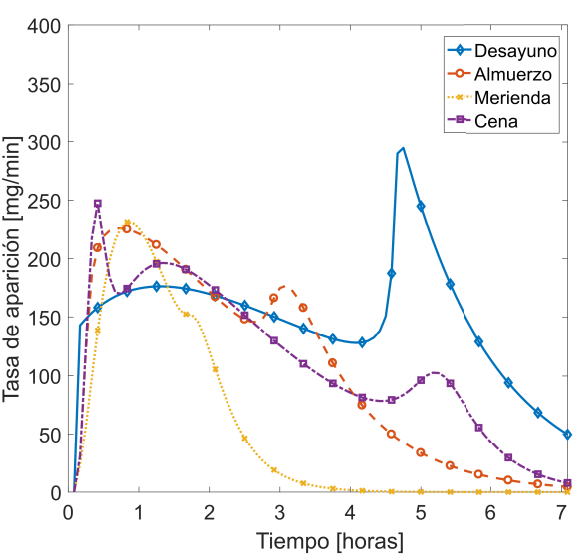

(b) Comidas mixtas de BIG del Escenario

Figura 3.4: Tasa de aparición de glucosa de las comidas mixtas utilizadas en los escenarios 2 y 3 .

Tabla 3.2: Comparación entre el tratamiento estándar y el SB automático para los escenarios de una comida única.

\begin{tabular}{|c|c|c|c|c|c|c|c|c|}
\hline & $\begin{array}{l}\text { GP Media } \\
(\mathrm{mg} / \mathrm{dl})\end{array}$ & $\begin{array}{c}\mathrm{GP}<50 \mathrm{mg} / \mathrm{dl} \\
(\% \text { tiempo })\end{array}$ & $\begin{array}{c}\mathrm{GP}<60 \mathrm{mg} / \mathrm{dl} \\
(\% \text { tiempo })\end{array}$ & $\begin{array}{c}\mathrm{GP}<70 \mathrm{mg} / \mathrm{dl} \\
(\% \text { tiempo })\end{array}$ & $\begin{array}{c}\mathrm{GP} \in[70,180] \mathrm{mg} / \mathrm{dl} \\
(\% \text { tiempo })\end{array}$ & $\begin{array}{c}\mathrm{GP}>180 \mathrm{mg} / \mathrm{dl} \\
(\% \text { tiempo })\end{array}$ & $\begin{array}{c}\mathrm{GP}>250 \mathrm{mg} / \mathrm{dl} \\
\quad(\% \text { tiempo })\end{array}$ & $\begin{array}{c}\mathrm{GP}>300 \mathrm{mg} / \mathrm{dl} \\
(\% \text { tiempo })\end{array}$ \\
\hline \multicolumn{9}{|c|}{ Comida única: $25 \mathrm{~g}$} \\
\hline Estándar & $135,43 \pm 12,48$ & $0 \pm 0$ & $0 \pm 0$ & $0,6 \pm 3,27$ & $96,03 \pm 6,82$ & $3,37 \pm 6,33$ & $0,27 \pm 1,49$ & $0 \pm 0$ \\
\hline SB automático & $136,46 \pm 6,83$ & $0 \pm 0$ & $0 \pm 0$ & $0,32 \pm 1,75$ & $97,72 \pm 5,21$ & $1,96 \pm 5,03$ & $0,15 \pm 0,84$ & $0 \pm 0$ \\
\hline$\rho$ - valor & 0,517 & - & - & 0,5 & $<0,001^{\star}$ & $0,001^{\star}$ & 0,5 & - \\
\hline \multicolumn{9}{|c|}{ Comida única: $50 \mathrm{~g}$} \\
\hline Estándar & $132,66 \pm 24,25$ & $0 \pm 0$ & $1,16 \pm 6,33$ & $2,13 \pm 10,46$ & $89,67 \pm 12,27$ & $8,2 \pm 8,61$ & $1,23 \pm 4,33$ & $0,39 \pm 2,15$ \\
\hline SB automático & $134,92 \pm 15,5$ & $0 \pm 0$ & $0 \pm 0$ & $0,42 \pm 1,65$ & $93,53 \pm 8,32$ & $6,05 \pm 8,31$ & $0,86 \pm 3,64$ & $0,3 \pm 1,62$ \\
\hline$\rho$ - valor & 0,066 & - & 0,5 & 0,5 & $0,001^{\star}$ & $0,001^{\star}$ & 0,063 & 0,5 \\
\hline \multicolumn{9}{|c|}{ Comida única: $75 \mathrm{~g}$} \\
\hline Estándar & $131,15 \pm 35,88$ & $1,15 \pm 6,28$ & $2,51 \pm 9,75$ & $5,92 \pm 14,48$ & $81,9 \pm 15,59$ & $12,18 \pm 9,94$ & $3,03 \pm 5,73$ & $1,25 \pm 4,31$ \\
\hline SB automático & $134,22 \pm 25,61$ & $0 \pm 0$ & $0,28 \pm 1,54$ & $0,97 \pm 4,15$ & $89,51 \pm 11,95$ & $9,51 \pm 9,94$ & $2,4 \pm 5,05$ & $0,95 \pm 3,8$ \\
\hline$\rho$ - valor & $0,017^{\star}$ & 0,5 & 0,125 & $0,008^{\star}$ & $<0,001^{\star}$ & $<0,001^{\star}$ & $0,002^{\star}$ & 0,063 \\
\hline \multicolumn{9}{|c|}{ Comida única: $100 \mathrm{~g}$} \\
\hline Estándar & $131,45 \pm 47,26$ & $3,37 \pm 10,15$ & $6,2 \pm 14,31$ & $9,5 \pm 17,02$ & $75,12 \pm 18,23$ & $15,38 \pm 11,26$ & $4,77 \pm 7,15$ & $2,62 \pm 5,31$ \\
\hline SB automático & $135,34 \pm 35,93$ & $0,76 \pm 4,15$ & $1,14 \pm 5,5$ & $1,85 \pm 7,05$ & $85,46 \pm 14,96$ & $12,68 \pm 11,76$ & $3,77 \pm 6,26$ & $2,09 \pm 4,88$ \\
\hline$\rho$ - valor & $0,011^{\star}$ & $0,031^{\star}$ & $0,008^{\star}$ & $0,002^{\star}$ & $<0,001^{\star}$ & $<0,001^{\star}$ & $0,003^{\star}$ & $0,002^{\star}$ \\
\hline
\end{tabular}

Tabla 3.3: Comparación entre el tratamiento estándar y el SB automático para los escenarios de un día.

\begin{tabular}{|c|c|c|c|c|c|c|c|c|}
\hline & $\begin{array}{l}\text { GP Media } \\
(\mathrm{mg} / \mathrm{dl})\end{array}$ & $\begin{array}{c}\mathrm{GP}<50 \mathrm{mg} / \mathrm{dl} \\
(\% \text { tiempo })\end{array}$ & $\begin{array}{c}\mathrm{GP}<60 \mathrm{mg} / \mathrm{dl} \\
(\% \text { tiempo })\end{array}$ & $\begin{array}{c}\mathrm{GP}<70 \mathrm{mg} / \mathrm{dl} \\
(\% \text { tiempo })\end{array}$ & $\begin{array}{c}\mathrm{GP} \in[70,180] \mathrm{mg} / \mathrm{dl} \\
(\% \text { tiempo })\end{array}$ & $\begin{array}{c}\mathrm{GP}>180 \mathrm{mg} / \mathrm{dl} \\
(\% \text { tiempo })\end{array}$ & $\begin{array}{c}\mathrm{GP}>250 \mathrm{mg} / \mathrm{dl} \\
(\% \text { tiempo })\end{array}$ & $\begin{array}{c}\mathrm{GP}>300 \mathrm{mg} / \mathrm{dl} \\
\quad(\% \text { tiempo })\end{array}$ \\
\hline \multicolumn{9}{|l|}{ Escenario 1} \\
\hline Estándar & $127,45 \pm 30,49$ & $3,79 \pm 7,52$ & $6,37 \pm 11,65$ & $9,57 \pm 14,48$ & $79,46 \pm 17,46$ & $10,97 \pm 10,91$ & $2,45 \pm 4,85$ & $1,04 \pm 3,15$ \\
\hline SB automático & $133 \pm 23,56$ & $0,44 \pm 1,43$ & $1,9 \pm 4,05$ & $4,21 \pm 6,18$ & $86,29 \pm 11,73$ & $9,5 \pm 10,3$ & $2,15 \pm 5,03$ & $0,84 \pm 3,7$ \\
\hline$\rho$ - value & 0,229 & $0,007^{\star}$ & $0,008^{\star}$ & $0,028^{\star}$ & $0,007^{\star}$ & 0,3 & 0,57 & 0,563 \\
\hline \multicolumn{9}{|l|}{ Escenario 2} \\
\hline Estándar & $117,56 \pm 36,94$ & $1,2 \pm 2,4$ & $3,82 \pm 5,82$ & $9,21 \pm 6,6$ & $80,52 \pm 7,94$ & $10,27 \pm 3,95$ & $0,17 \pm 0,54$ & $0 \pm 0$ \\
\hline SB automático & $117,25 \pm 31,5$ & $0,67 \pm 1,45$ & $2,74 \pm 4,63$ & $5,99 \pm 6,48$ & $86,23 \pm 9,2$ & $7,78 \pm 4,92$ & $0,07 \pm 0,21$ & $0 \pm 0$ \\
\hline$\rho$ - value & 0,432 & 0,125 & 0,063 & $0,004^{\star}$ & $0,001^{\star}$ & $0,001^{\star}$ & 0,5 & - \\
\hline \multicolumn{9}{|l|}{ Escenario 3} \\
\hline Estándar & $117,96 \pm 12,05$ & $0 \pm 0$ & $0 \pm 0$ & $0,45 \pm 1,42$ & $99,55 \pm 1,42$ & $0 \pm 0$ & $0 \pm 0$ & $0 \pm 0$ \\
\hline SB automático & $120,35 \pm 10,76$ & $0 \pm 0$ & $0 \pm 0$ & $0,47 \pm 1,47$ & $99,04 \pm 1,59$ & $0,49 \pm 0,93$ & $0 \pm 0$ & $0 \pm 0$ \\
\hline$\rho$ - value & $0,014^{\star}$ & - & - & - & - & - & - & - \\
\hline
\end{tabular}

En las Figuras 3.5 3.8 se muestran los resultados para los escenarios de una comida única. Los distintos tratamientos se distinguen por el color, y el grupo etario de la población por la forma, siendo cuadrados para los adultos, rombos para los adolescentes y círculos para los niños. La comparación entre el perfil de glucosa media más una desviación estándar (DE) obtenida por los tratamientos para adultos, adolescentes y niños se muestra para 


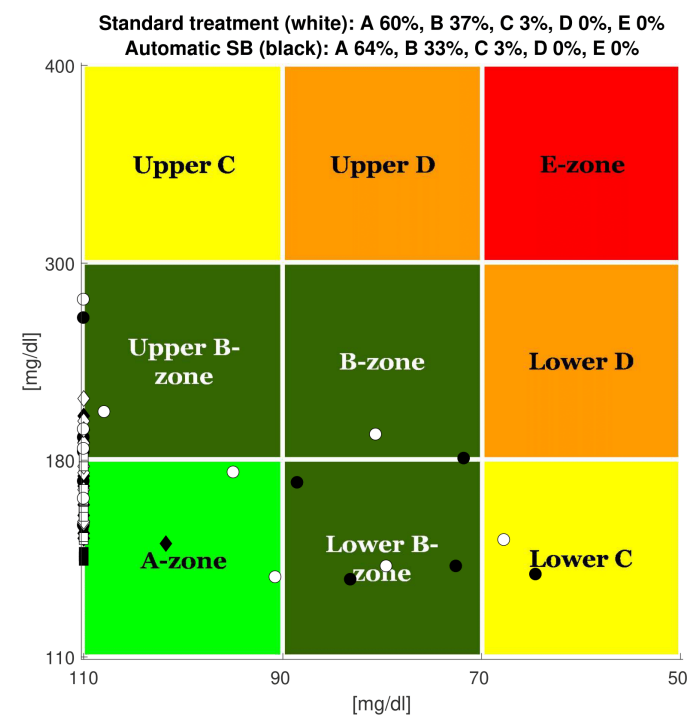

(a) Tratamiento estándar (marcadores blancos) vs. SB automático (marcadores negros).

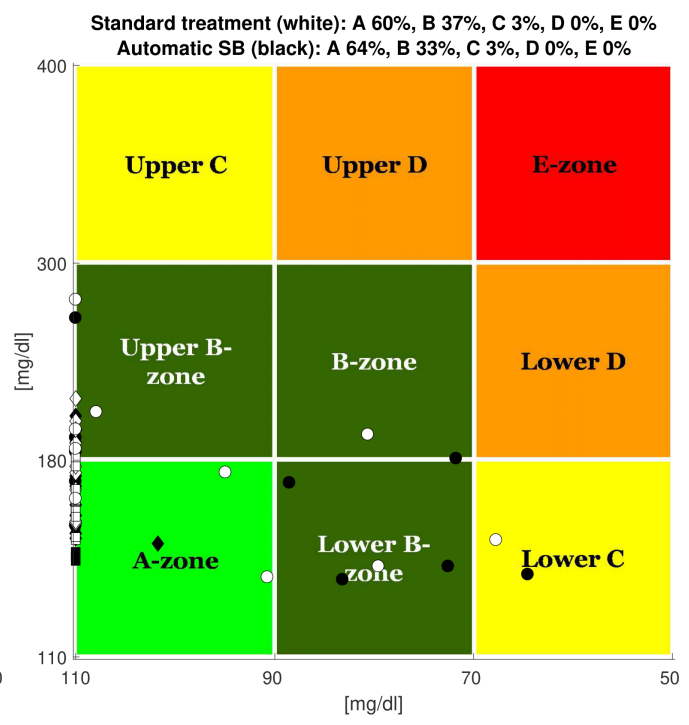

(b) SB manual (marcadores blancos) vs. SB automático (marcadores negros).

Figura 3.5: CVGA para el escenario de comida única de $25 \mathrm{~g}$. Comparación entre los tratamientos en adultos (marcadores cuadrados), adolescentes (marcadores romboidales) y niños (marcadores circulares).

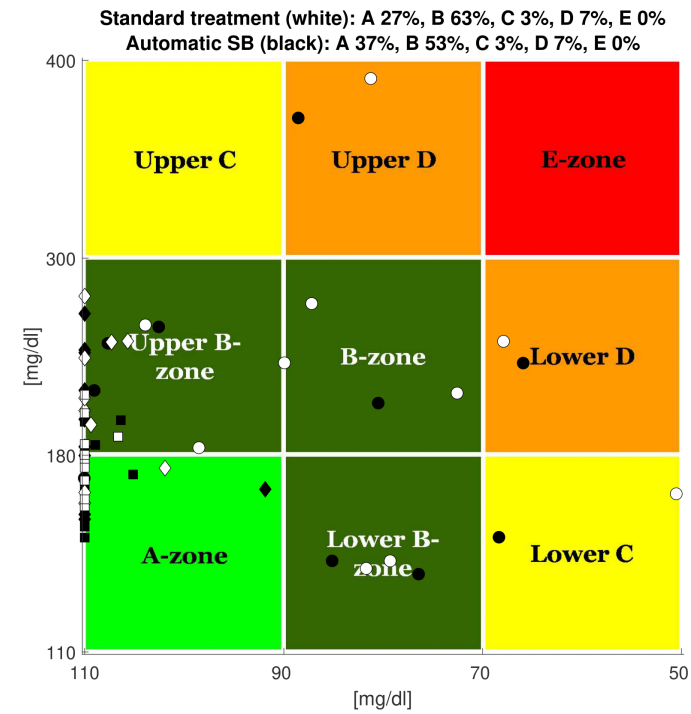

(a) Tratamiento estándar (marcadores blancos) vs. SB automático (marcadores negros).

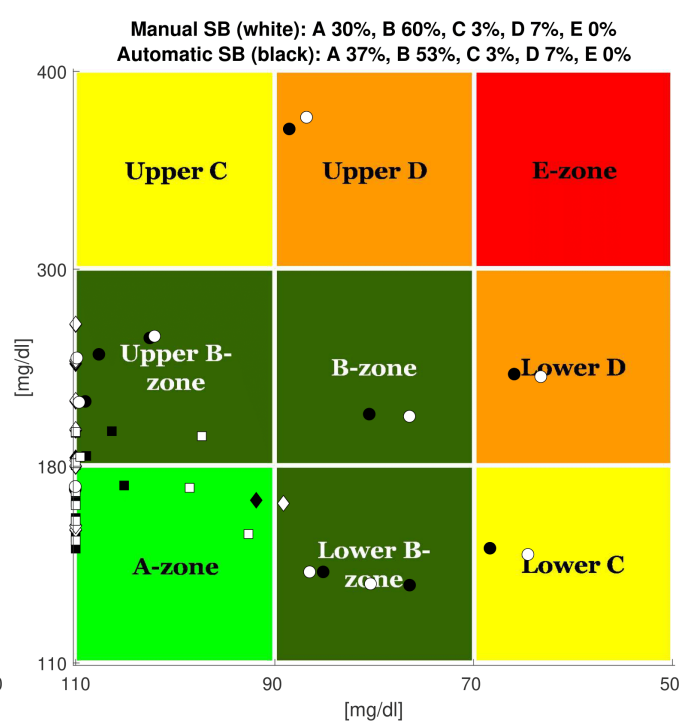

(b) SB manual (marcadores blancos) vs. SB automático (marcadores negros).

Figura 3.6: CVGA para el escenario de comida única de 50g. Comparación entre los tratamientos en adultos (marcadores cuadrados), adolescentes (marcadores romboidales) y niños (marcadores circulares).

diferentes contenidos de carbohidratos en las Fig. 3.9, 3.10 y 3.11 respectivamente.

Las gráficas CVGA correspondientes para los escenarios de un solo día se muestran en la Fig. 3.12. Los perfiles de glucosa media de los escenarios de un solo día se muestran en la Fig. 3.13 . El área sombreada indica el rango euglucémico de glucosa en sangre (GP $\in[70-$ $180] \mathrm{mg} / \mathrm{dl})$. 


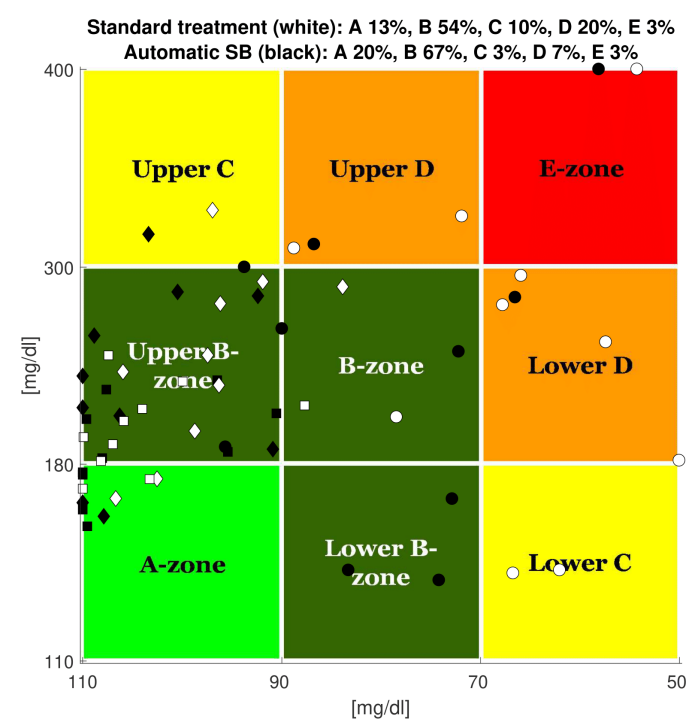

(a) Tratamiento estándar (marcadores blancos) vs. SB automático (marcadores negros).

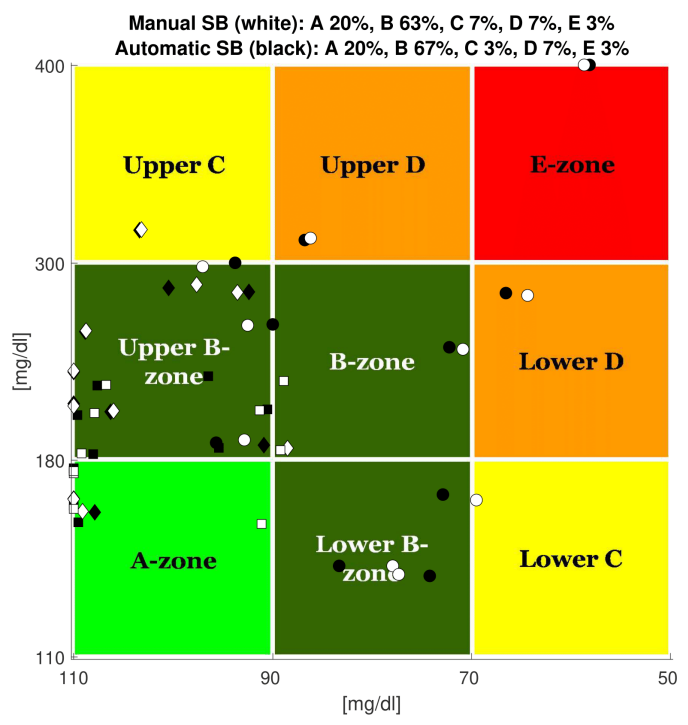

(b) SB manual (marcadores blancos) vs. SB automático (marcadores negros).

Figura 3.7: CVGA para el escenario de comida única de $75 \mathrm{~g}$. Comparación entre los tratamientos en adultos (marcadores cuadrados), adolescentes (marcadores romboidales) y niños (marcadores circulares).

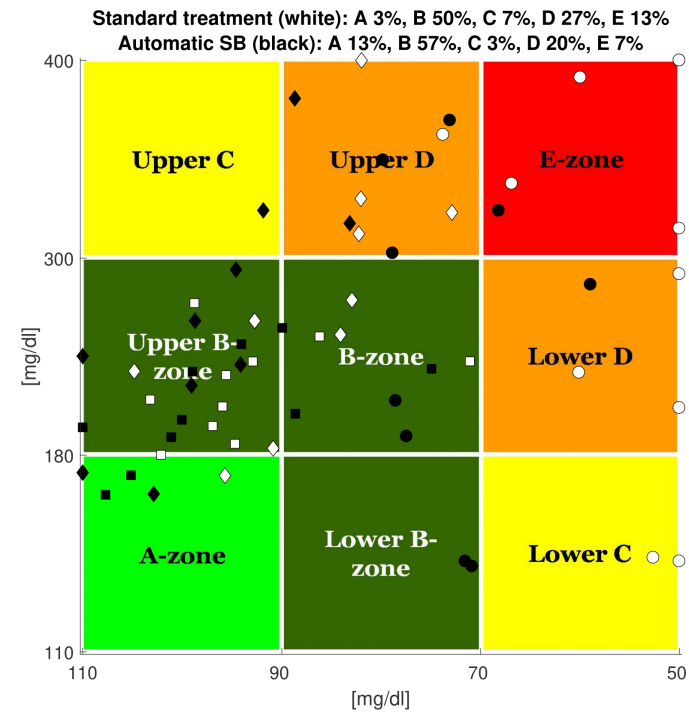

(a) Tratamiento estándar (marcadores blancos) vs. SB automático (marcadores negros).

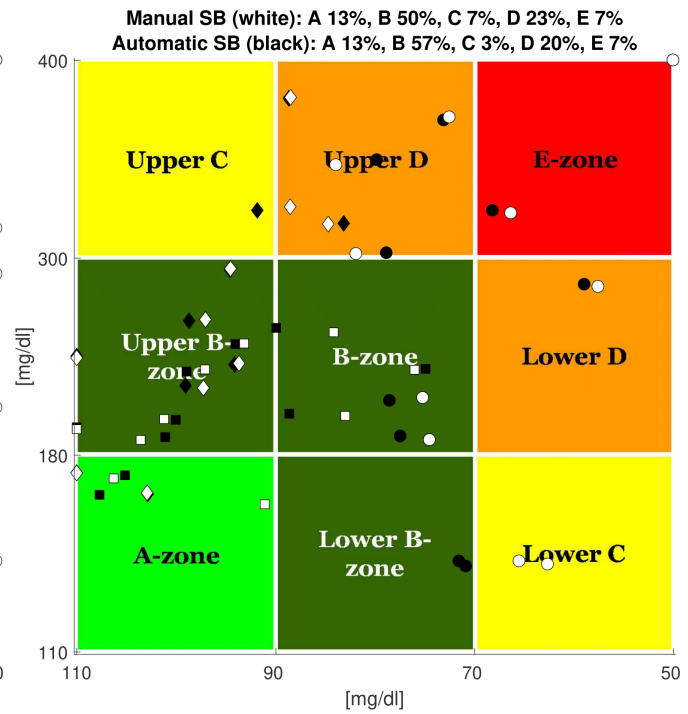

(b) SB manual (marcadores blancos) vs. SB automático (marcadores negros).

Figura 3.8: CVGA para el escenario de comida única de 100g. Comparación entre los tratamientos en adultos (marcadores cuadrados), adolescentes (marcadores romboidales) y niños (marcadores circulares).

\section{Análisis de los resultados}

A partir de los resultados obtenidos en los escenarios de una comida única, se puede observar que el método propuesto mejoró el porcentaje de tiempo en la euglucemia (tratamiento estándar vs. SB automático) de forma significativa para todas las comidas (comida de 25g: 96,03 $\pm 6,82$ vs. $97,72 \pm 5,21, \rho \simeq 0$; comida de 50g: 89,67 $\pm 12,27$ vs. $93,53 \pm 8,32, \rho \simeq 0$; comida de $75 \mathrm{~g}: 81,9 \pm 15,59$ vs. $89,51 \pm 11,95, \rho \simeq 0$; comida de $100 \mathrm{~g}:$ $75,12 \pm 18,23$ vs. $85,46 \pm 14,96, \rho<0,001)$, redujo considerablemente el porcentaje de 

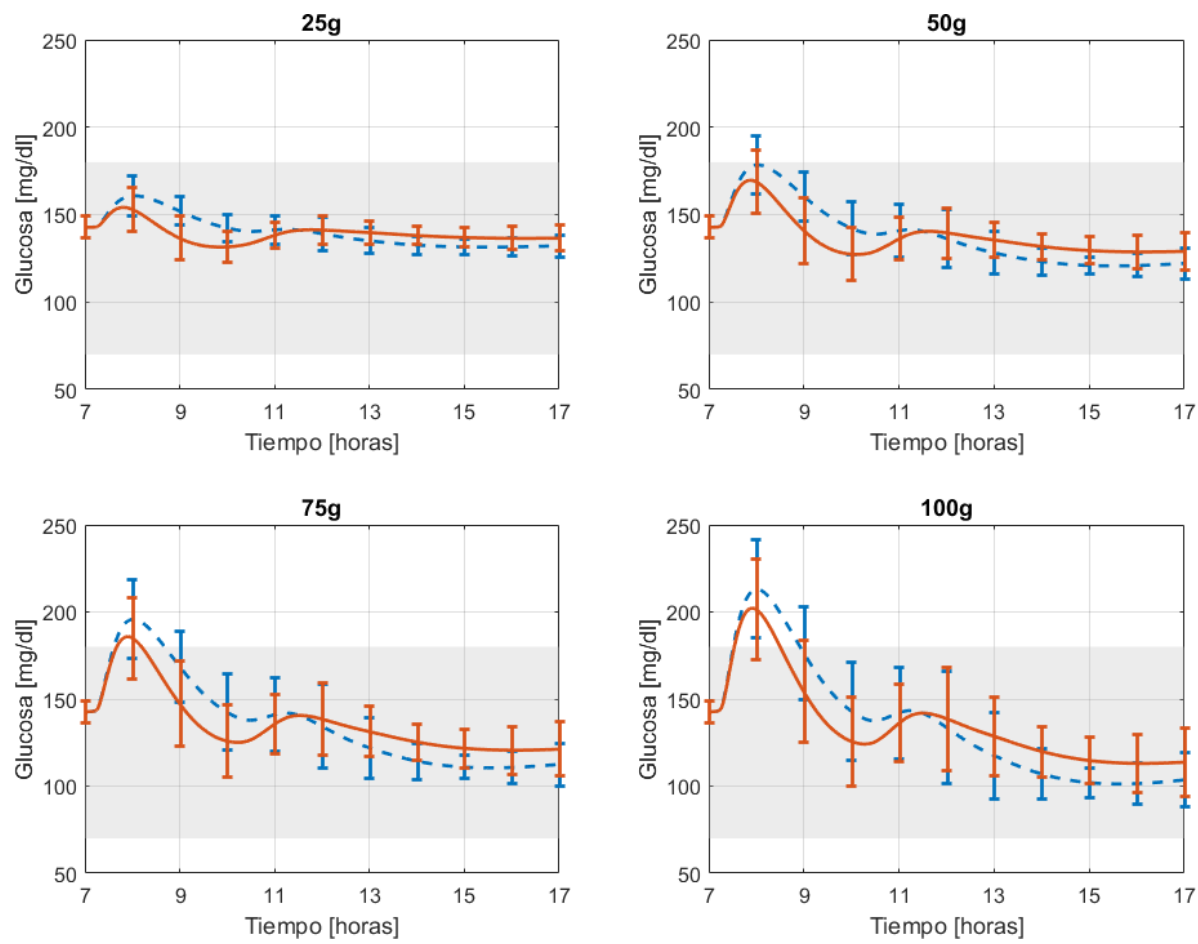

Figura 3.9: Respuesta de glucosa media \pm DE para los 10 adultos en el escenario de comida única. La línea azul a rayas corresponde al tratamiento estándar, la línea naranja sólida al SB automático. El área gris indica el rango euglucémico [70-180]mg/dl.
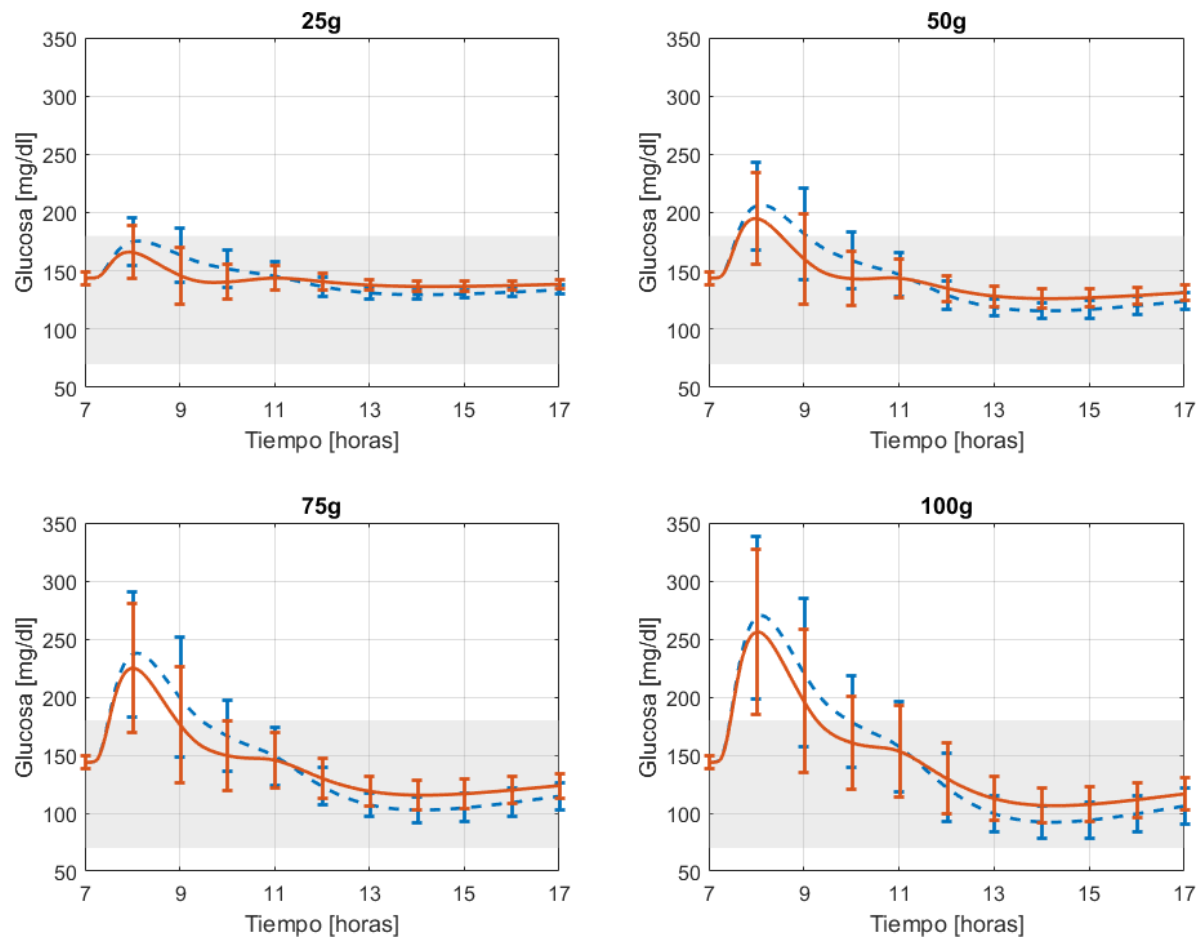

Figura 3.10: Respuesta de glucosa media \pm DE para los 10 adolescentes en el escenario de comida única. La línea azul a rayas corresponde al tratamiento estándar, la línea naranja sólida al SB automático. El área gris indica el rango euglucémico [70-180]mg/dl.

tiempo en la hipoglucemia ( $\mathrm{GP}<70 \mathrm{mg} / \mathrm{dl})$ (comida de $75 \mathrm{~g}: 5,92 \pm 14,48$ vs. $0,97 \pm 4,15$, $\rho=0,008$; comida de 100g: 9,5 $\pm 17,02$ vs. $1,85 \pm 7,05, \rho=0,002)$, el porcentaje de tiempo en $\mathrm{GP}<60 \mathrm{mg} / \mathrm{dl}$ (comida de 100g: 6,2 $\pm 14,31$ vs. $1,14 \pm 5,5, \rho=0,008$ ), y el porcentaje 

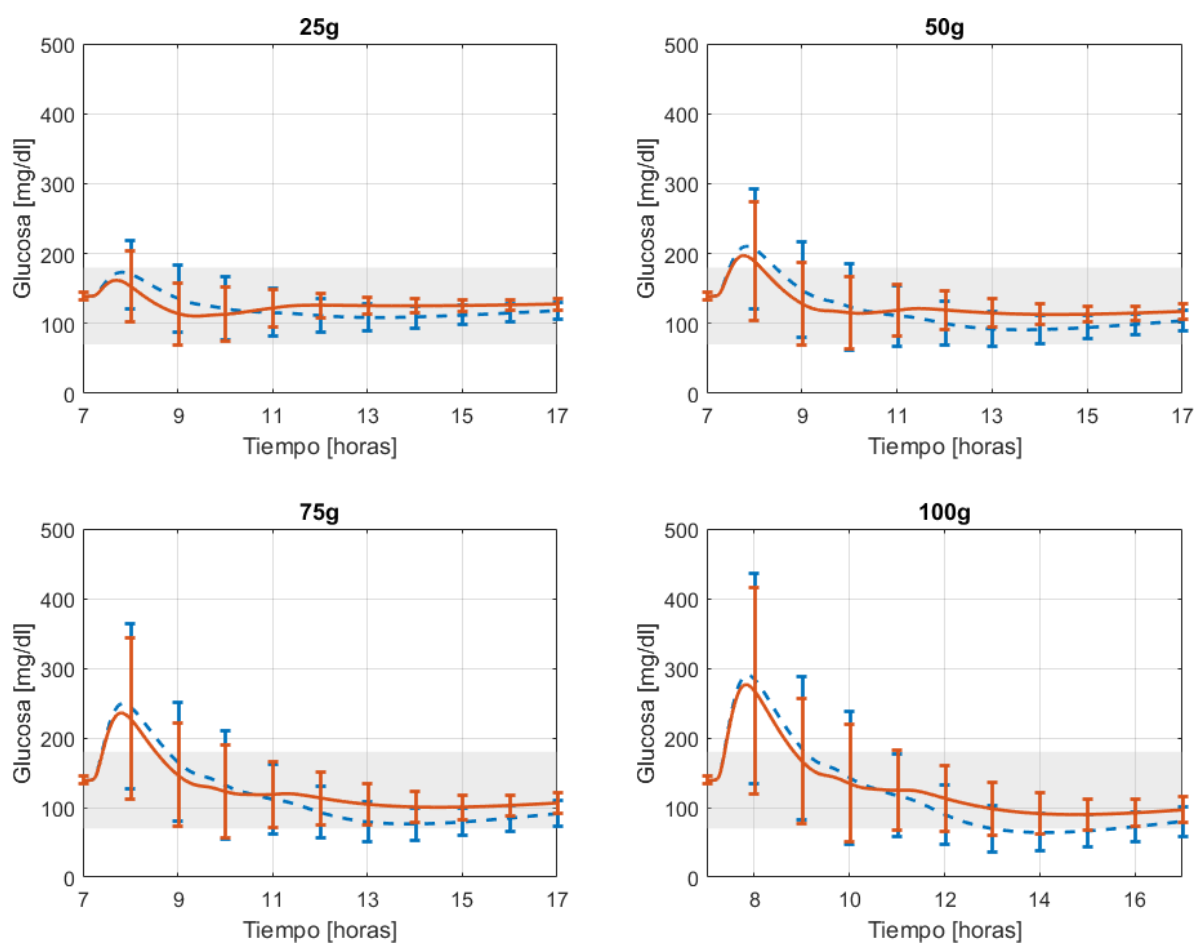

Figura 3.11: Respuesta media de glucosa \pm DE para los 10 niños en el escenario de comida única. La línea azul a rayas corresponde al tratamiento estándar, la línea naranja sólida al SB automático. El área gris indica el rango euglucémico [70-180]mg/dl.

de tiempo en hiperglucemia (GP> 180mg/dl) (comida de 25g: 3,37 $\pm 6,33$ vs. 1,96 $\pm 5,03$, $\rho=0,001$; comida de $50 \mathrm{~g}: 8,2 \pm 8,61$ vs. $6,05 \pm 8,31, \rho=0,001$; comida de $75 \mathrm{~g}: 12,18 \pm 9,94$ vs. $9,51 \pm 9,94, \rho<0,001$; comida de 100g: $15,38 \pm 11,26$ vs. $12,68 \pm 11,76, \rho<0,001)$. El resto de las métricas evaluadas muestran una tendencia de mejora aunque no demostraron un cambio estadísticamente significativo.

En las gráficas CVGA, representadas a partir de la Fig. 3.5 hasta la 3.8, se observa que la excursión de la glucosa se redujo bajo el SB automático en comparación con el tratamiento estándar (tratamiento estándar vs. SB automático; comida de 25g: Zona A $60 \%$ vs. $64 \%$; comida de $50 \mathrm{~g}$ : Zona A $27 \%$ vs. $37 \%$; comida de $75 \mathrm{~g}$ : Zona A $13 \%$ vs. $20 \%$; comida de 100g: Zona A $3 \%$ vs. $13 \%$ ). Se observa una tendencia de desplazamiento hacia abajo a la izquierda de la población total bajo el SB automático con respecto al tratamiento estándar y el SB manual, lo que se traduce a una mejora de la calidad de control. A pesar de que los tratamientos fallan en el control de glucosa de la población infantil para las comidas grandes en ciertos casos, los resultados indican que el tratamiento mediante el SB puede obtener una mejora en la calidad de control en lazo abierto sobre las comidas grandes respecto al tratamiento estándar. En las Figura 3.9] 3.11, donde se muestran los perfiles de glucosa para cada tamaño de comida, se puede ver cómo se reduce la excursión posprandial con el tratamiento del SB automático. El pico posprandial disminuyó y los pacientes alcanzan niveles de glucosa más cercanos a su nivel de ayuno en el posprandial tardío (8 horas después de la comida).

Hay que mencionar que el SB manual también logra una mejora importante con respecto al tratamiento estándar. Sin embargo, debe destacarse que los resultados obtenidos por el SB manual están correlacionados con el SB automático, ya que el ajuste del tiempo de corte para el SB manual proviene de la media de los tiempos de corte obtenidos por el método propuesto. Los resultados con el SB manual sólo se presentan en las gráficas CVGA por razones de claridad y espacio.

Los resultados de las simulaciones de los escenarios de un día se encuentran en el 


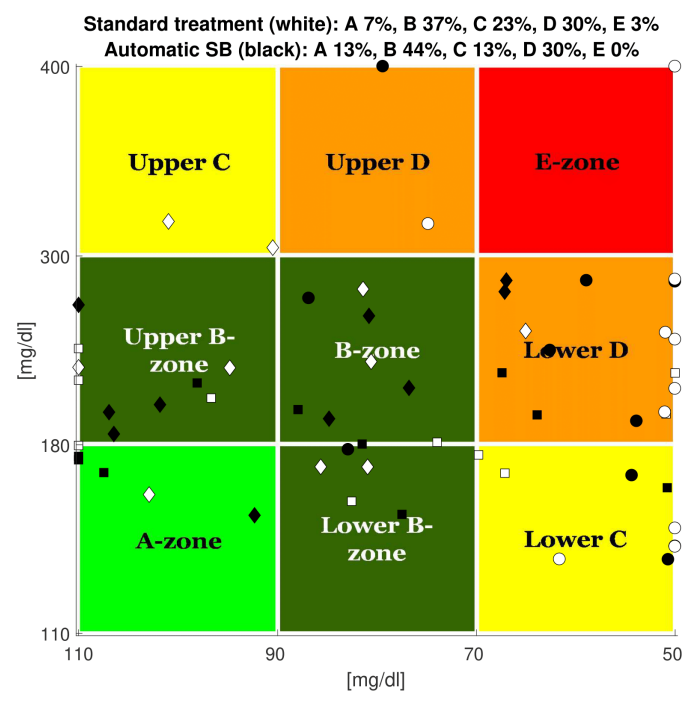

(a) Escenario 1

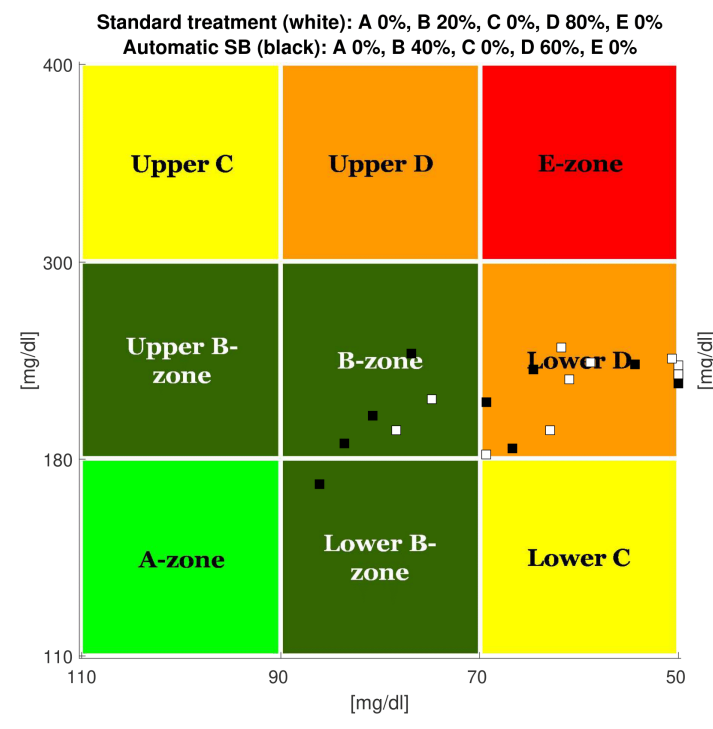

(b) Escenario 2

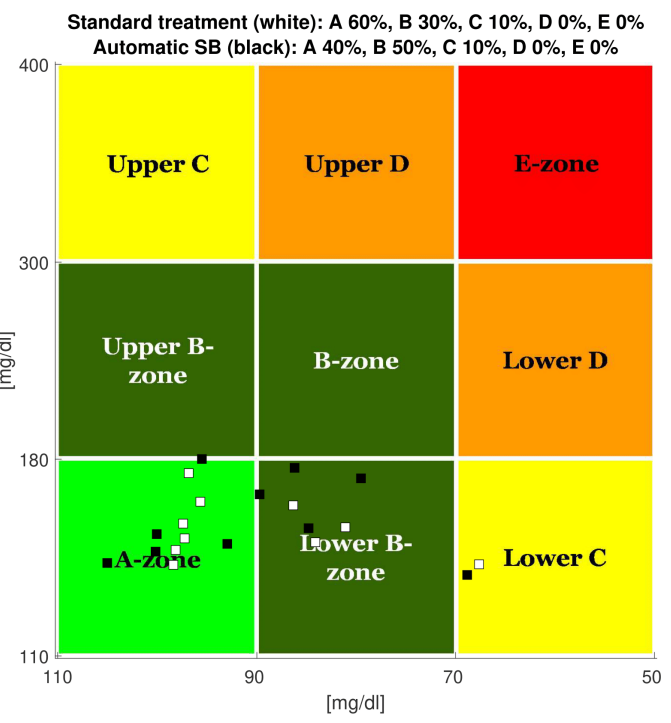

(c) Escenario 3

Figura 3.12: CVGA para los escenarios de un solo día. Comparación entre los tratamientos en adultos (marcadores cuadrados), adolescentes (marcadores romboidales) y niños (marcadores circulares).

cuadro 3.3. Puede observarse que en el caso del Escenario 1, el SB automático redujo de forma considerable el tiempo en hipoglucemia $(9,57 \pm 14,48$ vs. $4,21 \pm 6,18, \rho=0,028)$ y en hipoglucemia severa $(\mathrm{GP}<50 \mathrm{mg} / \mathrm{dl}: 3,79 \pm 7,52$ vs. $0,44 \pm 1,43, \rho=0,007 ; \mathrm{GP}<60 \mathrm{mg} / \mathrm{dl}$ : $6,37 \pm 11,65$ vs. $1,9 \pm 4,05, \rho=0,008)$. A su vez, se mejora el tiempo en rango euglucémico $(79,46 \pm 17,46$ vs. $86,29 \pm 11,73, \rho=0,007)$. El Escenario 2 muestra una mejora estadísticamente significativa en el porcentaje de tiempo transcurrido en euglucemia $(80,52 \pm 7,94$ vs. $86,23 \pm 9,2, \rho=0,001)$, hipoglucemia $(9,21 \pm 6,6$ vs. $5,99 \pm 6,48, \rho=0,004)$ y en hiperglucemia $(10,27 \pm 3,95$ vs. $7,78 \pm 4,92, \rho=0,001)$. Aunque los escenarios de un día tienen la misma cantidad total de carbohidratos, debido a la baja tasa de aparición de glucosa de las comidas BIG, no hay una mejora considerable en el Escenario 3. En la Fig. 3.13 se puede observar la reducción de la excursión de la glucosa, particularmente en los escenarios 1 y 2. Esta reducción gracias al SB automático se refleja en la Fig. 3.12 (Escenario 1: zona A $7 \%$ vs. $13 \%$, zona B $37 \%$ vs. $44 \%$; Escenario 2: zona B $20 \%$ vs. $40 \%$ ). En el caso de las comidas de BIG, el SB automático no muestra una mejora con respecto al tratamiento estándar. Vale la pena notar en la Fig. 3.13 (a) y (b) que el método propuesto conduce 


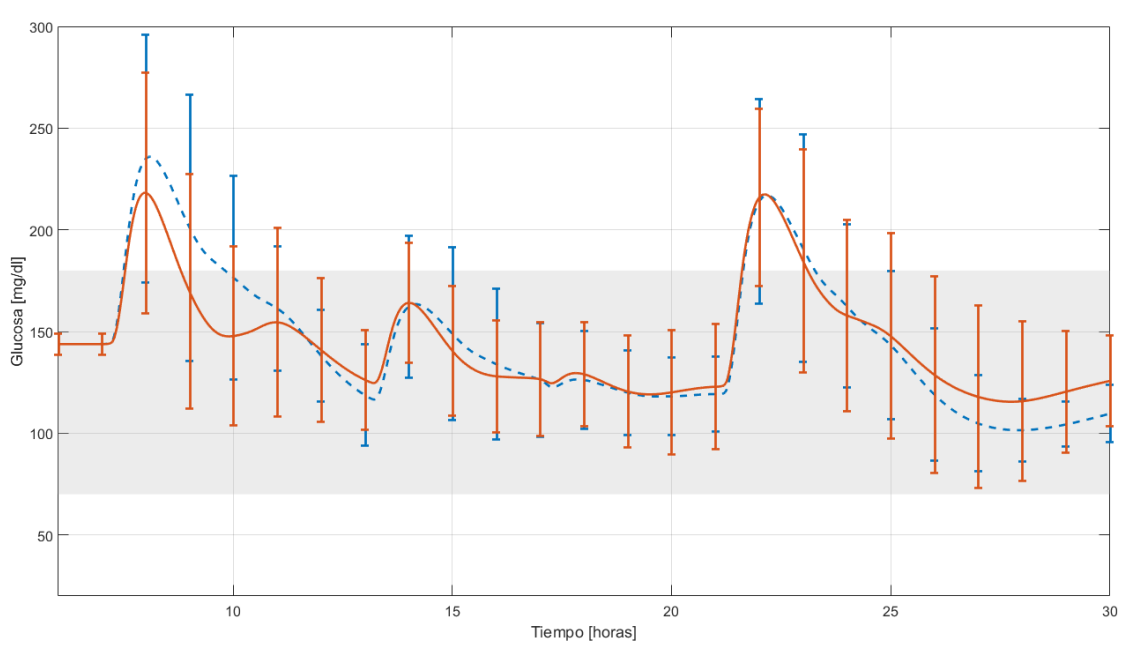

(a) Escenario 1

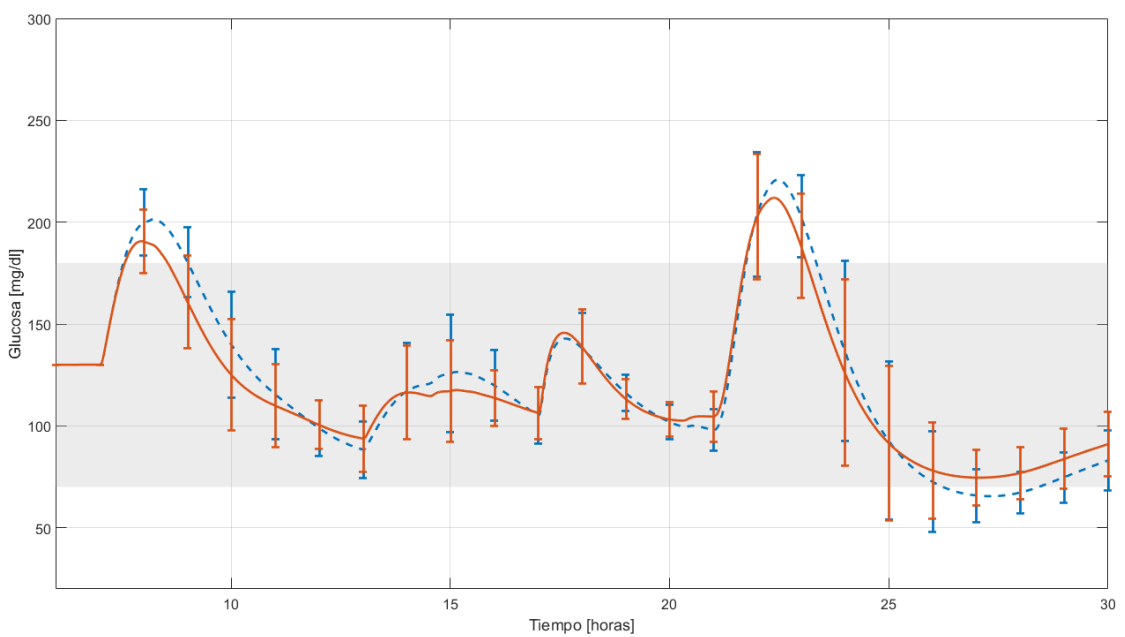

(b) Escenario 2

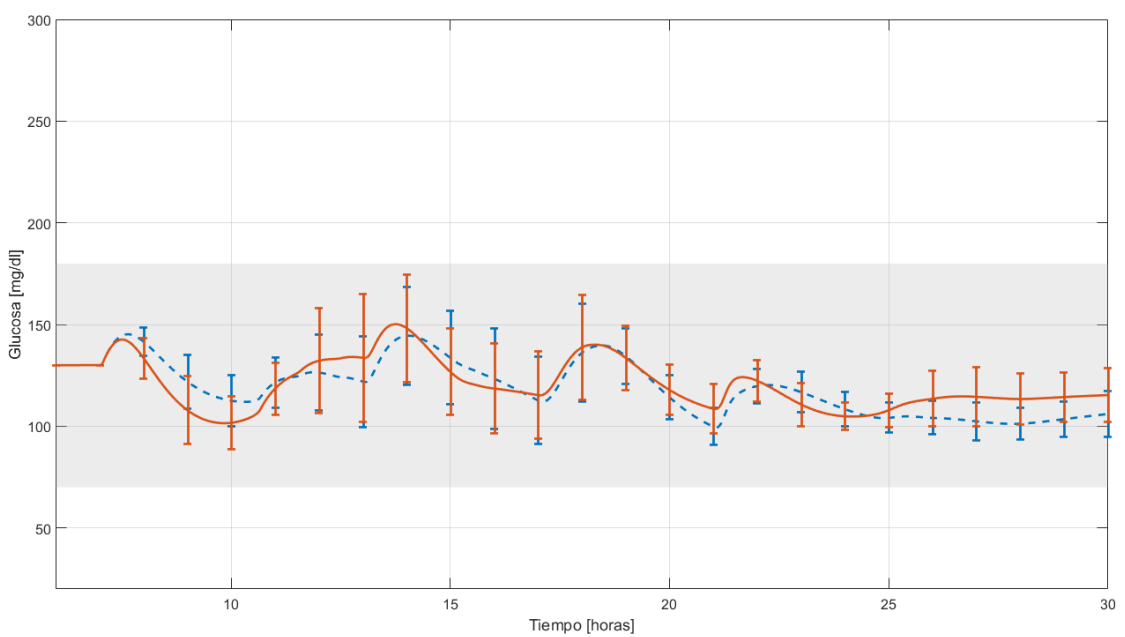

(c) Escenario 3

Figura 3.13: Respuesta media de glucosa \pm DE para los escenarios de un día. La línea azul a rayas corresponde al tratamiento estándar, la línea naranja sólida al SB automático. El área gris indica el rango euglucémico [70-180]mg/dl.

a niveles de glucosa más cercanos al objetivo durante la noche, después del período posprandial de la cena. Este resultado es buscado en el manejo de la glucosa en los sujetos 
con DMT1, debido al peligro que conlleva la hipoglucemia post-absortiva tardía nocturna. Se evaluó la robustez de la metodología en escenarios realísticos y los resultados indican que el método mejora el control de la glucosa, en particular para las comidas de AIG. Aunque la propuesta fue evaluada a lazo abierto, podrían usarse estimadores en tiempo real 137, 138 para así aplicar al SB automático basado en IOB al control de lazo híbrido, con el fin de mejorar el control de la glucemia.

\subsection{Control glucémico durante el ejercicio}

En Octubre de 2018 se realizó un estancia de investigación en la Universitat de Girona bajo la invitación del Dr. Josep Vehí, director del grupo de investigación "Modelado, Identificación e Ingeniería de Control" (MiceLab) del Institut d'Informàtica i Aplicacions. Vehí tiene una basta experiencia en el área vinculada al control de glucosa y sistemas de $\mathrm{PA}$. De hecho, fue a partir de estancias realizadas por los directores de tesis en la Universitat de Girona que se dio inicio al estudio del control automático de glucosa para sujetos con DMT1 en el grupo de trabajo del Instituto.

Durante el transcurso de la estancia, el grupo de trabajo de Vehí se encontraba realizando ensayos clínicos de su controlador sin anuncio de ejercicio [139]. Estos ensayos consistían en 3 intervalos de 15 minutos de ejercicio aeróbico de mediana intensidad en sujetos adultos. El controlador, además de regular el suministro de insulina, sugería el consumo de carbohidratos en caso de detectar una eventual hipoglucemia. El sujeto utilizaba un sensor Dexcom G5 y una bomba Dana $R$ y el lazo de control se cerraba mediante una plataforma montada en un smartphone con sistema operativo Android. A partir de entrevistarse con los sujetos, se tuvo noción de los efectos del ejercicio y el tratamiento implementado durante el mismo. Particularmente, el suministro de insulina basal a lazo-abierto se interrumpía 20 minutos antes y durante el ejercicio. Esto condujo a la observación de hiperglucemias tardías luego de la actividad física.

La limitación de la acción de control mediante restricciones en la estimación de IOB es un mecanismo que se instaló en el diseño de controladores de glucosa de lazo-cerrado [140,141]. Se han presentado reglas para determinar un límite superior en el nivel de IOB para el control posprandial [96] y durante el ejercicio [142]. Pero aún no se ha tenido en cuenta la implementación de un límite inferior. A continuación se presenta un enfoque de tratamiento a lazo-abierto durante el ejercicio que tiene en cuenta la estimación de IOB y una restricción suave en el límite inferior de insulina para evitar rebotes hiperglucémicos. Para la realización de los ensayos in-silico pertinentes de la metodología propuesta, se implementó una modificación al modelo utilizado en la plataforma UNLP. Esta modificación tiene en cuenta las dinámicas inducidas en la glucosa a causas de la actividad física, e intenta replicar el efecto de rebote en la glucosa.

\subsection{1. $\quad$ El ejercicio en la diabetes}

De acuerdo con la comunidad clínica, la Actividad Física $(\underline{\mathrm{AF}}$ es un componente importante en el manejo de la diabetes [143]. Aunque exhibe muchos beneficios para la salud, también presenta un desafío importante para el control glucémico de los sujetos. Adultos con DMT1 suelen obtener una mejora de su HbA1c presión, índice de masa corporal y pueden llegar a decrecer sus requerimientos diarios de insulina. A pesar de sus probados beneficios, el temor a las hipoglucemias y la pérdida ocasional del control glucémico alejan a los pacientes de la $\mathrm{AF}$ En la Fig. 3.14 se resume algunos beneficios, motivaciones y barreras que presenta el ejercicio físico a los sujetos diabéticos de acuerdo con [143, 144].

La $\mathrm{AF}$ generalmente se clasifica como aeróbica o anaeróbica, de acuerdo al sistema 
de energía utilizado para realizar la actividad. El ejercicio que utiliza sistema de energía aeróbica (quemar hidratos y grasas para obtener energía, para lo cual se necesita oxígeno) como caminar, andar en bicicleta, correr y nadar, incluye movimientos repetitivos y continuos que involucran grandes grupos de músculos. Los ejercicios de resistencia, generalmente con pesas, utilizan sistemas anaeróbicos, donde no se necesita oxígeno ya que la energía proviene de otras fuentes más inmediatas.

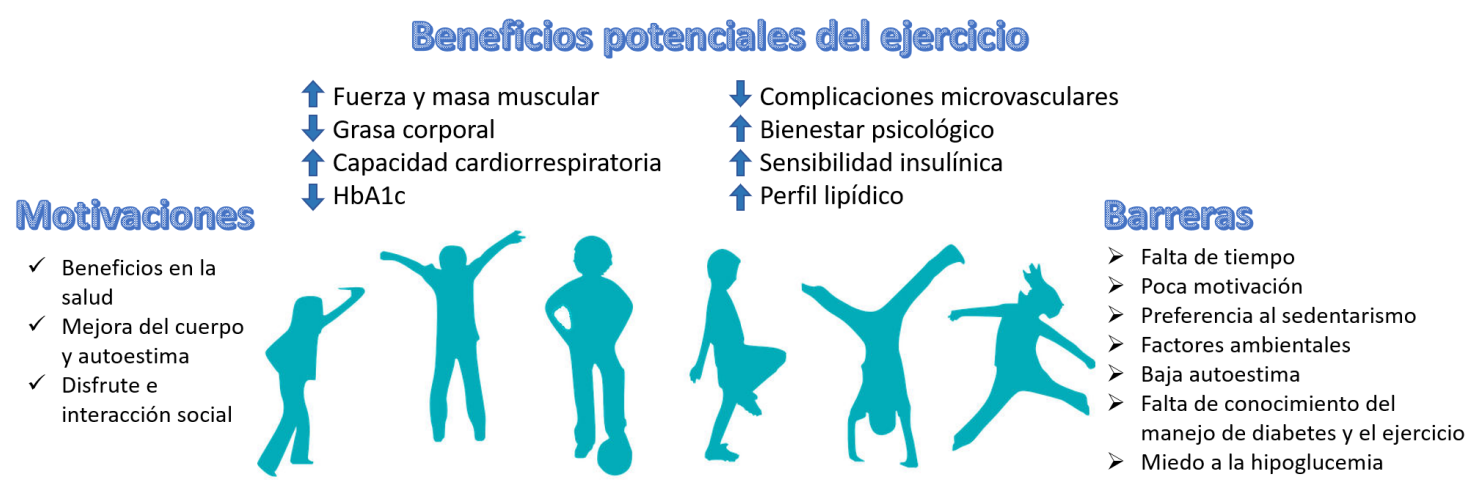

Figura 3.14: Beneficios, motivaciones y barreras de la actividad física en el manejo de la diabetes.

Las respuestas metabólicas son distintas de acuerdo a las diferentes formas de ejercicio (Fig. 3.15). Sin embargo, en casi todas ellas, independientemente de la intensidad o duración, las concentraciones de GP se mantienen normalmente dentro de un rango estrecho en personas sin diabetes. En particular, durante el ejercicio aeróbico, la secreción de insulina disminuye y la secreción de glucagón aumenta en la vena porta para facilitar la liberación de glucosa del hígado e igualar la tasa de absorción de glucosa en los músculos. En cambio, en sujetos con DMT1 la respuesta hormonal para mantener la homeostasis durante la AF cambia la dinámica de la glucosa del paciente [145] y diferentes clases de ejercicio pueden traer diferentes resultados. La respuesta de la GP en la DMT1 puede estar influenciada por la intensidad y duración de la $\mathrm{AF}$, locación del suministro de insulina, la cantidad de insulina en circulación, el nivel de GP antes del ejercicio, la composición de la última comida ingerida, entre otros 143. Esto afecta tanto el rendimiento de los tratamientos convencionales como el de los controladores de lazo cerrado.

Recientemente, Riddell et al. [143] presentaron una declaración de consenso que describe diferentes estrategias que pueden aplicarse a la terapia de insulina convencional para el control de la GP antes, durante y después del ejercicio. Generalmente, la GP cae durante la AF por lo que una de las intervenciones más comunes entre los sujetos con DMT1 durante la $\mathrm{AF}$ es la reducción o suspensión de la insulina basal para evitar una posible hipoglucemia (Delayed-Onset Hypoglycemia D'OH [128). Cuando se consideran largos períodos de AF la suspensión total de insulina puede conducir a niveles bajos de insulina en el plasma. La hipoinsulinemia no permiten que el músculo use la glucosa adecuadamente, y esto sumado al efecto de la Producción Endógena de Glucosa (PEG), conduce al aumento de concentración de GP [146] luego de determinado tiempo, a lo que suele referirse como "rebote" (Fig. 3.16).

La hiperglucemia posterior al ejercicio es un aspecto al que una gran parte de la comunidad clínica no le brinda la suficiente importancia [147]. Sin embargo, algunos autores resaltan que la hiperglucemia asociada a la $\mathrm{AF}$ puede deberse a una reducción agresiva de la insulina u omisión total de la misma, además de un consumo excesivo de carbohidratos debido al temor a la hipoglucemia 144,148 . Además, dentro de los beneficios de predecir las respuestas glucémicas en la $\mathrm{AF}$ destacan la importancia en los cambios en el régimen 


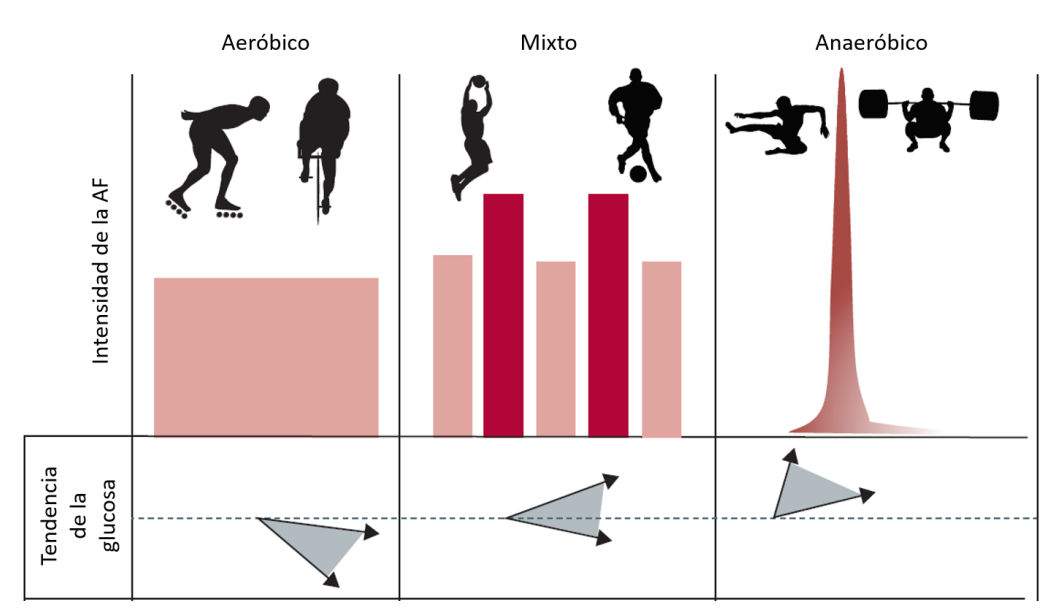

Figura 3.15: Existe una alta variabilidad individual en las respuestas de la glucosa en sangre a las diferentes formas de ejercicio, como lo indica la flechas y sombreado gris. En general, el ejercicio aeróbico disminuye la glucemia, el ejercicio anaeróbico aumenta la glucemia, y las actividades mixtas están asociadas con la estabilidad de la glucosa. Las respuestas individuales dependen de varios factores adicionales, incluyendo la duración e intensidad de la actividad, las concentraciones iniciales de glucosa en sangre, el estado físico individual, concentraciones de insulina, glucagón y otras hormonas contrareguladoras en circulación, y el estado nutricional de la persona 143 .

y en el desarrollo de tratamientos para prevenir o corregir la hiperglucemia.

\subsubsection{Modelos para la representación del ejercicio}

Debido a la complejidad de desarrollar y probar nuevos tratamientos para el manejo de la diabetes se recurre a ensayos in-silico sobre modelos matemáticos. Pocos modelos matemáticos que desplieguen los efectos del ejercicio en la DMT1 se han propuesto hasta la fecha. Algunos de ellos, por ejemplo, son los modelos propuestos por Breton et al. [85], Derouich et al. [149] y Dalla Man et al. [150]. En particular, Derouich et al. combinaron un modelo para la cinética de la glucosa, con un modelo perturbado en el que tres parámetros tienen en cuenta el efecto del ejercicio físico. Representan la aceleración de la utilización del glucosa y el aumento de la sensibilidad a la insulina por parte de los músculos y el hígado. La eficacia de este modelo ha sido confirmada por los resultados de Breton. El modelo propuesto por Breton relaciona las modificaciones en la acción de la insulina y la respuesta de la glucosa a los cambios en la frecuencia cardíaca. En un trabajo posterior, Dalla Man et al. propuso y probó tres posibles extensiones de este último estudio. Posteriormente en Schiavon et al. [151] se propone un modelo más sencillo, el cual modifica la SI mediante un parámetro variante en el tiempo aplicado sobre el modelo de utilización de glucosa dependiente de la insulina. Este modelo es utilizado para la optimización del suministro basal durante el ejercicio de forma in-silico.

Por otro lado, Roy y Parker [66 desarrollaron una extensión del modelo mínimo de Bergman al agregar la dinámica inducida por el ejercicio de la insulina plasmática y la elevación de la captación de glucosa y las tasas de PEG. Su modelo representa la dinámica de la glucosa y la insulina durante el ejercicio a corto y largo plazo, siendo consistente con los datos de la literatura. Su ventaja se basa en proporcionar una descripción cuantitativa de la $\mathrm{AF}$ en términos de la intensidad relativa del ejercicio expresada como porcentaje del oxígeno máximo $\left(V_{2}^{\max }\right.$.). Aún así, el modelo de Bergman es una representación simple del sistema insulina-glucosa y no posee las dinámicas más complejas que contiene el modelo de Dalla Man, por lo que representa una limitación a la hora de ensayar estrategias de control glucémico de forma in-silico.

La limitación que comparten los modelos encontrados en la literatura parte del efecto que intentan representar. Primordialmente, buscan reflejar las caídas en la GP debido 


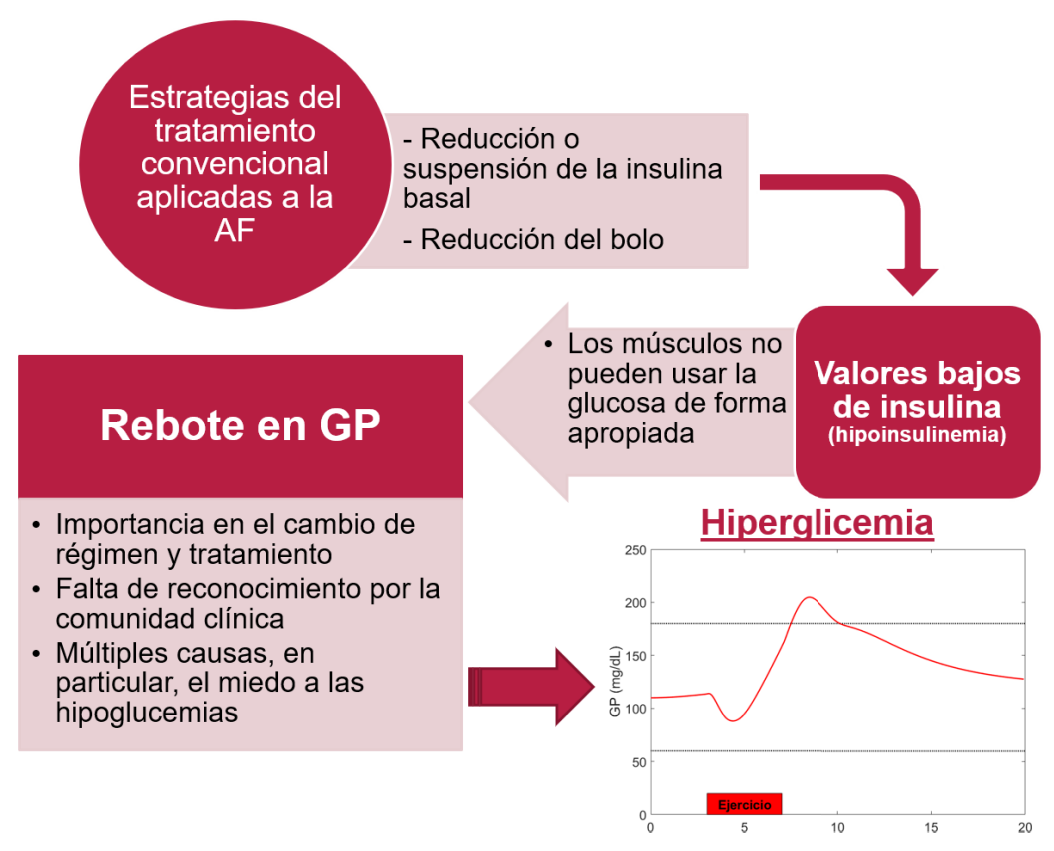

Figura 3.16: Motivos por los que se produce el efecto de rebote en la GP luego o durante el ejercicio en la DMT1

al aumento en la SI, con el fin de utilizarse en el desarrollo de métodos de prevención de hipoglucemias durante el ejercicio. El efecto descripto anteriormente como "rebote" no puede ser reproducido adecuadamente. A partir de esta inquietud, a continuación se busca, en primer lugar, representar el fenómeno de rebote en la GP observado tanto en los trabajos anteriormente mencionados como clínicamente. Para esto, se propone modificar el modelo utilizado en el simulador UVA/Padova considerando la propuesta de Roy y Parker, con el objetivo de desplegar los efectos en la PEG, los cambios en la SI y la utilización de glucosa por los tejidos durante el ejercicio. En segundo lugar, se propone un tratamiento de mitigación de hiperglucemias debido al fenómeno de rebote. El mismo consiste en la manipulación de la insulina basal de modo que se asegure un mínimo de insulina disponible en el cuerpo, mediante la estimación de IOB. Este enfoque puede ser aplicado en la tecnología actualmente existente sin aportar complejidad adicional a la misma. Finalmente, se evalúa de forma in-silico tanto la representación del efecto de rebote por el modelo modificado como el desempeño del tratamiento propuesto.

\section{Modelo propuesto por Dalla Man et al.}

El trabajo publicado por Dalla Man et al. [150] merece una mención especial ya que fue propuesto poco después del modelo enfocado a las comidas que derivó en el simulador UVA/Padova [74]. El enfoque utilizado en este trabajo, y el que suele utilizarse en general, es el de definir el ejercicio como una perturbación que incrementa la utilización de glucosa por medio del aumento de la SI. Este perturbación actúa durante y después de que se produzca la actividad física, exhibiendo los efectos que ésta tiene sobre la dinámica de la glucosa incluso después de haber cesado. Aquí se relaciona el ritmo cardiaco Heart Rate (프) del sujeto con un cambio en la acción de la insulina sobre la glucosa. Se considera que el HR se asocia a la duración e intensidad del ejercicio. El modelo se encuentra definido por las siguientes ecuaciones:

$$
\dot{Y}_{i}(t)=-\frac{1}{T_{H R}}\left[Y_{i}(t)-\left(H R(t)-H R_{b}\right)\right], \quad Y_{i}(0)=0
$$




$$
\begin{gathered}
\dot{Z}_{i}(t)=-\left(\frac{f\left(Y_{i}(t)\right.}{T_{i n}}-\frac{1}{T_{e x}} Z_{i}(t)\right)+f\left(Y_{i}(t)\right), \quad Z_{i}(0)=0 \\
W_{i}(t)= \begin{cases}\int_{0}^{t}\left(H R(t)-H R_{b}\right) d t & \text { sit }<t_{z} \\
0 & \text { casocontrario }\end{cases} \\
f\left(Y_{i}(t)\right)=\frac{\left(\frac{Y_{i}(t)}{a \cdot H R_{b}}\right)^{n}}{1+\left(\frac{Y_{i}(t)}{a \cdot H R_{b}}\right)^{n}}
\end{gathered}
$$

donde $H R_{b}$ es el ritmo cardíaco basal y $H R(T)$ es la señal que regula diferentes niveles de ejercicio. $Y_{i}(t)$ es la señal retardada de la elevación de HR por sobre la basal, $Z_{i}(t)$ es la señal que afecta de forma no-lineal al uso de glucosa dependiente de la insulina y $W_{i}(t)$ es el área bajo la curva, que tiene en cuenta intensidad y duración del ejercicio. Este modelo afecta a la utilización de la glucosa dependiente de la insulina (ec. (2.24) ) teniendo en cuenta la duración e intensidad del ejercicio de la siguiente forma:

$$
U_{i d}(t)=\frac{V_{m 0}(1+\beta \cdot Y(t))+V_{m x}(1+\alpha \cdot Z(t) \cdot W(t)) \cdot\left(X(t)+I_{b}\right)-V_{m x} \cdot I_{b}}{K_{m 0}\left[1-\gamma \cdot Z(t) \cdot W(t) \cdot\left(X(t)+I_{b}\right)\right]+G_{t}(t)} .
$$

Debe notarse que esta modificación se realizó antes de que se introdujera la nueva versión del modelo 75 que considera cambios en $U_{i d}$ en el rango hipoglucémico. Este modelo ha sido utilizado en algunos trabajos para ensayos in-silico de controladores de lazocerrado [142], pero no ha sido validado clínicamente.

En un comienzo, se implementó este modelo en la plataforma de simulación UNLP con el objetivo era reproducir el efecto "rebote" por medio de ajuste de parámetros. Se consideraron diferentes enfoques a partir de lo discutido y estudiado de la literatura del metabolismo durante el ejercicio. Se tuvieron en cuenta cambios en la secreción de glucagón (Capítulo 2 Sección 2.1.4, ec. (2.31)), en la producción endógena de glucosa (ec. (2.17)), y el efecto durante y luego del ejercicio (función $\mathrm{Z}(\mathrm{t})$ ). A pesar de numerosos ensayos mediante el método de Monte Carlo para conseguir la respuesta esperada, esta metodología no permitió conseguir los resultados deseados.

\section{Modelo propuesto por Schiavon et al.}

En el trabajo presentado por Schiavon et al. se analiza cual es la infusión óptima de insulina basal durante el ejercicio. Como se mencionó anteriormente, en la terapia estándar de lazo-abierto, los sujetos generalmente, reducen sus tasas de infusión de insulina basal para prevenir el riesgo de hipoglucemia debido al rápido cambio en la sensibilidad a la insulina causado por el ejercicio, como se ejemplifica en la Fig. 3.17. En la terapia de lazo-cerrado, la necesidad de informar a los algoritmos de control con respecto a la AF inminente para reducir apropiadamente las tasas de infusión de insulina y prevenir la hipoglucemia sigue siendo discutida 151 .

El enfoque utilizado en [151] para representar la AF consiste en afectar la SI durante y una vez terminada la misma. Proponen un parámetro variante en el tiempo $\alpha$, el cual corresponde a una ganancia que afecta al parámetro $V_{m x}$ de la ecuación (3.35). El valor de dicho parámetro es igual a la unidad antes del ejercicio, y cambia en forma de escalón a un valor dado durante el transcurso de la $\mathrm{AF}$ (ver Fig. 3.17). Una vez finalizado el ejercicio vuelve a la unidad con una extrapolación lineal de duración $T_{\alpha}$. A partir de valores clínicos, se ajustaron los parámetros a $\alpha=3,29$ y $T_{\alpha}=180$ 152. 


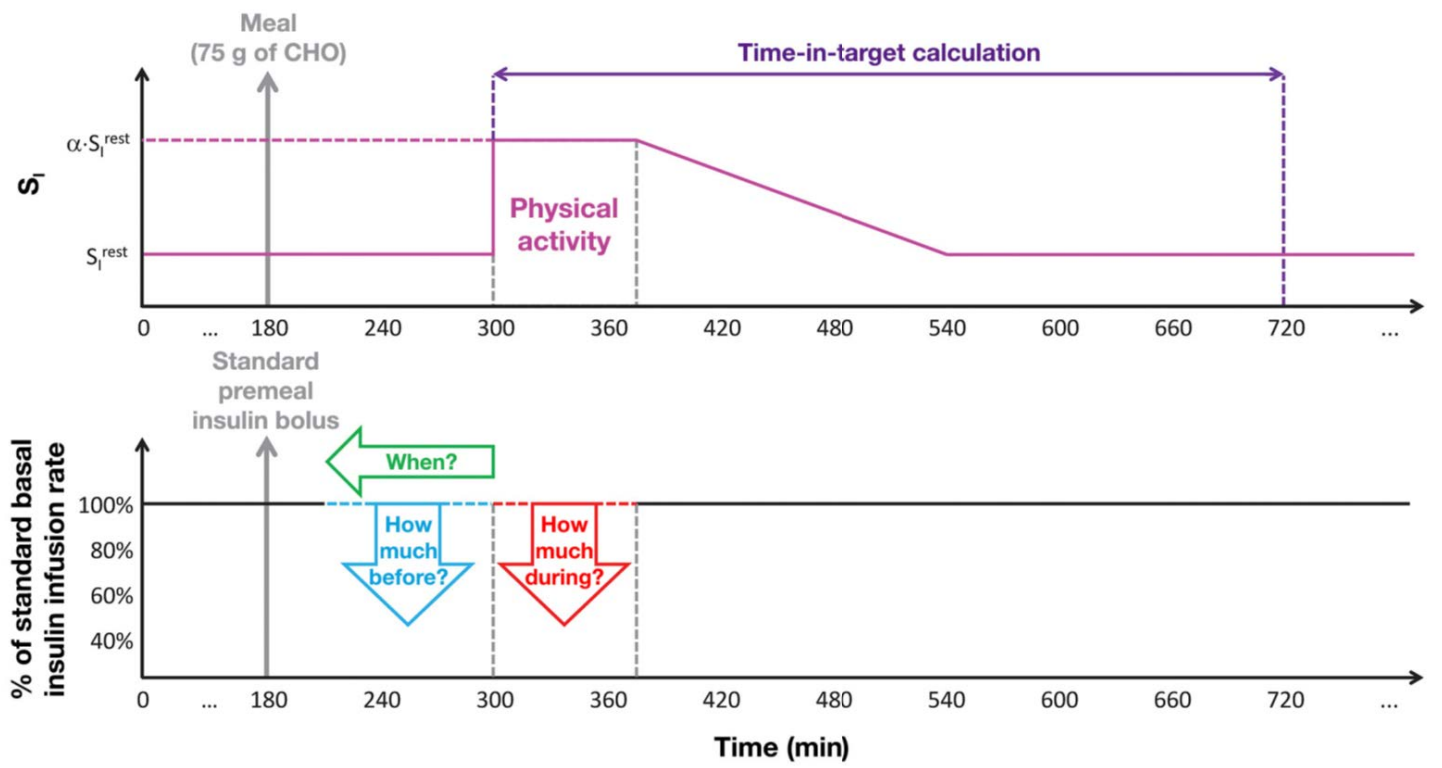

Figura 3.17: En el panel superior se ejemplifica el cambio en la SI durante la simulación que imita el protocolo experimental reportado por Schiavon et al. 152 durante la sesión de ejercicio (línea rosa). Debajo se muestra el bolo de insulina estándar antes de las comidas y ajustes de la tasa de infusión de insulina basal, con respecto al perfil del paciente, durante (flecha roja) y/o antes (flechas azul y verde) de la sesión de ejercicios 151 .

\section{Modelo propuesto por Roy y Parker}

Roy y Parker presentan la ampliación del modelo mínimo desarrollado previamente para incluir los principales efectos del ejercicio en los niveles de glucosa e insulina en plasma. Se desarrollaron ecuaciones diferenciales para captar la dinámica inducida por el ejercicio de la depuración de la insulina en plasma y la elevación de la captación de glucosa y de los índices de producción de glucosa hepática. La disminución de la producción de glucosa hepática resultante del ejercicio prolongado se modeló mediante una ecuación que depende de la intensidad y la duración del ejercicio [66].

Para representar la intensidad del ejercicio, se utiliza la tasa máxima de consumo de oxígeno para un individuo, dado por $\mathrm{VO}_{2}^{\max }$. Se considera aproximadamente proporcional al consumo de oxígeno con el gasto de energía, por lo que se puede medir indirectamente la capacidad máxima para el ejercicio aeróbico de un individuo midiendo su consumo de oxígeno. Si se expresa la $\mathrm{AF}$ en términos de porcentaje de $V \mathrm{O}_{2}^{\max }$ (el $\mathrm{PVO}_{2}^{\max }$ ) puede compararse los efectos de la $\mathrm{AF}$ entre individuos de mismo sexo y peso similares. Se considera que en estado basal el $\mathrm{PVO}_{2}^{\max }$ de una persona es del $8 \%$ e incrementa rápidamente una vez comenzada la $\mathrm{AF}$, alcanzando su valor definitivo a los 5-6 minutos de ejercicio y manteniéndose constante durante el mismo. En [66] se propone la siguiente ecuación diferencial para el $P V O_{2}^{\max }$ :

$$
P \dot{V} O_{2}^{\max }(t)=-0,8 P V O_{2}^{\max }(t)+0,8 u_{e x}(t) P V O_{2}^{\max }(0)=0
$$

donde $u_{e x}(t)$ es la intensidad final del ejercicio por sobre el estado basal, correspondiente al $8 \%$ del $P V O_{2}^{\max }$.

La AF promueve la caída de la concentración de insulina en plasma, eleva la toma de glucosa por los tejidos y aumenta la liberación de glucosa de parte del hígado. Roy y Parker proponen ampliar el modelo mínimo de Bergman de acuerdo a las siguientes 
dinámicas:

$$
\begin{array}{rlrl}
\dot{I}(t) & =-n I(t)+p_{4} u_{1}(t)-I e(t) & I(0)=I_{b}, \\
\dot{X}(t) & =-p_{2} X(t)+p_{3}\left[I(t)-I_{b}\right] & & X(0)=0, \\
\dot{G}(t) & =-p_{1}\left[G(t)-G_{b}\right]-X(t) G(t) & \\
& +\frac{W}{V o l_{G}}\left[G-\operatorname{prod}(t)-G_{\text {gly }}(t)-G_{\text {up }}(t)\right]+u_{2}(t) & & G(0)=G_{b} \\
\dot{G}_{\text {prod }}(t) & =a_{1} P V O_{2}^{\max }(t)-a_{2} G_{\text {prod }}(t) & & G_{\text {prod }}(0)=0, \\
\dot{G}_{\text {up }}(t) & =a_{3} P V O_{2}^{\max }(t)-a_{4} G_{\text {up }}(t) & G_{\text {up }}(0) & =0, \\
\dot{I}_{e}(t) & =a_{5} P V O_{2}^{\max }(t)-a_{4} I_{e}(t) & I_{e}(0) & =0,
\end{array}
$$

donde la variable $G_{u p}(t)(\mathrm{mg} / \mathrm{kg} / \mathrm{min})$ representa la toma de glucosa durante el ejercicio, $G_{\text {prod }}(t)(\mathrm{mg} / \mathrm{kg} / \mathrm{min})$ es la producción hepática e $I_{e}(t)(\mu \mathrm{U} / \mathrm{ml} / \mathrm{min})$ es la tasa de eliminación de insulina del sistema circulatorio debido al ejercicio. A su vez, se tiene en cuenta la variable $G_{g l y}(t)(\mathrm{mg} / \mathrm{kg} / \mathrm{min})$ la cual representa la caída de la tasa de glicogenólisis durante el ejercicio prolongado debido al agotamiento de las reservas de glicógeno. Esta última empieza a decrecer cuando el gasto de energía excede un valor umbral $A_{T H}$, interpolado respecto a la intensidad del ejercicio como:

$$
A_{T H}=-1,152\left[u_{e x}(t)\right]^{2}+87,471 u_{e x}(t) .
$$

Entonces, la glicogenólisis durante el ejercicio prolongado es representada de la siguiente forma:

$$
\dot{G}_{g l y}(t)=\left\{\begin{array}{cl}
0 & A(t)<A_{T H} \\
k & A(t) \geq A_{T H} \\
-\frac{G_{g l y}}{T_{1}} & u_{e x}(t)=0
\end{array},\right.
$$

donde $A(t)$ es la integración de la intensidad del ejercicio $u_{e} x(t)$, calculada por:

$$
\dot{A}(t)=\left\{\begin{array}{cc}
0 & u_{e x}(t)>0 \\
-\frac{A(t)}{0,001} & u_{e x}(t)=0
\end{array} .\right.
$$

Los parámetros $a_{i}$ fueron ajustados y validados con datos clínicos de pacientes sanos y con DMT1, En la tabla 3.4 se muestran los valores medios y sus unidades.

Tabla 3.4: Valores medios de los parámetros de las dinámicas propuestas por Roy y Parker.

\begin{tabular}{|c|c|c|}
\hline Parámetro & Valor medio & Unidad \\
\hline$a_{1}$ & 0,00158 & $\mathrm{mg} / \mathrm{kg} \cdot \mathrm{min}^{2}$ \\
\hline$a_{2}$ & 0,056 & $1 / \mathrm{min}$ \\
\hline$a_{3}$ & 0,00195 & $\mathrm{mg} / \mathrm{kg} \cdot \mathrm{min}^{2}$ \\
\hline$a_{4}$ & 0,0485 & $1 / \mathrm{min}$ \\
\hline$a_{5}$ & 0,00125 & $\mu \mathrm{U} / \mathrm{ml} \cdot \mathrm{min}$ \\
\hline$a_{6}$ & 0,075 & $1 / \mathrm{min}$ \\
\hline $\mathrm{k}$ & 0,0108 & $\mathrm{mg} / \mathrm{kg} \cdot \mathrm{min}^{2}$ \\
\hline$T_{1}$ & 6 & $\mathrm{~min}$ \\
\hline
\end{tabular}

\subsubsection{Propuesta de modelo}

Durante la participación en los ensayos clínicos que se estaban realizando en Barcelona, se tuvo la oportunidad de profundizar aspectos del metabolismo durante el ejercicio con 


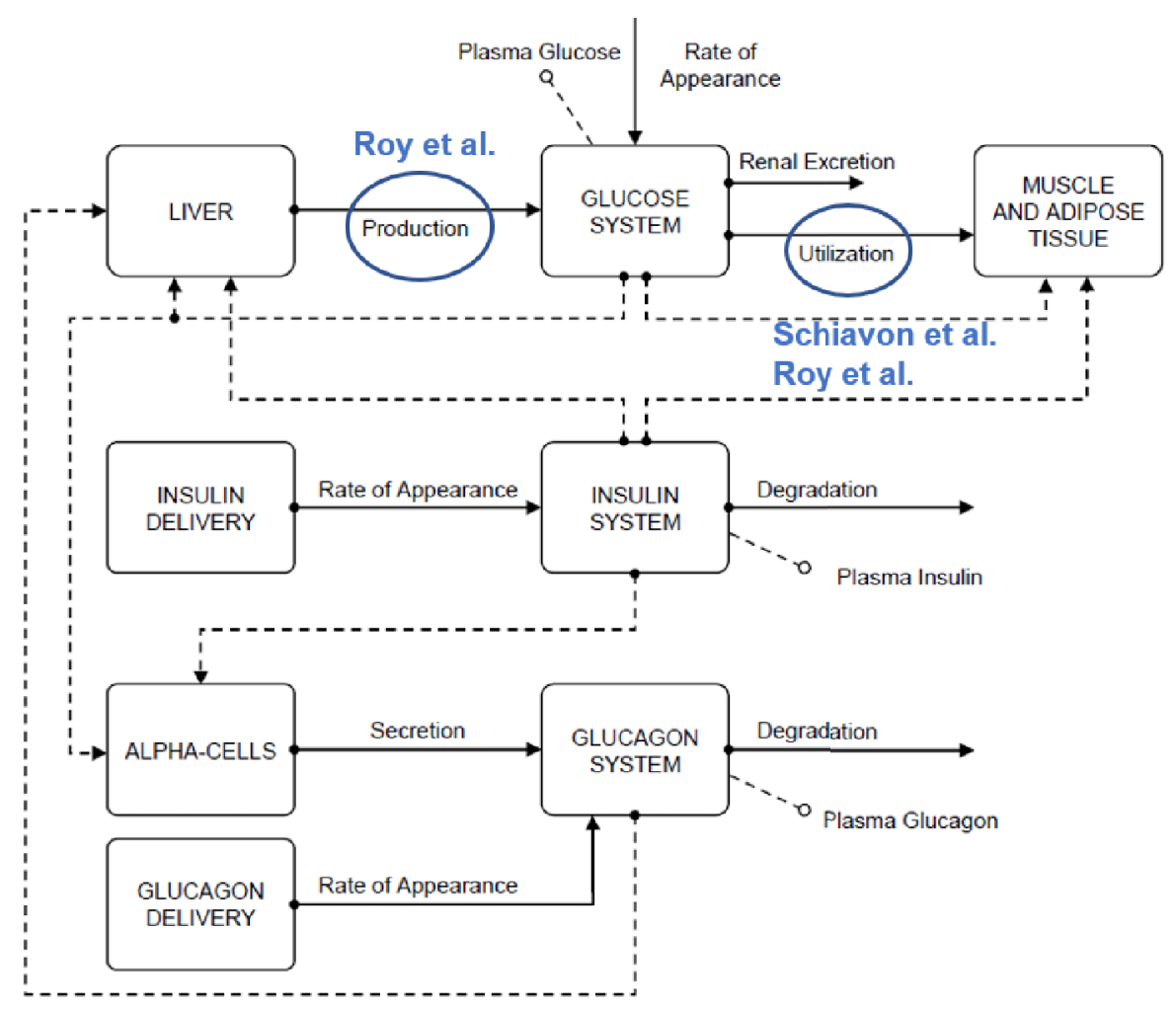

Figura 3.18: Representación en bloques del modelo UVA/Padova 75 con remarques en las dinámicas que se afectan durante el ejercicio mediante lo propuesto en 66,151$]$.

el médico deportólogo especialista en diabetes Serafín Murillo [153], lo que motivo la propuesta que se presenta a continuación.

Para reproducir el efecto de "rebote" en la GP, se parte del modelo utilizado en la plataforma UVA/Padova [75] y se lo amplía a partir de la propuesta de Roy y Parker 66 y Shciavon et al. [151]. En la Fig. 3.18 se puede visualizar el diagrama de bloques del modelo de Dalla Man y se señalan los bloques modificados.

El efecto principal de la $\mathrm{AF}$ consiste en el aumento de la utilización de la glucosa por parte de los tejidos. El modelo de utilización de la glucosa actualmente implementado en el simulador asume que la cinética de la glucosa se describe por dos compartimentos. La utilización de la glucosa debido al cerebro e independiente de la insulina, es considerada constante, y la utilización de la glucosa dependiente de la insulina $\left(U_{i d}\right)$, tiene lugar en el compartimiento remoto y depende de forma no lineal de la glucosa en los tejidos:

$$
U_{i d}=\frac{\left[V_{m 0}+V_{m x} \alpha(t) X(t)\left(1+r_{1} r i s k\right)\right] G_{t}(t)}{K_{m 0}+G_{t}(t)}+G_{u p},
$$

siendo $G_{t}(t)$ es la masa de glucosa en el compartimiento periférico; $V_{m 0}, V_{m x}, K_{m 0}$ y $p_{2 U}$ son parámetros del modelo, con $V_{m x}$ en particular representando la sensibilidad a la insulina, es decir, la capacidad de la insulina para estimular la utilización de la glucosa. La variable $X(t)$ corresponde a la acción de la insulina sobre la utilización de la glucosa.

Otra dinámica a tener en cuenta es la ecuación que gobierna la producción endógena de glucosa, la cual es afectada por la concentración de insulina que circula por el hígado $X^{L}(t)$, la concentración de GP $G_{p}(t)$ y la de glucagón $X^{H}(t)$ :

$$
P E G(t)=k_{p 1}-k_{p 2} G_{p}(t)-k_{p 3} X^{L}(t)+\zeta X^{H}(t)+\left[G_{\text {prod }}-G_{\text {gly }}\right] k_{\text {adapt }}
$$

Aquí se optó por agregar las dinámicas propuestas por Roy y Parker, de manera de modificar la PEG y la utilización de glucosa. La nuevas dinámicas de utilización de glucosa, 

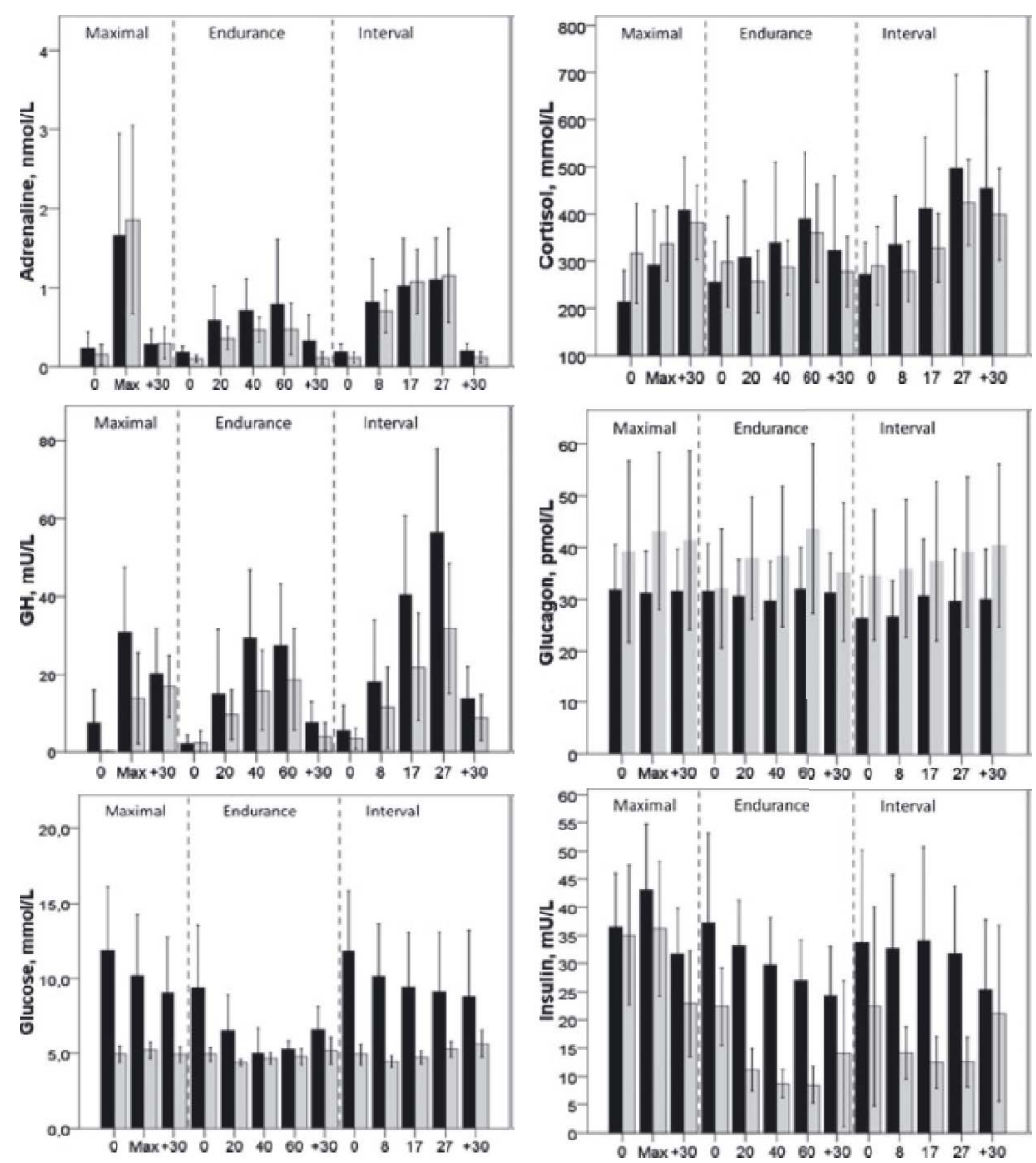

Figura 3.19: Hormonas y metabolitos medidos antes, durante y después de diferentes intensidades de ejercicio en adolescentes con diabetes $(n=12$, negro) y controles $(n=12$, gris $) 145$.

producción endógena y la caída de glicógeno se superponen a las dinámicas dadas por el modelo de Dalla Man. El término $G_{u p}$ se agrega a la ec. (3.35), mientras que $G_{p r o d}-G_{g l y}$ se agrega a la ec. (3.36) mediante una ganancia de adaptación. El efecto de las mismas puede gobernarse gracias a la entrada $u_{e x}(t)$ dadas las dinámicas por las ecuaciones (3.25), (3.29) y 3.30 .

Considerando el resultado principal de Adolfsson et al. 145], el cual refleja que los sujetos diabéticos y los sanos tienen una capacidad física y una respuesta hormonal similares (Fig. 3.19. Los paneles muestran los niveles de adrenalina (A), cortisol (B), hormona del crecimiento $(\mathrm{GH})(\mathrm{C})$, glucagón $(\mathrm{D})$, glucosa $(\mathrm{E})$ e insulina $(\mathrm{F})$ durante diferentes pruebas e ejercicio (intensidad máxima, de resistencia y de intervalo). Las barras representan la media y la confianza intervalo (95\%). Se observaron diferencias entre los adolescentes con diabetes y los controles en el nivel máximo medio de glucosa e insulina y el área bajo la curva de la glucosa y la insulina. Además de esto, las respuestas hormonales no difieren entre los grupos, excepto por el nivel medio de GH máximo durante la prueba del intervalo. Se puede inferir que la respuesta hormonal en el sistema glucosa-insulina podría omitirse en principio al modelar el efecto de la $\mathrm{AF}$ en la DMT1 sin perder validez.

Con la modificación de modelo propuesta se busca representar una mayor variabilidad de respuestas durante el ejercicio. En particular, se buscó representar el efecto de rebote que es descripto tanto por pacientes como por los clínicos y no es lo suficientemente reconocido. Las simulaciones que se muestran en la sección 3.3.5 muestran que el modelo porpuesto permite obtener una amplia banda de excursiones, la cual dispone de suficientes parámetros de ajuste como para representar datos clínicos.

Una vez montado y ajustado el modelo modificado, se propone una alternativa al tra- 
tamiento convencional. Esta consiste en la suspensión de insulina basal durante el ejercicio siempre que el nivel de IOB no caiga por debajo de un valor determinado, de forma tal de contrarrestar la hiperglucemia debido al efecto "rebote", pero aún así previniendo una posible hipoglucemia debido a la AF.

\subsubsection{Mitigación del efecto rebote}

Muchos clínicos y autores recomiendan a sujetos con DMT1 bajo la terapia CSII una reducción del suministro basal del 50-90\% entre 60-90 minutos antes del ejercicio hasta que se detenga el mismo, o incluso la suspensión total durante el mismo 143, 144]. Una suspensión prolongada del suministro de insulina basal conduce a la hipoinsulinemia, la cual puede llevar a complicaciones como la hiperglucemia o la cetoacidosis. La hiperglucemia durante el ejercicio es un factor no lo suficientemente reconocido por la comunidad clínica y no se tiene lo suficientemente en cuenta a la hora de proponer guías de tratamiento para realizar $\mathrm{AF}$ [147]. El enfoque propuesto en este trabajo, aplicado a tratamientos de lazo abierto CSII, con vista a una posible implementación en controladores de lazo cerrado, consiste en una restricción en la cantidad mínima de insulina a bordo disponible para evitar una hipoinsulinemia.

La idea consiste básicamente en reanudar antes de que finalice el ejercicio la infusión de insulina de forma tal que se disponga de un nivel mínimo de insulina disponible en el cuerpo. Para ello se realiza una en la estimación de $\mathrm{IOB}$ y asegurarse que el nivel estimado se mantenga por encima de un límite inferior prefijado por el usuario IOB_lim. El método propuesto puede ser implementado sencillamente vía software y aplicado a las tecnologías existentes.

La estimación de la insulina a bordo IOB es un recurso frecuente tanto en tratamientos a lazo abierto como a lazo cerrado para evitar riesgos de hipoglucemia debido al efecto de la acumulación de insulina en el cuerpo. La misma se puede utilizar para reducir bolos teniendo en cuenta el exceso por sobre valores basales [124, para el cálculo de super-bolos para contrarrestar eventos posprandiales agresivos [117, o el uso de restricciones a lazo cerrado 140,154. A diferencia de otro trabajos, en los cuales siempre se impone una restricción al nivel de IOB máximo, aquí se impone una cota al nivel mínimo de IOB. A fin de realiar la estimación de IOB, se utilizará el modelo discreto dado por la ec. 3.7 presentado en este Capítulo en la sección 3.1.

El pseudocódigo que describe al tratamiento propuesto se detalla en Algoritmo 3. El mismo corre en cada tiempo de muestreo de la bomba $\left(T_{s}\right)$. La propuesta trabaja de la siguiente manera: el sujeto antes de una $\mathrm{AF}$, realiza el anuncio de la misma (ex_flag) indicando el tiempo de comienzo de la actividad (ex_an) y su duración (ex_dur). Debido a la recomendación de reducir el suministro basal antes del comienzo de la AF se considera que el anuncio se produce por lo menos media hora de antelación. A partir de un tiempo de anticipo $\left(t_{-} a n\right)$, se interrumpe el suministro de basal de forma total. El algoritmo estima la evolución del perfil de \OB de los próximos 5 minutos (o el tiempo de ejecución de la bomba $T_{s}$ ) a partir de la insulina a inyectar $u_{f}$. El valor de $u_{f}$ se mantiene en cero $\left(K_{p 2}+0\right)$ en la medida que el nivel estimado de \OB se encuentre por encima del límite establecido de forma a-priori. En caso que el nivel sea menor o igual a $I O B \_l i m$, se reanuda un porcentaje $K_{p 1}$ del perfil de insulina basal del sujeto, $\operatorname{basal}\left(T_{s}\right)$. El límite $I O B \_l i m$ se define como un porcentaje del nivel de $\mathrm{IOB}$ basal

$$
I O B_{-} l i m=K_{p 1} \frac{2 \cdot \operatorname{basal}\left(T_{s}\right)}{K_{D I A}} .
$$

A su vez, se tiene en cuenta la diferencia entre el nivel estimado $I O B(K)$ y el límite impuesto para evitar transitorios excesivos por debajo del límite (IOB_dif). Finalmente, se guardan en un vector los valores de $u(k)$ obtenidos para los próximos 5 minutos y se 


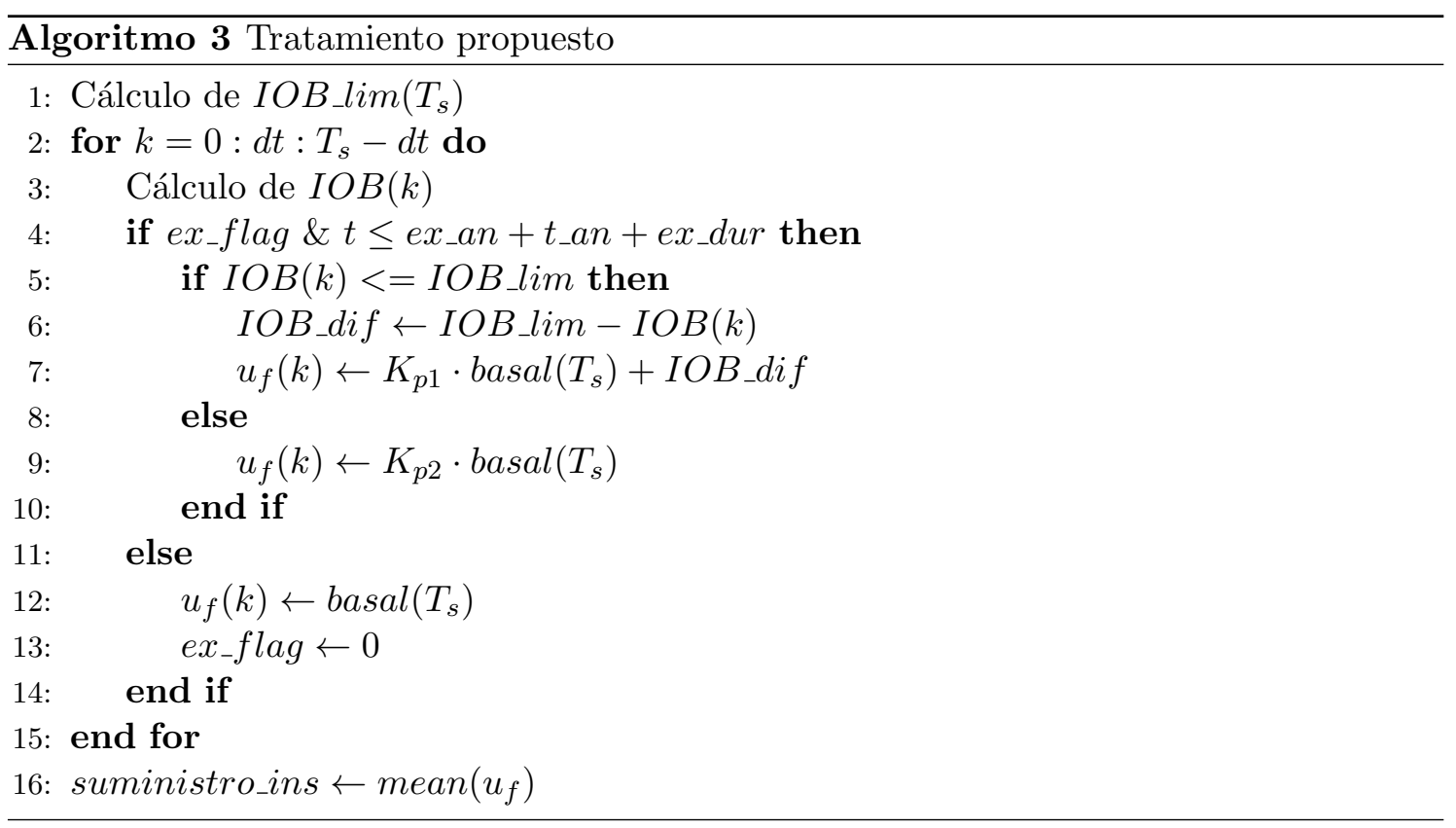

realiza un promedio del mismo para obtener la cantidad de insulina a ser suministrada por la bomba.

\subsubsection{Resultados}

A fin de evaluar el tratamiento propuesto en la sección 3.3.4 se realizó la modificación del modelo de la plataforma UVA/Padova de acuerdo a lo especificado en la sección 3.3.3. Los parámetros $a_{i}, \quad i=1,2, \ldots, 6$ se ajustaron de manera ad-hoc por medio de una simulación de Monte Carlo. Se decidió tomar los valores medios de la literatura (Cuadro 3.4) y considerar una variación del $30 \%$ de los mismos. Una vez ajustados los parámetros se logra modificar la influencia de las dinámicas agregadas mediante la entrada $u_{e x}$ (ec. (3.25)). La entrada que define la intensidad del ejercicio se varió con una distribución uniforme $u_{e x} \sim U[40 ; 100]$. A su vez, se aplicó variación a la SII Se definió una factor que afecta al parámetro $\alpha$ (ajustado al valor 3,29 de acuerdo a 151) de forma $K_{\alpha} \sim$ $U[0,75 ; 1,25]$.

Se plantea un escenario de ejercicio de 4 horas de duración $\left(e x_{-} d u r=240\right)$ de mediana intensidad ( $70 \%$ de $\mathrm{PVO}_{2}^{\max }$ ), lo que puede considerarse, por ejemplo, una actividad de caminata por la montaña o andar en bicicleta. El horario de anuncio del ejercicio es a las 3 horas de simulación $\left(e x_{\_} a n=180\right)$ y se optó por comenzar la suspensión de insulina 20 minutos antes de la $\mathrm{AF}\left(t_{-} a n=20\right)$. Se consideraron 3 casos de comparación: el caso I corresponde a la suspensión total del suministro de insulina basal $\left(K_{p 1}=0 ; K_{p 2}=0\right)$; el caso II corresponde a reducir el perfil de insulina basal a un $30 \%\left(K_{p 1}=0,3 ; K_{p 2}=0,3\right)$; y el caso III consiste en suspender el suministro de insulina basal al realizarse el anuncio pero reanudar el suministro de insulina cuando el nivel de $I O B(k)$ caiga por debajo del $30 \%$ del valor basal $\left(K_{p 1}=0,3 ; K_{p 2}=0\right)$.

En la Fig. 3.20 se presentan las simulaciones de Monte Carlo para cada caso, en las cuales se realiza un barrido de la entrada $u_{e x}$ y del parámetro $K_{\alpha}$ que afecta la SI. Se puede observar la GP media de los 10 adultos para cada simulación, la insulina suministrada, la estimación de IOB y la insulina en el espacio subcutáneo del modelo. Se delimitan los límites de hipoglucemia $(70 \mathrm{mg} / \mathrm{dl})$ e hiperglucemia $(180 \mathrm{mg} / \mathrm{dl})$ y el límite de IOB impuesto (IOB_lim).

$\mathrm{Al}$ comienzo del ejercicio, la concentración de GP disminuye debido al aumento de la SI y al aumento de utilización de glucosa de parte de los tejidos. El caso I permite evitar 

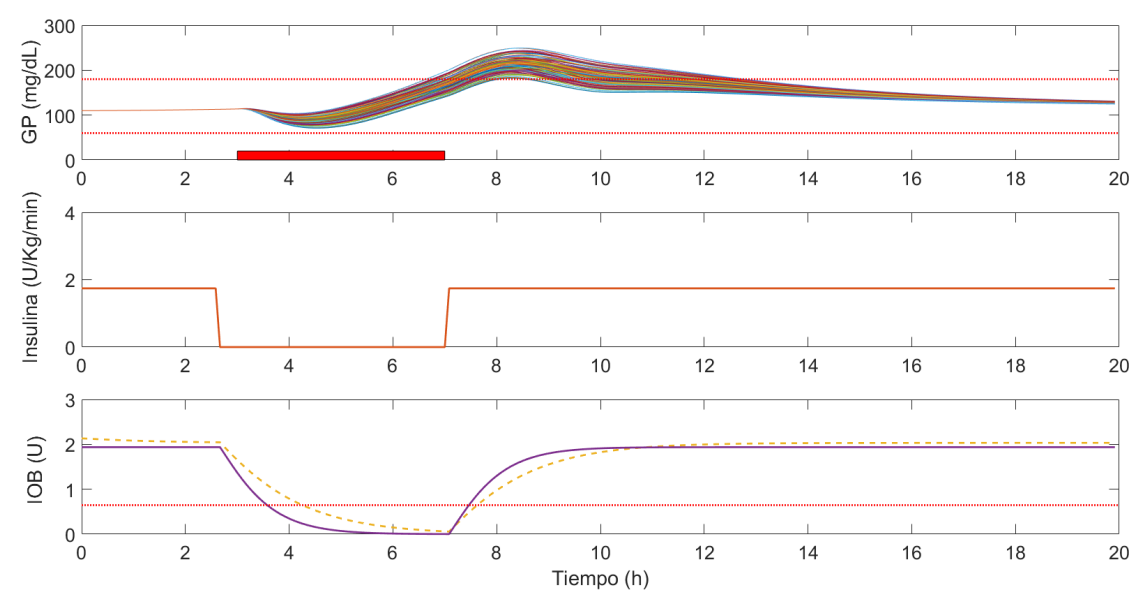

(a) Caso I
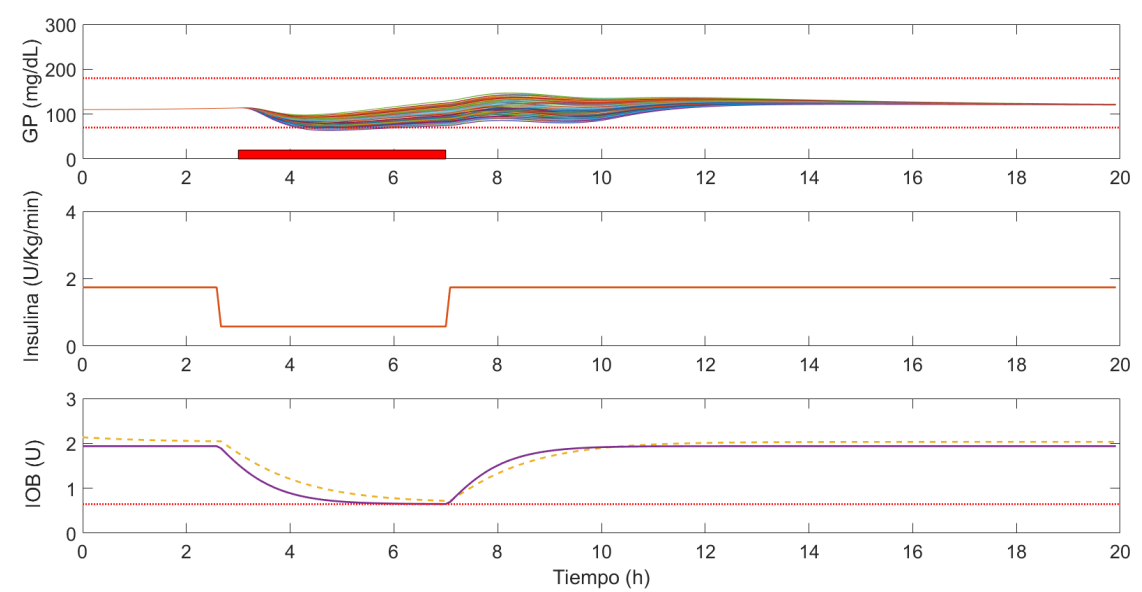

(b) Caso II
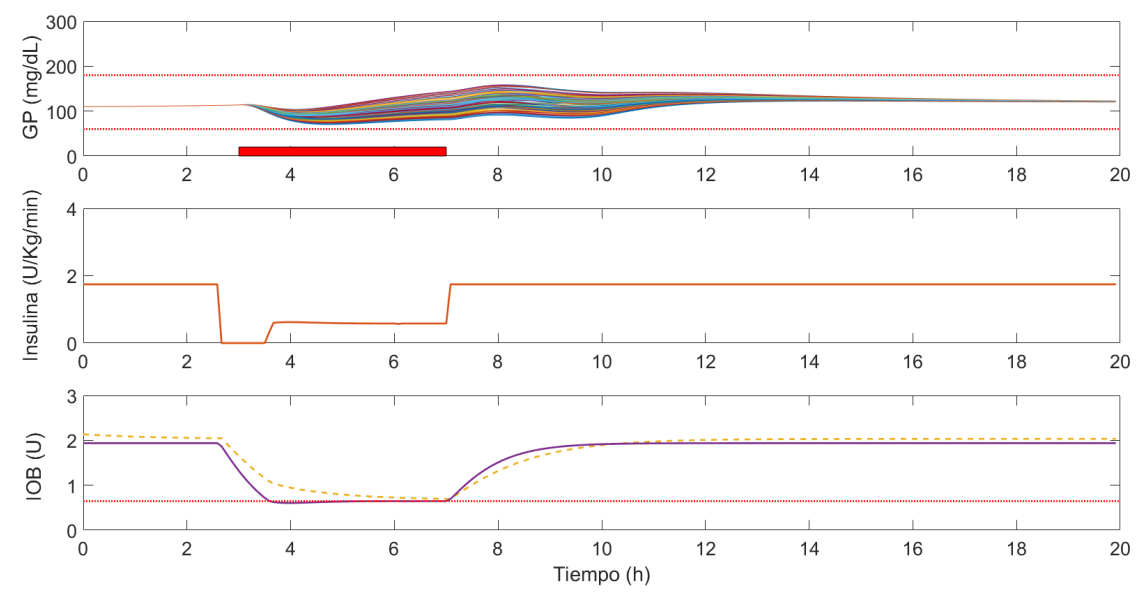

(c) Caso III

Figura 3.20: Simulación Monte Carlo con barrido en $K_{\alpha}$ y $u_{e x}$ para cada terapia propuesta. En cada figura se muestran la media de los 10 pacientes adultos para cada simulación: glucosa en plasma y límites de hipo- e hiperglucemia; insulina suministrada; insulina en el espacio subcutáneo (línea a trazos), IOB estimado (línea sólida) y el límite IOB_lim (línea punteada).

una posible hipoglucemia debido a que se disminuye la insulina en circulación, pero resulta agresiva la suspensión total de la misma y se observa un claro rebote glucémico, que lleva a la hiperglucemia. El caso II, que representa otra alternativa a la que acuden los sujetos conlleva a valores muy bajos de glucosa e incluso a un episodio hipoglucémico, lo cúal es 
totalmente indeseable. Por último, el caso III evita valores bajos de GP gracias a la gran disminución de insulina, pero a su vez evita la hiperglucemia fianl al asegurar un mínimo de insulina circulando en el cuerpo, manteniendo a los sujetos siempre en normoglucemia.

\subsection{Conclusiones}

En este capítulo se presentaron técnicas novedosas para el tratamiento a lazo abierto de la DMT1. Estas propuestas se basan en considerar la dinámica de la insulina subcutánea para regular las dosis de insulina durante dos situaciones particulares del control glucémico: el control posprandial y el control durante el ejercicio.

En el caso del control posprandial, se introduce un algoritmo que se basa en la implementación manual del super-bolo e introduce una metodología para su cálculo automático. El super-bolo automático basado en la IOB permite un mejor rendimiento durante el control posprandial frente a comidas con un alto índice glucémico y en particular, con alto contenido de carbohidratos en comparación con el tratamiento estándar. Se reduce la excursión glucémica, tal y como preveía la justificación teórica, siendo capaz de prevenir eventos hipoglucémicos en periodos posprandiales tardíos.

Por otro lado, la actividad física es un componente importante en el manejo de la diabetes mellitus tipo 1. Las medidas difundidas para el control de la glucosa durante el ejercicio, como reducir un porcentaje el suministro de insulina basal, pueden resultar ser poco efectivas y exponer al sujeto a una posible hipoglucemia. A su vez, prácticas más agresivas como reducir drásticamente la insulina que circula por el cuerpo, en particular durante ejercicios de larga duración y mediana intensidad, pueden provocar un rebote en la concentración de glucosa en plasma luego de finalizada la actividad, conduciendo a la hiperglucemia. Se propone un tratamiento seguro para mitigar las hiperglucémias durante o después del ejercicio. Para esto, se propone además una modificación al modelo ampliamente difundido UVA/Padova con el objetivo de representar los efectos del ejercicio en la dinámica del sistema glucosa-insulina, en particular el efecto rebote en la glucosa en plasma. 
$\sqrt{c a n+\infty} 4$

\section{Estrategias de control a Lazo-Cerrado vía limitación de IOB}

En este capítulo se presentan dos enfoques para el control de glucosa de lazo cerrado: uno en configuración lazo híbrido o semiautomático, donde hay acción a lazo abierto por parte del sujeto (acción feedfoward para la compensación de comidas); y otro de lazo cerrado puro o automático, en donde sólo se cuenta con la acción del controlador para la administración de insulina.

A la hora de diseñar sistemas de Páncreas Artificial, se deben recordar las limitaciones de control que afectan a su rendimiento, en particular las restricciones de la ruta subcutánea. Esto incluye:

- Grandes perturbaciones (comidas, ejercicio, entre otras).

- Retardos, siendo la respuesta del sujeto a la insulina es más lenta que a la ingesta de alimentos.

- No hay acción negativa (la insulina se puede administrar pero no extraer).

- Gran variación inter- e intra-paciente.

Estas restricciones, junto con las limitaciones tecnológicas, no permiten todavía un control glucémico totalmente automático. Hoy en día la gran mayoría de los ensayos clínicos evalúan estrategias de control híbridas, es decir, una combinación de un bolo de insulina (que se calcula a partir de la información de la comida que se va a ingerir) y un algoritmo de control que administra insulina durante el período posprandial tardío $155-158$. Sin embargo, lograr un control completo de lazo cerrado sigue siendo el objetivo principal. También se han evaluado algoritmos completamente automáticos en ensayos ambulatorios, pero principalmente en el período nocturno, cuando no hay perturbaciones presentes en el transcurso de la noche. Sólo se han realizado unos pocos ensayos de lazo cerrado tanto de día como de noche 159,160 .

Como se mencionó en el Capítulo 1, en el tratamiento de la diabetes la hipoglucemia es el riesgo que más preocupa a los sujetos. La hipoglucemia suele ser el resultado de una sobre-estimación de la dosis de insulina por parte del controlador (el retraso en la respuesta del sistema incita a la acumulación de insulina). El uso de restricciones en IOB para prevenir esta hipoglucemia inducida por la insulina ha demostrado que mejora el control de la glucemia tanto in-silico como in-vivo [140]. Por ejemplo, estas restricciones pueden ser abordadas por una estrategia de control MPC [140]. Con los controladores MPC la restricción se tiene en cuenta explícitamente durante el diseño del controlador. Por el contrario, existen otras técnicas que permiten diseñar el controlador principal por separado sin incluir las limitaciones de IOB y añadir la capa de seguridad que tiene en 
cuenta la restricción deseada (diseño de dos pasos), como la capa Safety Auxiliary Feedback Element (SAFE) presentada por el grupo de trabajo y colaboradores [141]. De esta manera, los controladores potencialmente más simples que no serían capaces de manejar las restricciones de IOB pueden incorporarlas como un mecanismo de seguridad.

A partir de la presentación de la capa SAFE surgieron diferentes horizontes de aplicación. Esta capa consiste en imponer sobre un controlador principal una restricción sobre la insulina activa. Uno de las primeras implementaciones se presenta en la sección 4.2, y da lugar a un funcionamiento del controlador denominado como Time Enable. Aquí se utiliza a la capa SAFE como protección ante la sobreactuación en la prueba de controladores híbridos que pueden no estar bien sintonizados. Esta propuesta fue presentada en el congreso internacional ATTD 2017 [161] y luego publicado en una revista científica [154].

Por otro lado, en un trabajo colaborativo entre el ITBA y la UNLP, se desarrolló el algoritmo Automatic Regultaion of Glucose (ARG), un controlador basado en una estructura Linear Quadratic Gaussian (LQG) al cual se le agrega la capa SAFE como prevención de hipoglucemias [159]. Este algoritmo de lazo cerrado cuenta con la particularidad de no suministrar bolos pre-prandiales. A su vez, fue evaluado en los primeros ensayos clínicos de un sistema de PA en el país y en Latinoamérica. Estos ensayos contaron con dos fases: la fase 1 consistió en un entrenamiento para el equipo de trabajo, donde se ensayó un algoritmo de la Universidad de Virginia [158]; y la fase 2 donde se ensayó el algoritmo ARG. Este último fue presentado en una revista de alto impacto en [20].

\subsection{Algoritmo SAFE}

La capa de seguridad SAFE (Safety Auxiliary Feedback Element) está basada en la técnica de acondicionamiento de señales por modos deslizantes -Sliding-Mode Reference Conditioning (SMRC)-, método desarrollado por los directores y colegas y explicada en mayor detalle en [162]. Esta técnica, desarrollada en general para el control de sistemas dinámicos con restricciones, fue aplicada por primera vez al problema de la[DMT1en [141]. Este algoritmo funciona como una capa de seguridad añadiendo una restricción en la IOB alrededor de cualquier controlador principal y ha demostrado reducir el número y la gravedad de los eventos hipoglucémicos [94]. Esta estrategia ha sido validada con éxito en ensayos clínicos como parte de un controlador principal en España y Argentina [20, 163]. De éste último ensayo clínico, en el cual se participó, se dará más información en la sección 4.3.6 de este Capítulo.

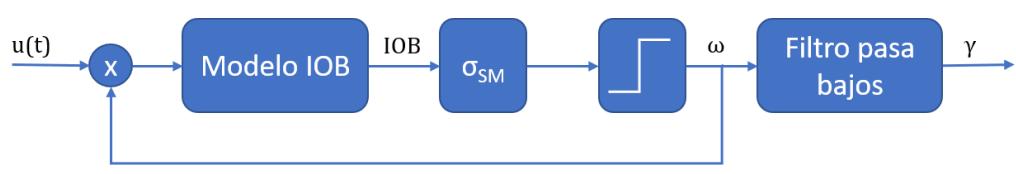

Figura 4.1: Diagrama en bloques de la capa de seguridad SAFE.

El principal objetivo de la capa SAFE es modular la ganancia del controlador para evitar que la insulina activa o IOB supere un determinado umbral $\overline{\mathrm{IOB}}$, reduciendo así el riesgo de hipoglucemias en los períodos posprandiales tardíos. En la Figura 4.1 se puede visualizar el diagrama de bloques de la capa SAFE. Como en todo control por modo deslizante, el elemento clave es el bloque conmutado, cuya lógica de conmutación es:

$$
w(t)= \begin{cases}1 & \text { si } \sigma_{\mathrm{SM}}(t)>0 \\ 0 & \text { caso contrario }\end{cases}
$$

siendo la función de deslizamiento $\sigma_{\mathrm{SM}}(t)$ en este caso simplemente la diferencia entre la insulina activa actual y su correspondiente límite:

$$
\sigma_{\mathrm{SM}}(t)=\overline{\mathrm{IOB}}(t)-\mathrm{IOB}(t) .
$$


Gran parte del diseño de esta capa se encuentra en establecer el límite $\overline{\mathrm{IOB}}(t)$. Este límite puede ser constante en el tiempo, constante a tramos o variante en el tiempo. En este Capítulo se verán diferentes formas de establecerlo.

Debido a que la IOB no puede ser medida en tiempo real, la misma debe ser estimada. Para ello, se consideró el modelo presentado en la sección 3.1 ec. (3.5), el cual tiene la ventaja de que puede ser personalizado basándose sólo en un único parámetro clínico [120].

$\mathrm{Al}$ alcanzarse el límite $\overline{\mathrm{IOB}}(t)$ se establece un régimen deslizante sobre la superficie $\sigma_{\mathrm{SM}}(t)=0$. Durante este modo, a partir de (4.1), la señal $w(t)$ conmuta a muy alta frecuencia entre 0 y 1 de manera de cumplir con la restricción impuesta y forzar al sistema (3.5) a permanecer dentro del conjunto invariante

$$
\Sigma=\left\{x(t) \mid \sigma_{\mathrm{SM}}(t) \geq 0\right\}
$$

donde $x(t) \in \mathbb{R}^{2}$ son los estados de (3.5). La señal conmutada $w(t)$ es suavizada por un filtro de primer orden (o bien promediada entre intervalos de infusión), dando lugar a $\gamma(t)$, que es el factor por el que se atenúa la señal comandada a la bomba.

Es fácil demostrar que la derivada de la función de conmutación $\sigma_{\mathrm{SM}}(t)$ depende de la acción $u_{w}(t)$ para el modelo dado por (3.5), y por tanto de la acción discontinua $w(t)$, lo que es una condición necesaria para el establecimiento del modo deslizante, conocida como Condición de Transversalidad [164]. En efectoel modelo (3.5) se puede reescribir de la siguiente forma:

$$
\begin{aligned}
\dot{x}(t)= & f(x)+g(x) u_{w}(t)=\cdots \\
& \cdots=\left[\begin{array}{c}
-K_{\mathrm{DIA}} I_{s c 1}(t) \\
K_{\mathrm{DIA}}\left[I_{s c 1}(t)-I_{s c 2}(t)\right]
\end{array}\right]+\left[\begin{array}{l}
1 \\
0
\end{array}\right] u_{w}(t)
\end{aligned}
$$

donde $f(x): \mathbb{R}^{2} \rightarrow \mathbb{R}^{2}$ y $g(x): \mathbb{R}^{1} \rightarrow \mathbb{R}^{2}$ son campos vectoriales. La condición de transversalidad consiste en $L_{g} \sigma_{\mathrm{SM}}(t)=\frac{\partial \sigma_{\mathrm{SM}}(t)}{\partial x}(t) g(x) \neq 0$. Entonces, en este caso se obtiene:

$$
L_{g} \sigma_{\mathrm{SM}}(t)=\left[\begin{array}{ll}
-1 & -1
\end{array}\right]\left[\begin{array}{l}
1 \\
0
\end{array}\right]=-1 \neq 0
$$

verificándose que siempre se cumple la condición de tranversalidad.

\subsection{Lazo híbrido: Algoritmo Time Enable}

El algoritmo Time Enable se define para ser utilizado en configuraciones híbridas. El lazo SAFE propuesto anteriormente [94,141] donde se utiliza una restricción IOB constante se reinterpreta para ser usado en ensayos clínicos. Se busca proporcionar un criterio para establecer la restricción IOB y, a partir de ese punto, disminuir de forma segura la acción de lazo-abierto para dar paso al controlador de lazo-cerrado. También funciona como un mecanismo de seguridad contra los controladores mal sintonizados, reduciendo la gravedad y la duración de los posibles eventos hipoglucémicos. En esta configuración de lazo-híbrido (ver Fig.4.2), el bolo administrado por tratamiento de lazo-abierto está fuera del lazo de la capa SAFE y por lo tanto no se ve afectado por el factor de escala $\gamma$, independientemente de si viola la restricción o no.

En el algoritmo Time Enable, la capa SAFE habilita gradualmente en el tiempo la acción de lazo-cerrado después de un bolo de comida. Más específicamente, después de un periodo posprandial temprano en el que una comida ha sido exclusivamente compensada por la acción de lazo-abierto. La habilitación por tiempo permite al controlador reanudar la administración de insulina de forma gradual y automática (atenuando su ganancia según sea necesario de acuerdo con la restricción impuesta en la IOB) hasta que el lazo-cerrado 


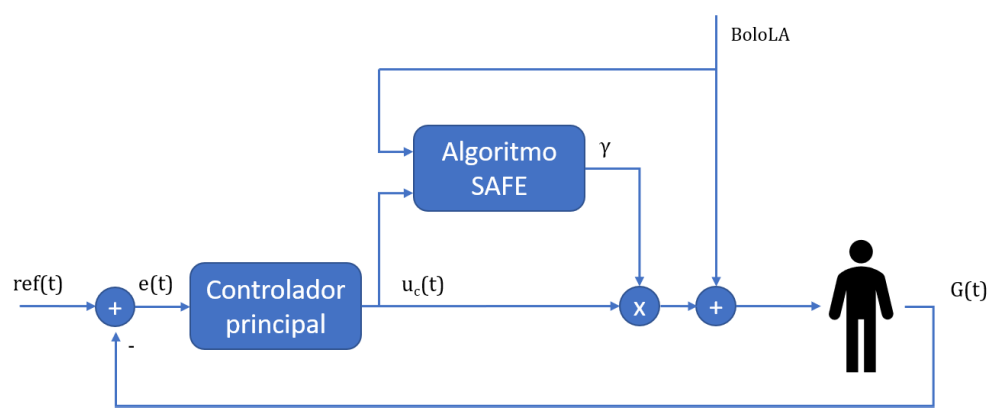

Figura 4.2: Diagrama de bloques que representa un lazo híbrido de control de glucosa junto a la capa SAFE.

esté completamente habilitado $(\gamma=1)$. La capa SAFE se plantea como una herramienta para proteger al sujeto de una hipoglucemia grave durante ensayos clínicos. Luego, si es necesario, permite la puesta a punto segura del controlador principal de glucosa, liberando eventualmente al controlador de la necesidad de la protección de SAFE.

A partir del funcionamiento de la capa SAFE, se debe esperar que ocurra lo siguiente después de una ingesta de comida: primero, cuando se administra el bolo de insulina, el $\overline{I O B}$ es superado inevitablemente, dando como resultado $\gamma=0$. Como resultado, la salida del controlador debería ser 0 , por lo que no se administra insulina adicional y el sistema de control funciona en lazo-abierto. El nivel de IOB disminuye naturalmente a medida que la insulina es absorbida de acuerdo con el DIA del sujeto. Después de un tiempo $t_{L A}$, la IOB alcanza el nivel $\overline{I O B}$ y la capa SAFE comienza a cambiar internamente entre $\omega=0$ y $\omega=1$. La salida $\gamma$ (resultado de la media de un vector de $\omega$ a lo largo de cada tiempo de muestreo) será estrictamente un valor entre 0 y 1 . Entonces, la capa SAFE permite al controlador entregar insulina escalada en $\gamma$. La duración de este proceso se llamará $t_{S A F E}$. Cuando finalmente la respuesta del controlador ya no provoca que se supere el límite, la capa SAFE se desactiva. Esto inicia el periodo de tiempo llamado $t_{L C}$. De esta manera, podemos identificar tres fases distintivas: lazo-abierto, transición y lazo-cerrado.

$\mathrm{El}$ algoritmo SAFE puede funcionar alrededor de cualquier controlador principal de glucosa. Para ilustrar el funcionamiento del algoritmo propuesto, se implementa un controlador principal denominado PDBasal. Este se basa en el clásico algoritmo de control PID 165 en donde el término integral se sustituye por la insulina basal de la terapia de lazo-abierto. De este forma, se elimina una posible sobreactuación debido al término integral del controlador y el efecto windup. La salida del controlador PDBasal se define de la siguiente forma:

$$
u_{c}(t)=k_{p}\left[e(t)+\tau_{d} \frac{d e(t)}{d t}\right]+u_{\text {basal }}(t)
$$

donde la ganancia proporcional $k_{p}$ se define como

$$
k_{p}=\frac{60}{\tau_{d}} \frac{I_{T D D}}{1500}
$$

siendo $I_{T D D}$ la insulina total diaria [166], y $\tau_{d}(t)$ posee un valor nominal de $90 \mathrm{~min}$ durante el día y 60min durante la noche 165.

Debido a que se presenta una configuración híbrida (Fig. 4.2), el total de insulina suministrada es la suma de $u_{c}(t)$ y el bolo de lazo-abierto ("BoloLA") calculado como:

$$
\text { BoloLA }=k_{L A} \cdot \frac{C H O}{C R}
$$

donde CR es la relación insulina/carbohidratos del paciente, $C H O$ es la cantidad a ingerir de carbohidratos y $k_{L A}$ es un factor que escala el tamaño del bolo de insulina. 


\subsubsection{Ejemplo ilustrativo}

La figura 4.3 ilustra el funcionamiento del algoritmo propuesto (líneas sólidas) en el sujeto virtual Adulto\#1 del simulador UVA/Padova ante una comida de 60g de carbohidratos. El controlador principal consiste en el PDBasal (ecuaciones (4.6) y (4.7)) más el bolo de insulina de la comida 4.8 escalado en $k_{L A}=0,7$. Para fines comparativos, la respuesta del controlador sin la protección de la capa SAFE se muestra en línea discontinua. El área gris corresponde al rango de glucosa objetivo $(70-180 \mathrm{mg} / \mathrm{dl})$. La comida comienza en el tiempo $t=10 \mathrm{~min}$. En ese momento se administra el bolo de insulina, por lo que el nivel de IOB aumenta hasta su valor máximo casi instantáneamente (para la dinámica del sistema el bolo se comporta como un delta de Kronecker). Al superarse $\overline{I O B}$, la ganancia adaptativa $\gamma$ cambia a 0 y permanece así durante todo $t_{L A}$. Durante este tiempo el controlador no administra insulina, suspendiéndose a su vez el suministro basal del lazo-abierto. Este comportamiento es similar a un super-bolo 14, 117]. Sin embargo, se debe tener en cuenta que la capa SAFE también puede configurarse para no suspender la administración de insulina basal durante este período. Luego, puede observarse que en $t=2,2 h s$ el nivel de IOB alcanza $\overline{I O B}$. Cuando esto sucede, comienza una conmutación rápida entre $0 \mathrm{y}$ 1 en $\omega$, permitiendo así que el controlador administre la insulina de forma gradual sin superar la restricción $(0<\gamma<1)$. En aproximadamente $t=5,8 h s$, el $t_{S A F E}$ termina y el controlador comienza a actuar libremente $(\gamma=1)$. Por el contrario, cuando no se utiliza la capa SAFE, el controlador PDBasal entrega una gran cantidad de insulina en el instante posterior al bolo de lazo-abierto. Como resultado, se observan valores glucémicos posprandiales más bajos, así como una excursión de glucosa más grande y un transitorio mayor. Esto se debe a la sobre-estimación de la dosis de insulina debido a la superposición de la acción del controlador con el bolo de comida escalado. El doble pico en la concentración de glucosa presente en este sujeto refuerza la sobredosis de insulina.
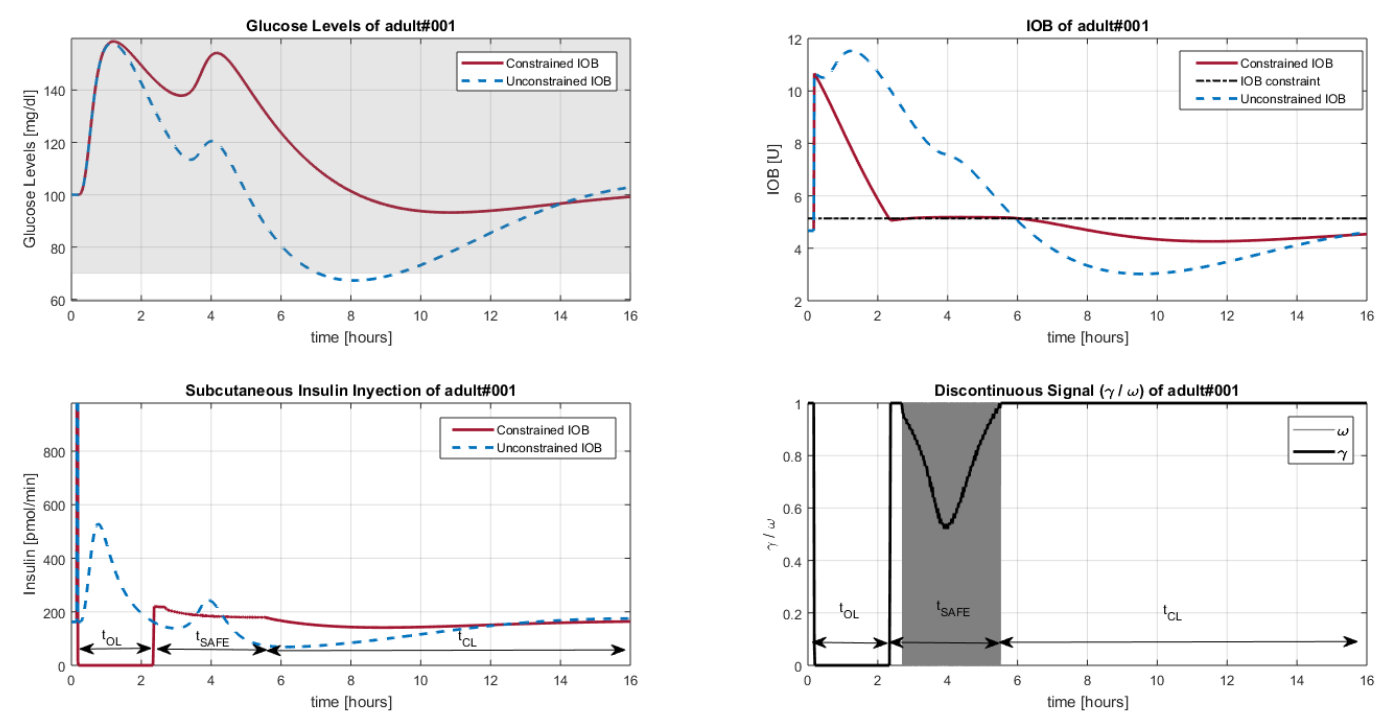

Figura 4.3: Señales principales del modo de operación Time Enable sobre el Adulto \#1 del simulador UVA/Padova ante una comida de $60 \mathrm{~g}$ de $\mathrm{CHO}$.

\subsubsection{Análisis in-silico}

En este apartado se muestran simulaciones realizadas sobre la cohorte de 30 sujetos virtuales (10 adultos, 10 adolescentes y 10 niños) para mostrar los efectos de la variabilidad inter-paciente. Se consideraron dos enfoques para el análisis del método propuesto: un 
barrido de valores del límite $\overline{I O B}$ y otro de la ganancia $k_{P}$ del controlador principal.

\section{Barrido de $\overline{I O B}$ : estableciendo una restricción en IOB}

El escenario de simulación que se considera aquí consiste en una comida única de $80 \mathrm{~g}$ de $\mathrm{CHO}$ puro en un periodo de observación de $16 \mathrm{hs}$. Se realiza un barrido $\overline{I O B}$ en un intervalo dado por el perfil de absorción de un bolo. En el extremo inferior del intervalo, $\overline{I O B}_{\min }$ se establece en correspondencia con el nivel IOB basal de cada sujeto. En el otro extremo, $\overline{I O B}_{\max }$ se establece como el valor máximo de IOB obtenido cuando se administra un bolo de lazo-abierto para una comida específica (este valor dependerá de la cantidad de $\mathrm{CHO}$ y $k_{L A}$ ). Estos dos valores se muestran en la figura 4.4 . Se toman seis valores intermedios de $\overline{I O B}$ entre los extremos definidos.

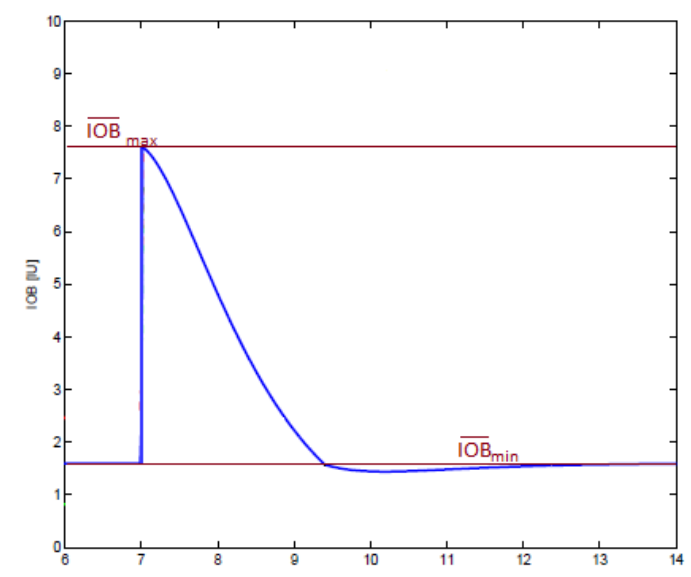

Figura 4.4: Los límites $\overline{I O B}_{\text {min }}$ y $\overline{I O B}_{\max }$ ilustrados de acuerdo al perfil de IOB en el tiempo de un bolo de $6 \mathrm{U}$ de insulina.

El cuadro 4.1 muestra los resultados obtenidos al realizar el barrido de $\overline{I O B}$ para $k_{L A}=1$. Se muestra para cada simulación el valor medio de $t_{L A}, t_{L C}, t_{S A F E}$, el tiempo en hipoglucemia $\left(t_{\text {hypo }}\right)$, el tiempo en normoglucemia $\left(t_{\text {normo }}\right)$ y el tiempo en hiperglucemia $\left(t_{\text {hyper }}\right)$. A partir de estos resultados puede observarse que los valores de $\overline{I O B}^{\prime} s$ menos restrictivos resultan en una mayor cantidad de eventos hipoglucémicos. Esto se debe a que cuanto mayor sea el nivel $\overline{I O B}$, más larga será la superposición de las terapias de lazo-abierto y -cerrado, las cuales están sintonizadas para funcionar por separado. Por otro lado, cuando la capa SAFE resulta demasiada restrictiva, la cantidad de insulina administrada es menor que la terapia de lazo-abierto, ya que el controlador sólo puede indicar valores menores o iguales a la insulina basal, lo que resulta en un aumento de la hiperglucemia. No obstante, la elección de una restricción de IOB conservadora podría ser una opción apropiada. Por ejemplo, se puede ver en estos resultados que si se utiliza el bolo de insulina de lazo-abierto en su totalidad $\left(k_{L A}=1\right)$, el $\overline{I O B}$ debería ajustarse cercano a $\overline{I O B}_{\text {min }}$ para obtener el mayor porcentaje de tiempo en la normoglucemia (ya que el tratamiento a lazo-abierto resulta óptimo en condiciones ideales).

Sin embargo, es común en los ensayos clínicos de algoritmos de control híbridos administrar un bolo de comida más pequeño que en la terapia de lazo-abierto. Por ejemplo, el uso de $k_{L A}=0,5$ ha sido probado clínicamente [167. Esta elección se toma para evitar el apilamiento excesivo de insulina, sin reducir la acción de lazo-cerrado, ya que la consecuencia de reducirla es que el control sea menos robusto a las variaciones en la composición de la comida y en la sensibilidad a la insulina, entre otros factores. Para ilustrar este caso, se proponen dos escenarios para el barrido de $\overline{I O B}$ utilizando valores de $k_{L A}=0,7 \mathrm{y}$ 
$k_{L A}=0,5$. Los resultados de estas simulaciones se muestran en las tablas 4.2 y 4.3 . Se puede observar que cuando $k_{L A}=0,7$ (tabla 4.2 ), el ajuste de $\overline{I O B}$ a un valor intermedio entre $\overline{I O B}_{\min }$ y $\overline{I O B}_{\max }\left(\overline{I O B}_{5}\right)$ maximiza el tiempo en el rango normoglucémico. Además, usando $\overline{I O B}_{5}$ resulta en $t_{L C}=14,37 \mathrm{hs}$, lo que significa que el controlador regula la glucemia libremente $89,8 \%$ del tiempo total de simulación. En el caso de reducir aún más el bolo de lazo-abierto, puede observarse en la tabla 4.3 que para $k_{L A}=0,5$ el rango normoglucémico se maximiza cuando $\overline{I O B}$ se ajusta a $\overline{I O B}_{7}$. En este caso, $t_{L C}=14,48 \mathrm{hs}$, representando un 90,5\% del tiempo total de simulación.

En conclusión, a medida que el bolo de lazo-abierto se reduce para dar paso al control de lazo-cerrado, se debe aumentar el límite $\overline{I O B}$ (respecto a $\overline{I O B}_{\text {min }}$ ) para lograr la mayor cantidad de tiempo en la normoglucemia manteniendo un $t_{L C}$ razonable.

Tabla 4.1: Barrido de $\overline{I O B}$ para los 30 sujetos virtuales utilizando $k_{L A}=1$.

\begin{tabular}{lllllll}
\hline$\overline{I O B}[U]$ & $t_{L A}$ medio $[\mathrm{hs}]$ & $t_{S A F E}$ medio $[\mathrm{hs}]$ & $t_{L C}$ medio [hs] & $t_{\text {hypo }}$ medio [\%] & $t_{\text {normo }}$ medio [\%] & $t_{\text {hyper }}$ medio [\%] \\
\hline$\overline{I O B}_{\text {min }}$ & 2,53 & 6,05 & 7,44 & 0 & 91,69 & 8,31 \\
$\overline{I O B}_{2}$ & 1,96 & 0,78 & 13,28 & 2,47 & 90,97 & 6,56 \\
$\overline{I O B}_{3}$ & 1,56 & 0,28 & 14,18 & 4,35 & 89,50 & 6,15 \\
$\overline{I O B}_{4}$ & 1,23 & 0,22 & 14,56 & 5,57 & 88,82 & 5,61 \\
$\overline{I O B}_{5}$ & 0,95 & 0,27 & 14,80 & 7,00 & 87,83 & 5,16 \\
$\overline{I O B}_{6}$ & 0,67 & 0,38 & 14,96 & 9,67 & 85,71 & 4,62 \\
$\overline{I O B}_{7}$ & 0,39 & 0,50 & 15,13 & 11,79 & 84,03 & 4,18 \\
$\overline{I O B}$ & 0 & 0,27 & 15,74 & 15,05 & 81,10 & 3,85 \\
\hline
\end{tabular}

Tabla 4.2: Barrido de $\overline{I O B}$ para los 30 sujetos virtuales utilizando $k_{L A}=0,7$.

\begin{tabular}{lllllll}
\hline$\overline{I O B}[U]$ & $t_{L A}[\mathrm{hs}]$ medio & $t_{S A F E}[\mathrm{hs}]$ medio & $t_{L C}[\mathrm{hs}]$ medio & $t_{\text {hypo }}[\%]$ medio & $t_{\text {normo }}[\%]$ medio & $t_{\text {hyper }}[\%]$ medio \\
\hline$\overline{I O B}_{\text {min }}$ & 2,17 & 7,63 & 6,22 & 0 & 83,13 & 16,87 \\
$\overline{I O B}_{2}$ & 1,73 & 1,92 & 12,37 & 0,84 & 85,96 & 13,20 \\
$\overline{I O B}_{3}$ & 1,39 & 1,14 & 13,49 & 2,64 & 86,06 & 11,30 \\
$\overline{I O B}_{4}$ & 1,11 & 0,90 & 14,01 & 3,88 & 86,64 & 9,48 \\
$\overline{I O B}_{5}$ & 0,85 & 0,80 & 14,37 & 4,86 & 87,10 & 8,05 \\
$\overline{I O B}_{6}$ & 0,60 & 0,81 & 14,60 & 5,81 & 87,07 & 7,12 \\
$\overline{I O B}_{7}$ & 0,34 & 0,81 & 14,87 & 6,89 & 86,77 & 6,34 \\
$\overline{I O B}_{\max }$ & 0 & 0,56 & 15,46 & 9,11 & 85,19 & 5,70 \\
\hline
\end{tabular}

Tabla 4.3: Barrido de $\overline{I O B}$ para los 30 sujetos virtuales utilizando $k_{L A}=0,5$.

\begin{tabular}{lllllll}
\hline$\overline{I O B}[U]$ & $t_{L A}[\mathrm{hs}]$ medio & $t_{S A F E}[\mathrm{hs}]$ medio & $t_{L C}[\mathrm{hs}]$ medio & $t_{\text {hypo }}[\%]$ medio & $t_{\text {normo }}[\%]$ medio & $t_{\text {hyper }}[\%]$ medio \\
\hline$\overline{I O B}_{\min }$ & 1,84 & 8,81 & 5,36 & 0 & 74,61 & 25,39 \\
$\overline{I O B}_{2}$ & 1,50 & 3,38 & 11,14 & 0 & 81,38 & 18,62 \\
$\overline{I O B}_{3}$ & 1,22 & 2,40 & 12,40 & 1,38 & 83,35 & 15,27 \\
$\overline{I O B}_{4}$ & 0,97 & 1,73 & 13,31 & 2,73 & 83,72 & 13,55 \\
$\overline{I O B}_{5}$ & 0,75 & 1,53 & 13,74 & 3,22 & 84,63 & 12,16 \\
$\overline{I O B}_{6}$ & 0,52 & 1,39 & 14,10 & 4,09 & 85,39 & 10,51 \\
$\overline{I O B}_{7}$ & 0,29 & 1,25 & 14,48 & 4,92 & 86,10 & 8,98 \\
$\overline{I O B}_{\text {max }}$ & 0 & 0,94 & 15,08 & 5,66 & 85,61 & 7,73 \\
\hline
\end{tabular}

\section{Barrido de $k_{P}$ : protección ante controladores mal sintonizados}

El escenario de simulación que se muestra aquí consiste nuevamente de una comida única de $80 \mathrm{~g}$ de $\mathrm{CHO}$ puro en un período de observación de $16 \mathrm{hs}$ sobre la cohorte de 30 sujetos. Ahora se realiza un barrido de $k_{p}$ para simular las consecuencias de un controlador sintonizado inadecuadamente. La ganancia del controlador se fija en $50 \%, 75 \%, 100 \%$, $125 \%, 150 \%, 175 \%$ y $200 \%$ de su valor nominal. El bolo se ajusta con $k_{L A}=0,7$ y el límite $\overline{I O B}$ se fija en $\overline{I O B}_{5}$, a partir de los resultados obtenidos en la subsección anterior. La simulación se repite para el mismo escenario pero utilizando el controlador PDBasal en configuración híbrida sin (limitación de IOB) el Time Enable. 
La tabla 4.4 muestra los resultados obtenidos utilizando el barrido de $k_{p}$. Se puede observar la media de $t_{L A}, t_{L C}, t_{S A F E}$, el tiempo en hipoglucemia $\left(t_{\text {hypo }}\right)$, el tiempo en normoglucemia $\left(t_{\text {normo }}\right)$ y el tiempo en hiperglucemia $\left(t_{\text {hyper }}\right)$ obtenidos para cada simulación. Cabe señalar que un cambio en $k_{p}$ no afecta a $t_{L A}$, pero sí tiene un impacto en $t_{S A F E}$. Para mayores ganancias del controlador principal, el $t_{S A F E}$ será más largo, ya que un controlador más agresivo estará más tiempo restringido. Las seis columnas de la derecha de esta tabla evidencian que el algoritmo Time Enable permite que la excursión de la glucosa en sangre sea menos sensible a los cambios de ganancia del controlador. Por lo tanto, se puede afirmar que la utilización de la capa de seguridad permite probar un controlador cuya sintonización no se encuentre ajustada, sin exponer al sujeto a episodios hipoglucémicos graves.

Tabla 4.4: Barrido de $k_{p}$ sobre los 30 sujetos virtuales. PDBasal en lazo-híbrido utilizando $k_{L A}=0,7$ con (columnas blancas) y sin (columnas sombreadas) el algoritmo Time Enable.

\begin{tabular}{llllllllll}
\hline \multirow{2}{*}{$k_{p}$} & \multirow{2}{*}{$t_{L A}$ medio } & \multirow{2}{*}{$t_{S A F E}$ medio } & \multirow{2}{*}{$t_{L C}$ medio } & \multicolumn{2}{c}{$t_{\text {hypo }}$ medio } & \multicolumn{3}{c}{$t_{\text {normo }}$ medio } & \multicolumn{2}{c}{$t_{\text {hyper }}$ medio } \\
con TE & sin TE & con TE & sin TE & con TE & sin TE \\
\hline $50 \%$ & 0,85 & 0,36 & 14,81 & 3,93 & 11,12 & 87,71 & 83,04 & 8,36 & 5,84 \\
$75 \%$ & 0,85 & 0,60 & 14,57 & 4,46 & 12,23 & 87,41 & 82,48 & 8,12 & 5,29 \\
$100 \%$ & 0,85 & 0,80 & 14,37 & 4,86 & 14,02 & 87,10 & 81,02 & 8,05 & 4,97 \\
$125 \%$ & 0,85 & 0,94 & 14,23 & 6,03 & 15,84 & 86,00 & 79,46 & 7,97 & 4,70 \\
$150 \%$ & 0,85 & 1,07 & 14,10 & 7,13 & 19,28 & 84,99 & 76,23 & 7,87 & 4,50 \\
$175 \%$ & 0,85 & 1,15 & 14,02 & 8,28 & 22,91 & 83,88 & 72,77 & 7,84 & 4,32 \\
$200 \%$ & 0,85 & 1,22 & 13,95 & 9,11 & 25,40 & 83.08 & 70,44 & 7,81 & 4,15 \\
\hline
\end{tabular}

Otro método que parte del desarrollo de restricciones sobre la IOB es el desarrollado por Emilia Fushimi y presentado también en [154], llamado Amplitude Enable. Este método se centra en ensayos clínicos de lazo-cerrado. Este modo de funcionamiento fue diseñado para asegurar que la acción del controlador no exceda en una medida determinada la infusión de insulina de la terapia tradicional. En este caso, la limitación de la IOB se basa en el perfil de IOB variante en el tiempo que resultaría de un tratamiento de lazo-abierto para las comidas consideradas para el ensayo.

\subsection{Lazo cerrado: Algoritmo ARG}

El diseño y prueba del algoritmo ARG se llevó a cabo en colaboración entre los grupos de trabajo del ITBA y la Universidad Nacional de La Plata. Este fue un proceso de algunos años y de diferentes etapas. En primer lugar, el Dr. Patricio Colmegna, bajo la dirección del Dr. Sanchez Peña, diseñó un modelo de orden reducido 103 con el cual proponen un controlador de lazo cerrado conmutado [168. Como resultado de la colaboración con nuestro grupo de trabajo, al controlador se le agregó la capa de seguridad SAFE, la cual ya había sido evaluada clínicamente sobre controladores tipo PID en España 163. El agregado de la capa SAFE permitió no sólo prevenir hipoglucemias debido a la sobreactuación del controlador sino que también reducir hiperglucemias utilizando mayores ganancias en el controlador. El algoritmo ARG fue sintonizado bajo intensos ensayos in-silico, incluyendo comidas mixtas incluidas en la plataforma UNLP, y a partir de los mismos se establecieron las reglas de conmutación. [159]. Posteriormente, durante varios encuentros y discusiones entre ambos grupos de trabajo (Fig. 4.5), se terminó de diseñar el algoritmo que se presenta a continuación.

En la Fig. 4.6 se ilustra el sistema de regulación de glucosa a lazo cerrado con el algoritmo ARG para un sujeto $\# j$. De la figura se desprende que el algoritmo está compuesto por dos elementos principales:

- un regulador LQG conmutado (Swithced LQG, SLQG); y 


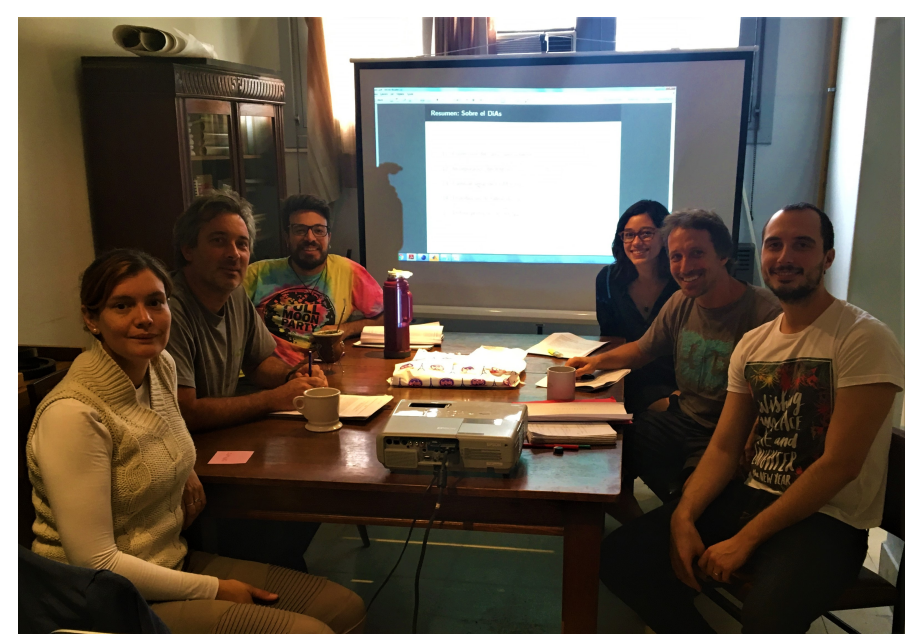

Figura 4.5: Reunión de trabajo junto a, de izquierda a derecha, Dra. Marcela Moscoso, Dr. Hernán De Battista, Ing. Nicolás Rosales, Ing. Emilia Fushimi, Dr. Fabricio Garelli y Dr. Patricio Colmegna.

- la capa de seguridad SAFE.

La señal de referencia $r$ consiste en la concentración de glucosa que se desea alcanzar en situaciones de ayuno. La señal de error $e$ generada por la diferencia entre la concentración deseada y la medida por el sensor CGM es la entrada del algoritmo ARG. Internamente el algoritmo ARG genera una acción de control $u_{\mathcal{C}}$, siendo esta la salida del LQG conmutado (SLQG). Como el controlador no tiene acción integral para evitar la acumulación de insulina, la señal $u_{\mathcal{C}}$ se suma a la infusión de insulina correspondiente al perfil basal del sujeto $i_{b, j}$ para generar la señal $u$. Esta señal de control no es suministrada directamente sino que se ve afectada por el bloque SAFE, ya introducido en este Capítulo en la sección 4.1. La presencia del SAFE, cuya entrada es la señal $u$ y cuya salida es la señal $\gamma$, modula la infusión propuesta por el SLQG a fin de evitar la acumulación excesiva de insulina. La acción de control que finalmente llega a la bomba es $u_{\gamma}$, correspondiendo a la acción de control propuesta por el SLQG $u$ multiplicada por la salida del bloque SAFE $\gamma$.

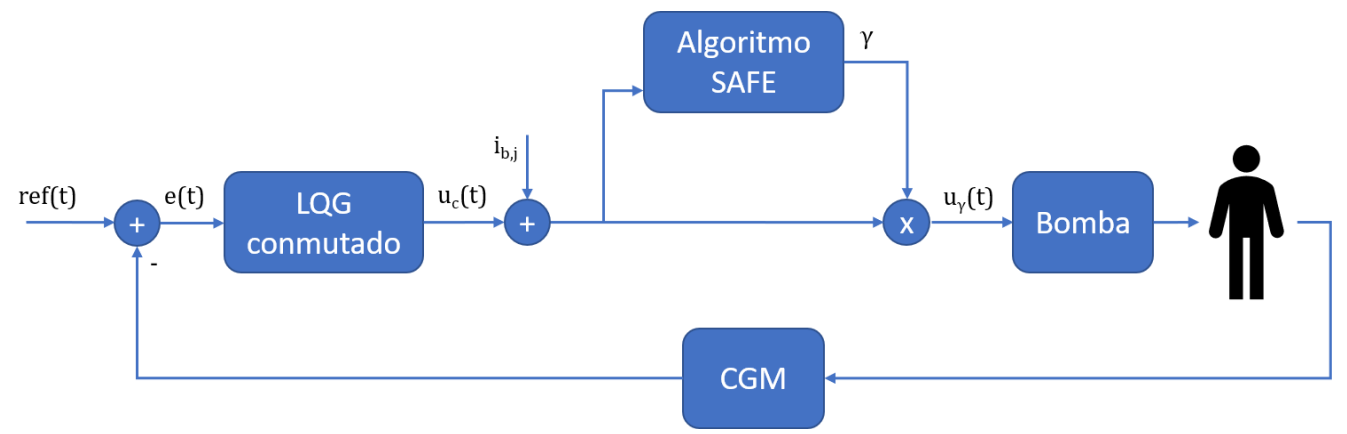

Figura 4.6: Sistema a lazo cerrado con el algoritmo ARG.

\subsubsection{Regulador LQG Conmutado}

El regulador SLQG está formado por 2 reguladores LQG: uno $\mathcal{K}_{1}$ orientado a generar correcciones suaves en la infusión basal de insulina, y otro $K_{2}$ orientado a generar correcciones rápidas y agresivas. La conmutación al controlador $\mathcal{K}_{2}$ ocurre en el momento de las comidas y puede ser manual o automática. Si es manual, el paciente debe anunciar al controlador, por ejemplo pulsando un botón, el instante en que se va a ingerir la comida. Si es automática, debe existir un mecanismo de detección que permita inferir, por ejemplo mediante la señal de CGM, que el sujeto ha comido. 
Debido a que se desea que el algoritmo ARG tenga el menor costo computacional posible en vista a su implementación en una plataforma para su prueba clínica, se opta por la utilización del modelo orientado al control presentado en el Capítulo 2, Sección 2.3 . Así, para cada sujeto \# $j$ se diseñan 2 reguladores $\mathrm{LQG:} \mathcal{K}_{i, j}$ con $i \in \mathcal{I}=\{1,2\}$, basados en el siguiente modelo:

$$
G_{j}(s)=k_{j} \frac{s+z}{\left(s+p_{1}^{*}\right)\left(s+p_{2}\right)\left(s+p_{3}\right)} \mathrm{e}^{-15 s},
$$

el cual es la versión LTI del modelo LPV personalizado (ec. 2.41) en el punto de operación $g=120 \mathrm{mg} / \mathrm{dl}$, i.e. $p_{1}^{*}=p_{1}(120)$.

Como el sensor CGM no envía mediciones en forma continua, sino cada $5 \mathrm{~min}$, el modelo continuo $G_{j}(s)$ se lo representa de forma discreta $G_{j}(z)$ mediante un retenedor de orden cero, y este último se expresa a través de la siguiente realización:

$$
\begin{aligned}
x(k+1) & =A_{j} x(k)+B_{j} u_{\Delta}(k) \\
y_{\Delta}(k) & =C_{j} x(k)
\end{aligned}
$$

con $u_{\Delta}(k)=u(k)-i_{b, j}$, e $y_{\Delta}(k)=y(k)-120 \mathrm{mg} / \mathrm{dl}$. Dada esa realización, se propone un control por realimentación de estados:

$$
u_{\Delta}(k)=-K_{i, j} x(k)
$$

que minimiza el siguiente funcional de costo:

$$
J_{i}\left(u_{\Delta}, y_{\Delta}\right)=\sum_{k=0}^{\infty}\left(R_{i} u_{\Delta}^{2}+Q y_{\Delta}^{2}\right)
$$

con $R_{1}=1, R_{2}=0,5$, y $Q=5 \times 10^{3}$. El parámetro $R_{2}$ se define menor a $R_{1}$ con el fin que $\mathcal{K}_{2, j}(z)$ sea más agresivo que $\mathcal{K}_{1, j}(z)$. Además, los estados se estiman con un filtro de Kalman de la forma:

$$
\hat{x}(k+1 \mid k)=A_{j} \hat{x}(k \mid k-1)+B_{j} u_{\Delta}(k)+L_{i, j}\left[y_{\Delta}(k)-C_{j} \hat{x}(k \mid k-1)\right]
$$

donde $L_{i, j}$ se obtiene asumiendo que los ruidos de proceso $w(k)$ y de medición $v(k)$ son ruido blanco que satisfacen:

$$
E\left[w(k) w(k)^{T}\right]=W_{i}, \quad E\left[v(k) v(k)^{T}\right]=V_{i}
$$

con $W_{1}=V_{1}=W_{2}=3$, y $V_{2}=45 \times 10^{-4}$. Aquí, $V_{2}$ se define menor que $V_{1}$ a fin que $\mathcal{K}_{2, j}(z)$ responda más rápido que $\mathcal{K}_{1, j}(z)$.

De acuerdo a la metodología aplicada, ambos controladores $\mathcal{K}_{1, j}(z)$ y $\mathcal{K}_{2, j}(z)$ tienen una estructura de observador con realimentación de estados, que luego es utilizada para construir el controlador conmutado de acuerdo a la teoría detallada en [169].

Los detalles de la síntesis del controlador y el análisis de estabilidad del mismo se encuentran en [159]. La idea básica es expresar el controlador conmutado de forma tal de poder conmutar arbitrariamente entre ambos controladores LQG sin necesidad de resetear los estados y de una manera muy simple (sólo se conmuta parte de la matriz $C$ del controlador conmutado).

\subsubsection{Capa de Seguridad SAFE}

$\mathrm{Su}$ principal objetivo es modular la ganancia del controlador para evitar que la insulina activa o IOB supere un determinado umbral $\overline{\mathrm{IOB}}(t)$, reduciendo así el riesgo de hipoglucemias en los períodos posprandiales tardíos. A su vez, las características de robustez del 
modo deslizante en que se basa la capa SAFE permiten asignar una ganancia elevada al controlador agresivo $\mathcal{K}_{2, j}$. De esta manera, su rápida acción es rápidamente inhibida por la capa SAFE, generando de forma automática una respuesta no lineal, similar a un perfil de bolo de insulina. La función de conmutación se define es este caso como:

$$
\sigma_{\mathrm{SM}}(t)=\overline{\mathrm{IOB}_{j}}(t)-\operatorname{IOB}(t) .
$$

donde el límite $\overline{\mathrm{IOB}_{j}}(t)$ se define constante a trozos, como será explicado a continuación. El funcionamiento de la misma es tal cual lo definido en la sección anterior de este Capítulo.

Uno de los primeros desafíos en el diseño de la capa fue la selección de los límites de IOB. En principio, sería ideal ajustar el límite de acuerdo a la comida ingerida, pero eso involucraría una estimación exacta de la cuantiad de CHO de la misma por parte del sujeto. A su vez, en vista a la conmutación automática, es necesario depender lo menos posible de las interacciones del sujeto, por lo que, en una primera instancia, se deben acotar las opciones. Si bien pueden existir diversos criterios para definir el límite de IOB, en los ensayos clínicos realizados se definió a partir de una clasificación en el tamaño de las comidas, de la siguiente manera:

- Comidas pequeñas $<35 \mathrm{gCHO} . \overline{\mathrm{IOB}}_{\mathrm{s}, j}(t)=\mathrm{IOB}_{\mathrm{ss}, j}(t)+40 \mathrm{gCHO} / \mathrm{CR}_{j}(t)$.

- Comidas medianas $[35,65) \mathrm{gCHO} \cdot \overline{\mathrm{IOB}}_{\mathrm{m}, j}(t)=\mathrm{IOB}_{\mathrm{ss}, j}(t)+55 \mathrm{gCHO} / \mathrm{CR}_{j}(t)$.

- Comidas grandes $\geq 65$ gCHO. $\overline{\mathrm{IOB}}_{1, j}(t)=\mathrm{IOB}_{\mathrm{ss}, j}(t)+70 \mathrm{gCHO} / \mathrm{CR}_{j}(t)$.

donde $\operatorname{IOB}_{\mathrm{ss}, j}(t)$ es el valor de estado estacionario del modelo (3.7) ante la tasa de insulina basal correspondiente a cada paciente $i_{b, j}(t)$ y XX $\mathrm{CHO} / \mathrm{CR}_{j}(t)$ es el bolo de insulina correspondiente a XX gramos de carbohidratos usando el factor $\mathrm{CR}_{j}(t)$ del paciente al momento de la comida. Cuando el sistema no se encuentra en situación prandial, el límite de IOB se fija como $\overline{\mathrm{IOB}}_{\mathrm{s}, j}(t)$, es decir, el asociado a una comida chica. De esta forma, se le da cierta libertad al controlador a realizar ajustes en la infusión basal cuando sea necesario. Es importante destacar que aunque el paciente debe anunciar el instante de la comida, no se inyecta un bolo preprandial ni debe contar la cantidad los carbohidratos que ingerirá, traduciéndose así en una menor carga en sus tareas diarias.

\subsubsection{Mecanismo de conmutación del multicontrolador}

Como se mencionó anteriormente, la ganancia $\mathcal{K}_{2, j}$ se aplica en periodos posprandiales para generar un pico de insulina, simulando un bolo de la terapia a lazo abierto. Para esto, la ingesta de comidas debe ser detectada y el nivel de IOB debe ser modificado de forma acorde. Podrían tomarse dos enfoques al respecto: anuncio manual de las comidas o su detección automática.

\section{Anuncio de comidas}

En este enfoque sencillo, el sujeto debe anunciar cuando esta próximo a comer, por ejemplo mediante un botón de la plataforma. El uso cualitativo del anuncio de comidas fue discutido en [166] como una manera de ajustar la agresividad de los controladores en ciertas situaciones. Se consideró que el sujeto deba seleccionar entre un rango de comidas y no introducir un valor exacto, ya que el cálculo de $\mathrm{CHO}$ de los alimentos se considera una tarea tediosa para los sujetos. Por lo tanto, se introdujo una clasificación de comidas en pequeñas, medianas y grandes como la arriba descrita. Cuando se produce el anuncio, el algoritmo ARG entra en un modo listening, el cual dura 90 minutos como máximo. Este estado se representa por una variable lógica $l$, siendo la misma igual a 0 por defecto y se ajusta a la unidad cuando se produce el anuncio. Estando en el modo listening, cuando se percibe un 
crecimiento en la tendencia de la glucosa, se selecciona el modo agresivo del controlador SLQG. En este caso, el límite $\overline{\mathrm{IOB}}_{j}(t)$ se cambia a $\overline{\mathrm{IOB}}_{\mathrm{s}, j}(t), \overline{\mathrm{IOB}}_{\mathrm{m}, j}(t)$ o $\overline{\mathrm{IOB}}_{1, j}(t)$ de acuerdo a la clasificación de comidas seleccionada. El modo agresivo permanecerá durante 1 hora. Luego, se conmutará automáticamente al modo conservador nuevamente, y 30 minutos después, el límite de IOB volverá a $\overline{\mathrm{IOB}}_{\mathrm{s}, j}(t)$.

\section{Detección automática de comidas}

$\mathrm{El}$ algoritmo ARG funciona independientemente de como se detecten las comidas. Por lo tanto cualquier algoritmo pre-existente de detección de comidas puede ser implementado 170 174] para generar la conmutación entre $\mathcal{K}_{1, j}$ y $\mathcal{K}_{2, j}$. De hecho, recientemente se publicó la implementación del algoritmo ARG sin anuncio de comidas [175], evaluado de forma in-silico a aprtir de los datos de los ensayos clínicos.

Por lo general, como la detección depende de la señal de CGM, pueden detectarse crecimientos en la excursión de glucosa poco realistas debido al alto ruido de medición. En consecuencia, hay un compromiso entre una velocidad de respuesta y la inmunidad al ruido del CGM. Por esa razón, y debido al hecho de que el algoritmo ARG sería probado en un ensayo clínico por primera vez, no se considera una detección automática en esta primera implementación.

\subsubsection{Módulos auxiliares}

Dentro del diseño de controladores de lazo cerrado, es sabido el hecho de que existe una gran dificultad para lograr un control seguro de la glucosa en sangre en la DMT1. Esto se debe a la naturaleza variable en el tiempo y a la gran incertidumbre de la dinámica del sistema glucosa-insulina. Como se describió en el Capítulo 1 Sección 1.3.1 y puede observarse en la Fig. 1.13 de esa misma sección, para minimizar los riesgos de hipo- e hiperglucemia, se consideran módulos auxiliares. En este caso, se consideran dos módulos, los cuales se activan sólo cuando es necesario ajustar la acción de control comandada por el algoritmo ARG porque se detecta un evento inminente de hipo- o hiperglucemia.

\section{Módulo relacionado con la hipoglucemia}

En caso que se detecten niveles bajos de glucosa, se propone un algoritmo que disminuya el valor del límite de IOB de la capa SAFE, por lo tanto, se suspenderá o atenuará el suministro de insulina que entregue el controlador. El módulo funciona de la siguiente forma:

1: En cada tiempo de muestreo:

2: Se recibe la glucosa medida por el sensor CGM ( $g[\mathrm{mg} / \mathrm{dl}])$, y se realiza una extrapolación lineal para estimar la tasa de cambio de la glucosa $\left(\hat{\dot{g}}_{30}[\mathrm{mg} / \mathrm{dl} / \mathrm{min}]\right)$, y se predice el nivel de glucosa en un horizonte de $15 \mathrm{~min}\left(\hat{g}_{15}\right)$, considerándose las últimas 6 muestras de CGM (últimos 30min).

3: El límite de IOB se ajusta de acuerdo a las secciones anteriores.

4: if $g<60$ then

5: $\quad \overline{\mathrm{IOB}}_{j}(t)=0$

6: else if $g<70$ then

7: $\quad \overline{\mathrm{IOB}}_{j}(t)=0,5 \mathrm{IOB}_{\mathrm{ss}, j}(t)$

8: else if $i=1$ and $l=0$ then

9: $\quad$ if $\hat{\dot{g}}_{30}<-0,5 \mathbf{o}\left[\hat{\dot{g}}_{30}<0,5 \mathbf{y} \operatorname{IOB}(t) \geq \operatorname{IOB}_{\mathrm{ss}, j}(t)\right]$ then

10: $\quad$ if $\hat{g}_{15}<70$ then

11: $\quad \overline{\mathrm{IOB}}_{j}(t)=0,5 \mathrm{IOB}_{\mathrm{ss}, j}(t)$

12: $\quad$ else if $\hat{g}_{15}<100$ then 


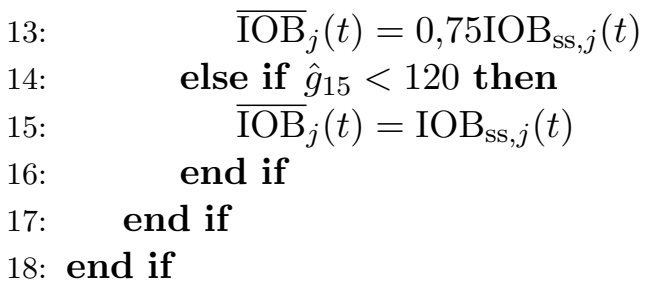

Debe notarse que el código a partir de la línea 8 sólo se ejecutará si el controlador se encuentra en modo conservador $(i=1)$ y no si se encuentra en modo listening $(l=0)$. En caso de que se active este módulo, la variable lógica HYPoFlag será igual a 1, de otro modo, permanecerá en 0 .

\section{Módulo relacionado con la hiperglucemia}

Este modulo se utiliza cunado el sujeto, sin estar en un periodo posprandial y ante el suministro de insulina basal, esté sin embargo un nivel alto de glucosa (no necesariamente hiperglucemia). Este módulo genera un bolo de corrección automático (BAC) en [U] a partir del CF del sujeto cuando existe una excursión hiperglucémica persistente que no puede ser corregida por el controlador en modo conservador.

1: En cada tiempo de muestreo:

2: De la misma manera que se estimaron la tasa de cambio $\left(\hat{\dot{g}}_{30}\right)$ y la futura concentración de glucosa $\left(\hat{g}_{15}\right)$ en el módulo anterior, se consideran en este móduld ${ }^{1}$

3: Se calcula el valor medio de las últimas 6 muestras de CGM $\left(\bar{g}_{30}\right)$.

4: La variable lógica G160FlaG, la cual es 0 por defecto, toma el valor de la unidad si las últimas 6 muestras de CGM son mayores a $160 \mathrm{mg} / \mathrm{dl}$.

5: Se determinan los contadores CCBolus y CAGGCon los cuales cuentan los minutos entre el último BAC y la última conmutación de agresivo a conservador del controlador, respectivamente.

6: if $i=1 \mathbf{y} l=0$ then

7: if HYPOF $_{\mathrm{LAG}}=0$ and $\mathrm{CCBOLUS} \geq 120$ and $\mathrm{CAGGCON} \geq 180$ then

8: $\quad$ if $\left(\right.$ G $\left.160 F_{L A G}=1 \mathbf{y} \hat{\dot{g}}_{30} \geq 0 \mathbf{y} \hat{\dot{g}}_{15} \geq-0,5\right)$ o $\bar{g}_{30}>200$ then

9: $\quad \mathrm{BAC}=0,8\left[\min \left(\bar{g}_{30}, \hat{g}_{15}\right)-120\right] / \mathrm{CF}$

10: $\quad$ end if

11: $\quad$ end if

12: end if

Finalmente el límite de IOB de la capa SAFE se aumenta en 0,8·BAC. Obsérvese que según las condiciones que deben cumplirse para la generación de un BAC es probable que este módulo se active sólo durante los períodos de ayuno con una hiperglucemia persistente. Se considera el valor mínimo entre $\bar{g}_{30}$ y $\hat{g}_{15}$ ya que el ruido de medición puede dar como resultado la sobre-estimación del BAC. Por esto mismo, se considera un factor conservador de 0,8 para su cálculo. Aún así, el BAC se agrega al bolo de insulina propuesto por el ARG de forma tal que la cantidad de insulina total a suministrar pasa por la capa SAFE, pero no se toma en cuenta en la actualización de los estados del controlador. Lo primero es por seguridad, y lo segundo, para que no genere una reducción en la infusión posterior propuesta por el controlador.

\subsubsection{Ejemplo de operación}

La Figura 4.7 muestra como funciona el algoritmo ARG. Se puede ver la respuesta ante la ingesta de una comida mixta de la plataforma UNLP (leche, pan blanco, queso bajo en

\footnotetext{
${ }^{1}$ Se agrega una estimación de la tasa de cambio a partir de las últimas 3 muestras (15min) de CGM $\left(\hat{\dot{g}}_{15}\right)$, ya que $\hat{\dot{g}}_{30}$ podría ser nula pero $\hat{\dot{g}}_{15}$ indicar una tendencia marcadamente negativa
} 
grasa, manteca y aceite; $\mathrm{CHO}=110 \mathrm{~g})$. En particular, la tasa de aparición $\left(R_{a}\right)$ de esta comida presenta dos picos, uno cuando se ingiere la comida, y otro unas 3 horas más tarde. El suministro de insulina provisto por el controlador depende en la mayor parte del tiempo de $\mathcal{K}_{1}$. En el periodo posprandial, se conmuta a $\mathcal{K}_{2}$, proveyendo bolos de insulina mayores para evitar la hiperglucemia. Puede observarse que estos bolos son modulados por la capa SAFE mediante la ganancia $\gamma$ para evitar violar la restricción impuesta en el IOB. De esta forma, se evita una posible hipoglucemia por el exceso de insulina. A su vez, puede verse la acción de los módulos auxiliares sobre el límite de IOB. Cuando se predicen posibles excursiones hipoglucémicas, el límite de IOB se baja. Por otro lado, cabe señalar pasado el modo agresivo la acción de un BAC para complementar la acción del modo conservador en consecuencia del segundo pico de la tasa de aparición de glucosa de la comida.

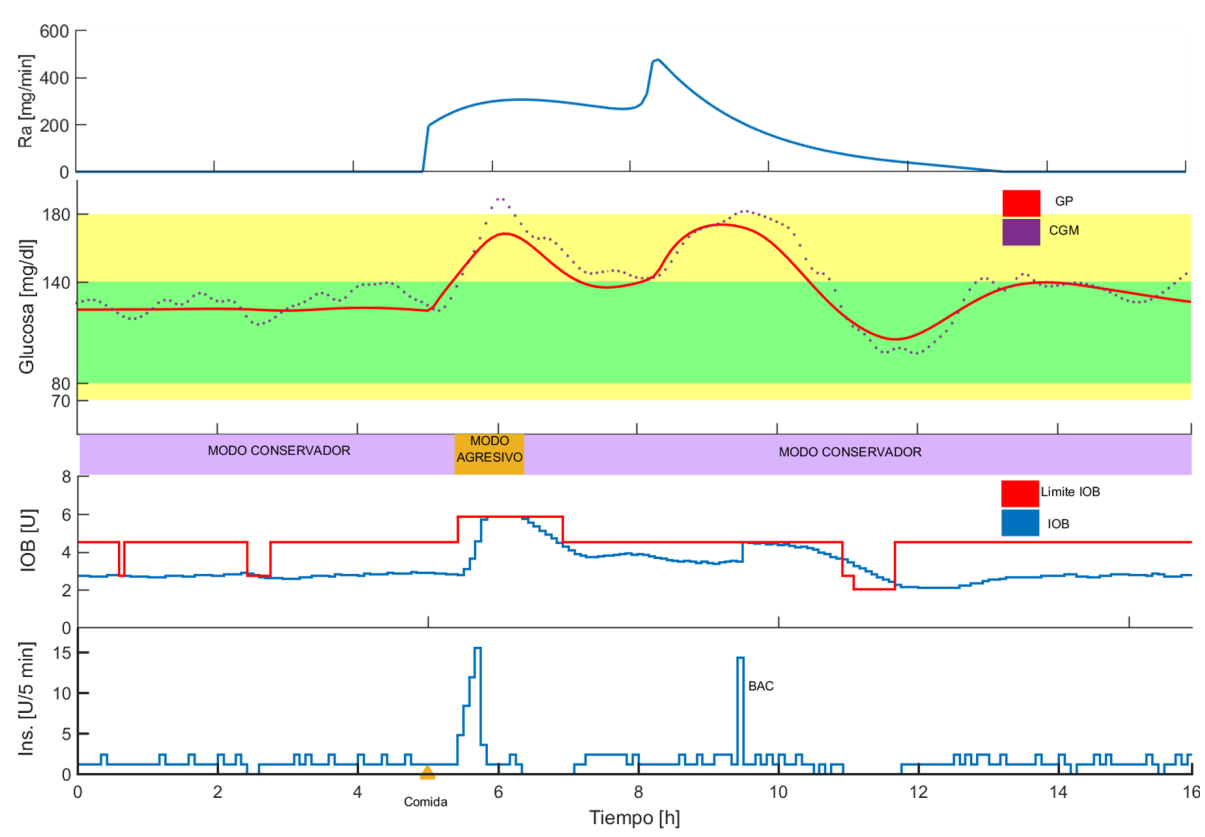

Figura 4.7: Respuesta de lazo cerrado del algoritmo ARG para un adulto in-silico ante la ingesta de una comida mixta.

En el trabajo publicado en [159] pueden verse los resultados de los ensayos in-silico realizados para validar la estrategia de control antes de ser ensayada clínicamente.

\subsubsection{Pruebas Clínicas}

Las primeras pruebas clínicas con un sistema de $\mathrm{PA}$ en Latinoamérica se llevaron a cabo en dos etapas en el HIBA. Una primera etapa fue durante noviembre de 2016 con el algoritmo de control desarrollado en la Universidad de Virginia [158, 176], que ya había sido probado en numerosos centros alrededor del mundo [157], y una segunda etapa en junio de 2017 con el algoritmo ARG desarrollado en Argentina. Ambas etapas contaron con la misma cantidad de pacientes conDMT1 (5) y con el mismo sistema de comunicación entre componentes. Sin embargo, la principal diferencia es que en la segunda etapa el paciente no se debía aplicar el bolo de insulina previo a las comidas como en la terapia convencional, sino que sólo debía anunciar el instante en que iba a comer.

\section{Diseño del estudio y participantes}

Para la realización de los ensayos clínicos se tuvo que realizar un protocolo, el cual debió ser aprobado tanto por el comité de ética del Hospital Italiano de Buenos Aires 
(HIBA) como por la Administración Nacional de Medicamentos, Alimentos y Tecnología Médica (ANMAT). A continuación se describen los principales aspectos de este protocolo.

En primer lugar, la selección de los 5 participantes se basó en los siguientes criterios de inclusión y exclusión:

\section{- Criterio de inclusión}

- Haber sido diagnosticados con DMT1 hace al menos 2 años y que estuviesen utilizando bomba de insulina con sensor de monitoreo continuo de glucemia por un período no menor a 6 meses previos al inicio del estudio.

- Edad entre 18 y 65 años.

- $\mathrm{HbA} 1 \mathrm{c}>6.5 \% \mathrm{y}<10 \%$.

- Aquellas mujeres que fuesen potencialmente fértiles debían utilizar efectivos métodos contraceptivos y presentar B-HCG negativa en el laboratorio de screening.

- Adecuado estado mental y cognitivo como para comprender las consideraciones del estudio y para el manejo de los componentes del sistema cerrado.

- Pacientes entrenados en el conteo de carbohidratos.

- Individuos que hubiesen comprendido el presente protocolo y hubiesen firmado el Formulario de Consentimiento Informado.

\section{- Criterio de exclusión}

- Haber sido hospitalizados por cetoacidosis diabética en los últimos 12 meses.

- Haber presentado hipoglucemias severas con pérdida de consciencia en los últimos 12 meses.

- Presentar alguna enfermedad coronaria.

- Padecer hipertensión arterial no controlada.

- Presentar cualquier otra condición que a criterio del investigador aumente el riesgo de hipoglucemia.

- Sospecha o diagnóstico de proceso infeccioso activo.

- Fibrosis quística.

- Mujeres embarazadas, con intención de quedar embarazadas o en lactancia.

- Haber estado hospitalizados para tratamiento psiquiátrico en los últimos 6 meses.

- Sospecha o diagnóstico previo confirmado de enfermedad adrenal.

- Presentar alteración hepática con transaminasas elevadas $>2$ veces por encima del VSN.

- Presentar función renal disminuida, con $\mathrm{FG}<60 \mathrm{ml} / \mathrm{min} / 1.73 \mathrm{~m}^{2}$.

- Insuficiencia cardíaca congestiva descompensada o sintomática actual.

- Padecer gastroparesia activa, definida como sintomatología compatible con gastroparesia por más de tres meses al menos tres veces por semana.

- Uso de paracetamol en los últimos 72 horas previas al estudio.

- Uso de antidiabéticos orales e incretinas.

- Paciente en tratamiento por enfermedad oncológica. 
Los participantes consistieron en 3 mujeres y 2 hombres, con una edad media $43 \pm 6$ años, TDI de $38.1 \pm 13.5 \mathrm{U}$, HbA1c de $7.4 \pm 0.7 \%$, peso de $65.3 \pm 15.8 \mathrm{~kg}$ y una duración de su diabetes de $19 \pm 5$ años.

Para la implementación del sistema de $\mathrm{PA}$ en estas pruebas se utilizó para cada sujeto un sensor CGM Dexcom G4 Share ${ }^{\circledR}$, una bomba de insulina Accu-Check Combo ${ }^{\circledR}$ de Roche y un smartphone basado en Android con el sistema Diabetes Assistant (DiAs) [177]. En la Fig. 4.8 puede verse a los 5 participantes con su respectivo smartphone durante el ensayo.

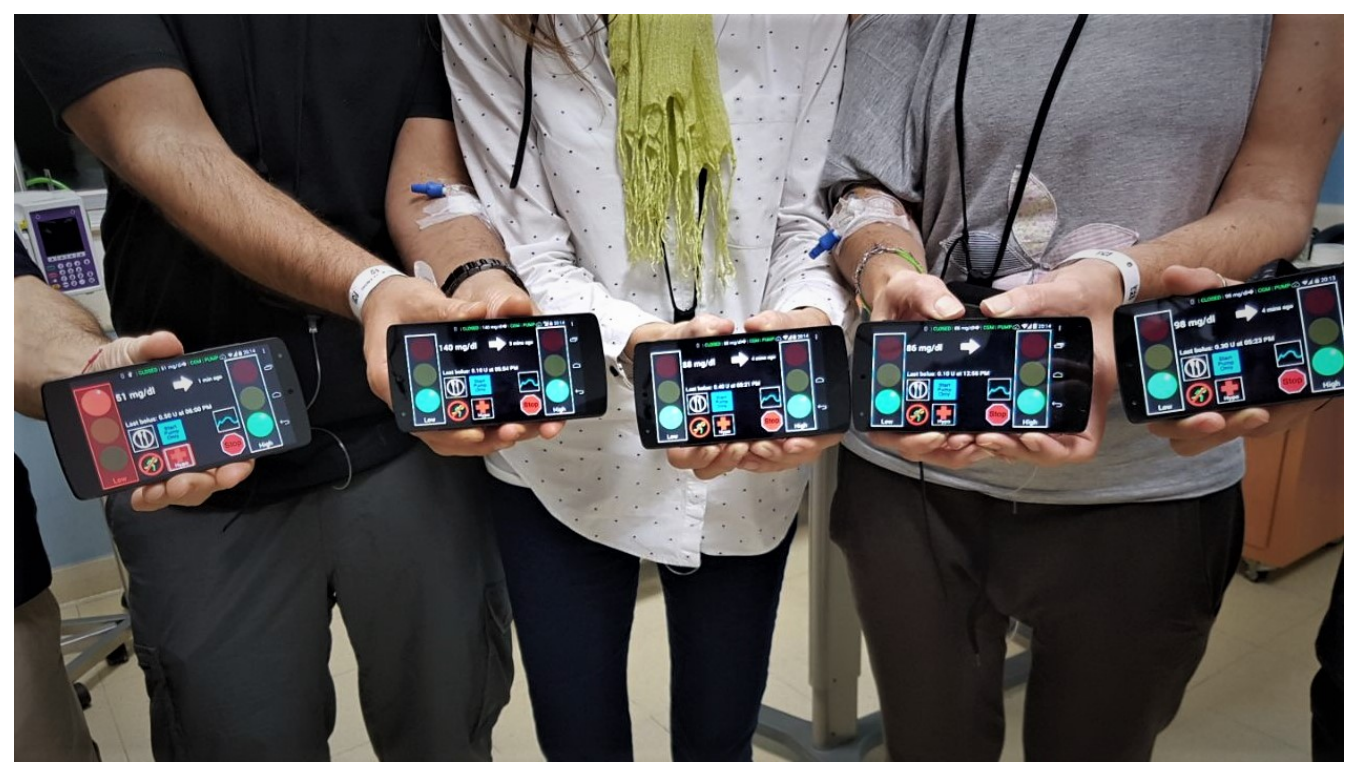

Figura 4.8: Los 5 participantes con sus respectivos smartphones con la plataforma DiAs y el algoritmo ARG corriendo en ella.

El sistema DiAs es una plataforma que permite cada 5 minutos la comunicación de un algoritmo de control con el sensor CGM (con Dexcom por Android Bluetooth Low Energy) y la bomba insulina (con Roche por Bluetooth clásico). Dado que el objetivo general del sistema operativo Android no está relacionado con aplicaciones médicas, para que sea aceptado por la FDA como un dispositivo médico Clase III se removieron las funciones de llamadas, navegador, Android Market, juegos, música y demás. Además, el sistema cuenta con una base de datos SQLite para el manejo de datos, y con actividades y servicios que pueden operar de manera asincrónica en respuesta a la interacción del usuario con el sistema. El sistema DiAs es un sistema modular, en donde el algoritmo de control representa uno de dichos módulos. Para la migración del algoritmo ARG al DiAs, se debió escribir el código bajo el paradigma de la programación orientada a objetos, en lenguaje Java y JavaScript, lo que permitió encapsular el código facilitando el proceso. Una vez migrado el código, luego sólo restó compatibilizar las entradas y salidas.

La linea temporal del protocolo se presenta en la Fig. 4.9. Dos días antes del ensayo, se le proporcionó a cada sujeto los dispositivos para que continúen su tratamiento habitual (tratamiento a LA) en sus hogares hasta el día del ensayo a LC. Durante ese tiempo, se guardó la información de tratamiento de la diabetes en la base de datos. El día de la admisión, los sujetos se reunieron con el equipo de investigación (tanto médicos como ingenieros) en el HIBA a las 16:00 hs, sin restricción de su dieta. Una vez revisado el protocolo de admisión nuevamente, se emparejaron los dispositivos con la plataforma DiAs. Luego de las 19:00 hs, se cerró el lazo de control. Los sujetos estuvieron a lazo cerrado mientras recibieron la cena inicial del día 0 y el desayuno, almuerzo, merienda y cena del día 1, mientras realizaban algunas actividades de esparcimiento. Los mismos debían permanecer en el establecimiento, pero tuvieron la posibilidad de deambular o realizar 


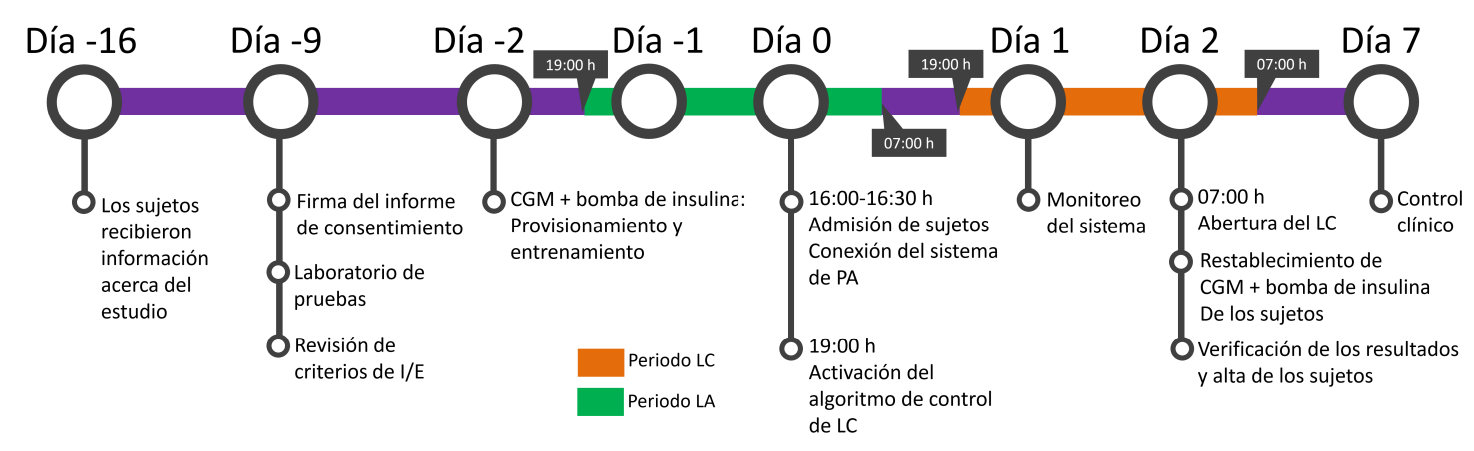

Figura 4.9: Línea de tiempo del protocolo realizado durante los ensayos clínicos.

tareas en un rango no mayor de 100 metros a la redonda. El día 1 se realizó una caminata de 30-45 minutos dentro del hospital. Se evaluó la respuesta del sistema de lazo cerrado ante el aumento de la glucemia y el tiempo hasta la corrección, analizando la respuesta del controlador en modo agresivo y en modo conservador. A su vez, se evaluó la acción de la capa SAFE para luego sacar conclusiones respecto al diseño de los límites de IOB.

Antes de cada comida y dos horas después cada comida se realizaron nuevos controles de glucemia capilar por automonitoreo. Los sujetos podían tomar agua libremente y realizar ingestas adicionales si era necesario por los controles de glucosa. Durante las 2 noches se realizó el monitoreo remoto del funcionamiento del sistema. Un equipo conformado por médicos e ingenieros mantenía la guardia durante el trascurso de las 36 horas de ensayo, asegurando las calibraciones de CGM diarias (7 calibraciones aproximadamente) y proveían de $15 \mathrm{~g}$ de $\mathrm{CHO}$ de rescate en caso de que la glicemia caiga por debajo de $70 \mathrm{mg} / \mathrm{dl}$.

Finalmente, a las 8:00 hs del día 2 se procedió a la apertura del lazo, dando por concluido el ensayo. Se realizó un control de glucemia capilar por automonitoreo al final del período de evaluación y luego del mismo se autorizó a cada sujeto a desayunar. Previo al alta, se restablecieron los mecanismos de infusión de insulina que cada sujeto presentaba antes del estudio. Luego de verificar los resultados y realizar los controles clínicos pertinentes, se autorizó el alta hospitalaria de los participantes.

Los sujetos sólo debían anunciar el tamaño de la comida antes de ingerirla (chica, mediana o grande). El menú fue acordado con la nutricionista, donde se priorizó comidas de lenta aparición de glucosa:

\section{- Desayuno y merienda}

\begin{tabular}{lll} 
Alimento & Cantidad $(\mathrm{g})$ & Cantidad de hidratos $(\mathrm{g})$ \\
\hline Infusión (té, café o mate cocido) & $\mathrm{C} / \mathrm{N}$ & - \\
Pan integral fargo o 5 galletitas tipo de agua & $50(2$ unidades $)$ & 20 \\
Dulce diet & $8(1$ pote $)$ & - \\
Queso untable & 20 & - \\
\hline Total & & 28
\end{tabular}

- Cena

\begin{tabular}{lll} 
Alimento & Cantidad $(\mathrm{g})$ & Cantidad de hidratos $(\mathrm{g})$ \\
\hline Pasta integral Knorr con salsa fileto natural & $50($ crudo) & 40 \\
Carne magra & 100 & - \\
Fruta fresca & 1 unidad & 15 \\
\hline Total & & 55
\end{tabular}

\section{- Almuerzo}

\begin{tabular}{lll} 
Alimento & Cantidad $(\mathrm{g})$ & Cantidad de hidratos $(\mathrm{g})$ \\
\hline Puré de papas con agua $(285 \mathrm{cc})$ & $200(2$ unidades $)$ & 40 \\
Carne magra & 100 & - \\
Fruta fresca & 1 unidad & 15 \\
\hline Total & & 55
\end{tabular}




\section{Resultados}

El propósito de este estudio clínico fue obtener la validación clínica del algoritmo de ARG probando su capacidad para mantener la concentración de glucosa del sujeto en un rango seguro durante 36 horas sin bolos de insulina antes de las comidas. La condición previa para que el controlador ARG fuera verificado era que el sistema funcionara correctamente al menos el $80 \%$ del tiempo total de conexión. Los resultados secundarios fueron el porcentaje de tiempo total dentro del rango deseable de glucemia $(70-180 \mathrm{mg} / \mathrm{dl})$; el porcentaje de tiempo total dentro del rango aceptable de glucemia (70-250 mg/dl); el porcentaje de tiempo total en hipoglucemia $(<70 \mathrm{mg} / \mathrm{dl})$; porcentaje del tiempo total en la hiperglucemia (>180 mg/dl); número de episodios hipoglucémicos sintomáticos y asintomáticos; y comparación de los registros glucémicos obtenidos durante el ensayo con los registros antes de la hospitalización. Esto último no se pudo realizar de forma rigurosa, ya que el protocolo durante LC no fue el mismo que a LA. Aún así, esto no resulta un impedimento ya que el objetivo de este estudio era validar el algoritmo de control ARG (estabilidad, conexión y desempeño de control glucémico).

Para el análisis de los resultados, se definieron diferentes periodos:

- $36 \mathbf{~ h}$ (intervalo total)

LA: 21/06 19:00 h a 23/06 07:00 h.

LC: 23/06 19:00 h a 25/06 07:00 h.

- 15 h (últimas horas de LC)

LA: 22/06 16:00 h a 23/06 07:00 h.

LC: $24 / 06$ 16:00 h a 25/06 07:00 h.

- $\mathbf{N}_{1}$ (1ra noche)

LA: $21 / 06$ 23:00 h a 22/06 07:00 h.

LC: 23/06 23:00 h a 24/06 07:00 h.

- $\mathbf{N}_{2}$ (2da noche)

LA: $22 / 06$ 23:00 h a 23/06 07:00 h.

LC: $24 / 06$ 23:00 h a 25/06 07:00 h.

Dado que era la primera vez que se probaba el algoritmo ARG con pacientes reales, se utilizaron las primeras 3 comidas para realizar los ajustes necesarios del límite máximo de IOB. Es por eso que también se realiza un análisis de los resultados en las últimas 15 horas en LC, y su comparación con 15 horas a lazo abierto que involucren el mismo período del día. El límite de IOB estándar de cada sujeto fue intencionalmente incrementado para el primer almuerzo con la intención de probar la respuesta del controlador, y esa sintonización resultó ser demasiado agresiva.

Los resultados detallados del ensayo clínico pueden apreciarse en [20]. En resumen, con el algoritmo ARG se alcanzó un índice LBG ${ }^{2}$ medio $\leq 2.5$, indicando un bajo riego de hipoglucemia 178. Además, el tiempo medio en $<70 \mathrm{mg} / \mathrm{dl}$ fue de $5.8 \%$ y $4.1 \%$ para las 36 h y 15 h, respectivamente, y $1.0 \%$ y $5.0 \%$ para las noches $\mathrm{N}_{1}$ y $\mathrm{N}_{2}$, respectivamente. Por otro lado, se obtuvo un bajo índice medio de hiperglucemia $\mathrm{HBGI}^{2} \leq 4.5$ para el periodo de $15 \mathrm{~h}$ y noche $\mathrm{N}_{2}$, y un riego moderado HBGI $\leq 9$ para el periodo de $36 \mathrm{~h}$ y noche $\mathrm{N}_{1}$. Además, el porcentaje medio de tiempo en el rango $>180 \mathrm{mg} / \mathrm{dl}$ fue del 19,5\% para el período de $36 \mathrm{~h}$, del $13,3 \%$ para el período de $15 \mathrm{~h}$, y del $27,9 \%$ y $7,3 \%$ para las noches $\mathrm{N}_{1}$ y $\mathrm{N}_{2}$, respectivamente. En términos de la proporción de tiempo en los rangos deseados y aceptables de glucemia durante todo el período de $36 \mathrm{~h}$, la administración de insulina de LC dio un porcentaje medio de tiempo en el rango de $70-250 \mathrm{mg} / \mathrm{dl} \mathrm{de}$ $88,6 \%$, y en el rango de $70-180 \mathrm{mg} / \mathrm{dl}$ de $74,7 \%$. Durante el período de $15 \mathrm{~h}$ se obtuvieron mayores aumentos en el tiempo medio porcentual en los rangos de $70-250 \mathrm{mg} / \mathrm{dl}(94,7 \%)$ y $70-180 \mathrm{mg} / \mathrm{dl}(82,6 \%)$. Nótese que hubo una tendencia hacia una menor hiperglucemia 
en el curso del estudio del LC, mientras que se logró un bajo riesgo de hipoglucemia en todos los intervalos de tiempo.
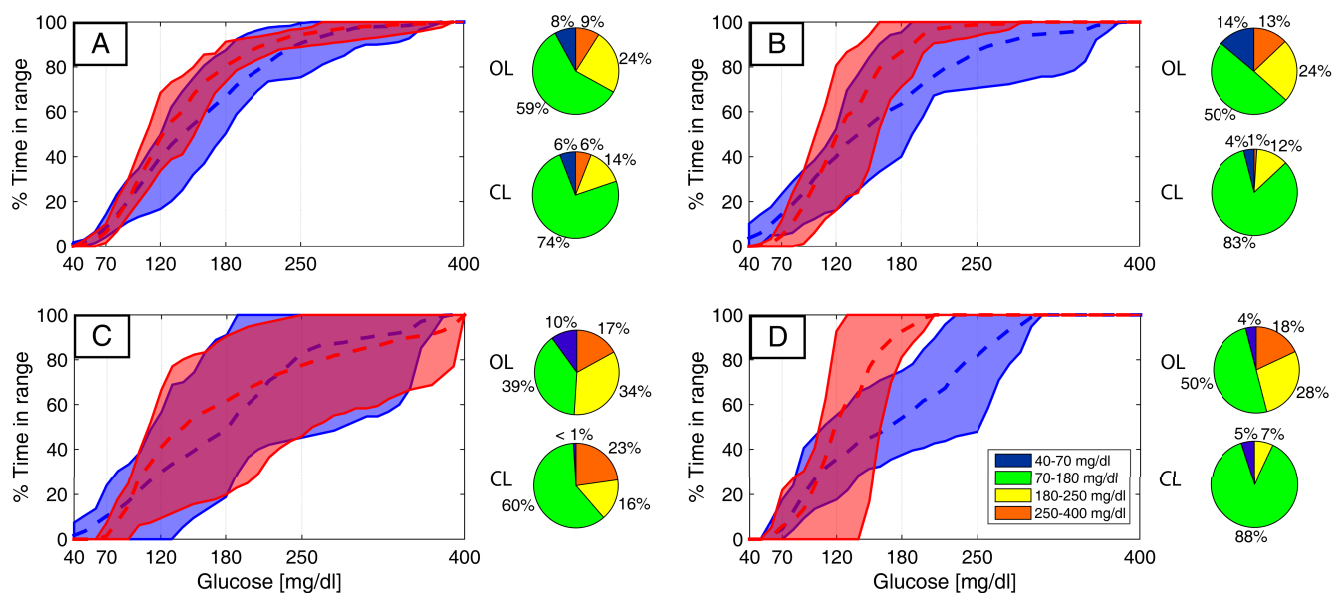

Figura 4.10: Tiempo en rango cumulativo para los 5 sujetos en LA (azul) y LC (rojo) para los periodos de 36 horas (A), 15 horas (B), y las noches 1 (C) y 2 (D). Las líneas punteadas indican los valores medios y las líneas continuas con sombreado indican \pm desviación estándar.

En cuanto al control nocturno, donde se puede realizar una comparación entre las estrategias de LC y LA, el aumento del tiempo medio porcentual en el rango de 70-250 $\mathrm{mg} / \mathrm{dl}$ durante la noche $\mathrm{N}_{1}$ fue del $17,6 \%(\rho=0,176)$, y en el rango de $70-180 \mathrm{mg} / \mathrm{dl}$ fue del $44,7 \%$ ( $\rho=0,072)$. Se obtienen resultados similares cuando se analiza la noche $\mathrm{N}_{2}$. En ese caso, el aumento del tiempo medio porcentual en el rango $70-250 \mathrm{mg} / \mathrm{dl}$ fue del 16,9\% $(\rho=0,341)$, y en el rango $70-180 \mathrm{mg} / \mathrm{dl}$ fue del $37,4 \%(\rho=0,035)$. Para ilustrar este análisis, el promedio, el mínimo y el máximo de tiempo acumulado en el rango se presentan en la Fig. 4.10, y las respuestas de tiempo promedio en LA y LC para el período de $15 \mathrm{~h}$ se representan en la Fig. 4.11. Cabe destacar que la concentración media de glucosa a las 07:00 h del día 2 (cuando se abrió el LC) fue de $120 \mathrm{mg} / \mathrm{dl}$, lo que coincide exactamente con el punto de ajuste del LC.

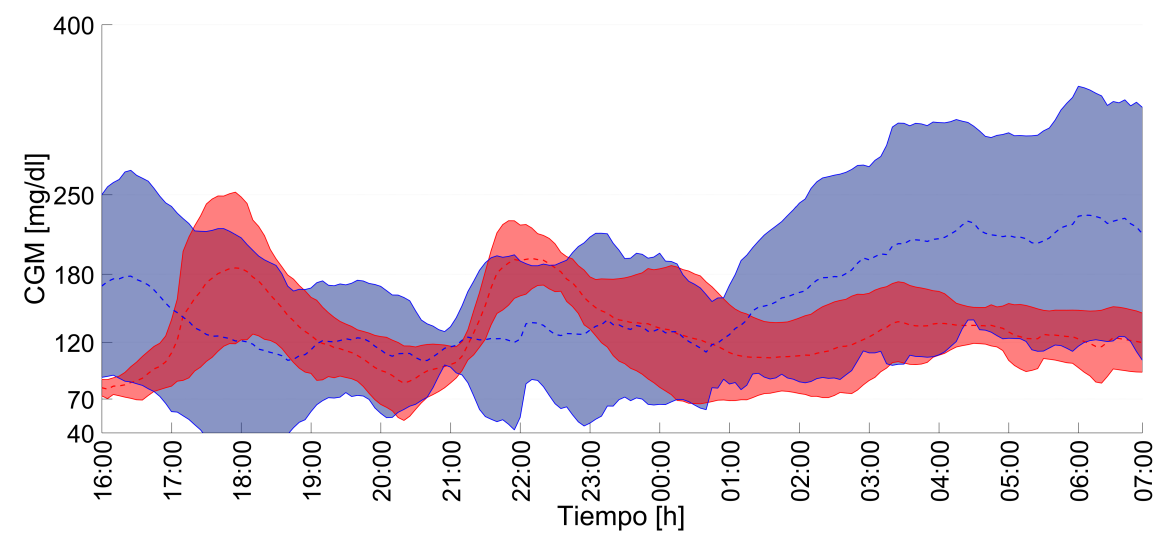

Figura 4.11: Excursión promedio de CGM para los 5 sujetos en LA (azul) y LC (rojo) durante el periodo de 15 horas (media \pm DS).

\footnotetext{
${ }^{2}$ Los índices Low Blood Glucose Index (LBGI) y High Blood Glucose Index (HBGI) son métricas populares que se utilizan para cuantificar el riesgo de hipo- e hiperglucemia. Se diseñaron sobre la base de una simetrización del rango de la GP para resumir el número y el alcance de las fluctuaciones extremas de la GP en números individuales, con el LBGI para los episodios hipoglucémicos y el HBGI para los hiperglucémicos. Por lo tanto, un LBGI más alto puede indicar un gran número de episodios hipoglucémicos leves, un pequeño número de episodios hipoglucémicos graves, o una combinación de ambos, y lo mismo puede decirse del HBGI con respecto a hiperglucemia 179
} 
Hay numerosos estudios y revisiones sobre sistemas híbridos de PA, pero pocos se basan en algoritmos sin bolos de insulina. El control de la glucosa posprandial es un gran desafío usando un sistema de control de glucosa completamente automatizado. Por lo tanto, se decidió probar el controlador ARG por pasos. En este primer paso, se le informó al controlador de la hora de la comida y de la clasificación por tamaño de la comida. Esto permitió reducir el número de sistemas a probar simultáneamente. En una futura prueba clínica, cualquier estrategia de detección de comidas podría ser empleada, porque el controlador de LC es independiente de cómo se detectan las comidas. El uso de una clasificación del tamaño de las comidas se puede encontrar en otros trabajos también 180,181. En este caso, la clasificación se usó para ajustar el límite de IOB a través del CR del sujeto, pero no para generar un bolo de insulina relacionado con la comida. Cuanto más alto es el límite de IOB, menos restrictiva es la capa de seguridad. Si se utilizara un único tamaño de comida estándar para definir el límite de IOB para cada comida, ese límite podría ser demasiado alto para las comidas pequeñas o demasiado bajo para las comidas grandes. Por lo tanto, este esquema de clasificación de tres comidas añadió un grado extra de libertad al algoritmo ARG, permitiendo reducir la incertidumbre en la restricción del IOB. En una próxima etapa, esta restricción podría ser adaptada automáticamente basada en un algoritmo de estimación de carbohidratos.

\subsection{Conclusiones}

En este Capítulo se presentaron algoritmos de control de lazo cerrado para el control de glucosa. En primer lugar el algoritmo Time Enable es una primera aproximación a la protección ante posibles hipoglucemias en configuraciones híbridas de control glucémico. Esta propuesta permite que la excursión de la glucosa en sangre sea menos sensible a los cambios de ganancia del controlador principal. Por lo tanto, se puede afirmar que la utilización de la capa de seguridad permite probar un controlador cuya sintonización no se encuentre ajustada, sin exponer al sujeto a episodios hipoglucémicos graves.

Por otro lado, se ppresentaron las ideas fundamentales del controlador ARG y los resultados de los primeros ensayos clínicos utilizando un sistema de PA en América Latina. El algoritmo ARG permite ser personalizado a partir de información clínica de los sujetos de forma a priori, y presenta la ventaja de no requerir el conteo de carbohidratos, un aspecto muy valorado por los participantes del ensayo. Esta etapa sirvió para un gran enriquecimiento en cuanto al trabajo colaborativo, diseño y ejecución de ensayos clínicos, dando lugar a la participación de futuras realizaciones en el ámbito ambulatorio o en poblaciones pediátricas. 


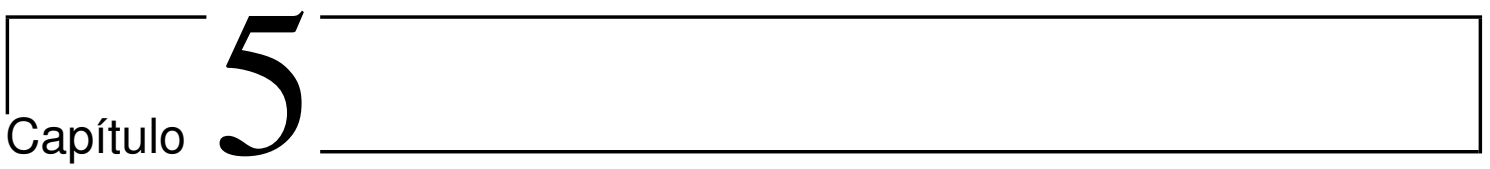

\section{Protección frente a hipoglucemias a lazo-cerrado vía limitación de la tasa de decrecimiento de la glucosa}

La principal propuesta de este Capítulo es el algoritmo GSAFE (Glucose-based Safety Auxiliary Feedback Element). Este algoritmo consiste en una capa de control que actúa como protección ante hipoglucemias. Tiene un comportamiento predictivo y puede asociarse a los sistemas Predictive Low Glucose Suspend (ㅍG) utilizados hoy en día en los tratamientos SAP. La diferencia con los sistemas actualmente implementados es que la administración de insulina no se suspende y se reanuda. Lo que se propone es adaptar la salida del controlador para cumplir con una cierta restricción impuesta a la tasa de decrecimiento de la glucosa para así evitar la hipoglucemia. Además, a diferencia de la protección ante hipoglucemias propuesta anteriormente por los directores de la tesis [141] y presentada en el Capítulo 4, la que se hace aquí está pensada para enfoques de lazo cerrado puro sin necesidad de anuncio de comida. La propuesta fue presentada en el congreso internacional ATTD 2018 182 y enviada a la revista de alto impacto IEEE Journal of Biomedical and Health Informatics.

\subsection{Sistemas de prevención de hipoglucemias}

Aunque los recientes avances en el manejo de la diabetes mejoraron la calidad de vida de los sujetos diabéticos, el mantenimiento de un control glucémico estricto aumenta el riesgo de hipoglucemia 183 . Esto se debe principalmente a las dinámicas y retardos que conducen a la sobreactuación (exceso de insulina). Muchos dispositivos CGM incorporan un sistema de alarma para advertir al sujeto de una eventual hipoglucemia [184]. Por ejemplo, el enfoque en 185 utiliza 5 algoritmos para predecir la hipoglucemia: proyección lineal, predicción estadística, filtro de Kalman, respuesta a impulsos finitos y algoritmos numéricos lógicos. Luego, la alerta predictiva de un evento hipoglucémico se realiza mediante un algoritmo de votación. El problema del recurso de alarma es que los usuarios no responden durante el sueño o tienden a ignorarlos debido a las falsas alarmas durante el día. Hoy en día los sistemas SAP con algoritmos predictivos para prevenir valores bajos de GP mediante la suspensión del suministro de insulina tienen el potencial de reducir la exposición a la hipoglucemia. Esta clase de algoritmos aplicados a la terapia SAP se los denomina sistemas LGS 186.

El objetivo de los sistemas de LGS es el de evitar la hipoglucemia, interrumpiendo parcial o totalmente el suministro de insulina. Estos sistemas pueden ser simples, de modo 
que la suspensión total de insulina se produce cuando se alcanza un umbral específico, o tener un enfoque predictivo, en donde el suministro se detiene cuando se predice que se va a violar un determinado umbral [187]. Estos últimos se los refiere también como sistemas PLGS, Si bien estos sistemas no corresponden a un control de lazo cerrado puro los sistemas PLGS representan los primeros pasos en la ruta hacia el sistema de PA (como puede observarse en la Fig. 1.12 de la sección introductoria) como lo describe la JDRF. En ensayos clínicos, los sistemas PLGS demostraron una reducción en la hipoglucemia nocturna de un $50 \%$ en comparación con la terapia SAP $\sin$ producir cetosis matutina en los sujetos 36, 188 194.

El primer producto comercial para el control híbrido de la glucosa se lanzó al mercado en EE.UU. (sistema MiniMed 670G@, Medtronic Inc., Northridge CA) a finales de 2016. Una de sus principales características es la suspensión total del suministro de insulina cuando el sensor de glucosa cae por debajo de un valor o se predice una hipoglucemia, gracias al combo CGM-CSII [195]. El sistema PLGS predice el valor de glucosa basándose en un predictor de Holt modificado. El umbral hipoglucémico y el horizonte de predicción son parámetros ajustables. El umbral hipoglucémico es la lectura de glucosa predicha por debajo de la cual ocurre la suspensión de la bomba, y el horizonte de predicción es el tiempo que toma alcanzar el umbral hipoglucémico. El dispositivo suspende automáticamente la infusión de insulina basal si la diferencia entre la glucosa actual del sensor y el umbral hipoglucémico es inferior a un valor determinado y se ha predicho que la glucosa del sensor será inferior al umbral en 30 minutos 186. Otro dispositivos, como la bomba de insulina Tandem t:slim X2 ${ }^{\mathrm{TM}}$ se ha utilizado con el sensor Dexcom G5 para implementar un sistema PLGS, obteniéndose una reducción del $31 \%$ del tiempo de la glucosa por debajo de $70 \mathrm{mg} / \mathrm{dl}$, en comparación con la terapia SAP 196. Este sistema PLGS, patentado como Basal-IQ ${ }^{\mathrm{TM}}$, suspende la administración de insulina en cualquiera de los siguientes casos: si la concentración de glucosa medida cae por debajo de $70 \mathrm{mg} / \mathrm{dL}$; o si la glucosa prevista en 30 minutos está por debajo de un cierto umbral. La administración de insulina se reanuda cuando el sistema recibe una lectura de glucosa más alta que la lectura anterior. Este sistema es similar al de Medtronic, y ambos representan una mejora respecto al corte mediante un cierto umbral de los sistemas LGS 197.

Naturalmente, el rendimiento de los sistemas PLGS con un enfoque basado en el modelo del sistema glucosa-insulina, dependen de la eficacia de la predicción para un individuo determinado. En los últimos años se han propuesto varias estrategias para predecir la glucosa en plasma a partir de modelos matemáticos 198. La mayoría de estos estimadores son de alto orden y no-lineales 199 y una individualización de estos modelos conlleva una gran dificultad [55]. Un enfoque interesante para abordad esta dificultad es la de estimadores basados en datos (data-driven). Por ejemplo, en [200] se implementan modelos basados en series temporales y se integran con métodos de identificación recursiva, que permite la adaptación dinámica del modelo a la variabilidad inter-/intra-paciente y a las perturbaciones glucémicas. Esta metodología no representa problemas de aproximación y puede afrontar eficazmente sistemas no-lineales. Los algoritmos de control basados en datos también muestran un buen rendimiento en conjunción con los estimadores basados en datos, como se presenta en [201].

\subsection{Algoritmo GSAFE}

El algoritmo que se propone consiste en dos elementos : (1) la predicción de la glucosa en plasma mediante un Predictor basado en Filtro de Kalman (Kalman Filter Predictor $(\underline{\mathrm{KFP}})$ ); (2) una estrategia de lazo cerrado basada en la técnica de SMRC propuesta en trabajos anteriores de los autores 162 que busca limitar la tasa de decrecimiento de la glucosa predicha para que no sea inferior a un determinado umbral preestablecido. 


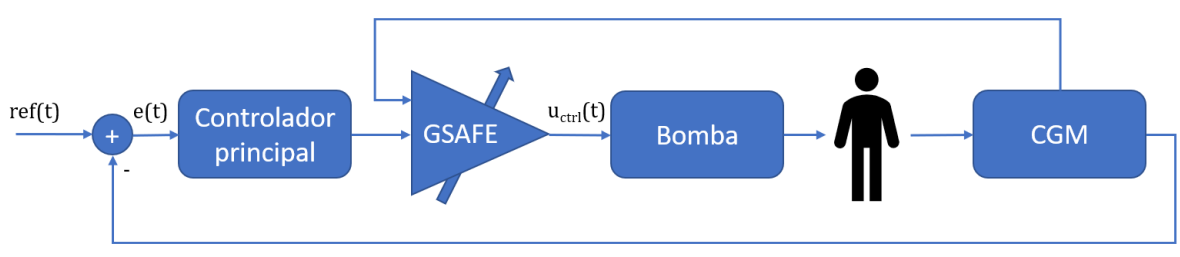

Figura 5.1: Diagrama de bloques de la estructura de control propuesta.

A diferencia de los trabajos anteriores donde la limitación se estableció en los primeros estados de la dinámica de la insulina conocida como IOB [20,141, 154], la metodología propuesta en este capítulo pretende, desde el punto de vista de la teoría de control, imponer una restricción a la variable de salida del sistema. En este caso, la administración de insulina no se detiene durante cierto tiempo sino que se reanuda gradualmente, reduciendo así el riesgo de hipoglucemia y permitiendo también que la insulina esté disponible antes en caso de futuros rebotes o de hiperglucemia.

En la Figura 5.1 puede verse un diagrama de bloques de la propuesta. Consiste en el CGM, un controlador principal y la capa GSAFE. El algoritmo de GSAFE se basa en una predicción de GP por un KFP y un algoritmo adaptativo Sliding-Mode (SM). La capa actúa como una ganancia adaptativa para el controlador de realimentación, que depende de la tasa de decaimiento de la GP predicha.

\subsubsection{Predictor de Filtro de Kalman}

Es de interés predecir la evolución de la GP para prevenir un evento hipoglucémico. También es importante que la glucosa predicha sea fiable, por lo que es necesario un predictor de lazo cerrado para descartar predicciones alejadas de la realidad. En este caso, la predicción de la glucosa será realizada por un KFP, que se presenta en 202. Para describir cómo se realiza la predicción, también se introduce el Kalman Filter (KF).

Tanto la formulación del KF como de KFP se basan en un proceso dado en el espacio de estados (ec. (5.1)), donde se asume una entrada $u$ y una salida $y$, con un ruido de proceso $w$ y un ruido de medición $v$ :

$$
\begin{aligned}
x(k+1) & =\Phi_{1} x(k)+\Lambda_{1} u(k)+\Gamma_{1} w(k) \\
y(k) & =\Theta_{1} x(k)+v(k) .
\end{aligned}
$$

Las matrices $\Phi_{1} \in \mathcal{R}^{n \times n}, \Lambda_{1} \in \mathcal{R}^{n \times 1}, \Gamma_{1} \in \mathcal{R}^{n \times 1}, \Theta_{1} \in \mathcal{R}^{1 \times n}$ reprentan el sistema discreto, $x(k)$ es un vector de estados y $w(k)$ y $v(k)$ se asumen como secuencias no correlacionadas con media cero. Las matrices de covarianza de ruido se definen como:

$$
\begin{aligned}
E\left\{w(k) w(k)^{T}\right\} & =R_{w} \\
E\left\{v(k) v(k)^{T}\right\} & =R_{v} .
\end{aligned}
$$

para el proceso (5.1), el KF es un estimador óptimo de los estados $x(k)$ 203]. El algoritmo $\mathrm{KF}$ variante en el tiempo, el cual actualiza los estados $\hat{x}(k)$ y la covarianza de los mismos $\hat{P}(k)$, se describe de la siguiente forma:

a) Cálculo de la ganancia:

$$
K(k)=\hat{P}^{-}(k) \Theta_{1}^{T}\left[\Theta_{1} \hat{P}^{-}(k) \Theta_{1}^{T}+R_{v}\right]^{-1}
$$

b) Actualización de medida (cuando llega una medida):

$$
\begin{gathered}
\hat{x}^{+}(k)=\hat{x}^{-}(k)+K(k)\left[y(k)-\Theta_{1} \hat{x}^{-}(k)\right] \\
\hat{P}^{+}(k)=\hat{P}^{-}(k)-K(k) \Phi_{1} \hat{P}^{-}(k)
\end{gathered}
$$




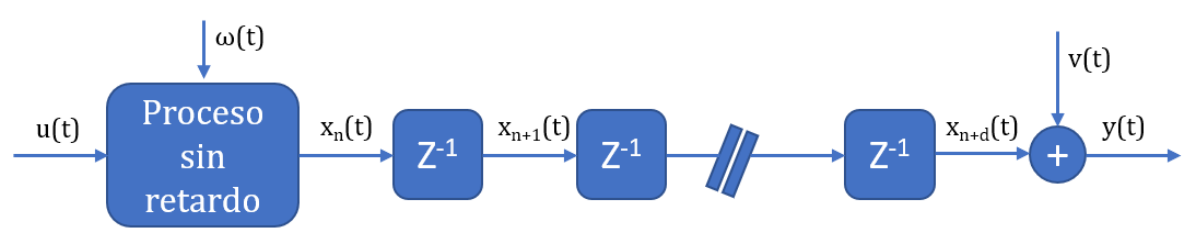

Figura 5.2: Proceso SISO con estados expandidos para representar el retardo $d$.

c) Actualización en el tiempo (entre mediciones):

$$
\begin{gathered}
\hat{x}^{-}(k+1)=\Phi_{1} \hat{x}^{+}(k)+\Lambda_{1} u(k) \\
\hat{P}^{-}(k+1)=\Phi_{1} P^{-}(k) \Phi_{1}^{T}+\Gamma_{1} R_{w} \Gamma_{1}
\end{gathered}
$$

Cuando hay un retardo en la salida del proceso (por ejemplo dado por $e^{-d s}$ en la forma de Laplace), el esquema que puede utilizarse para la predicción es el del KFP. Por un lado, el proceso se define sin el retardo, tal como se lo hizo en (5.1), y por otro, el retardo del sistema se expresa considerando una cantidad $d$ de retardos de primer orden a la salida del proceso. Si se asume que el retardo del proceso es un numero entero conocido múltiplo de la tasa de muestreo del sistema discreto, el procesos con retardo puede ser representado mediante los siguiente estados expandidos:

$$
x_{i}(k+1)=x_{i-1}(k) \quad i=n+1, \ldots, n+d .
$$

La interpretación en diagrama de bloques de todo el proceso expandido se representa en la Fig. 5.2.

Se presupone que sólo los estados del proceso, sin retardos de tiempo, están influenciados por el ruido del proceso, y que el ruido de medición se introduce a la salida del mismo, correspondiente al estado $x_{n+d}$. El sistema Single-Input-Single-Output (SISO) aumentado del proceso global puede definirse mediante las siguientes matrices de modelo de espacio de estado:

$$
\begin{array}{rlrl}
\Phi & =\left[\begin{array}{cc}
\Phi_{1} & 0 \\
\Phi_{x} & \Phi_{2}
\end{array}\right]_{(n+d) \times(n+d)} & \Lambda=\left[\begin{array}{c}
\Lambda_{1} \\
0
\end{array}\right]_{(n+d) \times 1} \\
\Gamma & =\left[\begin{array}{c}
\Gamma_{1} \\
0
\end{array}\right]_{(n+d) \times 1} \quad \Theta=\left[\begin{array}{ll}
0 & \Theta_{2}
\end{array}\right]_{1 \times(n+d)}
\end{array}
$$

with

$$
\begin{aligned}
\Phi_{2} & =\left[\begin{array}{ccccc}
0 & 0 & \cdots & 0 & 0 \\
1 & 0 & \cdots & 0 & 0 \\
0 & 1 & \cdots & 0 & 0 \\
\vdots & \vdots & & \vdots & \vdots \\
0 & 0 & \cdots & 1 & 0
\end{array}\right]_{d \times d} \quad \Phi_{x}=\left[\begin{array}{c}
\Theta_{1} \\
0
\end{array}\right]_{d \times n} \\
\Theta_{2} & =\left[\begin{array}{lllll}
0 & 0 & \cdots & 0 & 1
\end{array}\right]_{1 \times d} .
\end{aligned}
$$

Cabe señalar que el KFP tal como se definió, tiene una estructura similar al predictor de Smith, con la diferencia de que incluye un filtro óptimo para aplicaciones de procesos estocásticos con ruido gaussiano.

El predictor propuesto no está diseñado para funcionar como en la mayoría de los sistemas de PLGS, es decir, para estimar los valores de glucosa en un horizonte de predicción con el fin de predecir una hipoglucemia y detener el suministro de insulina. La intención del predictor en este caso consiste en predecir el valor de la pendiente de la glucosa hasta la siguiente muestra mediante medidas de CGM y la dinámica del modelo de glucosainsulina. El KFP se utilizará para predecir la $\overline{G P}(\hat{G})$ y su primera derivada $(\hat{\dot{G}})$ para luego ajustar la ganancia del controlador como se describe en la siguiente sección. 


\subsubsection{Adaptación de ganancia}

La propuesta tiene como objetivo limitar la pendiente con la que la glucosa disminuye. Para ello, se debe encontrar la cantidad de insulina que debe suministrar el controlador que cumpla con la condición impuesta. El cálculo analítico de esta entrada es engorroso y complejo de llevar a cabo de manera a priori. En su lugar, se propone el uso de una ganancia adaptativa aplicada a la salida de un controlador principal. El cálculo de esta ganancia se puede realizar aplicando la teoría de $\underline{\mathrm{SM}}$. Este método es robusto, no requiere de un cálculo de dinámica inversa y puede ser aplicado a cualquier tipo de controlador. En este caso, la metodología utilizada se basa en la técnica SMRC desarrollada por los directores y colegas [204, y que se combinó con los controladores PID en sus primeras aplicaciones a la regulación de la glucosa 141.

En cada nueva medición, el perfil de glucosa de los próximos 5 minutos será predicho por el KFP (tiempo entre muestras) para estimar la pendiente actual de la glucosa en sangre y ajustar la salida de los controladores en consecuencia. La implementación en software del predictor permite utilizar una tasa de muestreo superior al tiempo de muestreo de CGM. Para cada iteración, se estima el valor de GP y su derivada. Se establece una restricción en la derivada de la glucosa, con el objetivo de evitar que la glucosa disminuya a una tasa inferior a la impuesta. Hay que tener en cuenta que cuando se habla de "tasa inferior" es en términos de valores negativos de la derivada de la glucosa y no en términos de velocidad (valor absoluto de la derivada). En caso de que la derivada de la glucosa estimada no cumpla con la restricción, la capa de GSAFE adaptará la infusión de insulina dada por un controlador principal mediante una ganancia adaptativa variable en el tiempo $\gamma$, que puede tomar valores entre 0 y 1 . De esta manera, la dosis de insulina es regulada por la ganancia adaptativa $\gamma$ en lugar de suspender totalmente la administración de insulina como lo hacen generalmente los sistemas PLGS.

Para limitar la tasa de decrecimiento de la glucosa, se propone una ley de conmutación de la siguiente manera:

$$
\omega(t)=\left\{\begin{array}{lll}
1 & \text { if } & \sigma(t)>0 \\
0 & \text { if } & \sigma(t) \leq 0
\end{array}\right.
$$

donde, suponiendo un controlador bipropio sin perder generalidad, la función de conmutación $\sigma(t)$ se diseña como

$$
\sigma(t)=\hat{\dot{G}}(t)-\overline{\dot{G}},
$$

para imponer una restricción en la tasa de decrecimiento en la predicción de GP i.e. $\hat{\dot{G}}(t) \geq \stackrel{\bar{G}}{\text {. }}$

$\mathrm{El}$ algoritmo propuesto funciona de la siguiente manera. Después de que se ingiere una comida, la GP se eleva, y también se incrementa la infusión de insulina por el controlador. Luego, la insulina suministrada comienza a actuar sobre la GP, por lo que comienza a disminuir el pico posprandial. Cuando la pendiente con la que disminuye la glucosa es menor que el límite preestablecido $\bar{G}$ la función $\sigma(t)$ se hará negativa y $\omega(t)=0$, por lo tanto, se suspenderá el suministro de insulina. Esto se mantendrá a medida que la tasa de descrecimiento de la glucosa predicha sea inferior a la impuesta. Una vez que la insulina suministrada por el controlador para contrarrestar el pico posprandial sea absorbida y la derivada de la glucosa estimada $\dot{\hat{G}}(t)$ alcance $\overline{\dot{G}}$, una conmutación de alta frecuencia de $\omega(t)$ entre 1 y 0 ocurrirá (modo deslizante). Los valores de la señal $\omega$ son guardados en un vector y luego promediados, obteniéndose así $\gamma$. La ganancia $\gamma$ resultará en un valor entre 0 y 1 que escalará la salida del controlador hasta que su acción ya no cause la violación de la restricción.

Se desea que por encima de $\overline{\dot{G}}$ el controlador actúe libremente, esto implica $\omega(t)=1$ para $\sigma(t)>0$. Cuando la restricción impuesta es violada $(\sigma(t)<0)$, el efecto de $\omega(t)=0$ 
buscará que se restaure $\dot{\hat{G}}>\overline{\dot{G}}$ y $\dot{\sigma}(t)>0$. Para cumplir con la condición necesaria para que se establezca un régimen de modo deslizante, el grado relativo entre $\sigma(t)$ y la señal discontinua $\omega(t)$ debe ser igual a la unidad, esto significa que $\omega(t)$ aparezca explícitamente en la primera derivada de $\sigma(t)$. Por lo tanto, el diseño del predictor está sujeto a esta restricción, implicando que el modelo utilizado sea de orden reducido.

\subsubsection{Implementación de la predicción de GP}

Debido a la restricción de diseño impuesta por la ganancia adaptiva obtenida mediante modos deslizantes, se opta por un modelo de orden reducido del sistema glucosa-insulina. Se considera implementar el modelo presentado en el Capítulo 2 sección 2.3. Este modelo LPV [159 fue obtenido a partir del simulador UVA/Padova 75] y su desempeño fue comparado con otros presentados en la literatura [67, 105]. El modelo de bajo orden identificado se representa por la siguiente función de transferencia:

$$
\frac{G(s)}{U(s)}=k \frac{(s+z)}{\left(s+p_{1}\right)\left(s+p_{2}\right)\left(s+p_{3}\right)} e^{-15 s}
$$

donde la entrada corresponde al suministro de insulina subcutánea $[\mathrm{pmol} / \mathrm{kg}$ y la salida a la concentración de glucosa $[\mathrm{mg} / \mathrm{dl}]$. El modelo puede ser individualizado a partir de la ganancia $k$ usando la regla ad-hoc de 1800. Para la simplificación del control, se utiliza una implementación LTI de este modelo, como en trabajos anteriores 20]. La obtención del modelo LTI se realiza a partir de la selección de un punto de operación $g_{b}$ mantenido por un suministro basal $i_{b}$, obteniéndose el polo dominante $p_{1}\left(g_{b}\right)$ [159].

A partir de la representación en Laplace del modelo (5.12), se realiza la realización en espacio de estados a partir de la forma canónica observable:

$$
\begin{aligned}
\dot{x} & =A x(t)+B u_{\Delta}(t) \\
y_{\Delta} & =C x(t),
\end{aligned}
$$

con

$$
A=\left[\begin{array}{ccc}
0 & 1 & 0 \\
0 & 0 & 1 \\
-\alpha_{1} & -\alpha_{2} & -\alpha_{3}
\end{array}\right], B=\left[\begin{array}{c}
k z \\
k \\
0
\end{array}\right], C=\left[\begin{array}{lll}
1 & 0 & 0
\end{array}\right],
$$

donde $x(t)$ son los estados del sistema glucosa-insulina, $u_{\Delta}(t)$ y $y_{\Delta}$ son las desviaciones del punto de operación $\left(u_{o p} ; y_{o p}\right)$ y $\alpha_{i=1,2,3}$, corresponden a los coeficientes del polinomio característico del sistema (5.12), siendo $\alpha_{1}=p_{1} p_{2} p_{3}, \alpha_{2}=\left(p_{2} p_{3}+p_{1} p_{2}+p_{1} p_{3}\right), \alpha_{3}=\left(p_{1}+\right.$ $\left.p_{2}+p_{3}\right)$ respectivamente. Cabe señalar que el estado $x_{1}(t)$ corresponde a la concentración de glucosa y $x_{2}(t)$ a su derivada, mientras que el retardo aún no es considerado.

El modelo (5.13) se discretiza para obtener las matrices $\Phi_{1}, \Theta_{1}$ y $\Lambda_{1}$ (correspondientes al proceso (5.1)) y así ser implementado en la forma de un KFP. El tiempo de muestreo de los sensores CGM (1-10min) es significativamente menor que las dinámicas del sistema glucosa-insulina (100min aproximadamente), por lo que se pueden asumir entradas constantes a trozos durante el tiempo de discretización $\Delta t$ sin perder exactitud. Al expandir el sistema considerando el retardo de tiempo, el grado relativo igual a 2 entre la entrada $u_{\Delta}(t)$ y la salida $y_{\Delta}=x_{1}(t)$ (GP estimada $\hat{G}$ ) del modelo 5.13 se mantiene, pero el sistema global se expande a un orden igual a $n+d$.

El KFP pondera las incertidumbres del modelo dinámico de la glucosa y el ruido de las medidas de CGM para realizar la estimación. La incertidumbre que contribuye la variabilidad inter- e intra-paciente puede ser interpretada como ruido del proceso y ser considerada mediante la sintonización de la matriz de covarianza $R_{w}$. Además, las perturbaciones como las comidas pueden ser representadas como ruido en la primera derivada de la glucosa estimada, ya que el predictor se propone sin el anuncio de las mismas (lazo cerrado puro). 
La predicción de glucosa propuesta usando el modelo (5.13) tiene cierta similitud con el algoritmo presentado en 205] con la excepción de que aquí también se tiene en cuenta la acción de la insulina y el retraso del sistema en el modelo del predictor de glucosa. En este enfoque, las variaciones del proceso y el ruido de la observación se han adaptado heurísticamente considerando 205 sintonizando $R_{w}=\operatorname{diag}(0 ; 0,1 ; 0,01)$ y $R_{v}=8 \mathrm{mg} / \mathrm{dl}$, respectivamente.

\subsection{Ejemplos de aplicación del GSAFE}

En cuanto a la aplicabilidad de la propuesta, ya se ha mencionado que se puede utilizar cualquier controlador principal. En particular, cabe destacar la viabilidad de aplicar la capa GSAFE a las técnicas de control ampliamente difundiadas en los sitemas de Páncreas Artificial. Los siguientes ejemplos de aplicación se realizan siguiendo la estructura de control presentado en la Fig. 5.1.

\subsubsection{Controlador MPC}

Se implementa un controlador lineal estándar MPC basado en el trabajo presentado en [206]. Se ejecuta en tiempo discreto y calcula una ley de control numérico cada 5 minutos después de la llegada de una medición de glucosa. La corrección de la dosis de la infusión de insulina a partir de la tasa basal $u_{k}$, se obtiene resolviendo un problema de optimización de una función objetiva definida como

$$
J(\cdot)=\sum_{k=0}^{N-1}\left[\mathcal{Q} \cdot\left(y_{r e f}-y_{k}\right)^{2}+\mathcal{R} \cdot u_{k}^{2}\right]+x_{N}^{T} P x_{N},
$$

donde $y_{k}(\mathrm{mg} / \mathrm{dl})$ indica las predicciones de glucosa a partir del valor de glucosa basal, $u_{k}(\mathrm{pmol} / \mathrm{kg})$ indica la corrección de insulina respecto al suministro basal y $y_{\text {ref }}$ es el valor de glucosa de referencia. $\mathcal{Q}$ y $\mathcal{R}$ son pesos escalares positivos y $N$ es el horizonte de predicción. El peso asociado al error de glucosa $\mathcal{Q}$ se sintonizó de acuerdo a la sensibilidad insulínica del paciente a partir de su TDI. El peso en la acción de control $\mathcal{R}$ se ajustó de acuerdo al rango etario. La matriz $P$ corresponde a la solución única no-negativa de la ecuación algebraica discreta de Riccati

$$
A^{T} P A-P-A^{T} P B\left(B^{T} P B+R\right)^{-1} B^{T} P A=0 .
$$

Se consideraron las restricciones de entrada debido a la saturación de la bomba de insulina. Finalmente, se cuantifica la insulina total para que sea infundida por la bomba de insulina.

El modelo utilizado en la estructura del controlador MPC para la predicción de los estados $x_{i}$ corresponde al modelo glucosa-insulina LTI dado en la Sección 2.3. Se ajustan los valores de los parámetros $p_{1}$ y $k$ de acuerdo al punto de operación dado por $y_{\text {ref }}$. Como observador de estados se aplica el KFP de acuerdo a la Sección 5.2.1. Nótese que el controlador MPC presenta la ventaja de tener una predicción de glucosa basada en modelo en su estructura interna, por lo tanto simplifica la implementación de la capa GSAFE y proporciona un potencial aún mayor a la propuesta.

A fin de ilustrar la acción de la capa GSAFE sobre el controlador MPC, se propone un escenario posprandial para un sujeto adolescente virtual del simulador de UVA/Padova. Se administró una comida de $60 \mathrm{~g}$ en $\mathrm{t}=420$ minutos y se hace una comparación del funcionamiento del controlador principal con y sin la capa. En ambos casos, el tiempo de muestreo del algoritmo GSAFE fue de 0,5min y el tiempo de muestreo del controlador fue de 5 min. Se estableció una restricción en la tasa de decrecimiento de la glucosa igual a 

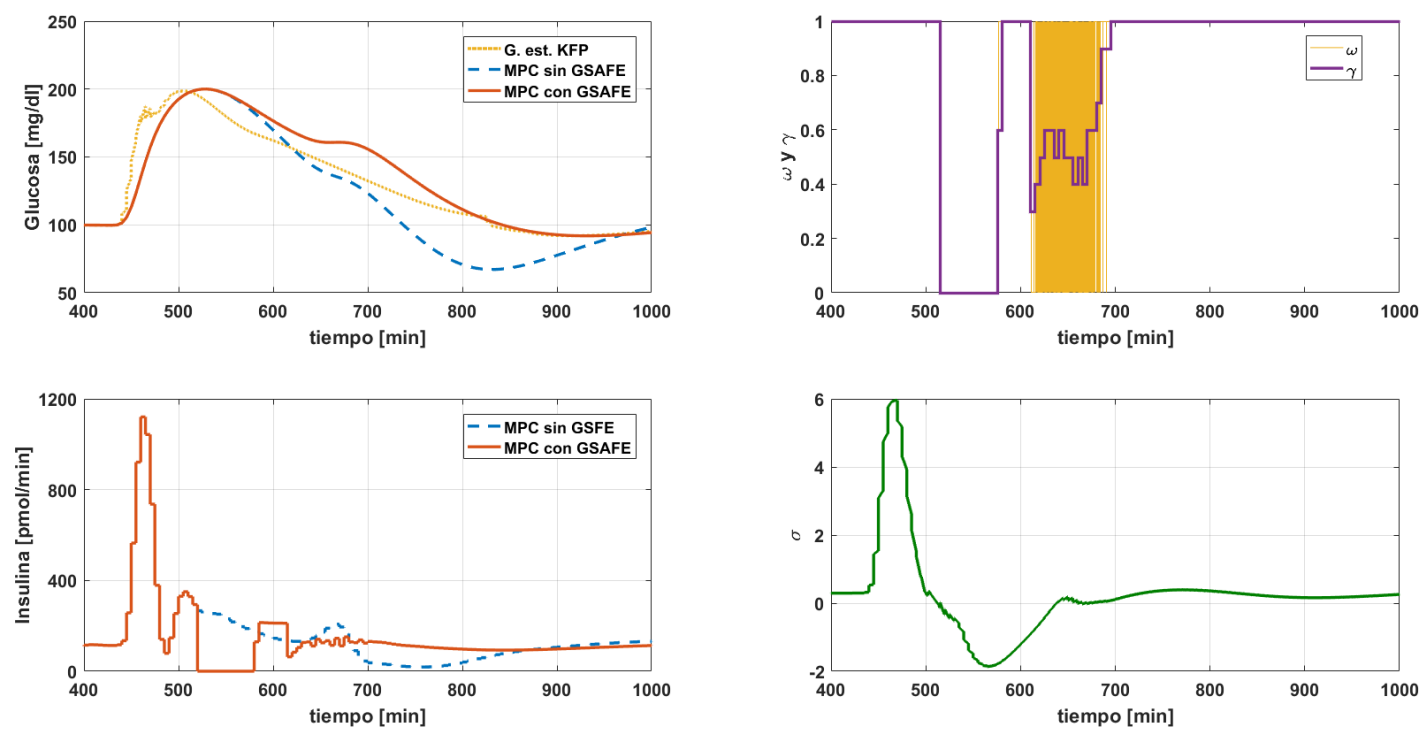

Figura 5.3: Ejemplo ilustrativo de la capa de GSAFE sobre un controlador MPC en un sujeto virtual. En la esquina superior izquierda se observa la excursión de la glucosa resultante por controlador sin (línea punteada azul) y con la capa GSAFE (línea sólida naranja), la glucosa predicha por el KFP (línea punteada amarilla) y el umbral de hipoglucemia. En la esquina inferior izquierda, se observa la insulina administrada por el controlador sin (línea punteada azul) y con la capa GSAFE (línea naranja continua). A la derecha, las señales $\gamma$ y $\omega$ (arriba) y $\sigma$ (abajo) de la capa GSAFE.

$\overline{\dot{G}}=-0,3 \mathrm{mg} / \mathrm{dl} / \mathrm{min}$. El controlador principal se sintonizó de acuerdo a la descripción en la Sección 5.3 .1 .

En la Fig. 5.3 se muestran las señales resultantes. En la esquina superior izquierda se muestra la comparación entre la excursión de la glucosa (CGM) del sujeto resultante del controlador MPC (línea azul punteada) y del MPC+GSAFE (línea naranja continua). También se muestra la glucosa pronosticada por el KFP para este último caso (línea punteada amarilla). En la esquina inferior izquierda de la figura, se muestra la insulina administrada por el MPC (línea punteada azul) y el MPC+GSAFE (línea naranja continua). Las señales $\gamma$ y $\omega$ (arriba) y $\sigma$ (abajo) de la capa GSAFE se muestran en la mitad derecha de la figura.

Puede verse en la Fig. 5.3 que las medidas de CGM empiezan a crecer después de la ingesta de la comida. La predicción de la GP (primer estado del sistema KFP) se actualiza de acuerdo al modelo utilizado y de acuerdo a las matrices de covarianza. Después del pico post-prandial, la glucosa comienza a disminuir y la derivada predicha de glucosa cae por debajo del umbral $(\sigma(t)=0)$, por lo que la señal $\omega$ cambia a cero $(\mathrm{t}=515 \mathrm{~min})$. Debido a que todavía hay insulina actuando sobre la GP. no es posible limitar su tasa de disminución, por lo que $\omega$ permanece igual a cero. Por lo tanto, la ganancia adaptativa $\gamma$ también será cero, suspendiendo la infusión de insulina. En t $=580 \mathrm{~min}$, la glucosa estimada coincide con la restricción y la capa GSAFE permite al controlador actuar libremente sin exceder la restricción. Cuando la insulina administrada comienza a actuar en el cuerpo, por lo que la pendiente de la glucosa alcanza de nuevo la restricción, se produce una conmutación de $\omega$. Se puede ver cómo tanto el perfil de glucosa predicho como el perfil de glucosa de CGM se suavizan entre los 605 y 700min por la acción de la ganancia adaptativa $\gamma$. Después de ese periodo de adaptación, el controlador puede actuar libremente de nuevo y la GP vuelve suavemente a la referencia. Por el contrario, el controlador principal sin la capa de GSAFE resulta ser más agresivo. En particular, el segundo pico de absorción de las comidas causa un aumento en la infusión de insulina, que junto con la insulina todavía activa en el cuerpo conduce a un episodio hipoglucémico en $t=800 \mathrm{~min}$. 


\subsubsection{Controlador PDBasal+IFB}

En base al clásico algoritmo de control PID se plantea el controlador PDBasal+IFB [165. En primer lugar, el término integral se sustituye por la insulina basal de la terapia de lazo abierto. De este forma, se elimina una posible sobreactuación debido al término integral del controlador y el efecto windup. Por lo tanto, la salida del controlador PDBasal se define como:

$$
u_{p d b}(t)=k_{p}\left[e(t)+\tau_{d} \frac{d e(t)}{d t}\right]+u_{b a s a l}(t)
$$

donde la ganancia proporcional $k_{p}$ se define como

$$
k_{p}=\frac{60}{\tau_{d}} \frac{I_{T D I}}{1500},
$$

siendo $I_{T D I}$ la insulina total diaria $[166] ; \tau_{d}(t)$ posee un valor nominal de $90 \mathrm{~min}$ durante el día y 60min durante la noche $165 ; e(t)=C G M-r e f$ donde CGM es la medición de la glucosa y ref el valor de referencia de glucosa; y $u_{\text {basal }}(t)$ corresponde al perfil de insulina basal del sujeto.

Además de la estructura PD, se introduce el término IFB para reproducir la respuesta de las células $\beta$ ante la concentración de insulina en plasma (PIC). La secreción de insulina de las células beta se inhibe a medida que aumenta la PIC. Para poder implementar el término IFB, el PIC debe ser estimado de manera on-line a partir de la insulina infundida. Se asume un modelo de dos compartimentos para la farmacocinética de la insulina, dado por el siguiente sistema:

$$
\begin{aligned}
\dot{L}(t) & =-\frac{1}{\tau_{2}} L(t)+u_{c t r l}(t) \\
\dot{I}_{p}(t) & =\frac{1}{\tau_{1} \tau_{2} K_{C L}} L(t)-\frac{1}{\tau_{1}} I_{p}(t),
\end{aligned}
$$

donde $u_{c t r l}$ es la entrada de insulina, $L$ es el compartimento intermedio e $I_{p}$ es la PIC estimada. Los valores para las constantes de tiempo del sistema son $\tau_{1}=55 \mathrm{~min}$ y $\tau_{2}=$ 70min, y para la eliminación de la insulina $K_{c l}=1 \mathrm{~mL} / \mathrm{min}$. El efecto de la PIC dado por $I_{p}(t)$ se agrega a la expresión (5.16) afectada por la ganancia $k_{I F B}$. Esta última ganancia se ajusta de acuerdo a 165 .

Finalmente, la señal que comanda la bomba de insulina esta dada por

$$
u_{c t r l}(t)=\left(u_{p d b}-k_{I F B} I_{p}(t)\right) \cdot \gamma
$$

En la Fig 5.4 puede verse la acción de la capa GSAFE sobre un controlador PDBasal+IFB (presentado en la Sección 5.3) sobre un sujeto adulto ante una comida de $100 \mathrm{~g}$ de CHO. En este caso, la comida es de mayor tamaño que en el caso anterior. Esto produce que la acción del controlador sin la capa GSAFE sea igua a 0 debido a la acción derivativa. La acción de la capa GSAFE es análoga a la descripta sobre el controlador MPC y puede observarse en este caso cómo, gracias a la predicción de GP la cual tiene en cuenta el retardo del sistema, se suspende antes el suministro de insulina (respecto al controlador sin la capa) y luego se reanuda suavemente mediante la adaptación de la ganancia $\gamma$ para luego dejar actuar libremente al controlador.

En este ejemplo, se puede ver claramente cómo la salida del controlador se adapta mediante la ganancia variante en el tiempo $\gamma$ para evitar que la pendiente de la glucosa sea menor que la restricción impuesta. Es importante notar que esta técnica permite regular la dosificación de insulina para evitar episodios hipoglucémicos pero al mismo tiempo, permite disponer de más insulina, en comparación con la suspensión total de insulina, para hacer frente a futuras ingestas o para evitar un rebote en la glucosa. 

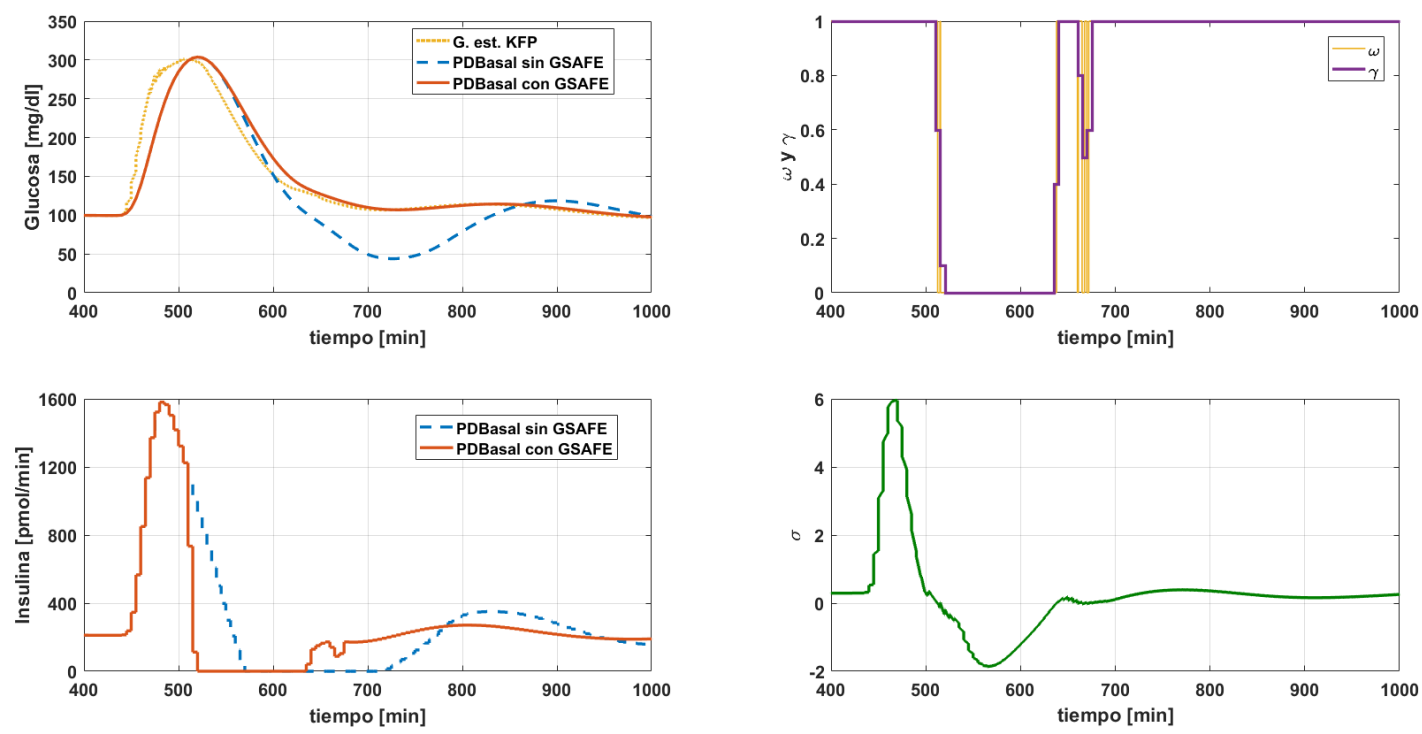

Figura 5.4: Ejemplo ilustrativo de la capa de GSAFE sobre un controlador PDBasal+IFB en un sujeto virtual. En la esquina superior izquierda se observa la excursión de la glucosa resultante por controlador sin (línea punteada azul) y con la capa GSAFE (línea sólida naranja), la glucosa predicha por el KFP (línea punteada amarilla) y el umbral de hipoglucemia. En la esquina inferior izquierda, se observa la insulina administrada por el controlador sin (línea punteada azul) y con la capa GSAFE (línea naranja continua). A la derecha, las señales $\gamma$ y $\omega$ (arriba) y $\sigma$ (abajo) de la capa GSAFE.

\subsection{Resultados in-silico con diferentes controladores}

Con el fin de evaluar el rendimiento del algoritmo propuesto, una serie de ensayos in-silico fueron realizados en la plataforma de simulación UNLP. Se propusieron dos escenarios de un solo día para una cohorte de sujetos de $n=33$, incluyendo niños, adolescentes y adultos. Se consideraron tres comidas, a las 7, 13 y 21 horas de 75, 40 y 60 gramos de CHO respectivamente, en una simulación de 24 horas de duración. Un escenario (Escenario 1) considera los parámetros nominales de los sujetos virtuales mientras que el otro considera variación intra-paciente (Escenario 2), de acuerdo a lo descripto en la Sección 2.2 .2 del Capítulo 2.

Se consideraron 3 estructuras de control para realizar la comparación en ambos escenarios propuestos:

- En primer lugar, el controlador MPC presentado anteriormente en la sección 5.3 se evaluó con y sin la capa GSAFE. Dado que el objetivo de este estudio es mostrar la capacidad de la capa GSAFE para corregir los eventos hipoglucémicos dados por una estructura de control de lazo cerrado puro, se utiliza una sintonización genérica del controlador de acuerdo al rango etario.

- En segundo lugar, se implementa el controlador PDBasal+IFB presentado en la sección 5.3 y se compara su desempeño con y sin la capa GSAFE.

- Por último, se consideró una estructura PID con un bolo fijo en el momento del anuncio de la comida, tal como se describe en el trabajo [141], con el fin de evaluar el rendimiento de la capa GSAFE en contraste con la capa SAFE. Es importante señalar que el algoritmo SAFE requiere de un anuncio de comida para poder modificar la restricción $\amalg \mathrm{IOB}$ durante el periodo posprandial. Al contrario, la capa GSAFE no utiliza esta información.

Los porcentajes resultantes de tiempo en permanencia en rango (TIR: GP $\in[70,180]$ $\mathrm{mg} / \mathrm{dl})$, debajo del rango $\left(\mathrm{TBR}_{1}: \mathrm{GP}<70 \mathrm{mg} / \mathrm{dl}\right.$ y $\left.\mathrm{TBR}_{2}: \mathrm{GP}<54 \mathrm{mg} / \mathrm{dl}\right)$ y por encima 
Tabla 5.1: Resultados poblacionales: controlador MPC

\begin{tabular}{|c|c|c|c|c|c|c|c|}
\hline & $\begin{array}{l}\text { GP Media } \\
(\mathrm{mg} / \mathrm{dl})\end{array}$ & $\begin{array}{c}\text { Variabilidad GP } \\
(\% \mathrm{CV})\end{array}$ & $\begin{array}{c}\mathrm{GP}<54 \mathrm{mg} / \mathrm{dl} \\
(\% \text { tiempo })\end{array}$ & $\begin{array}{c}\mathrm{GP}<70 \mathrm{mg} / \mathrm{dl} \\
(\% \text { tiempo })\end{array}$ & $\begin{array}{c}\mathrm{GP} \in[70,180] \mathrm{mg} / \mathrm{dl} \\
(\% \text { tiempo })\end{array}$ & $\begin{array}{c}\mathrm{GP}>180 \mathrm{mg} / \mathrm{dl} \\
(\% \text { tiempo })\end{array}$ & $\begin{array}{c}\mathrm{GP}>250 \mathrm{mg} / \mathrm{dl} \\
(\% \text { tiempo })\end{array}$ \\
\hline \multicolumn{8}{|c|}{ Escenario 1 - nominal } \\
\hline Con GSAFE & $146,38(47,69)$ & 33 & $1,24(3,4)$ & $2,28(4,86)$ & $74,27(8,79)$ & $23,45(7,73)$ & $5,68(5,7)$ \\
\hline Sin GSAFE & $132,34(55,5)$ & 42 & $3,55(5,41)$ & $7,67(8,42)$ & $72,25(12,07)$ & $20,08(6,15)$ & $5,89(5,83)$ \\
\hline$\rho$ - valor & $<0,01^{\star}$ & $<0,01^{\star}$ & $<0,01^{\star}$ & $<0,01^{\star}$ & 0,183 & $<0,01^{\star}$ & 0,081 \\
\hline \multicolumn{8}{|c|}{ Escenario 2 - variabilidad } \\
\hline Con GSAFE & $154,28(48,55)$ & 31 & $1,05(2,7)$ & $1,92(3,98)$ & $69,26(9,9)$ & $28,82(9,85)$ & $6,82(6,67)$ \\
\hline Sin GSAFE & $135,99(57,36)$ & 42 & $5,63(13,31)$ & $8,7(14)$ & $68,14(15,88)$ & $23,17(7,78)$ & $6,87(6,31)$ \\
\hline$\rho$ - valor & $<0,01^{\star}$ & $<0,01^{\star}$ & $<0,01^{\star}$ & $<0,01^{\star}$ & 0,786 & $<0,01^{\star}$ & 0,666 \\
\hline
\end{tabular}

Tabla 5.2: Resultados poblacionales: controlador PDBasal+IFB

\begin{tabular}{|c|c|c|c|c|c|c|c|}
\hline & $\begin{array}{l}\text { GP Media } \\
(\mathrm{mg} / \mathrm{dl})\end{array}$ & $\begin{array}{c}\text { Variabilidad GP } \\
(\% \mathrm{CV})\end{array}$ & $\begin{array}{c}\mathrm{GP}<54 \mathrm{mg} / \mathrm{dl} \\
(\% \text { tiempo })\end{array}$ & $\begin{array}{c}\mathrm{GP}<70 \mathrm{mg} / \mathrm{dl} \\
(\% \text { tiempo })\end{array}$ & $\begin{array}{c}\mathrm{GP} \in[70,180] \mathrm{mg} / \mathrm{dl} \\
(\% \text { tiempo })\end{array}$ & $\begin{array}{c}\text { GP }>180 \mathrm{mg} / \mathrm{dl} \\
(\% \text { tiempo })\end{array}$ & $\begin{array}{c}\text { GP }>250 \mathrm{mg} / \mathrm{dl} \\
\quad(\% \text { tiempo })\end{array}$ \\
\hline \multicolumn{8}{|c|}{ Escenario 1 - nominal } \\
\hline Con GSAFE & $147,94(43,35)$ & 29 & $0,47(2,34)$ & $0,54(2,98)$ & $76,43(11,15)$ & $23,03(10,97)$ & $5,63(7,06)$ \\
\hline Sin GSAFE & $138,25(46,51)$ & 33 & $0,85(2,71)$ & $1,81(5,07)$ & $77,11(12,35)$ & $21,08(10,57)$ & $4,63(5,83)$ \\
\hline$\rho$ - valor & $<0,01^{\star}$ & $<0,01^{\star}$ & 0,25 & $0,048^{\star}$ & $0,011^{\star}$ & $<0,01^{\star}$ & $<0,01^{\star}$ \\
\hline \multicolumn{8}{|c|}{ Escenario 2 - variabilidad } \\
\hline Con GSAFE & $158,82(45,74)$ & 28 & $0,29(1,52)$ & $0,33(1,79)$ & $69,93(12,51)$ & $29,75(12,56)$ & $7,54(9,49)$ \\
\hline Sin GSAFE & $143,21(48,06)$ & 33 & $0,98(3,15)$ & $2,23(5,61)$ & $73,37(13,68)$ & $24,4(11,92)$ & $5,78(7,26)$ \\
\hline$\rho$ - valor & $<0,01^{\star}$ & $<0,01^{\star}$ & 0,125 & $0,008^{\star}$ & $0,006^{\star}$ & $<0,01^{\star}$ & $<0,01^{\star}$ \\
\hline
\end{tabular}

Tabla 5.3: Resultados poblacionales: SAFE vs. GSAFE

\begin{tabular}{|c|c|c|c|c|c|c|c|}
\hline & $\begin{array}{l}\text { GP Media } \\
(\mathrm{mg} / \mathrm{dl})\end{array}$ & $\begin{array}{c}\text { Variabilidad GP } \\
(\% \mathrm{CV})\end{array}$ & $\begin{array}{c}\mathrm{GP}<54 \mathrm{mg} / \mathrm{dl} \\
(\% \text { tiempo })\end{array}$ & $\begin{array}{c}\mathrm{GP}<70 \mathrm{mg} / \mathrm{dl} \\
(\% \text { tiempo) }\end{array}$ & $\begin{array}{c}\mathrm{GP} \in[70,180] \mathrm{mg} / \mathrm{dl} \\
(\% \text { tiempo })\end{array}$ & $\begin{array}{c}\mathrm{GP}>180 \mathrm{mg} / \mathrm{dl} \\
(\% \text { tiempo })\end{array}$ & $\begin{array}{c}\mathrm{GP}>250 \mathrm{mg} / \mathrm{dl} \\
(\% \text { tiempo })\end{array}$ \\
\hline \multicolumn{8}{|c|}{ Escenario 1 - nominal } \\
\hline GSAFE & $139,19(40,56)$ & 29 & $1,4(5,14)$ & $2,45(6,6)$ & $79,78(11,22)$ & $17,77(8,91)$ & $4,3(4,85)$ \\
\hline SAFE & $143,12(47,11)$ & 33 & $0,58(1,97)$ & $2,27(4,05)$ & $75,4(13,74)$ & $22,33(12,84)$ & $6,34(7,18)$ \\
\hline$\rho$ - valor & 0,832 & $<0,01^{\star}$ & 0,563 & 0,811 & $<0,01^{\star}$ & $0,022^{\star}$ & $<0,01^{\star}$ \\
\hline \multicolumn{8}{|c|}{ Escenario 2 - variabilidad } \\
\hline GSAFE & $144,95(42,57)$ & 29 & $1,31(4,26)$ & $2,5(6,3)$ & $75,85(11,07)$ & $21,64(9,08)$ & $5,26(6,2)$ \\
\hline SAFE & $159,78(49,14)$ & 31 & $1,16(2,57)$ & $2,48(4,02)$ & $69,36(18,4)$ & $28,17(17,66)$ & $11,2(15,26)$ \\
\hline$\rho$ - valor & 0,491 & $<0,01^{\star}$ & 0,95 & 0,669 & $<0,01^{\star}$ & $0,025^{\star}$ & $<0,01^{\star}$ \\
\hline
\end{tabular}

$\left(\mathrm{TAR}_{1}: \mathrm{GP}>180 \mathrm{mg} / \mathrm{dl}\right.$ y $\left.\mathrm{TAR}_{2}: \mathrm{GP}>250 \mathrm{mg} / \mathrm{dl}\right)$ para toda la población se consideraron en base al informe de consenso presentado en [207]. Las métricas de evaluación para los escenarios y controladores propuestos se presentan en tablas 5.1 5.3. Para la comparación entre los tratamientos, se realizó el test de hipótesis de Wilcoxon de Rango con Signo de 2 muestras, considerando $\rho<0,05$ como estadísticamente significativo.

Los resultados de un solo día en la tabla 5.1 muestran la ventaja proporcionada por la propuesta en esquemas de lazo cerrado puro. Por un lado, la sintonización del controlador MPC demuestra ser efectiva para toda la población cuando se enfrenta a un escenario nominal, pero aún así presenta un TBR considerable (cabe señalar que no se consideró carbohidratos de rescate). Por otro lado, el tiempo en hipoglucemia se redujo considerablemente gracias a la capa GSAFE, dentro de los márgenes considerados aceptables para la comunidad. La capa GSAFE logra reducir el $\mathrm{TBR}_{1}(2,28(4,86)$ vs. $7,67(8,42)$, $\rho<0,01)$. También se redujo sustancialmente el $\mathrm{TBR}_{2}$ a márgenes aceptables $(1,24(3,4)$ vs. $3,55(5,41), \rho<0,01)$, considerando que la población más joven presenta una gran variabilidad y un gran desafío de control. Con respecto al Escenario 2, la capa logra reducir significativamente el $\mathrm{TBR}_{1}(1,92(3,98)$ vs. $8,7(14), \rho<0,01)$. Además, la capa GSAFE proporciona una reducción importante de la variabilidad de la GP en ambos escenarios (Escenario 1: 33 vs. 42, $\rho<0,01$; Escenario 2: 31 vs. 42, $\rho<0,01$ ). Cabe señalar que algunos estudios afirman que al reducir el porcentaje de variabilidad de la GP en CV $\%<33$ se proporciona una protección adicional contra la hipoglucemia en tratamientos con insulina [207]. Resultados similares pueden observarse para el controlador PDBasal+IFB en la Tab. 5.2. El tiempo en hipoglucemia pudo reducirse considerablemente gracias a la capa 
GSAFE en ambos escenarios (Escenario 1: 0,54 $\pm 2,98$ vs. 1,81 $\pm 5,07, \rho=0,048$; Escenario 2: $0,33 \pm 1,79$ vs. $2,33 \pm 5,61, \rho=0,008)$.

Como una desventaja, el tiempo en hiperglucemia se incrementa, pero lo hace en porcentaje mucho menor (Escenario 1: 23,45 (7,73) vs. 20,08 $(6,15), \rho<0,01$; Escenario 2: $28,82(9,85)$ vs. $23,17(7,78), \rho<0,01)$. Esto se debe a que el algoritmo se centra en la seguridad contra la hipoglucemia. Cabe destacar que el $\mathrm{TAR}_{2}$ no presenta una diferencia estadísticamente relevante, ya que en principio, la capa no afecta al pico posprandial. Dado que la tasa de decrecimiento se limita, después de una excursión posprandial, la glucosa alcanza el punto referencia de forma más lenta que sin la capa de seguridad, aumentando así su valor medio en el tiempo. Si las comidas son lo suficientemente grandes, cada excursión comienza con un valor de glucosa más alto debido al tiempo de absorción de la comida anterior, elevando así el tiempo de hiperglucemia. Sin embargo, este es un resultado compartido por los sistemas LGS y se encuentra reportado en la literatura [36, 188]. Para reducir el tiempo de hiperglucemia y el valor medio de glucosa, se pueden implementar diferentes reglas para activar la capa, por ejemplo considerando decisiones heurísticas. De forma similar, un controlador principal mejorado, como por ejemplo un controlador de zona adaptable [208], mejorará los resultados.

En cuanto a los resultados obtenidos del controlador PID con la capa SAFE y la capa GSAFE, es interesante la similitud del rendimiento alcanzado a pesar de que el algoritmo SAFE utiliza la información de la comida mientras que el GSAFE no lo hace. En la tabla 5.3 puede observarse que ambos algoritmos obtienen un $\mathrm{TBR}_{1}$ reducido, presentando una diferencia marginal sin relevancia estadística (Escenario 1: 2,45 (6,6) vs. $2,27(4,05)$, $\rho=0,811$; Escenario 2: 2,5 (6,3) vs. 2,48 $(4,02), \rho=0,669)$. Cabe destacar que hay una mejora por parte del algoritmo GSAFE en TIR (Escenario 1: 79,78 (11,22) vs. 75,4 (13,74), $\rho<0,01$; Escenario 2: 75,85 (11,07) vs. 69,36 $(18,4), \rho<0,01)$ y también en $\mathrm{TAR}_{1}$ (Escenario 1: $17,77(8,91)$ vs $22,33(12,84), \rho=0,022$; Escenario 2: $21,64(9,08)$ vs. 28,17 $(17,66), \rho=0,025)$.

Existen dos grandes diferencias cualitativas entre la anterior propuesta del grupo (SAFE) y la que se hace en este capítulo (GSAFE). En primer lugar, la capa GSAFE está diseñada para imponer una restricción a la variable controlada de salida del sistema, a diferencia de la capa SAFE que establece una restricción en los primeros estados de la dinámica de la insulina. Para ello, la adaptación del controlador se realiza mediante el perfil de glucosa estimado por un KFP utilizando las mediciones del CGM en lugar de utilizar una estimación de lazo abierto de IOB. En segundo lugar, la capa SAFE se diseñó teniendo en cuenta el anuncio de la comida, ya que esto permite variar el límite de IOB. Por el contrario, la capa GSAFE está diseñada para trabajar en esquemas de lazo cerrado totalmente automatizados, apuntando a los pasos finales del diseño de sistemas de PA (recuerde la Fig. 1.12). Otras ventajas de esta nueva propuesta son su capacidad para adaptarse a los cambios de sensibilidad de la insulina, y para actuar después del pico posprandial cuando la glucosa comienza a descender. En cambio, la capa SAFE puede restringir el suministro de insulina durante la absorción de la glucosa de las comidas, lo que conduce a eventos hiperglucémicos más altos. Finalmente, un beneficio importante del diseño es que ambas propuestas no se excluyen mutuamente y pueden complementarse.

\subsection{Conclusiones}

En este último capítulo de aportes de esta tesis, se ha introducido una nueva estrategia de prevención de la hipoglucemia. Motivado por los sistemas PLGS, los cuales suspenden la administración de insulina durante un período de tiempo determinado, aquí se propone regular la dosis de insulina para evitar las altas caídas de glucosa. Mediante la limitación de la tasa de descrecimiento de la glucosa, se pueden evitar con éxito los eventos hipoglucémi- 
cos después de los períodos posprandiales y también mantener una cantidad mínima de insulina para un mejor control glucémico. De hecho, el método propuesto permite que la infusión de insulina se restablezca antes que otros sistemas, evitando así una posible hipoinsulinemia que podría resultar en un futuro rebote o hiperglucemia. La metodología propuesta puede aplicarse en principio a cualquier estructura de control y combinarse con otras estrategias. Los resultados de los ensayos in-silico sobre un controlador clásico MPC mostraron que el tiempo de permanencia en la hipoglucemia se redujo en más de un $50 \%$ para una cohorte de sujetos adultos, adolescentes y niños. Además, una comparación de rendimiento entre la capa GSAFE y la capa SAFE muestra beneficios similares en la prevención de la hipoglucemia, pero se debe tener en cuenta que la capa GSAFE no tiene la necesidad de proporcionar información sobre las comidas. Aunque el algoritmo GSAFE se presentó trabajando en los periodos posprandiales, la capa puede ser ajustada para trabajar en otras ocasiones con riesgo de hipoglucemia, como el ejercicio, caso en el que también se debe asegurar un mínimo de insulina. 


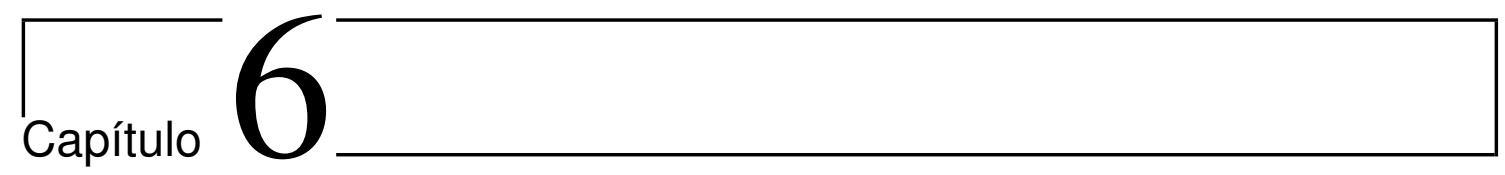

\section{Conclusiones y trabajo futuro}

En esta tesis se recorrió un camino extenso, que transcurrió por distintos rincones del universo que engloba la Diabetes Mellitus y el control de glucosa para las personas que conviven con ella. Se buscó realizar aportes en distintos aspectos del control glucémico y el tratamiento de la diabetes.

Las nuevas tecnologías desarrolladas a partir de la ruta subcutánea para el suministro de insulina y la medición de glucosa permiten el concepto del control automático de la glucosa mínimamente invasivo, nombrado como "Páncreas Artificial". A partir de distintos enfoques de la teoría de control, teniendo en cuenta las restricciones y dificultades que presenta este sistema, se presentaron las siguientes contribuciones:

$\checkmark$ Una plataforma virtual para la simulación y ensayos pre-clínicos y la validación de estrategias de control glucémico. Se compone de escenarios realistas considerando comidas mixtas, variabilidad intra- e inter-paciente, modelos de las tecnologías actuales, entre otros aspectos. Se desarrolló de forma tal que su uso sea accesible a colegas y con características modulares que facilitan su ampliación. Este trabajo permitió diversas colaboraciones dentro y por fuera del grupo de trabajo. Se participó en el desarrollo de un modelo LPV de orden reducido orientado al control que contemple la variación intra-paciente. A su vez, se participó en el desarrollo de un simulador con aritmética intervalar que contempla diferentes modelos del sistema glucosa-insulina.

$\checkmark$ Una terapia de control posprandial a lazo abierto para compensar comidas de alto nivel glucémico, denominada Super-Bolo Automático basado en IOB.

$\checkmark$ Una terapia de modulación de insulina basal durante el ejercicio para proteger al sujeto tanto de posibles hipoglucemias debido al aumento de sensibilidad insulínica como de hiperglucemias a causa de la hipoinsulinemia.

$\checkmark$ Participación en el desarrollo de una técnica de transición entre lazo abierto y lazocerrado para sistemas híbridos de Páncreas Artificial durante ensayos clínicos.

$\checkmark$ Participación en el desarrollo y ensayo clínico de un algoritmo de control automático de glucosa sin bolos previos para las comidas.

$\checkmark$ Una estrategia para sistemas de control a lazo cerrado puro que protege al sujeto de hipoglucemias vía limitaciones en la tasa decreciente de glucosa.

En el trascurso de elaboración de esta tesis se tuvo un amplio conocimiento del estado del arte, de las tecnologías circundantes y de las técnicas desarrolladas para el tratamiento de la diabetes. 
Como trabajo a futuro, se desea realizar la adaptación de controladores para su utilización tanto en ensayos clínicos como en forma ambulatoria, buscando así reducir la brecha entre las más recientes contribuciones teóricas y su accesibilidad por parte de los destinatarios finales.

Partiendo de la experiencia obtenida durante el doctorado, se busca trabajar en dos grandes ejes: en primer lugar, en el desarrollo de estrategias de lazo cerrado puro para el control de glucosa mediante la combinación de una bomba de infusión continua de insulina con un monitor continuo de glucemia, y en segundo lugar en la migración de las estrategias a una plataforma para su ensayo en el ámbito clínico y/o ambulatorio. Se definen los siguientes objetivos específicos:

O1 Estudiar y desarrollar estrategias de control de lazo cerrado puro en base a un controlador con estructura MPC conmutado. Estudio de diferentes formas de implementar un controlador MPC a partir de la literatura [209]. Desarrollo de un controlador MPC conservador para la etapa nocturna/ayuno, y otro agresivo para la compensación de comidas. Estudiar la implementación de restricciones en la estructura interna de los controladores para asegurar un control glucémico más seguro, en base al algoritmo ARG 103.

O2 Estudiar y desarrollar estimadores de la excursión de glucosa posprandial y/o detectores de comida. A partir de trabajos del grupo [175, desarrollar e implementar un detector de comidas para realizar un anuncio automático de las mismas al controlador. Mediante el mismo, establecer las reglas de conmutación entre un controlador conservador y otro agresivo.

O5 Desarrollo de una plataforma de hardware y software de código abierto para pruebas clínicas del páncreas artificial. Puesta en marcha y comunicación de una plataforma basada en Andorid APS (AAPS) [210] mediante la conexión inalámbrica de monitores continuos de glucosa y bombas de insulina.

O6 Pruebas Clínicas de la Plataforma y Controlador. Pruebas clínicas hospitalarias en pacientes pediátricos (Hospital Garrahan) y semi-ambulatorias en pacientes adultos (Hospital Italiano) tanto de la conectividad y funcionamiento a lazo cerrado de la plataforma como de los nuevos algoritmos embebidos en la misma. 


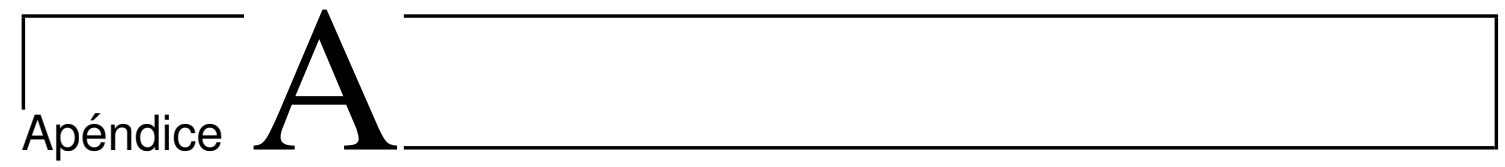

Lista de comidas mixtas 


\begin{tabular}{|c|c|c|}
\hline \#Comida & Composición & $\mathrm{CHO}(\mathrm{g})$ \\
\hline 01 & Leche, arroz blanco, pera, galletitas de salvado, queso light y aceite & 52 \\
\hline 02 & Leche, arroz blanco, pera, galletitas de salvado, aceite & 111 \\
\hline 03 & Leche, pan blanco, queso light, manteca y aceite & 52 \\
\hline 04 & Leche, pan blanco, queso light, manteca y aceite & 52 \\
\hline 05 & Pasta con poca cantidad de aceite de girasol & 75 \\
\hline 06 & Pasta con gran cantidad de aceite de girasol & 75 \\
\hline 07 & Arroz con leche, azúcar y canela & 50 \\
\hline 08 & Porotos, pan integral, salame y queso & 50 \\
\hline 09 & Pasta con Psyllium & 52 \\
\hline 10 & Barra de cebada & 25 \\
\hline 11 & Barra de avena & 25 \\
\hline 12 & Barra de chocolate con frambuesa & 10 \\
\hline 13 & Batido de soja y chocolate & 10 \\
\hline 14 & Barra de pasta de maní y chocolate & 25 \\
\hline 15 & Batido de soja, fructuosa y chocolate & 25 \\
\hline 16 & Chips proteínicos de soja salados & 25 \\
\hline 17 & Spaghetti de soja & 25 \\
\hline 18 & Cebada hervida (bajo IG) & 50 \\
\hline 19 & Puré de papas (alto IG) & 50 \\
\hline 20 & Papa al horno, pechuga de pavo y gelatina & 45 \\
\hline 21 & Arroz hervido, choclo y pechuga de pavo & 50 \\
\hline 22 & Pan blanco, huevos, margarina y jugo de naranja & 50 \\
\hline 23 & Suplemento nutricional en polvo & 50 \\
\hline 24 & Pasta con salsa de tomate & 50 \\
\hline 25 & Pasta con salsa de tomate y aceite & 50 \\
\hline 26 & Pasta con salsa de tomate, aceite y Psyllium & 50 \\
\hline 27 & Comida con alto contenido de $\mathrm{CHO}$ & 93 \\
\hline 28 & Comida con alto contenido de gasa & 27 \\
\hline 29 & Pasta con aceite de girasol & 75 \\
\hline 30 & Desayuno 1 & 120 \\
\hline 31 & Desayuno 2 & 70 \\
\hline 33 & Desayuno 3 & 50 \\
\hline 34 & Omelette de queso, pan y margarina & 38 \\
\hline 35 & Spaguetti con tomate, queso y lentejas & 87 \\
\hline 36 & Copitos de maíz con leche, pan y margarina & 104 \\
\hline 37 & Avena con leche, pan y margarina & 62 \\
\hline 38 & Cebada con leche, mermelada de frutilla y jugo de naranja & 69 \\
\hline 39 & Cereal de fruta y fibra con leche, ananá y melón & 50 \\
\hline 40 & Cereal con alto contenido de fibra con lecho, frutillas y pomelo & 42 \\
\hline 41 & Omelette de huevo, pan, espinaca, morrón y tomate grillado & 20 \\
\hline 42 & Omelette de huevo, miel, pan, espinaca y morrón & 15 \\
\hline 43 & Pan, leche, azúcar negra, banana y jugo de naranja & 47 \\
\hline 44 & Muffin con pasta de maní & 27 \\
\hline 45 & Golosina, leche, helado y crema doble & 80 \\
\hline 46 & Jarabe, leche descremada y helado sin grasa & 80 \\
\hline 47 & Galletitas con yogurt sin grasa & 94 \\
\hline 48 & Copos de trigo, queso fresco y leche & 80 \\
\hline 49 & Galletas de trigo, leche descremada, pan integral, frutas, pollo y vegetales 1 & 123 \\
\hline 50 & Galletas de trigo, leche descremada, pan integral, frutas, pollo y vegetales 2 & 126 \\
\hline 51 & Lasaña de vegetales, postre de crema y jugo de naranja & 27 \\
\hline 52 & Lasaña de vegetales, postre de leche descremada y jugo de naranja & 120 \\
\hline 53 & Arroz blanco & 75 \\
\hline 54 & Copos de cebada $100 \%$ & 75 \\
\hline 55 & Copos de cebada $50 \%$ & 75 \\
\hline 56 & Copos de cebada $30 \%$ & 75 \\
\hline 57 & Pan blanco & 50 \\
\hline 58 & Pan integral & 50 \\
\hline 59 & Pan de centeno $\beta$-glucan & 50 \\
\hline 60 & Pasta integral & 50 \\
\hline
\end{tabular}

Tabla A.1: Biblioteca de comidas mixtas. 


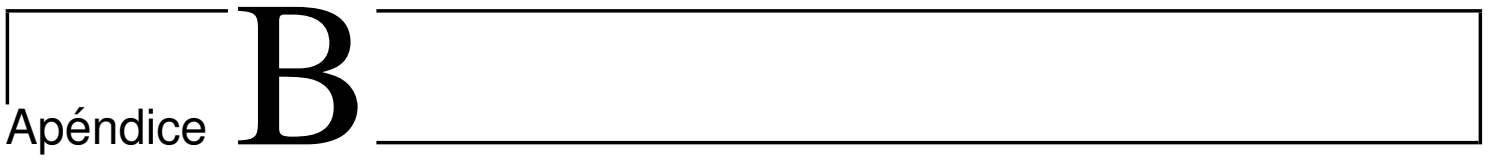

\section{Plataforma Android para ensayos clínicos}

En la actualidad existen diversas implementaciones de Páncreas Artificial "DIY" (Do It Yourself: hazlo tu mismo). Esto quiere decir que el paciente no adquiere un producto finalizado, sino que arma su propio sistema con las distintas alternativas de dispositivos y controladores disponibles y lo pone en funcionamiento por su cuenta. Las implementaciones que se destacan hoy en día son OpenAPS, Loop y Android APS. En particular OpenAPS se ejecuta sobre una "pequeña computadora", por ejemplo una Intel Edison, una Raspberry Pi0W o Pi3. El algoritmo que utiliza se llama "oref0" en su primera versión, que antecede a "oref1", la última versión estable. Tiene funciones como habilitar la detección de cambios en la sensibilidad a la insulina, asistencia para las comidas y cálculo dinámico de absorción de carbohidratos, así como también comidas sin anuncio y alertas cuando se requiere acción adicional por parte del usuario. De OpenAPS se desprende su versión modular para Android, denominada AndroidAPS (AAPS) [123], que conserva el mismo algoritmo.

A continuación se presenta un trabajo realizado dentro del grupo de trabajo a partir de la dirección de una estudiante de grado. El objetivo del presente apéndice es introducir una alternativa de PA de código abierto, como es AAPS, y realizar su puesta en funcionamiento y verificación. Como grupo de investigación se plantearon las pruebas del sistema AAPS como punto de partida para luego implementar y probar el algoritmo ARG desarrollado en el país 20, 159].

\section{B.1. Plataforma InsuMate}

Para la implementación de AAPS se requiere como mínimo un CGM, un celular, una bomba y el entorno de desarrollo Android Studio (por lo tanto una computadora) de modo de poder descargar la aplicación AAPS al celular.

En particular, para el presente trabajo y a lo que hardware respecta, se utilizó un celular Motorola Moto G5 XT1671 para la implementación del software y algoritmo de control. Se dispone del sistema CGM Dexcom G4, compuesto por sensor, transmisor y receptor y de una bomba modelo Accu-Chek Spirit Combo con su respectivo lector de infrarrojo Smart Pix Model 02, el cual permite realizar configuraciones de este modelo específico de bomba. Los componentes utilizados pueden observarse en la Fig. B.1. En cuanto a lo que respecta al software, se eligió como Sistema Operativo (SO) principal Ubuntu. Se tuvo que hacer la excepción para el uso del programa de configuración de la bomba, el cual requiere Windows para su funcionamiento. En Ubuntu (versión 16.04 LTS - kernel 4.15.0-33-generic), se utilizó Git (versión 2.7.4) para descargar el repositorio y guardar versiones del código en caso de modificarlo; Android Studio (versión 3.1.2) para desarrollar, ejecutar y depurar el código; MongoDB shell (versión v4.0.1) para obtener la información del sistema almacenada en la base de datos (DB). Por otro lado, en Windows 7 


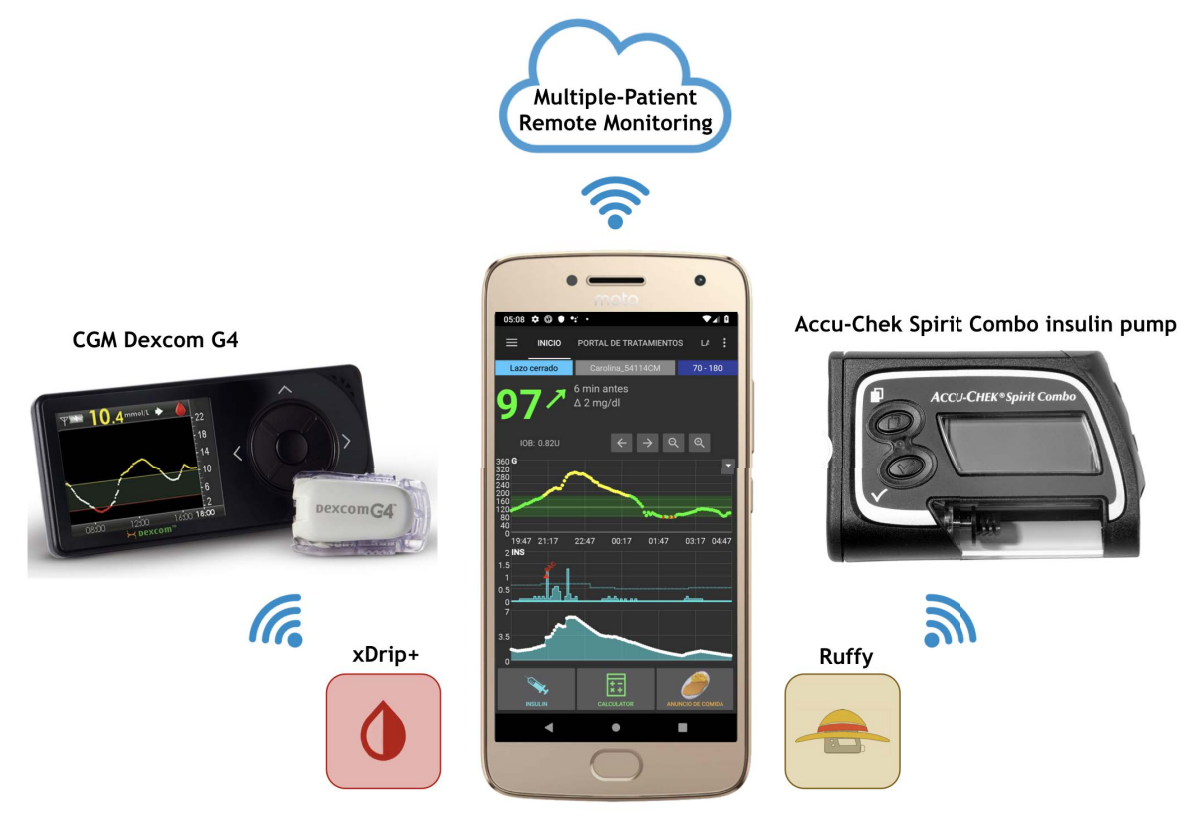

Figura B.1: Sistema AAPS: Bomba, Android APS, receptor del sensor y NightScout

(versión Home Basic - Service Pack 1) se instaló el programa de configuración de la bomba denominado ACCU-CHEK 360 Insulin Pump Configuration Software (versión 1.0.4.2907).

\section{B.2. Puesta en funcionamiento del sistema AAPS}

Para poner en funcionamiento el sistema se requieren múltiples pasos. Se deben configurar las aplicaciones en el celular, el sitio web de monitoreo (NightScout) y las conexiones tanto de la bomba como del sensor. Primero se "rooteó" el celular, es decir se obtuvo control privilegiado sobre el mismo para poder instalar la versión de Android Lineage OS 14.1, ya que AAPS no funciona correctamente con la versión 8.1 de Android pre-instalada en el celular provisto. Luego se instalaron en la computadora el sistema de control de versiones Git y el entorno de desarrollo Android Studio. Con las herramientas ya disponibles se prosiguió a descargar los repositorios de las aplicaciones, instalarlas y configurarlas.

El sistema se puede dividir según los dispositivos físicos (celular, bomba, CGM) que lo componen, como puede observarse en la Fig. B.2 representados mediante rectángulos. Por otra parte se encuentra el software, representado por óvalos, correspondientes a las aplicaciones y el sitio web de monitoreo. La aplicación principal es AAPS, la cual tiene múltiples configuraciones, como tipos de perfil de usuario, tipos de curva de insulina, tipos de bomba, opción de detección de sensibilidad, elección de lazo abierto o cerrado, objetivos y tratamientos, entre otras. A su vez se configuran las conexiones con las aplicaciones $\mathrm{xDrip}+\mathrm{y}$ NightScout. $\mathrm{xDrip}+$ es la aplicación encargada de recibir los datos del CGM. Se debe configurar para que tome los datos del sensor correspondiente y para que suba los datos de glucosa al sitio web de monitoreo. A su vez, se comunica con la aplicación central para proporcionar las medidas de glucosa recibidas por el receptor. Esta aplicación se comunica vía Bluetooth al sistema CGM, como se observa en la Fig. B.2. NightScout es el sitio web de monitoreo. La aplicación central le comunica los datos mediante conexión a Internet. En el sitio se pueden observar los datos del sistema, como lo son la glucosa, insulina, estado de la bomba, estado del sistema y el porqué de la toma de decisiones, entre otros. Para poner en funcionamiento el mismo se debe crear el sitio, por ejemplo en la plataforma Heroku, y realizar las configuraciones pertinentes. En lo que a la bomba 


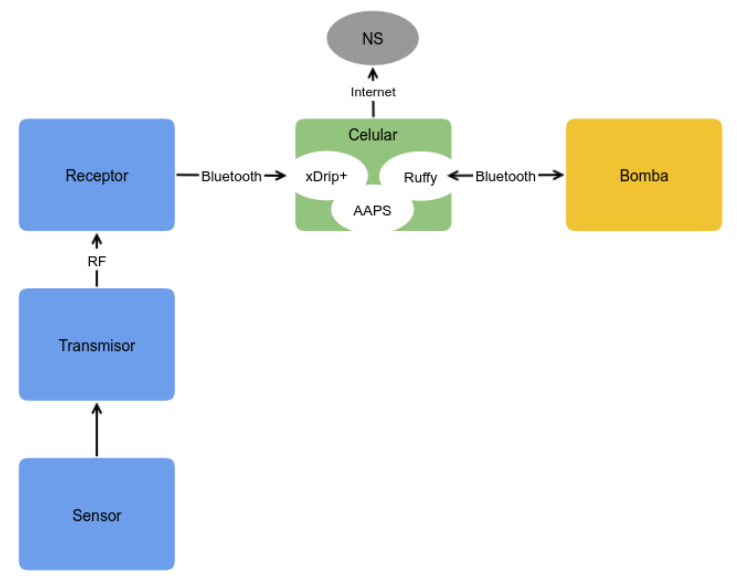

Figura B.2: Dispositivos y aplicaciones del sistema Android APS.

respecta, se configura con un software específico y un lector de infrarrojo denominado Smart Pix. Independientemente de la configuración generada, el celular y la bomba se comunican por Bluetooth mediante la aplicación Ruffy (Fig. B.2). La misma realiza la conexión a la bomba sólo una vez y esta se establece permanentemente, exceptuando el caso que se desvinculen manualmente los dispositivos. Durante la etapa de configuración y luego teniendo el sistema en funcionamiento, se analizaron las partes que lo componen y sus datos.

Se debieron realizar modificaciones y programas adicionales para facilitar las pruebas iniciales. En primer lugar, el sistema no funciona sin tener medidas de glucosa. Ante esto se podría probar sólo con CGM reales, lo cual carece de sentido práctico. Fue necesario desarrollar una solución para la generación de un CGM virtual. Mediante la ejecución de un programa en lenguaje Python se sube cada cierto tiempo un valor de glucosa a partir de un vector. Al poder utilizarse vectores obtenidos de simulaciones o de un sensor real, es posible analizar al sistema en su completo funcionamiento y realizar comparaciones con otras plataformas.

Para ensayar la plataforma se tuvo que modificar aspectos de la programación del AAPS. Dado que Android APS tiene una configuración de objetivos que bloquean distintos aspectos del sistema para acompañar al usuario en el proceso de aprendizaje del sistema, poder utilizar todas las funcionalidades lleva semanas. Por esta razón es que se modificó el código de modo de evitar dichos objetivos y tener acceso al sistema completo. Al algoritmo de control realizado por la comunidad de OpenAPS se lo denomina "oref0" en su primer versión y en principio se verificó la posibilidad de aplicarle modificaciones. Se realizó un mínimo cambio en el código, con el objetivo de corroborar si es posible alterar la cantidad de insulina que se inyecta, lo cual se pudo lograr sin inconvenientes. Por otra parte, es fundamental en cualquier sistema que genera datos poder obtener los mismos de forma ordenada. Para ello se realizaron consultas a la DB ya integrada en el sistema y se utilizó la herramienta online "mLab" disponible en mongoDB. Finalmente se realizó un manual de Android APS enfocado al desarrollador y otro enfocado al usuario.

\section{B.3. Resultados}

Tras la puesta en marcha y hacer los ajustes correspondientes, se apuntó a una prueba piloto del sistema, tanto a lazo abierto como a lazo cerrado. En el mes de julio de 2018 se realizaron pruebas del sistema completo. El sistema se encontraba funcionamiento junto con las aplicaciones AAPS, xDrip+ y Ruffy, y el sitio web de monitoreo NightScout. Se 


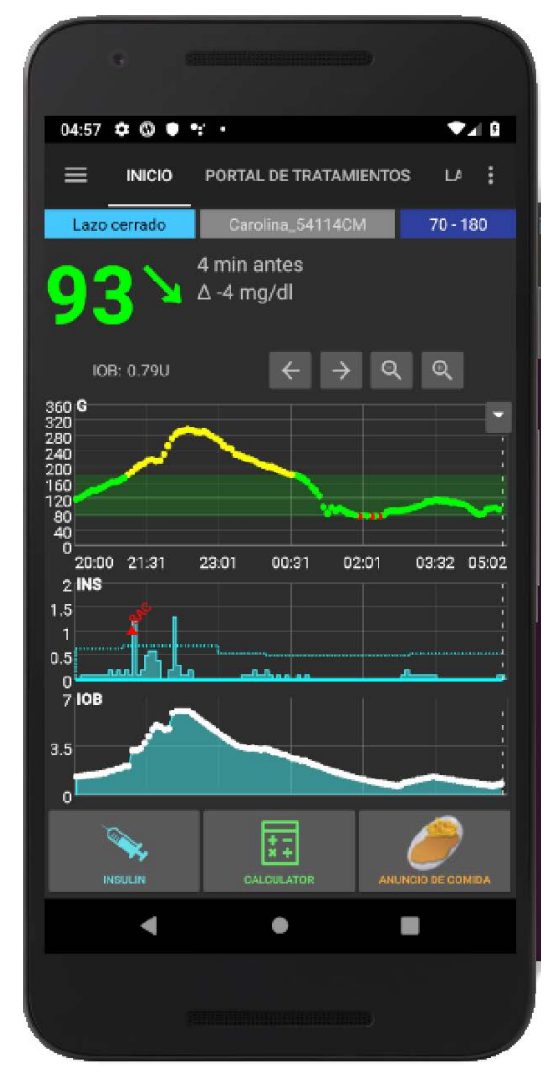

Figura B.3: Plataforma InsuMate durante su ensayo corriendo el algoritmo ARG.

colocó un CGM Dexcom G4 sobre una persona sin diabetes, por lo que la bomba se conectó al sistema y no al sujeto. El objetivo de las pruebas fue evaluar el sistema con un monitor continuo de glucosa real y no uno virtual. En la Fig. B.3 puede observarse la pantalla principal de la plataforma AAPS y 18 hs de funcionamiento. Puede observarse principalmente el nivel y la excursión de glucosa, junto con el suministro de insulina. Se analizaron y comprobaron las distancias entre receptor y celular, y entre celular y bomba. Se evaluaron escenarios con eventualidades, como la desconexión del sensor, que la bomba se quede sin batería o que el usuario erróneamente la apague. Se realizaron calibraciones del sensor, el cual las solicita cada doce horas y deben ser ingresadas desde el receptor. Por último, se analizó el funcionamiento y desempeño general de AAPS (ver Fig. B.4). La prueba duró 240 hs ininterrumpidas, verificándose el correcto funcionamiento del sistema durante el $93 \%$ del tiempo, lo cual se encuentra en el orden de lo obtenido con la plataforma DiAs (provista por la Universidad de Virginia) en las pruebas realizadas por el equipo de trabajo en noviembre de 2017 en el Hospital Italiano de Buenos Aires.

Se apuntó a la implementación de una plataforma abierta y transparente del tipo DIY debido a que posee las características necesarias para futuros ensayos clínicos de estrategias de control glucémico. El AAPS tiene una gran diversidad de usuarios en el mundo y una comunidad de soporte online activa. Además presenta la gran ventaja de poseer conexión con el sensor Dexcom G4 y la bomba Accu-Chek, que corresponden al equipamiento que se disponía. La implementación del sistema y las pruebas preliminares permitieron tener una comprensión más profunda de la plataforma. Se deben tener muchas consideraciones a la hora de realizar los ensayos clínicos, ya sea desde la estabilidad del sistema, tener en cuenta posibles eventualidades como lo son la desconexión de los dispositivos, posibles fallas del sistema, entre otras. Su implementación modular permite que se agreguen las prestaciones pertinentes (nuevos algoritmos de control, capas de seguridad, entre otros) a la hora de disponer de la plataforma. 


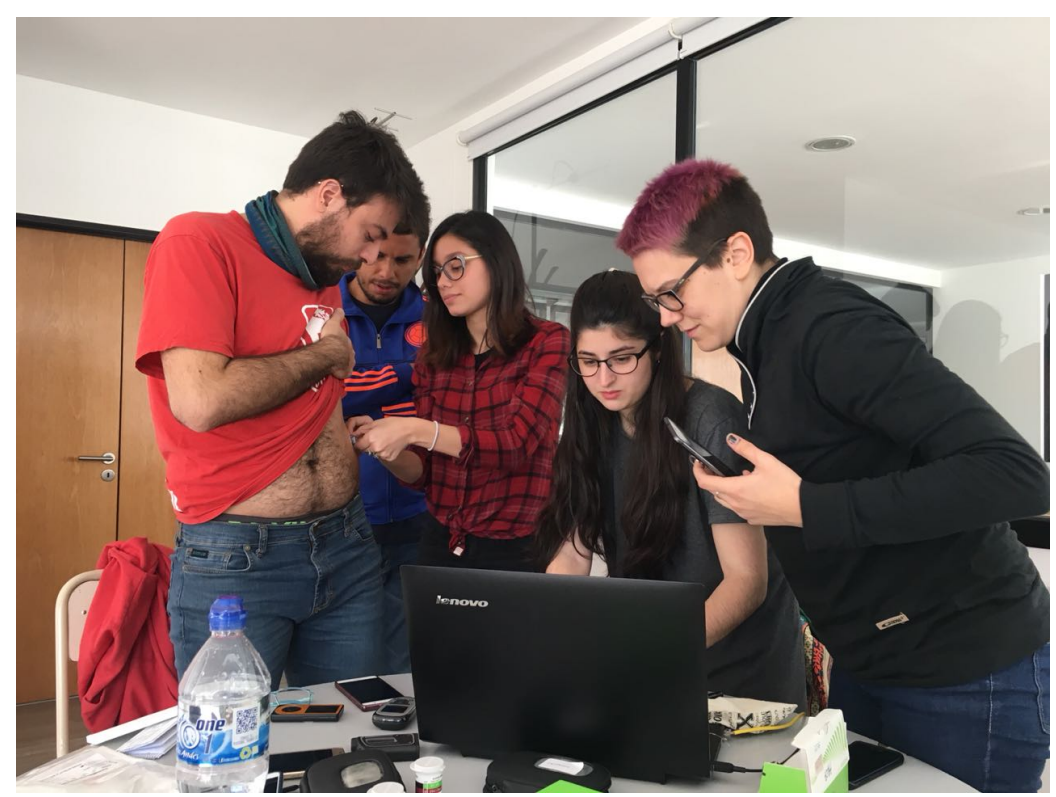

Figura B.4: Equipo de trabajo colocando y configurando el sensor Dexcom G4 para el ensayo de la plataforma.

Se realizaron ensayos de la plataforma InsuMate con el algoritmo ARG (Fig. B.5). El sistema base (InsuMate, Dexcom G4 y Roche Spirit Combo) fue probado durante 240hs, mostrando un adecuado funcionamiento del lazo-cerrado durante el $93 \%$ del tiempo total. En cuanto al algoritmo, se probó una versión del ARG con técnicas de mitigación de desconexión utilizando las mediciones de glucosa de los ensayos clínicos. El error relativo promedio en el total de insulina administrada con respecto a la implementación del controlador en Matlab fue de 5,37\%, mientras que la diferencia con los resultados de los ensayos clínicos con la implementación basada en DiAs fue de 6,94\%. Se probó una versión del sistema de tratamiento MDI de forma ambulatoria mediante un sensor FreeStyle Libre y con adaptador MiaoMiao durante 13 días. Se introducía manualmente las inyecciones de bolos y se monitoreaba la evolución de la estimación de IOB. Los resultados obtenidos indicaron un funcionamiento satisfactorio como sistema de monitoreo en el 91,90\% del tiempo total.

\begin{tabular}{|c|c|c|c|c|}
\hline $\begin{array}{l}\text { Prueba de } \\
\text { conexión del } \\
\text { Sistema base } \\
\text { (julio 2018) }\end{array}$ & $\begin{array}{l}\text { Diferencia de un } \\
5,37 \%\end{array}$ & $\begin{array}{l}\text { Comparación del } \\
\text { algoritmo ARG en } \\
\text { InsuMAte vs } \\
\text { ensayos clínicos } \\
\text { (oct 2019) }\end{array}$ & $\begin{array}{l}13 \text { días, funcionó } \\
\text { correctamente un } \\
91,90 \%\end{array}$ & $\begin{array}{l}\text { Ensayo clínico } \\
\text { ambulatorio del } \\
\text { algoritmo ARG } \\
\text { en InsuMate } \\
\text { (abril 2020) }\end{array}$ \\
\hline $\begin{array}{l}\text { Lazo-cerrado opera } \\
\text { adecaudamente } 93 \% \\
\text { dentro de } 240 \mathrm{hs}\end{array}$ & $\begin{array}{l}\text { Comparación del } \\
\text { algoritmo ARG en } \\
\text { InsuMAte vs Matlab } \\
\text { (sep 2019) }\end{array}$ & $\begin{array}{l}\text { Diferencia de } \\
\text { un } 6,94 \%\end{array}$ & $\begin{array}{l}\text { Prueba ambulatoria } \\
\text { preliminar de MDI } \\
\text { (nov 2019) }\end{array}$ & \\
\hline
\end{tabular}

Figura B.5: Línea tempral de los resultados de la plataforma InsuMate ante diferentes configuraciones

\section{B.4. Conclusiones}

El presente apéndice apunta a describir el proceso de puesta en funcionamiento de una plataforma de código abierto que brinda a los pacientes con DMT1 la disponibilidad de un sistema a lazo cerrado gratuito y adaptable. La misma está implementada en Android y permite comunicarse y comandar una bomba de infusión subcutánea de insulina y un 
monitor continuo de glucosa. Se logró hacer una prueba piloto de $240 \mathrm{hs}$ con resultados satisfactorios. Se espera realizar pruebas con dispositivos comercializados nacionalmente como por ejemplo el CGM Freestyle Libre. Por otra parte, se realizará la programación del algoritmo ARG dentro de AAPS, con el objetivo de realizar pruebas clínicas en pacientes pediátricos en nuestro país, cuyo protocolo ya fue aprobado por el Comité de Ética del Hospital Garrahan. 


\section{Bibliografía}

[1] J Bondia, J Vehí, C C Palerm, and P Herrero. El Páncreas Artificial: Control Automático de Infusión de Insulina en Diabetes Mellitus Tipo 1. Revista Iberoamericana de Automática e Informática Industrial, 7(2):5-20, 2010.

[2] ADA. ADA Standards of Medical Care in Diabetes. Diabetes Care, 42(Supplement 1), 2019.

[3] Walter A. Müller, Gerald R. Faloona, Eugenio Aguilar-Parada, and Roger H. Unger. Abnormal alpha-cell function in diabetes. New England Journal of Medicine, 283(3):109-115, 1970.

[4] International Diabetes Federation. IDF diabetes atlas, 9th edn. Brussels, Belgium, 2019 .

[5] Pamela Martyn-Nemeth, Sarah Schwarz Farabi, Dan Mihailescu, Jeffrey Nemeth, and Laurie Quinn. Fear of hypoglycemia in adults with type 1 diabetes: impact of therapeutic advances and strategies for prevention - a review. Journal of Diabetes and its Complications, 30(1):167-177, 2016.

[6] Ministerio de Ciencia, tecnología e Innovación Productiva. Argentina innovadora $2020,2015$.

[7] Ministerio de Salud y Desarrollo Social. 4ta encuesta nacional de factores de riesgo, 2018 .

[8] Peter Adams. The impact of brief high-intensity exercise on blood glucose levels. Diabetes, Metabolic Syndrome and Obesity: Targets and Therapy, 6:113, 2013.

[9] M. J. Fowler. Microvascular and macrovascular complications of diabetes. Clinical Diabetes, 26(2):77-82, 2008.

[10] Ritu Lakhtakia. The history of diabetes mellitus. Sultan Qaboos University Medical Journal, 13(3):368-370, 2013.

[11] Celeste C. Quianzon and Issam Cheikh. History of insulin. Journal of Community Hospital Internal Medicine Perspectives, 2(2):18701, 2012.

[12] John Pickup and Harry Keen. Continuous subcutaneous insulin infusion at 25 years: evidence base for the expanding use of insulin pump therapy in type 1 diabetes. Diabetes care, 25:593-598, 2002.

[13] Jennifer Sherr and William V Tamborlane. Past, present, and future of insulin pump therapy: better shot at diabetes control. The Mount Sinai journal of medicine, New York, 75:352-361, 2008. 
[14] J Walsh and R Roberts. Pumping insulin: everything you need for success on a smart insulin pump. Torrey Pines Pr, 2006.

[15] Jill Weissberg-Benchell, Jeanne Antisdel-Lomaglio, and Roopa Seshadri. Insulin pump therapy: a meta-analysis. Diabetes care, 26:1079-1087, 2003.

[16] Signe Schmidt and Kirsten Nørgaard. Bolus Calculators. Journal of Diabetes Science and Technology, 8(5):1035-1041, 2014.

[17] Eda Cengiz, Jennifer L Sherr, Stuart A Weinzimer, and William V Tamborlane. Newgeneration diabetes management: glucose sensor-augmented insulin pump therapy. Expert review of medical devices, 8:449-458, 2011.

[18] J Walsh, R Roberts, and T Bailey. Guidelines for optimal bolus calculator settings in adults. Journal of Diabetes Science and Technology, 5(1):129-135, 2011.

[19] Gary Scheiner, Robert J Sobel, Daphne E Smith, Anthony J Pick, Davida Kruger, Jacqueline King, and Karen Green. Insulin Pump Therapy: Guidelines for Successful Outcomes. The Diabetes Educator, 35(2 suppl):29S-41S, 2009.

[20] Ricardo Sánchez-Peña, Demián García-Violini, Marcela Moscoso-Vásquez, Patricio Colmegna, Fabricio Garelli, Hernán De Battista, Emilia Fushimi, Marc Breton, Nicolás Rosales, Enrique Campos-Náñez, Valeria Beruto, Paula Scibona, Cintia Rodriguez, Javier Giunta, Ventura Simonovich, Waldo H. Belloso, Daniel Cherñavvsky, and Luis Grosembacher. Artificial Pancreas: Clinical Study in Latin America Without Premeal Insulin Boluses. Journal of Diabetes Science and Technology, 12(5):914-925, 2018.

[21] Natalie Allen and Anshu Gupta. Current diabetes technology: Striving for the artificial pancreas. Diagnostics, 9(1):31, 2019.

[22] Kamuran Turksoy, Nicole Frantz, Laurie Quinn, Magdalena Dumin, Jennifer Kilkus, Brooks Hibner, Ali Cinar, and Elizabeth Littlejohn. Automated insulin delivery - the light at the end of the tunnel. The Journal of Pediatrics, 186:17-28.e9, 2017.

[23] Paul Downie. Practical aspects of capillary blood glucose monitoring: a simple guide for primary care. Diabetes \& Primary Care, 15(3):149-153, 2013.

[24] David C Klonoff. Continuous glucose monitoring: roadmap for 21st century diabetes therapy. Diabetes care, 28:1231-1239, 2005.

[25] Sandeep Kumar Vashist. Continuous glucose monitoring systems: A review. Diagnostics (Basel, Switzerland), 3:385-412, 2013.

[26] David Olczuk and Ronny Priefer. A history of continuous glucose monitors (cgms) in self-monitoring of diabetes mellitus. Diabetes $\&$ metabolic syndrome, 12:181-187, 2018.

[27] Eun-Hyung Yoo and Soo-Youn Lee. Glucose biosensors: an overview of use in clinical practice. Sensors (Basel, Switzerland), 10:4558-4576, 2010.

[28] Ananda Basu, Simmi Dube, Michael Slama, Isabel Errazuriz, Jose Carlos Amezcua, Yogish C Kudva, Thomas Peyser, Rickey E Carter, Claudio Cobelli, and Rita Basu. Time lag of glucose from intravascular to interstitial compartment in humans. Diabetes, 62:4083-4087, 2013. 
[29] Florian Reiterer, Philipp Polterauer, Michael Schoemaker, Guenther SchmelzeisenRedecker, Guido Freckmann, Lutz Heinemann, and Luigi Del Re. Significance and reliability of mard for the accuracy of cgm systems. Journal of diabetes science and technology, 11:59-67, 2017.

[30] Boris P Kovatchev, Stephen D Patek, Edward Andrew Ortiz, and Marc D Breton. Assessing sensor accuracy for non-adjunct use of continuous glucose monitoring. Diabetes technology $\& 5$ therapeutics, 17:177-186, 2015.

[31] M J Lenhard and G D Reeves. Continuous subcutaneous insulin infusion: a comprehensive review of insulin pump therapy. Archives of internal medicine, 161:2293$2300,2001$.

[32] Luis G Jahn, Jorge J Capurro, and Brian L Levy. Comparative dose accuracy of durable and patch insulin infusion pumps. Journal of diabetes science and technology, 7:1011-1020, 2013.

[33] Stefan Pleus, Ulrike Kamecke, Delia Waldenmaier, and Guido Freckmann. Reporting insulin pump accuracy: Trumpet curves according to iec 60601-2-24 and beyond. Journal of diabetes science and technology, 13:592-596, 2019.

[34] P Schaepelynck, P Darmon, L Molines, M F Jannot-Lamotte, C Treglia, and D Raccah. Advances in pump technology: insulin patch pumps, combined pumps and glucose sensors, and implanted pumps. Diabetes $\&$ metabolism, 37 Suppl 4:S85-S93, 2011.

[35] Thomas Danne, Olga Kordonouri, Martin Holder, Holger Haberland, Sven Golembowski, Kerstin Remus, Sara Bläsig, Tanja Wadien, Susanne Zierow, Reinhard Hartmann, and Andreas Thomas. Prevention of Hypoglycemia by Using Low Glucose Suspend Function in Sensor-Augmented Pump Therapy. Diabetes Technology and Therapeutics, 13(11):1129-1134, 2011.

[36] Mary B. Abraham, Jennifer A. Nicholas, Grant J. Smith, Janice M. Fairchild, Bruce R. King, Geoffrey R. Ambler, Fergus J. Cameron, Elizabeth A. Davis, and Timothy W. Jones. Reduction in hypoglycemia with the predictive low-Glucose management system: A long-term randomized controlled trial in adolescents with type 1 diabetes. Diabetes Care, 41(2):303-310, 2018.

[37] Banshi D Saboo and Praful A Talaviya. Continuous subcutaneous insulin infusion: practical issues. Indian journal of endocrinology and metabolism, 16:S259-S262, 2012.

[38] Deborah Young-Hyman, Mary de Groot, Felicia Hill-Briggs, Jeffrey S Gonzalez, Korey Hood, and Mark Peyrot. Psychosocial care for people with diabetes: A position statement of the american diabetes association. Diabetes care, 39:2126-2140, 2016.

[39] Victoria Franklin. Influences on technology use and efficacy in type 1 diabetes. Journal of diabetes science and technology, 10:647-655, 2016.

[40] C Farrington. Psychosocial impacts of hybrid closed-loop systems in the management of diabetes: a review. Diabetic medicine : a journal of the British Diabetic Association, 35:436-449, 2018.

[41] Susana R Patton and Mark A Clements. Psychological reactions associated with continuous glucose monitoring in youth. Journal of diabetes science and technology, 10:656-661, 2016. 
[42] Lutz Heinemann and Edward Krisiunas. Diabetes technology and waste: A complex problem piling up! Journal of Diabetes Science and Technology, 13(5):815-816, 2019.

[43] E B Marliss, F T Murray, E F Stokes, B Zinman, A F Nakhooda, A Denoga, B S Leibel, and A M Albisser. Normalization of glycemia in diabetics during meals with insulin and glucagon delivery by the artificial pancreas. Diabetes, 26:663-672, 1977.

[44] JDRF/NIDDK. Juvenile Diabetes Research Foundation Artificial Pancreas Consortium, 2013.

[45] Rozana Ramli, Monika Reddy, and Nick Oliver. Artificial Pancreas: Current Progress and Future Outlook in the Treatment of Type 1 Diabetes. Drugs, 79(10):1089-1101, 2019 .

[46] Francis J. Doyle, Lauren M. Huyett, Joon Bok Lee, Howard C. Zisser, and Eyal Dassau. Closed-loop artificial pancreas systems: Engineering the algorithms. Diabetes Care, 37(5):1191-1197, 2014.

[47] G M Steil, A E Panteleon, and K Rebrin. Closed-loop insulin delivery-the path to physiological glucose control. Advanced Drug Delivery Reviews, 56(2):125-144, 2004.

[48] B Wayne Bequette. Algorithms for a closed-loop artificial pancreas: the case for model predictive control. Journal of diabetes science and technology, 7:1632-1643, 2013.

[49] Jordan E. Pinsker, Joon Bok Lee, Eyal Dassau, Dale E. Seborg, Paige K. Bradley, Ravi Gondhalekar, Wendy C. Bevier, Lauren Huyett, Howard C. Zisser, and Francis J. Doyle. Randomized crossover comparison of personalized MPC and PID control algorithms for the artificial pancreas. Diabetes Care, 39(7):1135-1142, 2016.

[50] Lia Bally, Hood Thabit, and Roman Hovorka. Glucose-responsive insulin delivery for type 1 diabetes: The artificial pancreas story. International journal of pharmaceutics, 544:309-318, 2018.

[51] Martina Vettoretti and Andrea Facchinetti. Combining continuous glucose monitoring and insulin pumps to automatically tune the basal insulin infusion in diabetes therapy: a review. BioMedical Engineering OnLine, 18(1), 2019.

[52] Benyamin Grosman, Jacob Ilany, Anirban Roy, Natalie Kurtz, Di Wu, Neha Parikh, Gayane Voskanyan, Noa Konvalina, Chrystaleni Mylonas, Rebecca Gottlieb, Francine Kaufman, and Ohad Cohen. Hybrid closed-loop insulin delivery in type 1 diabetes during supervised outpatient conditions. Journal of diabetes science and technology, 10:708-713, 2016.

[53] Jenine Y Stone, Nalani Haviland, and Timothy S Bailey. Review of a commercially available hybrid closed-loop insulin-delivery system in the treatment of type 1 diabetes. Therapeutic delivery, 9:77-87, 2018.

[54] Ananda Basu, Simmi Dube, Sona Veettil, Michael Slama, Yogish C Kudva, Thomas Peyser, Rickey E Carter, Claudio Cobelli, and Rita Basu. Time lag of glucose from intravascular to interstitial compartment in type 1 diabetes. Journal of diabetes science and technology, 9:63-68, 2015.

[55] Ahmad Haidar. The Artificial Pancreas: How Closed-Loop Control Is Revolutionizing Diabetes. IEEE Control Systems, 36(5):28-47, 2016. 
[56] P. Colmegna and R. S. Sánchez Peña. Analysis of three T1DM simulation models for evaluating robust closed-loop controllers. Computer Methods and Programs in Biomedicine, 113(1):371-382, 2014.

[57] K D Barnard, T Wysocki, H Thabit, M L Evans, S Amiel, S Heller, A Young, R Hovorka, and Angela Consortium. Psychosocial aspects of closed- and open-loop insulin delivery: closing the loop in adults with type 1 diabetes in the home setting. Diabetic medicine : a journal of the British Diabetic Association, 32:601-608, 2015.

[58] Sverre Christian Christiansen, Anders Lyngvi Fougner, Oyvind Stavdahl, Konstanze Kölle, Reinold Ellingsen, and Sven Magnus Carlsen. A review of the current challenges associated with the development of an artificial pancreas by a double subcutaneous approach. Diabetes therapy : research, treatment and education of diabetes and related disorders, 8:489-506, 2017.

[59] Claudio Cobelli Ewart R. Carson. Modelling Methodology for Physiology and Medicine. Elsevier Science Publishing Co Inc, 2013.

[60] L J Chassin, M E Wilinska, and R Hovorka. Evaluation of glucose controllers in virtual environment: Methodology and sample application. Artificial Intelligence in Medicine, 32(3 SPEC.ISS.):171-181, 2004.

[61] M E Wilinska and R Hovorka. Simulation models for in silico testing of closedloop glucose controllers in type 1 diabetes. Drug Discovery Today: Disease Models, 5(4):289-298, 2008.

[62] J T Sorensen. A Physiologic Model of Glucose Metabolism in Man and its Use to Design and Asses Improved Insulin Therapies for Diabetes. PhD thesis, Massachusetts Institute of Technology, Cambridge, MA, USA, 1985.

[63] M. O. Larsen and B. Rolin. Use of the gottingen minipig as a model of diabetes, with special focus on type 1 diabetes research. ILAR Journal, 45(3):303-313, 2004.

[64] R N Bergman, L S Phillips, and C Cobelli. Physiologic evaluation of factors controlling glucose tolerance in man: measurement of insulin sensitivity and beta-cell glucose sensitivity from the response to intravenous glucose. Journal of Clinical Investigation, 68(6):1456-1467, 1981.

[65] R. N. Bergman. Toward physiological understanding of glucose tolerance: Minimalmodel approach. Diabetes, 38(12):1512-1527, 1989.

[66] Anirban Roy and Robert S Parker. Dynamic modeling of exercise effects on plasma glucose and insulin levels. Journal of diabetes science and technology, 1(3):338-347, 2007.

[67] Klaske van Heusden, Eyal Dassau, Howard C Zisser, Dale E Seborg, and Francis J Doyle III. Control-Relevant Models for Glucose Control Using a priori Patient Characteristics. IEEE Trans. Biomed. Eng., 59(7):1839-1849, 2012.

[68] Nicolas Magdelaine, Lucy Chaillous, Isabelle Guilhem, Jean-Yves Poirier, Michel Krempf, Claude H Moog, and Eric Le Carpentier. A long-term model of the glucoseinsulin dynamics of type 1 diabetes. IEEE transactions on bio-medical engineering, 62:1546-1552, 2015.

[69] R.Hovorka A N D Chassin, U Haueter, M Massi-Benedetti, M O Federici, T R Pieber, H C Schaller, L Schaupp, T Vering, and M E Wilinska. Nonlinear Model Predictive 
Control of Glucose Concentration in Subjects with Type 1 Diabetes. Physiological Measurement, 25:905-920, 2004.

[70] Malgorzata E Wilinska, Ludovic J Chassin, Carlo L Acerini, Janet M Allen, David B Dunger, and Roman Hovorka. Simulation Environment to Evaluate ClosedLoop Insulin Delivery Systems in Type 1 Diabetes. Journal of Diabetes Science and Technology, 4(1):132-144, 2010.

[71] Roman Hovorka, Daniela Elleri, Hood Thabit, Janet M Allen, Lalantha Leelarathna, Ranna El-Khairi, Kavita Kumareswaran, Karen Caldwell, Peter Calhoun, Craig Kollman, Helen R Murphy, Carlo L Acerini, Malgorzata E Wilinska, Marianna Nodale, and David B Dunger. Overnight closed-loop insulin delivery in young people with type 1 diabetes: a free-living, randomized clinical trial. Diabetes care, 37(5):1204-11, 2014 .

[72] Patricio Colmegna. Simulation 85 Control in Type 1 Diabetes. PhD thesis, ITBA, 2014.

[73] Chiara Dalla Man, Robert A. Rizza, and Claudio Cobelli. Meal simulation model of the glucose-insulin system. IEEE Transactions on Biomedical Engineering, 2007.

[74] Chiara Dalla Man, Davide M Raimondo, Robert A Rizza, and Claudio Cobelli. GIM, simulation software of meal glucose-insulin model. Journal of diabetes science and technology, 1(3):323-330, 2007.

[75] Chiara Dalla Man, Francesco Micheletto, Dayu Lv, Marc Breton, Boris Kovatchev, and Claudio Cobelli. The UVA/PADOVA type 1 diabetes simulator: New features. Journal of Diabetes Science and Technology, 8(1):26-34, 2014.

[76] R Visentin, C Dalla Man, B P Kovatchev, and C Cobelli. The University of Virginia/Padova Type 1 Diabetes Simulator Matches the Glucose Traces of a Clinical Trial. Diabetes Technol. Ther., 16(7):428-434, 2014.

[77] R Visentin, C Dalla Man, Y C Kudva, A Basu, and C Cobelli. Circadian Variability of Insulin Sensitivity: Physiological Input for In Silico Artificial Pancreas. Diabetes Technology \& Therapeutics, 17(1):1-7, 2015.

[78] R Visentin, C D Man, and C Cobelli. One-Day Bayesian Cloning of Type 1 Diabetes Subjects: Toward a Single-Day UVA/Padova Type 1 Diabetes Simulator. IEEE Transactions on Biomedical Engineering, 63(11):2416-2424, 2016.

[79] Mirko Messori, Jort Kropff, Simone Del Favero, Jerome Place, Roberto Visentin, Roberta Calore, Chiara Toffanin, Federico Di Palma, Giordano Lanzola, Anne Farret, Federico Boscari, Silvia Galasso, Angelo Avogaro, Patrick Keith-Hynes, Boris P Kovatchev, Daniela Bruttomesso, Lalo Magni, J Hans DeVries, Eric Renard, Claudio Cobelli, and for the AP@home consortium. Individually Adaptive Artificial Pancreas in Subjects with Type 1 Diabetes: A One-Month Proof-of-Concept Trial in Free-Living Conditions. Diabetes Technol. Ther., 19(10):560-571, 2017.

[80] R Hovorka. Closed-loop insulin delivery: From bench to clinical practice. Nature Reviews Endocrinology, 7(7):385-395, 2011.

[81] Patricio Colmegna, Ricardo S. Sanchez Peña, Ravi Gondhalekar, Eyal Dassau, and Francis J. Doyle. Reducing Risks in Type 1 Diabetes Using $H_{\infty}$ control. IEEE Transactions on Biomedical Engineering, 61(12):2939-2947, 2014. 
[82] Gregory P Forlenza. Ongoing debate about models for artificial pancreas systems and in silico studies. Diabetes technology \& therapeutics, 20:174-176, 2018.

[83] Marco Viceconti, Claudio Cobelli, Tarek Haddad, Adam Himes, Boris Kovatchev, and Mark Palmer. In silico assessment of biomedical products: The conundrum of rare but not so rare events in two case studies. Proceedings of the Institution of Mechanical Engineers. Part H, Journal of engineering in medicine, 231:455-466, 2017.

[84] P G Fabietti, V Canonico, M O Federici, M M Benedetti, and E Sarti. Control oriented model of insulin and glucose dynamics in type 1 diabetics. Med Biol Eng Comput, 44(1-2):69-78, 2006.

[85] Marc Breton and Boris Kovatchev. Analysis, modeling, and simulation of the accuracy of Continuous glucose sensors. Journal of Diabetes Science and Technology, $2(5): 853-862,2008$.

[86] L Magni, D M Raimondo, C Dalla Man, M Breton, S Patek, G De Nicolao, C Cobelli, and B P Kovatchev. Evaluating the Efficacy of Closed-Loop Glucose Regulation via Control-Variability Grid Analysis. J. Diabetes Sci. Technol., 2(4):630-635, 2008.

[87] Diego de Pereda, Sergio Romero-Vivo, Beatriz Ricarte, and Jorge Bondia. On the prediction of glucose concentration under intra-patient variability in type 1 diabetes: A monotone systems approach. Computer Methods and Programs in Biomedicine, 108(3):993-1001, 2012.

[88] Marcela Moscoso-Vasquez, Patricio Colmegna, and Ricardo S. Sanchez-Pena. Intrapatient dynamic variations in type 1 diabetes: A review. In 2016 IEEE Conference on Control Applications (CCA). IEEE, 2016.

[89] F. Porcellati, P. Lucidi, G. B. Bolli, and C. G. Fanelli. Thirty years of research on the dawn phenomenon: Lessons to optimize blood glucose control in diabetes. Diabetes Care, 36(12):3860-3862, 2013.

[90] Michele Schiavon, Chiara Dalla Man, Yogish C Kudva, Ananda Basu, and Claudio Cobelli. Quantitative estimation of insulin sensitivity in type 1 diabetic subjects wearing a sensor-augmented insulin pump. Diabetes care, 37:1216-1223, 2014.

[91] Maira García Jaramillo. Prediction of postprandial blood glucose under intra-patient variability and uncertainty and its use in the design of insulin dosing strategies for type 1 diabetic patients. PhD thesis, Universitat de Girona, 2011.

[92] C Toffanin, H Zisser, F J Doyle III, and E Dassau. Dynamic Insulin on Board: Incorporation of Circadian Insulin Sensitivity Variation. Journal of Diabetes Science and Technology, 7(4):928-940, 2013.

[93] Pau Herrero, Peter Pesl, Jorge Bondia, Monika Reddy, Nick Oliver, Pantelis Georgiou, and Christofer Toumazou. Method for automatic adjustment of an insulin bolus calculator: In silico robustness evaluation under intra-day variability. Computer Methods and Programs in Biomedicine, 119(1):1-8, 2015.

[94] Fabian León-Vargas, Fabricio Garelli, Hernán de Battista, and Josep Vehí. Postprandial response improvement via safety layer in closed-loop blood glucose controllers. Biomed. Signal Process Control, 16:80-87, 2015. 
[95] Gary Scheiner and Bret A Boyer. Characteristics of basal insulin requirements by age and gender in Type-1 diabetes patients using insulin pump therapy. Diabetes Research and Clinical Practice, 69:14-21, 2005.

[96] F León-Vargas, F Garelli, J Picó, H De Battista, and J Vehí. Postprandial blood glucose control using a hybrid adaptive PD controller with insulin-on-board limitation. Biomed. Signal Process Control, 8(6):724-732, 2013.

[97] Ahmad Haidar, Daniela Elleri, Kavita Kumareswaran, Lalantha Leelarathna, JANET M. ALLEN, Karen Caldwell, Helen R. Murphy, Malgorzata E. Wilinska, CARLO L. ACERINI, MARK L. EVANS, David B. Dunger, Marianna Nodale, and Roman Hovorka. Pharmacokinetics of Insulin Aspart in Pump-Treated SubjectsWith Type 1 Diabetes: Reproducibility and EffectofAge,Weight, and Duration of Diabetes. Diabetes Care, 36:173-174, 2013.

[98] F León-Vargas. Design and implementation of a closed-loop blood glucose control system in patients with type 1 diabetes. PhD thesis, Universitat de Girona, 2013.

[99] Chiara Toffanin, Roberto Visentin, Mirko Messori, Federico Di Palma, Lalo Magni, and Claudio Cobelli. Toward a run-to-run adaptive artificial pancreas: In silico results. IEEE transactions on bio-medical engineering, 65:479-488, 2018.

[100] Pau Herrero, Jorge Bondia, Cesar C. Palerm, Josep Vehí, Pantelis Georgiou, Nick Oliver, and Christofer Toumazou. A simple robust method for estimating the glucose rate of appearance from mixed meals. Journal of Diabetes Science and Technology, 6(1):153-162, 2012.

[101] C Barajas-Solano, J Bondia, R Calm, P Herrero, and J Vehí. A review of absorption models for mixed meals. In 5th International Conference on Advanced Treatments ES Technologies for Diabetes, 2012.

[102] Federación argentina de diabetes - https://www.fad.org.ar/.

[103] P. Colmegna, R. S. Sánchez Peña, and R. Gondhalekar. Linear parameter-varying model to design control laws for an artificial pancreas. Biomedical Signal Processing and Control, 40:204-213, 2018.

[104] Claudio Cobelli, Chiara Dalla Man, Giovanni Sparacino, Lalo Magni, Giuseppe De Nicolao, and Boris P. Kovatchev. Diabetes: Models, signals, and control. IEEE Reviews in Biomedical Engineering, 2:54-96, 2009.

[105] J B Lee, E Dassau, D E Seborg, and F. J. Doyle III. Model-Based personalization scheme of an artificial pancreas for type 1 diabetes applications. In American Control Conference (ACC), pages 2911-2916, Washington, DC, USA, 2013.

[106] Marcela Moscoso-Vasquez, Patricio Colmegna, Nicolas Rosales, Fabricio Garelli, and Ricardo Sanchez-Pena. Control-oriented model with intra-patient variations for an artificial pancreas. IEEE journal of biomedical and health informatics, 2020.

[107] L. Hinshaw, C. Dalla Man, D. K. Nandy, A. Saad, A. E. Bharucha, J. A. Levine, R. A. Rizza, R. Basu, R. E. Carter, C. Cobelli, Y. C. Kudva, and A. Basu. Diurnal pattern of insulin action in type 1 diabetes: Implications for a closed-loop system. Diabetes, 62(7):2223-2229, 2013. 
[108] Maira García-Jaramillo, Fabian León-Vargas, Nicolás Rosales, Andrés Molano, and Fabricio Garelli. Interval simulator of the glucose-insulin system. In Communications in Computer and Information Science, pages 686-695. Springer International Publishing, 2019.

[109] R Calm, M García-Jaramillo, J Bondia, M A Sainz, and J Vehí. Comparison of interval and Monte Carlo simulation for the prediction of postprandial glucose under uncertainty in type 1 diabetes mellitus. Computer Methods and Programs in Biomedicine, 104(3):325-332, 2011.

[110] M García-Jaramillo, R Calm, J Bondia, and J Vehí. Prediction of postprandial blood glucose under uncertainty and intra-patient variability in type 1 diabetes: a comparative study of three interval models. Computer methods and programs in biomedicine, 108:224-233, 2012.

[111] M. García-Jaramillo, J. S. Delgado, and F. León-Vargas. Glu4pred: A computational tool for design and testing of insulin therapies for patients with type 1 diabetes based on interval simulation. In VII Latin American Congress on Biomedical Engineering CLAIB 2016, Bucaramanga, Santander, Colombia, October 26th -28th, 2016, pages 337-340. Springer Singapore, 2017.

[112] Miguel A. Sainz, Joaquim Armengol, Remei Calm, Pau Herrero, Lambert Jorba, and Josep Vehi. Modal Interval Analysis. Springer International Publishing, 2014.

[113] P. Colmegna, R. S. Sanchez-Pena, and R. Gondhalekar. Control-oriented Linear Parameter-Varying model for glucose control in Type 1 Diabetes. In IEEE MultiConference on Systems and Control, pages 410-415, Buenos Aires, Argentina, 2016.

[114] Pau Herrero, Pantelis Georgiou, Christofer Toumazou, Benoît Delaunay, and Luc Jaulin. An efficient implementation of the sivia algorithm in a high-level numerical programming language. Reliable Computing, 16:239-251, 2012.

[115] Thomas Peyser, Eyal Dassau, Marc Breton, and Jay S. Skyler. The artificial pancreas: current status and future prospects in the management of diabetes. Annals of the New York Academy of Sciences, 1311(1):102-123, 2014.

[116] N. Rosales, F. Garelli, and H. De Battista. Automatic super-bolus and bolus-shaping features for insulin pumps. In 10th International Conference on Advanced Treatments E Technologies for Diabetes. Paris, Francia., 2017.

[117] Nicolás Rosales, Hernán De Battista, Josep Vehí, and Fabricio Garelli. Open-loop glucose control: Automatic IOB-based super-bolus feature for commercial insulin pumps. Computer Methods and Programs in Biomedicine, 159:145-158, 2018.

[118] Garelli F. Rosales N., De Battista H. Modelado y mitigación in-silico del efecto "rebote" durante el ejercicio en la diabetes tipo 1. In XVIII Reunión de trabajo en Procesamiento de la Información y Control (RPIC). UNS-CONICET. Bahia Blanca., 2019.

[119] N. Rosales, J. Vehí, F. Garelli, and De Battista H. Modelling and regulation of the effects of long-duration medium-intensity exercise in type 1 diabetic patients. In 12th International Conference on Advanced Technologies 83 Treatments for Diabetes. Berlin, Alemania., 2019.

[120] M E Willinska, L J Chassin, H C Schaller, L Schaupp, T R Pieber, and R Hovorka. Insulin kinetics in type 1 diabetes: Continuous and bolus delivery of rapid acting insulin. IEEE Transactions on Biomedical Engineering, 52(1):3-12, 2005. 
[121] G Nucci and C Cobelli. Models of subcutaneous insulin kinetics. A critical review. Computer Methods and Programs in Biomedicine, 62:249-257, 2000.

[122] B P Kovatchev, S Anderson, M Breton, S Patek, W Clarke, D Bruttomesso, A Maran, S Costa, A Avogaro, C Dalla Man, A Facchinetti, S Guerra, L Magni, D M Raimondo, G De Nicolao, E Renard, and C Cobelli. Personalized Subcutaneous Model-Predictive Closed-Loop Control of T1DM: Pilot Studies in the USA and Italy. Diabetes, 58(S1):A60, 2009.

[123] Androidaps documentación - https://androidaps.readthedocs.io/en/latest/en/.

[124] J Walsh, R Roberts, and L Heinemann. Confusion regarding duration of insulin action: A potential source for major insulin dose errors by bolus calculators. $J$. Diabetes Sci. Technol., 8(1):170-178, 2014.

[125] Diego de Pereda, Sergio Romero-Vivo, Beatriz Ricarte, Paolo Rossetti, Francisco Javier Ampudia-Blasco, and Jorge Bondia. Real-time estimation of plasma insulin concentration from continuous glucose monitor measurements. Computer Methods in Biomechanics and Biomedical Engineering, 19(9):934-942, 2015.

[126] Iman Hajizadeh, Mudassir Rashid, Kamuran Turksoy, Sediqeh Samadi, Jianyuan Feng, Nicole Frantz, Mert Sevil, Eda Cengiz, and Ali Cinar. Plasma Insulin Estimation in People with Type 1 Diabetes Mellitus. Industrial and Engineering Chemistry Research, 56(35):9846-9857, 2017.

[127] H Zisser, L Robinson, W Bevier, E Dassau, C Ellingsen, F J Doyle III, and L Jovanovic. Bolus calculator: a review of four "Smart" insulin pumps. Diabetes Technology and Therapeutics, 10(6):441-444, 2008.

[128] Gary Scheiner. Dealing with "d'oh". The Challenge, 2009.

[129] Mauro Boronat, Rosa M Sánchez Hernández, Julia Rodríguez-Cordero, Angelines Jiménez Ortega, and Francisco J Nóvoa. Suspension of basal insulin to avoid hypoglycemia in type 1 diabetes treated with insulin pump. Endocrinology, Diabetes 83 Metabolism Case Reports, 2015, 2015.

[130] A Revert, P Rossetti, R Calm, J Vehí, and J Bondia. Combining Basal-Bolus Insulin Infusion for Tight Postprandial Glucose Control: An in Silico Evaluation in Adults, Children, and Adolescents. J. Diabetes Sci. Technol, 4 (6):1424-1437, 2010.

[131] P Rossetti, F J Ampudia-Blasco, A Laguna, A Revert, J Vehí, J F Ascaso, and $\mathrm{J}$ Bondia. Evaluation of a novel continuous glucose monitoring-based method for mealtime insulin dosing the iBolus in subjects with type 1 diabetes using continuous subcutaneous insulin infusion therapy: a randomized controlled trial. Diabetes Technology and Therapeutics, 14(11):1043-1052, 2012.

[132] F Garelli, H De Battista, J Vehí, and F León-Vargas. Método y programa de ordenador para la determinación y distribución temporal de una dosis de insulina a un usuario, 2015.

[133] F Garelli, H De Battista, J Vehí, and F León-Vargas. Method and computer program for determination and time distribution of an insulin dose to a user, 2016.

[134] Graham C. Goodwin, Adrian M. Medioli, Diego S. Carrasco, Bruce R. King, and Yongji Fu. A fundamental control limitation for linear positive systems with application to Type 1 diabetes treatment. Automatica, 55:73-77, 2015. 
[135] Chiara Dalla Man, Michael Camilleri, and Claudio Cobelli. A system model of oral glucose absorption: Validation on gold standard data. IEEE Transactions on Biomedical Engineering, 53(12):2472-2478, 2006.

[136] David M Maahs, Bruce A Buckingham, Jessica R Castle, Ali Cinar, Edward R Damiano, Eyal Dassau, J Hans DeVries, Francis J Doyle, Steven C Griffen, Ahmad Haidar, Lutz Heinemann, Roman Hovorka, Timothy W Jones, Craig Kollman, Boris Kovatchev, Brian L Levy, Revital Nimri, David N O'Neal, Moshe Philip, Eric Renard, Steven J Russell, Stuart A Weinzimer, Howard Zisser, and John W Lum. Outcome Measures for Artificial Pancreas Clinical Trials: A Consensus Report: Table 1. Diabetes Care, 39(7):1175-1179, 2016.

[137] Dimitri Boiroux, Morten Hagdrup, Zeinab Mahmoudi, Niels Kjølstad Poulsen, Henrik Madsen, and John Bagterp Jørgensen. Model Identification using Continuous Glucose Monitoring Data for Type 1 Diabetes. IFAC-PapersOnLine, 49(7):759-764, 2016.

[138] Pau Herrero, Jorge Bondia, Oloruntoba Adewuyi, Peter Pesl, Mohamed ElSharkawy, Monika Reddy, Chris Toumazou, Nick Oliver, and Pantelis Georgiou. Enhancing automatic closed-loop glucose control in type 1 diabetes with an adaptive meal bolus calculator - in silico evaluation under intra-day variability. Computer Methods and Programs in Biomedicine, 146:125-131, 2017.

[139] Aleix Beneyto, Arthur Bertachi, Jorge Bondia, and Josep Vehi. A New Blood Glucose Control Scheme for Unannounced Exercise in Type 1 Diabetic Subjects. IEEE Transactions on Control Systems Technology, PP:1-8, 2018.

[140] Christian Ellingsen, Eyal Dassau, D Ph, Howard Zisser, Benyamin Grosman, Matthew W Percival, M Eng, Lois Jovanovič, and Francis J Doyle Iii. Safety Constraints in an Artificial Pancreatic. J. Diabetes Sci. Technol., 3(3):536-544, 2009.

[141] A Revert, F Garelli, J Pico, H De Battista, P Rossetti, J Vehí, and J Bondia. Safety auxiliary feedback element for the artificial pancreas in type 1 diabetes. IEEE Transactions on Biomedical Engineering, 2013.

[142] Arthur Bertachi, Aleix Beneyto, Charrise M. Ramkissoon, and Josep Vehí. Assessment of Mitigation Methods to Reduce the Risk of Hypoglycemia for Announced Exercise in a Uni-hormonal Artificial Pancreas. Diabetes Technology 83 Therapeutics, 20(4):285-295, 2018.

[143] Michael C Riddell, Ian W Gallen, Carmel E Smart, Craig E Taplin, Peter Adolfsson, Alistair N Lumb, Aaron Kowalski, Remi Rabasa-Lhoret, Rory J McCrimmon, Carin Hume, Francesca Annan, Paul A Fournier, Claudia Graham, Bruce Bode, Pietro Galassetti, Timothy W Jones, Iñigo San Millán, Tim Heise, Anne L Peters, Andreas Petz, and Lori M Laffel. Exercise management in type 1 diabetes: a consensus statement. The Lancet Diabetes \& Endocrinology, 5(5):377-390, 2017.

[144] Jacklyn A. Pivovarov, Craig E. Taplin, and Michael C. Riddell. Current perspectives on physical activity and exercise for youth with diabetes. Pediatric Diabetes, 16(4):242-255, 2015.

[145] Peter Adolfsson, Staffan Nilsson, Kerstin Albertsson-Wikland, and Bengt Lindblad. Hormonal response during physical exercise of different intensities in adolescents with type 1 diabetes and healthy controls. Pediatric Diabetes, 13(8):587-596, 2012. 
[146] Raul C Camacho, Pietro Galassetti, Stephen N Davis, and David H Wasserman. Glucoregulation during and after exercise in health and insulin-dependent diabetes. Exercise and sport sciences reviews, 33(1):17-23, 2005.

[147] Ronnie Aronson, Ruth E. Brown, Aihua Li, and Michael C. Riddell. Optimal insulin correction factor in post-high-intensity exercise hyperglycemia in adults with type 1 diabetes: The FIT study. Diabetes Care, 42(1):10-16, 2019.

[148] Sheri R. Colberg, Remmert Laan, Eyal Dassau, and David Kerr. Physical activity and type 1 diabetes: Time for a rewire? Journal of Diabetes Science and Technology, 9(3):609-618, 2015.

[149] M Derouich and A Boutayeb. The effect of physical exercise on the dynamics of glucose and insulin. Journal of biomechanics, 35(7):911-7, 2002.

[150] Chiara Dalla Man, Marc D Breton, and Claudio Cobelli. Physical Activity into the Meal Glucose-Insulin Model of Type 1 Diabetes: In silico Studies. Society, 3(1):5667, 2009.

[151] Michele Shciavon, Chiara Dalla Man, Yogish C. Kudva, Ananda Basu, and Claudio Cobelli. In Silico Optimization of Basal Insulin Infusion Rate during Exercise: Implication for Artificial Pancreas. J. Diabetes Sci. Technol., 44(4):361-381, 2013.

[152] Michele Schiavon, Ling Hinshaw, Ashwini Mallad, Chiara Dalla Man, Giovanni Sparacino, Matthew Johnson, Rickey Carter, Rita Basu, Yogish Kudva, Claudio Cobelli, and Ananda Basu. Postprandial glucose fluxes and insulin sensitivity during exercise: a study in healthy individuals. American journal of physiology. Endocrinology and metabolism, 305:E557-E566, 2013.

[153] Serafin Murillo. Diabetes tipo 1 y Deporte. EdikaMed, 2012.

[154] Emilia Fushimi, Nicolás Rosales, Hernán De Battista, and Fabricio Garelli. Artificial pancreas clinical trials: Moving towards closed-loop control using insulin-on-board constraints. Biomedical Signal Processing and Control, 2018.

[155] B P Kovatchev, E Renard, C Cobelli, H C Zisser, P Keith-Hynes, S M Anderson, S A Brown, D R Chernavvsky, M D Breton, L B Mize, A Farret, J Place, D Bruttomesso, S Del Favero, F Boscari, S Galasso, A Avogaro, L Magni, F Di Palma, C Toffanin, M Messori, E Dassau, and F J Doyle. Safety of Outpatient Closed-Loop Control: First Randomized Crossover Trials of a Wearable Artificial Pancreas. Diabetes Care, 37(7):1789-1796, 2014.

[156] Hood Thabit, Martin Tauschmann, Janet M Allen, Lalantha Leelarathna, Sara Hartnell, Malgorzata E Wilinska, Carlo L Acerini, Sibylle Dellweg, Carsten Benesch, Lutz Heinemann, Julia K Mader, Manuel Holzer, Harald Kojzar, Jane Exall, James Yong, Jennifer Pichierri, Katharine D Barnard, Craig Kollman, Peiyao Cheng, Peter C Hindmarsh, Fiona M Campbell, Sabine Arnolds, Thomas R Pieber, Mark L Evans, David B. Dunger, and Roman Hovorka. Home Use of an Artificial Beta Cell in Type 1 Diabetes. New England Journal of Medicine, 373(22):2129-2140, 2015.

[157] Stacey M Anderson, Dan Raghinaru, Jordan E Pinsker, Federico Boscari, Eric Renard, Bruce A Buckingham, Revital Nimri, Francis J Doyle, Sue A Brown, Patrick Keith-Hynes, Marc D. Breton, Daniel Chernavvsky, Wendy C. Bevier, Paige K. Bradley, Daniela Bruttomesso, Simone Del Favero, Roberta Calore, Claudio Cobelli, Angelo Avogaro, Anne Farret, Jerome Place, Trang T. Ly, Satya Shanmugham, 
Moshe Phillip, Eyal Dassau, Isuru S. Dasanayake, Craig Kollman, John W. Lum, Roy W. Beck, and Boris Kovatchev. Multinational home use of closed-loop control is safe and effective. Diabetes Care, 2016.

[158] R S Sánchez Peña, P Colmegna, L Grosembacher, M Breton, H De Battista, F Garelli, W Belloso, E Campos-Náñez, V Simonovich, V Beruto, P Scibona, and D Cherñavvsky. Artificial Pancreas: First Clinical Trials in Argentina. In 20th IFAC World Congress, pages 7997-8002, Toulouse, France, 2017.

[159] Patricio Colmegna, Fabricio Garelli, Hernán De Battista, and Ricardo Sánchez-Peña. Automatic regulatory control in type 1 diabetes without carbohydrate counting. Control Engineering Practice, 74:22-32, 2018.

[160] Kamuran Turksoy, Iman Hajizadeh, Sediqeh Samadi, Jianyuan Feng, Mert Sevil, Minsun Park, Laurie Quinn, Elizabeth Littlejohn, and Ali Cinar. Real-time insulin bolusing for unannounced meals with artificial pancreas. Control Engineering Practice, 59:159-164, 2017.

[161] F. Garelli, E. Fushimi, N. Rosales, and H. De Battista. Open to closed-loop transitions schemes for in vivo glucose control. In 10th International Conference on Advanced Treatments \& Technologies for Diabetes. Paris, Francia., 2017.

[162] Fabricio Garelli, Ricardo J. Mantz, and Hernán De Battista. Advanced Control for Constrained Processes and Systems. The Institution of Engineering and Technology, London, United Kingdom, 2011.

[163] F. J. Ampudia Blasco, I. Conget, J. Bondia, and J. Vehi. Improving postprandial glycaemia by a new developed closed-loop control system - closedloop4meals (cl4mcontrols). 2015.

[164] Vadim I Utkin. Sliding Modes in Control and Optimization. Springer Berlin Heidelberg, 1992.

[165] C C Palerm. Physiologic insulin delivery with insulin feedback: A control systems perspective. Computer Methods and Programs in Biomedicine, 102(2):130-137, 2011.

[166] G M Steil, K Rebrin, C Darwin, F Hariri, and M F Saad. Feasibility of automating insulin delivery for the treatment of type 1 diabetes. Diabetes, 55(12):3344-3350, 2006 .

[167] Stuart A Weinzimer, Garry M Steil, Karena L Swan, Jim Dziura, Natalie Kurtz, and William V Tamborlane. Fully Automated Closed-Loop Insulin Delivery Versus Semiautomated Hybrid Control in Pediatric Patients With Type 1 Diabetes Using an Artificial Pancreas. Diabetes Care, 31(5):934-939, 2008.

[168] Patricio H. Colmegna, Ricardo S. Sanchez-Pena, Ravi Gondhalekar, Eyal Dassau, and Frank J. Doyle. Switched LPV Glucose Control in Type 1 Diabetes. IEEE Transactions on Biomedical Engineering, 63(6):1192-1200, 2016.

[169] João P. Hespanha and A. Stephen Morse. Switching between stabilizing controllers. Automatica, 38(11):1905-1917, 2002.

[170] Eyal Dassau, B Wayne Bequette, Bruce A Buckingham, and Francis J Doyle. Detection of a Meal Using Continuous Glucose Monitoring. Diabetes Care, 31(2):295-300, 2008. 
[171] C S Hughes, S D Patek, M Breton, and B P Kovatchev. Anticipating the next meal using meal behavioral profiles: A hybrid model-based stochastic predictive control algorithm for T1DM. Comput. Methods Programs Biomed., 102(2):138-148, 2011.

[172] R A Harvey, E Dassau, H Zisser, D E Seborg, and F J Doyle I I I. Design of the glucose rate increase detector: A meal detection module for the health monitoring system. Journal of diabetes science and technology, 8(2):307-20XR: EMBASE 2014339886-20XR: EMBASE 20143398, 2014.

[173] K Turksoy, S Samadi, J Feng, E Littlejohn, L Quinn, and A Cinar. Meal Detection in Patients With Type 1 Diabetes: A New Module for the Multivariable Adaptive Artificial Pancreas Control System. IEEE J. Biomed. Health Inform., 20(1):47-54, 2016.

[174] S Samadi, K Turksoy, I Hajizadeh, J Feng, M Sevil, and Ali Cinar. Meal Detection and Carbohydrate Estimation Using Continuous Glucose Sensor Data. IEEE J. Biomed. Health Inform., 21(3):619-627, 2017.

[175] Emilia Fushimi, Patricio Colmegna, Hernán De Battista, Fabricio Garelli, and Ricardo Sánchez-Peña. Artificial pancreas: Evaluating the ARG algorithm without meal announcement. Journal of Diabetes Science and Technology, 13(6):1035-1043, 2019 .

[176] S D Patek, L Magni, E Dassau, C Hughes-Karvetski, C Toffanin, G De Nicolao, S Del Favero, M Breton, C Dalla Man, E Renard, H Zisser, F J Doyle III, C Cobelli, B P Kovatchev, and International Artificial Pancreas (iAP) Study Group. Modular Closed-Loop Control of Diabetes. IEEE Trans. Biomed. Eng, 59(11):2986-2999, 2012.

[177] P Keith-Hynes, B Mize, A Robert, and J Place. The Diabetes Assistant: A Smartphone-Based System for Real-Time Control of Blood Glucose. Electronics, 3(4):609-623, 2014.

[178] William Clarke and Boris Kovatchev. Statistical Tools to Analyze Continuous Glucose Monitor Data. Diabetes Technology and Therapeutics, 11(S1):S-45--S- 54 , 2009 .

[179] Chiara Fabris, Stephen D. Patek, and Marc D. Breton. Are Risk Indices Derived from CGM Interchangeable with SMBG-Based Indices? Journal of Diabetes Science and Technology, 10(1):50-59, 2016.

[180] Michael J Thompson, Laya Ekhlaspour, Laura A Young, Bruce A Buckingham, Kendra L Magyar, Hui Zheng, Steven J Russell, Samir Malkani, Paula Clinton, Debbie Mondesir, Rajendranath R Selagamsetty, Trang Ly, M Sue Kirkman, Manasi Sinha, Jamie Diner, Edward R Damiano, April Goley, Darrell M Wilson, Eliana Frank, J Paul Lock, Firas H El-Khatib, David M Harlan, Milana Dezube, John B Buse, Daniel DeSalvo, Courtney Balliro, Aryan Esmaeili, Celia Hartigan, Mallory A Hillard, and Lisa Norlander. Home use of a bihormonal bionic pancreas versus insulin pump therapy in adults with type 1 diabetes: a multicentre randomised crossover trial. The Lancet, 389(10067):369-380, 2016.

[181] Véronique Gingras, Ahmad Haidar, Virginie Messier, Laurent Legault, Martin Ladouceur, and Rémi Rabasa-Lhoret. A Simplified Semiquantitative Meal Bolus Strategy Combined with Single- and Dual-Hormone Closed-Loop Delivery in Patients with Type 1 Diabetes: A Pilot Study. Diabetes Technology and Therapeutics, 18(8):464-471, 2016. 
[182] N. Rosales, F. M. León Vargas, F. Garelli, and H. De Battista. Hypoglycemia prevention in closed-loop glycemic control via constraints in the glucose slope. In 11th International Conference on Advanced Technologies $\& 3$ Treatments for Diabetes. Viena, Austria., 2018.

[183] Jay S Skyler. The Economic Burden of Diabetes and the Benefits of Improved Glycemic Control: The Potential Role of a Continuous Glucose Monitoring System. Diabetes Technology and Therapeutics, 2(supplement 1):7-12, 2000.

[184] Thomas Danne, Revital Nimri, Tadej Battelino, Richard M. Bergenstal, Kelly L. Close, J. Hans DeVries, Satish Garg, Lutz Heinemann, Irl Hirsch, Stephanie A. Amiel, Roy Beck, Emanuele Bosi, Bruce Buckingham, Claudio Cobelli, Eyal Dassau, Francis J. Doyle, Simon Heller, Roman Hovorka, Weiping Jia, Tim Jones, Olga Kordonouri, Boris Kovatchev, Aaron Kowalski, Lori Laffel, David Maahs, Helen R. Murphy, Kirsten Nørgaard, Christopher G. Parkin, Eric Renard, Banshi Saboo, Mauro Scharf, William V. Tamborlane, Stuart A. Weinzimer, and Moshe Phillip. International consensus on use of continuous glucose monitoring. Diabetes Care, 40(12):1631-1640, 2017.

[185] Eyal Dassau, Fraser Cameron, Hyunjin Lee, B. Wayne Bequette, Howard Zisser, Lois Jovanovič, H. Peter Chase, Darrell M. Wilson, Bruce A. Buckingham, and Francis J. Doyle. Real-time hypoglycemia prediction suite using continuous glucose monitoring: A safety net for the artificial pancreas. Diabetes Care, 33(6):1249-1254, 2010.

[186] Mary B. Abraham, Martin de Bock, Nirubasini Paramalingam, Michael J. O'Grady, Trang T. Ly, Carly George, Anirban Roy, Glenn Spital, Sophy Karula, Kristine Heels, Rebecca Gebert, Jan M. Fairchild, Bruce R. King, Geoffrey R. Ambler, Fergus Cameron, Elizabeth A. Davis, and Timothy W. Jones. Prevention of Insulin-Induced Hypoglycemia in Type 1 Diabetes with Predictive Low Glucose Management System. Diabetes Technology $\&$ Therapeutics, 18(7):436-443, 2016.

[187] B. Wayne Bequette, Faye Cameron, Bruce A. Buckingham, David M. Maahs, and John Lum. Overnight Hypoglycemia and Hyperglycemia Mitigation for Individuals with Type 1 Diabetes: How Risks Can Be Reduced. IEEE Control Systems, 38(1):125-134, 2018.

[188] Bruce A Buckingham, Timothy S Bailey, Mark Christiansen, Satish Garg, Stuart Weinzimer, Bruce Bode, Stacey M Anderson, Ronald Brazg, Trang T Ly, and Francine R Kaufman. Evaluation of a Predictive Low-Glucose Management System In-Clinic. Diabetes Technology and Therapeutics, 19(5):288-292, 2017.

[189] Roy W. Beck, Dan Raghinaru, R. Paul Wadwa, H. Peter Chase, David M. Maahs, and Bruce A. Buckingham. Frequency of morning ketosis after overnight insulin suspension using an automated nocturnal predictive low glucose suspend system. Diabetes Care, 37(5):1224-1229, 2014.

[190] David M Maahs, Peter Calhoun, Bruce A Buckingham, H Peter Chase, Irene Hramiak, John Lum, Fraser Cameron, B Wayne Bequette, Tandy Aye, Terri Paul, Robert Slover, R Paul Wadwa, Darrell M Wilson, Craig Kollman, and Roy W Beck. A Randomized Trial of a Home System to Reduce Nocturnal Hypoglycemia in Type 1 Diabetes. Diabetes Care, 37(7):1885-1891, 2014.

[191] Alex Zhong, Pratik Choudhary, Chantal McMahon, Pratik Agrawal, John B Welsh, Toni L Cordero, and Francine R Kaufman. Effectiveness of Automated Insulin Management Features of the MiniMed(B640G Sensor-Augmented Insulin Pump. Diabetes Technology and Therapeutics, 18(10):657-663, 2016. 
[192] Pratik Choudhary, Birthe S. Olsen, Ignacio Conget, John B. Welsh, Linda Vorrink, and John J. Shin. Hypoglycemia Prevention and User Acceptance of an Insulin Pump System with Predictive Low Glucose Management. Diabetes Technology and Therapeutics, 18(5):288-291, 2016.

[193] Thomas Danne, Christiana Tsioli, Olga Kordonouri, Sarah Blaesig, Kerstin Remus, Anirban Roy, Barry Keenan, Scott W. Lee, and Francine R. Kaufman. The PILGRIM Study: In Silico Modeling of a Predictive Low Glucose Management System and Feasibility in Youth with Type 1 Diabetes During Exercise. Diabetes Technology and Therapeutics, 16(6):338-347, 2014.

[194] Tadej Battelino, Revital Nimri, Klemen Dovc, Moshe Phillip, and Natasa Bratina. Prevention of hypoglycemia with predictive low glucose insulin suspension in children with type 1 diabetes: A randomized controlled trial. Diabetes Care, 40(6):764-770, 2017.

[195] V. Gage, K. Caswell, F. Cameron, E. Dassau, H. P. Chase, P. Clinton, B. W. Bequette, F. J. Doyle, H. Lee, J. Wilkinson, E. Cobry, and B. Buckingham. Prevention of Nocturnal Hypoglycemia Using Predictive Alarm Algorithms and Insulin Pump Suspension. Diabetes Care, 33(5):1013-1017, 2010.

[196] Gregory P. Forlenza, Zoey Li, Bruce A. Buckingham, Jordan E. Pinsker, Eda Cengiz, R. Paul Wadwa, Laya Ekhlaspour, Mei Mei Church, Stuart A. Weinzimer, Emily Jost, Tatiana Marcal, Camille Andre, Lori Carria, Vance Swanson, John W. Lum, Craig Kollman, William Woodall, and Roy W. Beck. Predictive low-glucose suspend reduces hypoglycemia in adults, adolescents, and children with type 1 diabetes in an at-home randomized crossover study: Results of the PROLOG trial. Diabetes Care, 41(10):2155-2161, 2018.

[197] Vikash Dadlani, Jordan E. Pinsker, Eyal Dassau, and Yogish C. Kudva. Advances in Closed-Loop Insulin Delivery Systems in Patients with Type 1 Diabetes. Current Diabetes Reports, 18(10), 2018.

[198] Silvia Oviedo, Josep Vehí, Remei Calm, and Joaquim Armengol. A review of personalized blood glucose prediction strategies for T1DM patients. International Journal for Numerical Methods in Biomedical Engineering, 33(6):e2833, 2016.

[199] Jorge Bondia, Sergio Romero-Vivo, Beatriz Ricarte, and Jose Luis Diez. Insulin Estimation and Prediction: A Review of the Estimation and Prediction of Subcutaneous Insulin Pharmacokinetics in Closed-Loop Glucose Control. IEEE Control Systems, 38(1):47-66, 2018.

[200] Meriyan Eren-Oruklu, Ali Cinar, Lauretta Quinn, and Donald Smith. Adaptive control strategy for regulation of blood glucose levels in patients with type 1 diabetes. Journal of Process Control, 19(8):1333-1346, 2009.

[201] Carlo Novara, Ivana Rabbone, and Davide Tinti. Data-Driven Disturbance Estimation and Control With Application to Blood Glucose Regulation. IEEE Transactions on Control Systems Technology, PP:1-15, 2019.

[202] Kirthi S Walgama, D Grant Fisher, and Sirish L Shah. Control of processes with noise and time delays. AIChE Journal, 35(2):213-222, 1989.

[203] R E Kalman. A New Approach to Linear Filtering and Prediction Problems. Journal of Basic Engineering, 82(1):35, 1960. 
[204] F. Garelli, R. J. Mantz, and H. De Battista. Limiting interactions in decentralized control of MIMO systems. Journal of Process Control, 16(5):473-483, 2006.

[205] B. Wayne Bequette. Continuous glucose monitoring: Real-time algorithms for calibration, filtering, and alarms. Journal of Diabetes Science and Technology, 4(2):404418,2010 .

[206] P Soru, G De Nicolao, C Toffanin, C Dalla Man, C Cobelli, and L Magni. MPC based artificial pancreas: Strategies for individualization and meal compensation. Annual Reviews in Control, 36:118-128, 2012.

[207] Tadej Battelino, Thomas Danne, Richard M. Bergenstal, Stephanie A. Amiel, Roy Beck, Torben Biester, Emanuele Bosi, Bruce A. Buckingham, William T. Cefalu, Kelly L. Close, Claudio Cobelli, Eyal Dassau, J. Hans DeVries, Kim C. Donaghue, Klemen Dovc, Francis J. Doyle, Satish Garg, George Grunberger, Simon Heller, Lutz Heinemann, Irl B. Hirsch, Roman Hovorka, Weiping Jia, Olga Kordonouri, Boris Kovatchev, Aaron Kowalski, Lori Laffel, Brian Levine, Alexander Mayorov, Chantal Mathieu, Helen R. Murphy, Revital Nimri, Kirsten Nørgaard, Christopher G. Parkin, Eric Renard, David Rodbard, Banshi Saboo, Desmond Schatz, Keaton Stoner, Tatsuiko Urakami, Stuart A. Weinzimer, and Moshe Phillip. Clinical targets for continuous glucose monitoring data interpretation: Recommendations from the international consensus on time in range. Diabetes Care, 42(8):1593-1603, 2019.

[208] Dawei Shi, Eyal Dassau, and Francis J. Doyle. Adaptive Zone Model Predictive Control of Artificial Pancreas Based on Glucose- and Velocity-Dependent Control Penalties. IEEE Transactions on Biomedical Engineering, 66(4):1045-1054, 2019.

[209] Daniel Chernavvsky and Ricardo S. Sanchez Peña. Artificial Pancreas: Current Situation and Future Directions. Number March. Academic Press, 2019.

[210] Dana Lewis. History and Perspective on DIY Closed Looping. Journal of Diabetes Science and Technology, 13(4):790-793, 2019. 